\title{
Untersuchungen auf wasserlösliche Kohlenhydrate, Ertragsleistung und Inhaltsstoffe bei Futtergräsern zur Verbesserung der Verdaulichkeit
}

\author{
Dissertation \\ zur Erlangung des Doktorgrades \\ der Fakultät für Agrarwissenschaften \\ der Georg-August-Universität Göttingen
}

\author{
vorgelegt von \\ Walid Soufan \\ geboren in Syrien
}

Göttingen, Juli 2008 
D 7

1. Referent: Prof. Dr. Jörg Michael Greef

2. Referent: Prof. Dr. Johannes Isselstein

Tag der mündlichen Prüfung: 07. Juli 2008 


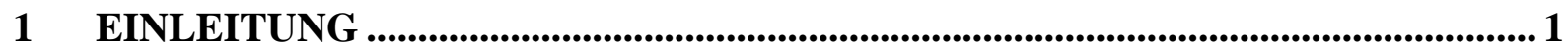

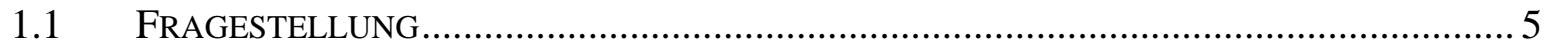

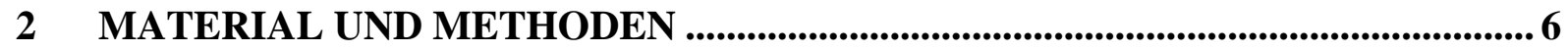

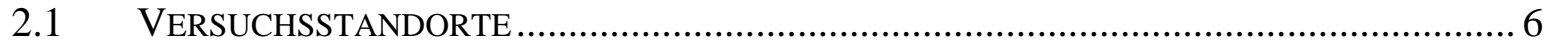

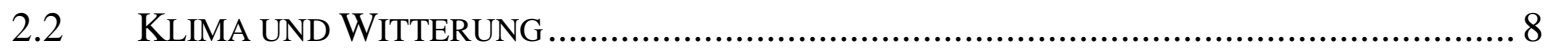

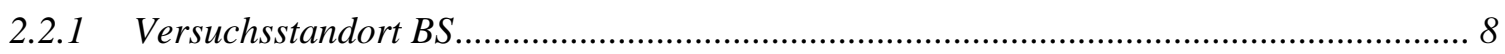

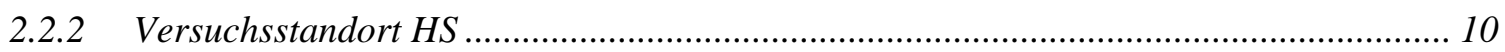

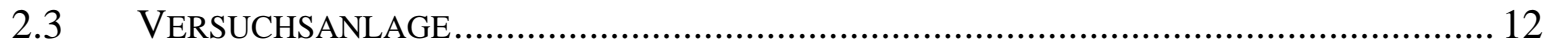

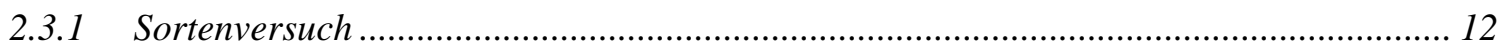

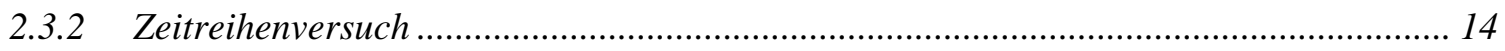

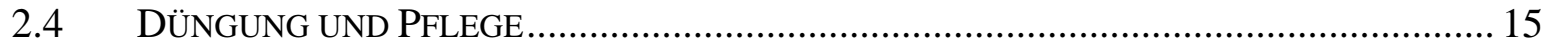

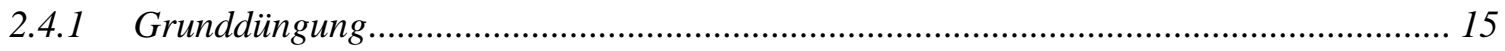

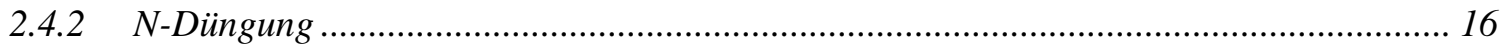

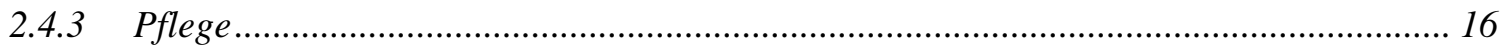

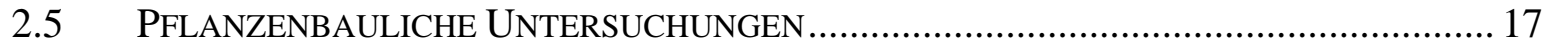

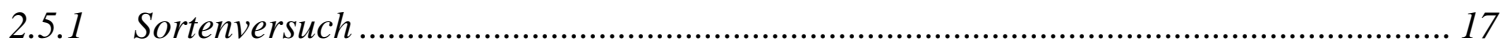

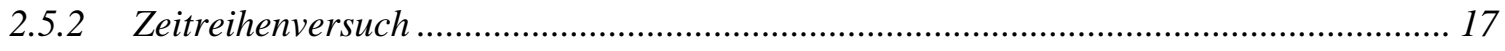

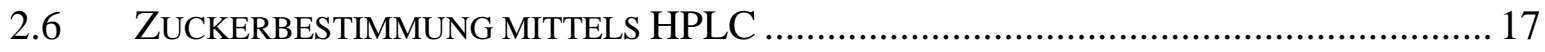

2.7 BestimMung DES PolyMERISATIONSGRAd VON FRUKTAN.................................... 17

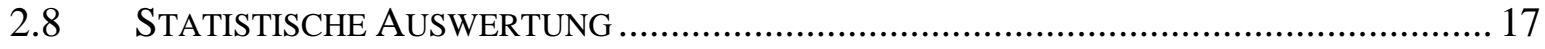

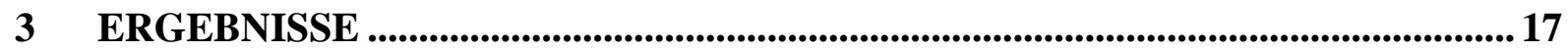

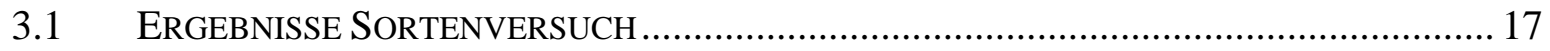

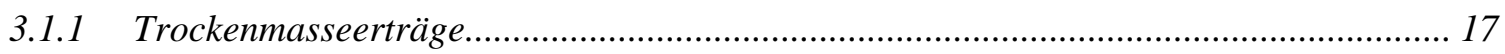

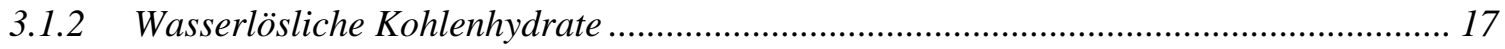

3.1.3 Verdaulichkeit (Enzymlösliche organischen Substanz) ................................................... 17

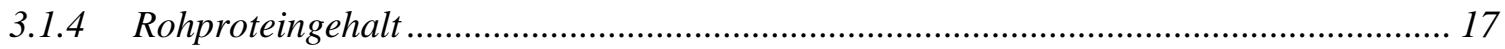

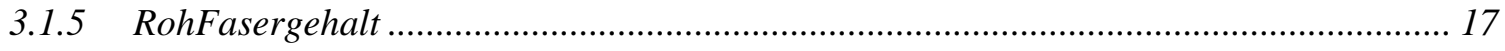

3.1.6 Die Korrelationen zwischen den wasserlöslichen Kohlenhydraten und dem TM-Ertrag, der Verdaulichkeit, dem Rohproteingehalt und dem Rohfasergehalt......................................... 17

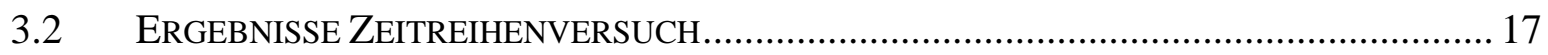

3.2.1 Wasserlösliche Kohlenhydrate und Fruktangehalt im Verlauf des ersten Aufwuchses... 17

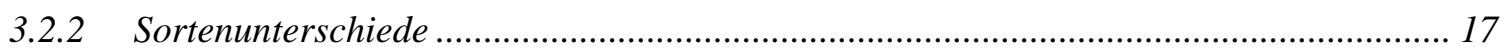

3.2.3 Wasserlösliche Kohlenhydrate und Fruktangehalt in den Grasfraktionen ...................... 17

3.2.4 Tageszeiteneinfluss auf WIK und Fruktangehalt ......................................................... 17 
3.2.5 Temperatureinfluss auf WIK und Fruktangehalt ........................................................... 17

3.2.6 N-Düngungseinfluss auf WIK und Fruktangehalt.......................................................... 17

3.2.7 Verteilung des Fruktangehaltes nach dem Polymerisationsgrad .................................... 17

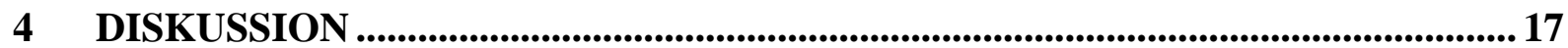

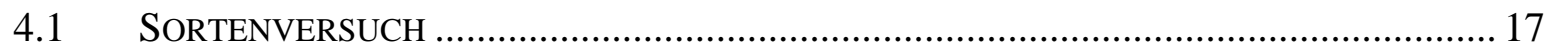

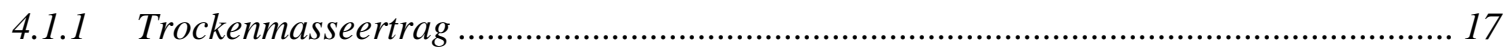

4.1.2 Wasserlösliche Kohlenhydrate ................................................................................ 17

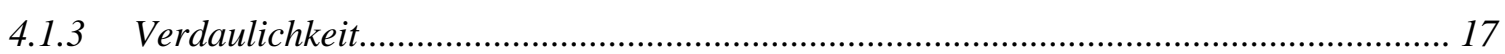

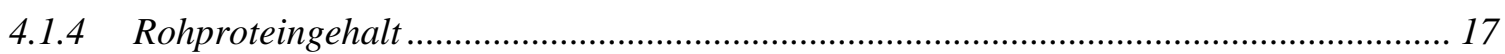

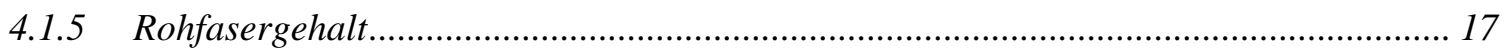

4.1.6 Die Korrelationen zwischen wasserlöslichen Kohlenhydraten und TM-Ertrag,

Verdaulichkeit, Rohproteingehalt und Rohfasergehalt. ............................................................. 17

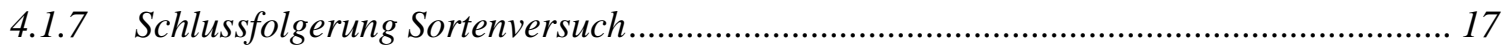

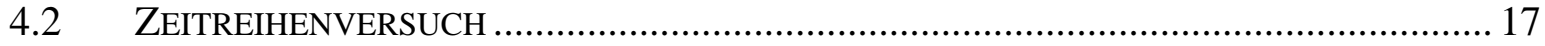

4.2.1 Wasserlösliche Kohlenhydrate und Fruktangehalt......................................................... 17

4.2.2 Verteilung der Fruktangehaltes nach dem Polymerisationsgrad ..................................... 17

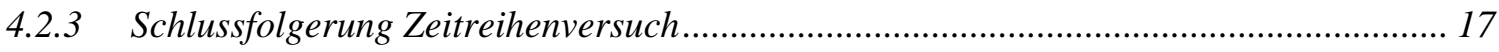

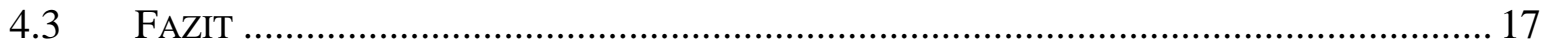

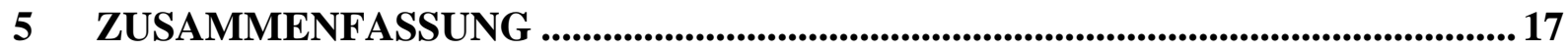

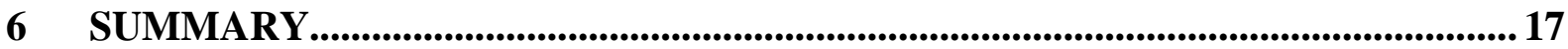

$7 \quad$ LITERATURVERZEICHNIS ....................................................................................... 17

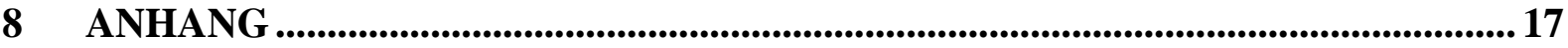




\section{Abbildungsverzeichnis}

Abb. 1: monatliche Niederschlagssummen [mm] des Anlagejahres 2005 und der Hauptversuchsjahre 2006 und 2007 sowie das langjährige Mittel (1962-2005) für den Versuchsstandort Braunschweig.

Abb. 2: Monatsmitteltemperaturen [ ${ }^{\circ} \mathrm{C}$ ] des Anlagejahres 2005 und der Hauptversuchsjahre 2006 und 2007 sowie das langjährige Mittel (1962-2005) für den Versuchsstandort Braunschweig. 9

Abb. 3: Monatliche Niederschlagssummen [mm] des Anlagejahres 2005 und der Hauptversuchsjahre 2006 und 2007 sowie das langjährige Mittel (2005-2007) für den Versuchsstandort Hof Steimke.

Abb. 4: Monatsmitteltemperaturen [ ${ }^{\circ} \mathrm{C}$ ] des Anlagejahres 2005 und der Hauptversuchsjahre 2006 und 2007 sowie das langjährige Mittel (2005-2007) für den Versuchsstandort Hof Steimke. 11

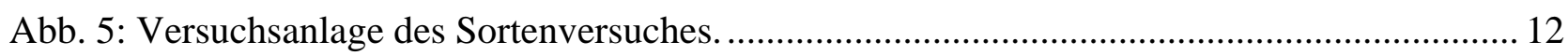

Abb. 6: Versuchsanlage des Zeitreihenversuches.................................................................... 15

Abb. 7: Chromatogramm eines Gemisches von Beneo GR und Beneo HP im Verhältnis 1:1....... 17 Abb. 8: TM-Ertrag dt/ha für fünf Schnitte der FSV des $1 \mathrm{HVJ}$ an Versuchsstandort (BS), signifikante Unterschiede gelten für Säulen mit unterschiedlichen Buchstaben $(\mathrm{P}<0,05)$, Fehlerbalken kennzeichnen die Standardabweichung.....

Abb. 9: TM-Ertrag dt/ha der für fünf Schnitte der SSV des $1 \mathrm{HVJ}$ an Versuchsstandort (BS), signifikante Unterschiede gelten für Säulen mit unterschiedlichen Buchstaben $(\mathrm{P}<0,05)$, Fehlerbalken kennzeichnen die Standardabweichung. 17

Abb. 10: TM-Ertrag dt/ha für vier Schnitte der FSV des 1HVJ an Versuchsstandort (HS), signifikante Unterschiede gelten für Säulen mit unterschiedlichen Buchstaben $(\mathrm{P}<0,05)$, Fehlerbalken kennzeichnen die Standardabweichung.....

Abb. 11: TM-Ertrag dt/ha für vier Schnitte der SSV des 1HVJ an Versuchsstandort (HS), signifikante Unterschiede gelten für Säulen mit unterschiedlichen Buchstaben $(\mathrm{P}<0,05)$, Fehlerbalken kennzeichnen die Standardabweichung.....

Abb. 12: TM-Ertrag dt/ha für fünf Schnitte der FSV des 2HVJ an Versuchsstandort (BS), signifikante Unterschiede gelten für Säulen mit unterschiedlichen Buchstaben $(\mathrm{P}<0,05)$, Fehlerbalken kennzeichnen die Standardabweichung

Abb. 13: TM-Ertrag dt/ha für fünf Schnitte der SSV des 2HVJ an Versuchsstandort (BS), signifikante Unterschiede gelten für Säulen mit unterschiedlichen Buchstaben $(\mathrm{P}<0,05)$, Fehlerbalken kennzeichnen die Standardabweichung..... 
Abb. 14: TM-Ertrag dt/ha für vier Schnitte der FSV des 2HVJ an Versuchsstandort (HS), signifikante Unterschiede gelten für Säulen mit unterschiedlichen Buchstaben $(\mathrm{P}<0,05)$, Fehlerbalken kennzeichnen die Standardabweichung.

Abb. 15: TM-Ertrag dt/ha für vier Schnitte der SSV des 2HVJ an Versuchsstandort (HS), signifikante Unterschiede gelten für Säulen mit unterschiedlichen Buchstaben $(\mathrm{P}<0,05)$, Fehlerbalken kennzeichnen die Standardabweichung.

Abb. 16: Wasserlösliche Kohlenhydrate (\% i.d. TM) in verschiedenen Gräserarten des Grünlandes. Mittelwert aus den beiden Versuchsstandorten BS und HS und den beiden Versuchsjahren 1HVJ und 2HVJ bei frühem und spätem Schnittzeitpunkt. 17

Abb.17: Wasserlösliche Kohlenhydrate (\% i.d. TM) in tetraploiden und diploiden Sorten des Deutschen Weidelgrases, Mittelwert aus den beiden Versuchsstandorten BS und HS und den beiden Versuchsjahren 1HVJ und 2HVJ im 1. Schnitt bei frühem und spätem Schnittzeitpunkt. 17

Abb. 18: Wasserlösliche Kohlenhydrate (\% i.d. TM) in tetraploiden und diploiden Sorten des Deutschen Weidelgrases, Mittelwert aus den beiden Versuchsstandorten BS und HS und den beiden Versuchsjahren 1HVJ und 2HVJ im Jahresmittel bei frühem und spätem Schnittzeitpunkt. 17

Abb. 19: Wasserlösliche Kohlenhydrate (\% i.d. TM) im Verlauf der Vegetationsperiode (Mittel aus allen 20 Sorten und den beiden Versuchsjahren 1HVJ und 2HVJ) an Versuchsstandort BS.

Abb. 20: Fruktangehalt (\% i. d. WlK) in verschiedenen Gräserarten des Grünlandes (Mittel aus den beiden Versuchsstandorten BS und HS und der FSV und der SSV) in den Versuchsjahren 1HVJ und 2HVJ. 17

Abb. 21: Fruktangehalt (\% i. d. WlK) im Verlauf der Vegetationsperiode (Mittel aus allen 20 Sorten und den beiden Versuchsjahren 1HVJ und 2HVJ) am Versuchsstandort BS..... 17

Abb. 22: Verdaulichkeit (\% i.d. TM) in verschiedenen Gräserarten des Grünlandes. Mittelwert aus den beiden Versuchsstandorten BS und HS und den beiden Versuchsjahren 1HVJ und 2HVJ bei frühem und spätem Schnittzeitpunkt. 17

Abb. 23: Verdaulichkeit (\% i.d. TM) in tetraploiden und diploiden Sorten des Deutschen Weidelgrases, Mittelwert aus den beiden Versuchsstandorten BS und HS und den beiden Versuchsjahren 1HVJ und 2HVJ im 1. Schnitt bei frühem und spätem Schnittzeitpunkt....... 17 Abb. 24: Verdaulichkeit (\% i.d. TM) in tetraploiden und diploiden Sorten des Deutschen Weidelgrases, Mittelwert aus den beiden Versuchsstandorten BS und HS und den beiden Versuchsjahren 1HVJ und 2HVJ im Jahresmittel bei frühem und spätem Schnittzeitpunkt... 17 
Abb. 25: Die Verdaulichkeit (\% i.d. TM) im Verlauf der Vegetationsperiode (Mittel aus allen 20 Sorten und den beiden Versuchsjahren 1HVJ und 2HVJ) an Versuchsstandort BS

Abb. 26: Rohproteingehalt (\% i.d. TM) in verschiedenen Gräserarten des Grünlandes. Mittelwert aus den beiden Versuchsstandorten BS und HS und den beiden Versuchsjahren 1HVJ und 2HVJ bei frühem und spätem Schnittzeitpunkt.

Abb. 27: Rohproteingehalt (\% i.d. TM) von Deutschen Weidelgrassorten der Reifegruppe früh, mittel und spät, Mittelwert aus den beiden Versuchsstandorten BS und HS und den beiden Versuchsjahren 1HVJ und 2HVJ im 1. Schnitt bei frühem und spätem Schnittzeitpunkt....... 17 Abb. 28: Rohproteingehalt (\% i.d. TM) in tetraploiden und diploiden Sorten des Deutschen Weidelgrases, Mittelwert aus den beiden Versuchsstandorten BS und HS und den beiden Versuchsjahren 1HVJ und 2HVJ im 1. Schnitt bei frühem und spätem Schnittzeitpunkt....... 17 Abb. 29: Rohproteingehalt (\% i.d. TM) in tetraploiden und diploiden Sorten des Deutschen Weidelgrases, Mittelwert aus den beiden Versuchsstandorten BS und HS und den beiden Versuchsjahren 1HVJ und 2HVJ im Jahresmittel bei frühem und spätem Schnittzeitpunkt... 17 Abb. 30: Rohproteingehalt (\% i.d. TM) im Verlauf der Vegetationsperiode (Mittel aus allen 20 Sorten und den beiden Versuchsjahren 1HVJ und 2HVJ) an Versuchsstandort BS. 17

Abb. 31: Rohfasergehalt (\% i.d. TM) in verschiedenen Gräserarten des Grünlandes. Mittelwert aus den beiden Versuchsstandorten BS und HS und den beiden Versuchsjahren 1HVJ und 2HVJ bei frühem und spätem Schnittzeitpunkt.

Abb. 32: Rohfasergehalt (\% i.d. TM) von Deutschen Weidelgrassorten der Reifegruppe früh, mittel und spät, Mittelwert aus den beiden Versuchsstandorten BS und HS und den beiden Versuchsjahren 1HVJ und 2HVJ im 1. Schnitt bei frühem und spätem Schnittzeitpunkt....... 17 Abb. 33: Rohfasergehalt (\% i.d. TM) in tetraploiden und diploiden Sorten des Deutschen Weidelgrases, Mittelwert aus den beiden Versuchsstandorten BS und HS und den beiden Versuchsjahren 1HVJ und 2HVJ im 1. Schnitt bei frühem und spätem Schnittzeitpunkt....... 17 Abb. 34: Rohfasergehalt (\% i.d. TM) in tetraploiden und diploiden Sorten des Deutschen Weidelgrases, Mittelwert aus den beiden Versuchsstandorten BS und HS und den beiden Versuchsjahren 1HVJ und 2HVJ im Jahresmittel bei frühem und spätem Schnittzeitpunkt... 17 Abb. 35: Rohfasergehalt (\% i.d. TM) im Verlauf der Vegetationsperiode (Mittel aus allen 20 Sorten und den beiden Versuchsjahren 1HVJ und 2HVJ) an Versuchsstandort BS..... 17 Abb. 36: Wasserlösliche Kohlenhydrate (WlK) in Korrelation zu dem TM-Ertrag im Gras.......... 17 Abb. 37: Wasserlösliche Kohlenhydrate (WlK) in Korrelation zu der Verdaulichkeit (ELOS) im Gras. 
Abb. 38: Wasserlösliche Kohlenhydrate (WlK) in Korrelation zu dem Rohproteingehalt (RP) im Gras.

Abb. 39: Wasserlösliche Kohlenhydrate (WlK) in Korrelation zu dem Rohfasergehalt (RF) im Gras.

Abb. 40: Wasserlösliche Kohlenhydrate und Fruktangehalt der Gemischprobe im Verlauf des ersten Aufwuchses für alle Sorten im 2HVJ.

Abb. 41: Wasserlösliche Kohlenhydrate und Fruktangehalt des Stängels im Verlauf des ersten Aufwuchses für alle Sorten im 2HVJ.

Abb. 42: Wasserlösliche Kohlenhydrate und Fruktangehalt der Blätter im Verlauf des ersten Aufwuchses für alle Sorten im 2HVJ.

Abb. 43: Wasserlösliche Kohlenhydrate und Fruktangehalt in verschiedenen Deutschem Weidelgrassorten im Verflauf des ersten Aufwuchses, im Mittel der allen Ernten und der beiden Versuchsjahren (1HVJ und 2HVJ).

Abb. 44: Wasserlösliche Kohlenhydrate, Fruktangehalt und Fruktangehalt (\%) in den WlK in der Grasfraktion, im Sortenmittel Mittelwert aus der 10. und 11. Ernte im 2HVJ. 17

Abb. 45: Wasserlösliche Kohlenhydrate (WlK) in Korrelation zur Temperatur $\left({ }^{\circ} \mathrm{C}\right)$ im Mittel der Sorten und der beiden Versuchsjahre (1HVJ und 2HVJ). 17

Abb. 46: Fruktangehalt in Korrelation zur Temperatur $\left({ }^{\circ} \mathrm{C}\right)$ im Mittel der Sorten und der beiden Versuchsjahre (1HVJ und 2HVJ)......

Abb. 47: Verteilung des Fruktangehaltes (\%) nach dem Polymerisationsgrad für die Sorte Respect in der Gemischprobe im 2HVJ (2007) in allen Ernteterminen. 17

Abb. 48: Verteilung des Fruktangehaltes (\%) nach dem Polymerisationsgrad für die Sorte AberAvon in der Gemischprobe im 2HVJ (2007) in allen Ernteterminen. 17

Abb. 49: Verteilung des Fruktangehaltes (\%) nach dem Polymerisationsgrad für die Sorte Respect in den Blättern im 2HVJ (2007) in allen Erntenterminen. 17

Abb. 50: Verteilung des Fruktangehaltes (\%) nach dem Polymerisationsgrad für die Sorte AberAvon in den Blättern im 2HVJ (2007) in allen Ernteterminen. .... 17

Abb. 51: Verteilung des Fruktangehaltes (\%) nach dem Polymerisationsgrad für die Sorte Respect in den Stängeln im 2HVJ (2007) in allen Ernteterminen. 17

Abb. 52: Verteilung des Fruktangehaltes (\%) nach dem Polymerisationsgrad für die Sorte AberAvon in den Stängeln im 2HVJ (2007) in allen Ernteterminen. 


\section{Tabellenverzeichnis}

Tab. 1: Effekt eines erhöhten Zuckergehaltes auf die Milchproduktion. 4

Tab. 2: Standortbeschreibung des Sortenversuches an den zwei Versuchstandorten (BS und HS) und des Zeitreihenversuches. 7

Tab. 3: pH-Wert, Phosphat- $\left(\mathrm{P}_{2} \mathrm{O}_{5}\right)-$, Kaliumoxid- $\left(\mathrm{K}_{2} \mathrm{O}\right)$ - und Magnesiumgehalte (Mg) im lufttrockenen Boden (Probenahme im Februar).

Tab. 4: Die Jahresdurchschnittstemperatur und Jahresniederschlag [mm] des Anlagejahres 2005 und der Hauptversuchsjahre 2006 und 2007 für die Versuchsstandorte BS und HS sowie das langjährige Mittel (1962-2005) für Versuchsstandort BS und (2005-2007) für Versuchsstandort HS.

Tab. 5: Verwendete Futtergräsersorten für den Sortenversuch (Züchter, Lateinischer Name, Deutscher Name, Ploidiegrugge, wasserlösliche kohlenhydrategehalt (Wlk-Ghelat) und Reifegruppe). 13

Tab. 6: Schnitttermine im Sortenversuch und allen Versuchsstandorten BS und HS 14

Tab. 7: NIRS Kalibrationen, erstellt aus dem Probenmaterial der Erntejahre 1994 bis 2001 für RP, RF, WlK, EULOS und RA und der Erntejahre 2002 bis 2005 für Fruktan.

Tab. 8: Erntetermine im Zeitreiheversuch in beiden Versuchsjahren 2006 und 2007.

Tab. 9: Versuchsjahresvergleich (2006/2007) für TM-Ertrag dt/ha Mittelwert aus allen Sorten im 1. Schnitt und im Jahresertrag.

Tab. 10: Schnittzeitpunktvergleich (FSV und SSV) für TM-Ertrag (dt/ha) Mittelwert aus allen Sorten und Versuchsjahren (2006 und 2007) im 1. Schnitt und im Jahresertrag.... 17

Tab. 11: TM-Erträge (dt/ha) an beiden Versuchsstandorten (BS und HS) im 1. Schnitt. Mittelwert aus allen Sorten.

Tab. 12: TM-Ertrag (dt/ha) in Abhängigkeit von den Sortenreifegruppen (früh, mittel und spät) von Deutschen Weidelgras am Versuchsstandort BS im 1HVJ (2006) und im 2HVJ (2007).

Tab. 13: TM-Ertrag (dt/ha) in Abhängigkeit von den Sortenreifegruppen (früh, mittel und spät) von Deutschen Weidelgras am Versuchsstandort HS im 1HVJ (2006) und im 2HVJ (2007)

Tab. 14: TM-Ertrag (dt/ha) in Abhängigkeit von den Sortenreifengruppen (früh, mittel und spät) der Deutsches Weidelgras, im Mittel der beiden Versuchsjahren (1HVJ und 2HVJ) und der beiden Versuchsstandorten (BS und HS), im Jahresmittel. 
Tab. 15: Versuchsjahresvergleich (2006/2007) für wasserlösliche Kohlenhydrate (\% i.d. TM) Mittelwert aus allen Sorten im 1. Schnitt und im Jahresmittel. 17

Tab. 16: Schnittzeitpunktvergleich (FSV/SSV) für wasserlösliche Kohlenhydrate (\% i.d. TM) Mittelwert aus allen Sorten im 1. Schnitt und im Jahresmittel. 17

Tab. 17: Wasserlösliche Kohlenhydrate (\% i.d. TM) an beiden Versuchsstandorte (BS und HS) im 1. Schnitt. 17

Tab. 18: Wasserlösliche Kohlenhydrate (\% i.d. TM) in Abhängigkeit von den Sortenreifengruppen (früh, mittel und spät) der Deutsches Weidelgras am Versuchsstandort BS im 1HVJ (2006) und im 2HVJ (2007)........................................ 17

Tab. 19: Wasserlösliche Kohlenhydrate (\% i.d. TM) in Abhängigkeit von den Sortenreifengruppen (früh, mittel und spät) der Deutsches Weidelgras am Versuchsstandort HS im 1HVJ (2006) und im 2HVJ (2007). 17

Tab. 20: Wasserlösliche Kohlenhydrate (WlK) (\% i.d. TM) in Abhängigkeit von den Sortenreifengruppen (früh, mittel und spät) der Deutsches Weidelgras, im Mittel der beiden Versuchsjahren (1HVJ und 2HVJ) und der beiden Versuchsstandorten (BS und HS), im Jahresmittel.

Tab. 21: Versuchsjahresvergleich (2006/2007) für Enzymlösliche organischer Substanz (\% i.d. TM). Mittelwert aus allen Sorten im 1. Schnitt und im Jahresertrag. 17

Tab. 22: Schnittzeitpunktvergleich (FSV/SSV) für Enzymlösliche organischer Substanz (\% i.d. TM). Mittelwert aus allen Sorten im 1. Schnitt und im Jahresmittel.

Tab. 23: Enzymlösliche organischer Substanz (\% i.d. TM) an beiden Versuchsstandorte (BS und HS) im 1. Schnitt.

Tab. 24: Enzymlösliche organischer Substanz (\% i.d. TM) in Abhängigkeit von den Sortenreifengruppen (früh, mittel und spät) der Deutsches Weidelgras am Versuchsstandort BS im 1HVJ (2006) und im 2HVJ (2007). 17

Tab. 25: Enzymlösliche organischer Substanz (\% i.d. TM) in Abhängigkeit von den Sortenreifengruppen (früh, mittel und spät) der Deutsches Weidelgras am Versuchsstandort HS im 1HVJ (2006) und im 2HVJ (2007). 17

Tab. 26: Enzymlösliche organischer Substanz (ELOS) (\% i.d. TM) in Abhängigkeit von den Sortenreifengruppen (früh, mittel und spät) der Deutsches Weidelgras, im Mittel der beiden Versuchsjahren (1HVJ und 2HVJ) und der beiden Versuchsstandorten (BS und HS), im Jahresmittel. 17

Tab. 27: Versuchsjahresvergleich (2006/2007) für Rohproteingehalt (\% i.d. TM). Mittelwert aus allen Sorten im 1 . Schnitt und im Jahresertrag. 
Tab. 28: Schnittzeitpunktvergleich (FSV/SSV) für Rohproteingehalt (\% i.d. TM). Mittelwert aus allen Sorten im 1. Schnitt und im Jahresmittel.

Tab. 29: Rohproteingehalt (\% i.d. TM) an beiden Versuchsstandorte (BS und HS) im 1. Schnitt.

Tab. 30: Versuchsjahresvergleich (2006/2007) für Rohfasergehalt (\% i.d. TM). Mittelwert aus allen Sorten im 1. Schnitt und im Jahresertrag.

Tab. 31: Schnittzeitpunktvergleich (FSV/SSV) für Rohfasergehalt (\% i.d. TM). Mittelwert aus allen Sorten im 1. Schnitt und im Jahresmittel.

Tab. 32: Rohfasergehalt (\% i.d. TM) an beiden Versuchsstandorte (BS und HS) im 1. Schnitt.

Tab. 33: Wasserlösliche Kohlenhydrate (WlK) \% i.d. TM und Fruktangehalt \% i.d. TM im Verlauf des 1. Aufwuchses des 1HVJ und des 2HVJ über alle fünf Sorten und im Mittel aller Ernten bei demselben phänologischen Stadium.

Tab.34: Die Phänologischen Stadien (BBCH) der Deutschen Weidelgrassorten zu den Erntenterminen des 1HVJ und 2. HVJ des Zeitreihenversuches.

Tab. 35: Wasserlösliche Kohlenhydrate (WlK) \% i.d. TM und Fruktangehalt \% i.d. TM im Verlauf des 1. Aufwuchses des 1HVJ in Abhängigkeit von Tageszeiten (8 Uhr und 13 Uhr) im Mittel der fünf Sorten, aller Ernten und der beiden N-Düngungsstufen (normal und niedrig).

Tab. 36: Wasserlösliche Kohlenhydrate (WlK) \% i.d. TM und Fruktangehalt \% i.d. TM im Verlauf des 1. Aufwuchses des 1HVJ in Abhängigkeit von N-Düngungsstufen (normal und niedrig) im Mittel der fünf Sorten, aller Ernten und der beiden Tageszeiten (8 Uhr und $13 \mathrm{Uhr})$.

Tab. A1: Der prozentuale Anteil des 1. Schnittes am Jahres-TM-Ertrag auf beiden Versuchsstandorten (BS und HS), bei beiden Schnittzeitpunktsvarianten (FSV und SSV) und im beiden Hauptsversuchsjahren (2006 und 2007).

Tab. A2: Verteilung des Fruktangehaltes (\%) nach dem Polymerisationsgrad für Sorte Respect in Gemischprobe, Stängel und Blätter im 2HVJ (2007) in allen Erntenterminen.

Tab. A3: Verteilung des Fruktangehaltes (\%) nach dem Polymerisationsgrad für Sorte Licarta in Gemischprobe, Stängel und Blätter im 2HVJ (2007) in allen Erntenterminen. 17

Tab. A4: Verteilung des Fruktangehaltes (\%) nach dem Polymerisationsgrad für Sorte EGB1 in Gemischprobe, Stängel und Blätter im 2HVJ (2007) in allen Erntenterminen. 
Tab. A5: Verteilung des Fruktangehaltes (\%) nach dem Polymerisationsgrad für Sorte Rodrigo in Gemischprobe, Stängel und Blätter im 2HVJ (2007) in allen Erntenterminen. 17

Tab. A6: Verteilung des Fruktangehaltes (\%) nach dem Polymerisationsgrad für Sorte AberAvon in Gemischprobe, Stängel und Blätter im 2HVJ (2007) in allen

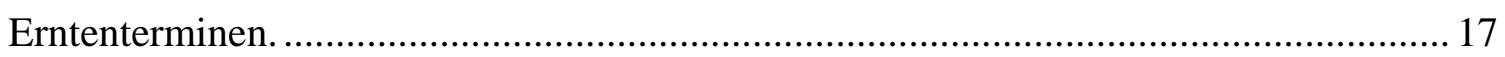




\section{Abkürzungsverzeichnis}

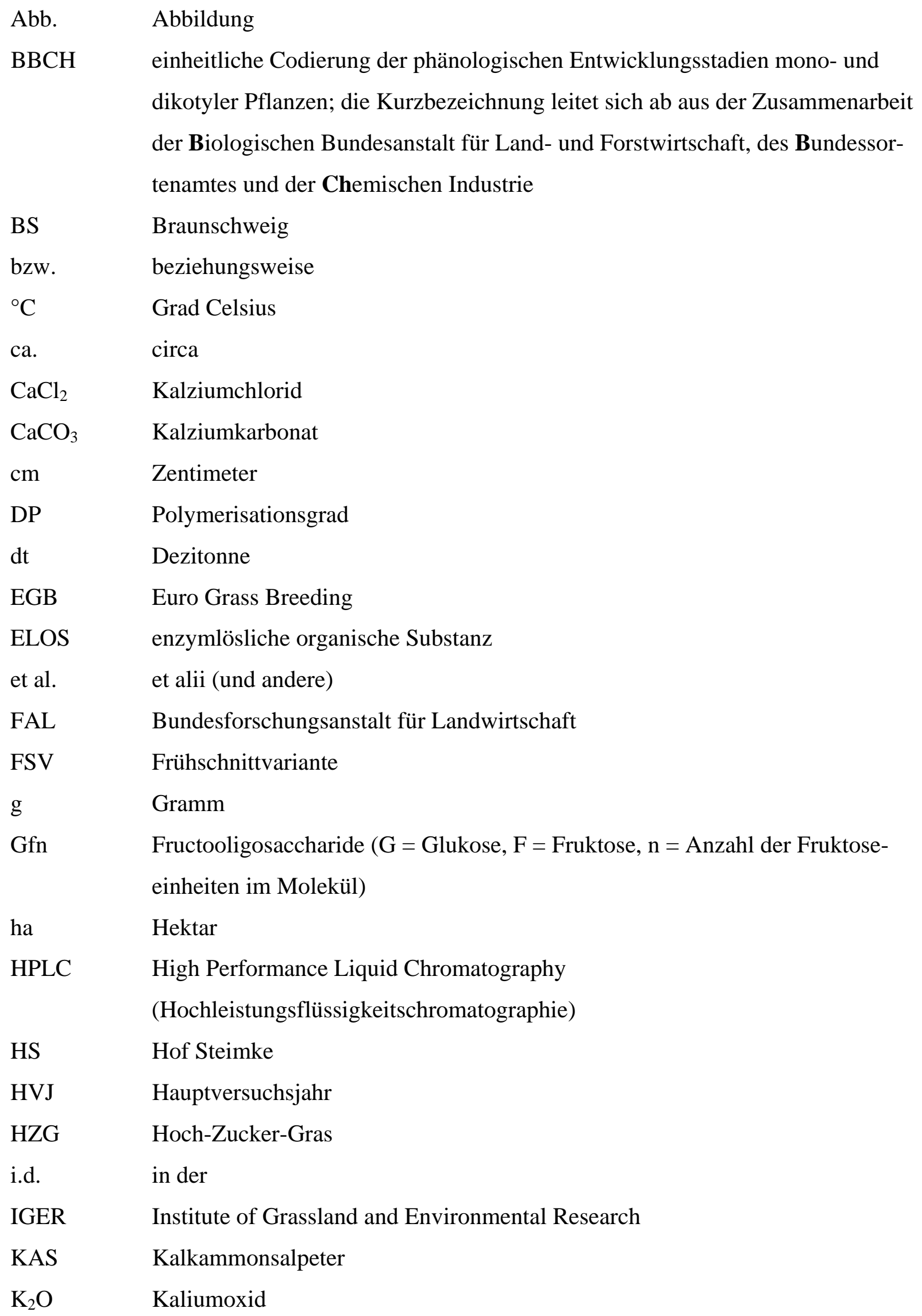




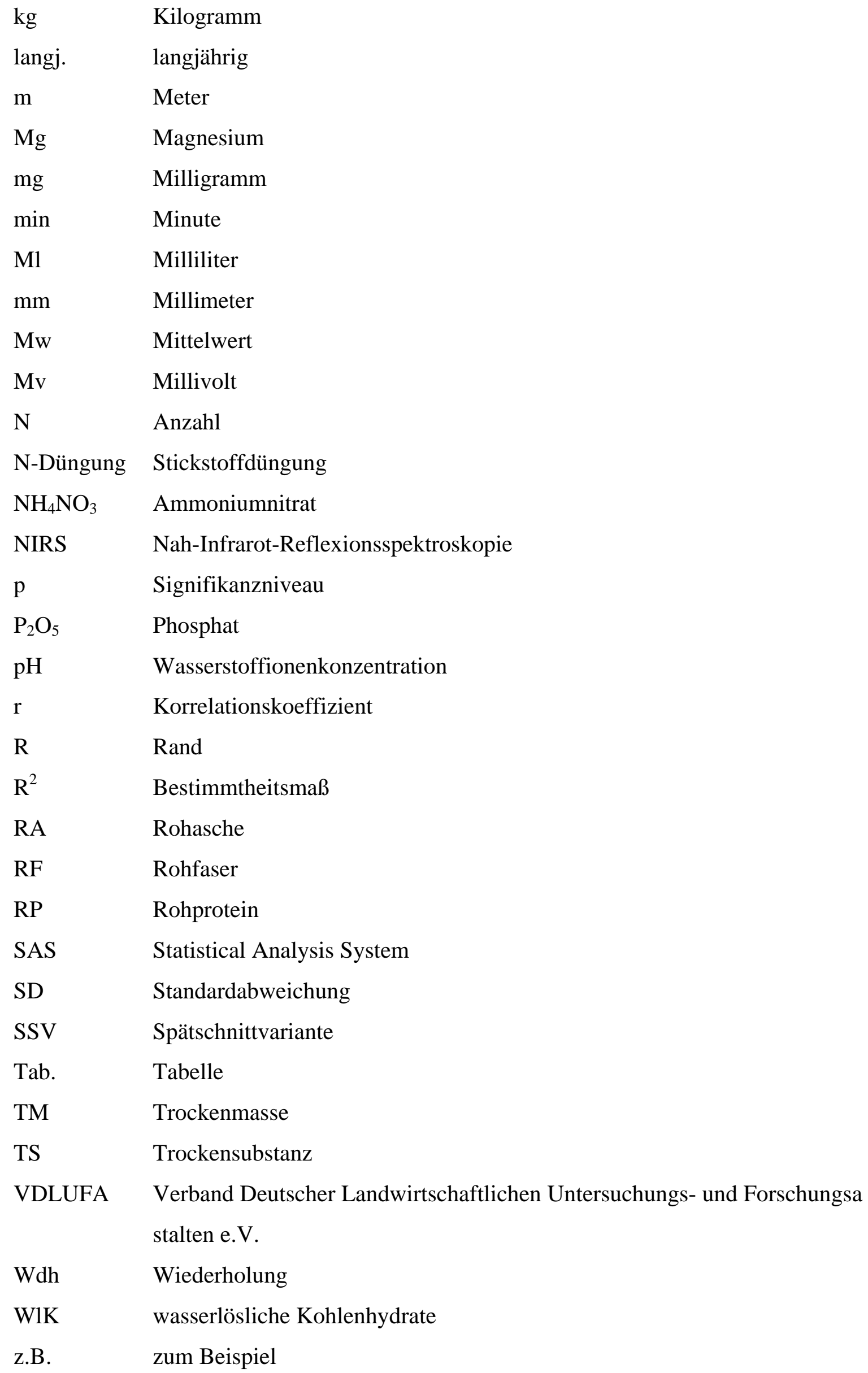

VDLUFA Verband Deutscher Landwirtschaftlichen Untersuchungs- und Forschungsa stalten e.V.

Wdh Wiederholung

WlK wasserlösliche Kohlenhydrate

z.B. zum Beispiel 


\section{$1 \quad$ Einleitung}

Eine wirtschaftliche und langfristig erfolgreiche Milchproduktion setzt die bedarfsgerechte Fütterung der Kühe voraus (Daccord, 1992). Zur Abdeckung des Nährstoffbedarfes bei sehr hohen Milchleistungen sind ein hoher Kraftfutteranteil und beste Grundfutterqualität die Voraussetzung (Gruber et al., 1995). Hertwig (2004) erwähnte, dass bei dem erreichten hohen Leistungsniveau in der Milchproduktion sich immer deutlicher zeigt: ohne ein energiereiches und hochverdauliches Grundfutter sind weitere Leistungssteigerungen nur sehr schwer zu erzielen. Bei einer niedrigen Grundfutterqualität war in der Hochlaktation ein wesentlich höherer Kraftfutteranteil zur Energieergänzung erforderlich. Neben der Milchmenge wurden auch die Inhaltsstoffe Fett und Protein sowohl durch eine höhere Grundfutterqualität als auch Kraftfuttermenge gesteigert (Gruber et al., 1995). Dabei ist jedoch zu berücksichtigen, dass ein hoher Kraftfutteranteil zu: 1- Grundfutterverdrängung durch Einfluss des Kraftfutters auf den PH-Wert und Essigsäure/Propionsäure- Verhältnis in Vormägen und 2- negative Wechselwirkungen zwischen Grund- und Kraftfutter auf Verdauungs- und Stoffwechselebene führt (Gruber et al., 1995). Auf Grund dieser Wechselwirkungen liegt die tatsächliche Energieversorgung bei Hochleistungskühen um 7 - 10 \% niedriger als die theoretisch (d.h. additiv) errechnete (Vermorel, 1989). Mit höherem Kraftfuttereinsatz erhöhen sich die Futterkosten, die in der Milchproduktion je nach Bestandesgröße 18 - 28 \% der gesamten Produktionskosten ausmachen (Pfingstner, 1993).

Nach Gruber et al. (1995) war die Milchleistung der Kühe bei hoher, im Vergleich zu niedriger Grundfutterqualität trotz jeweils bedarfsgerechter Kraftfutterergänzung, um etwa 1.000 kg höher. Daher ist davon auszugehen, dass eine hohe Grundfutterqualität die Voraussetzung für eine hohe Milchleistung ist. Hierbei spielen die Verdaulichkeit des Grundfutters, die Rohfaserfraktionen, der Zucker- und Proteingehalt eine entscheidende Rolle (Kalzendorf, 2008). Die Verdaulichkeit des Futters schließlich richtet sich nach den Gehalten und der Zusammensetzung der drei zuvor genannten Inhaltsstoffe Rohfaser, Zucker und Protein.

Nach Junk und Fisch (2006) sind die Zielgrößen der Grundfutterproduktion: an den Tierbestand angepasste gute Futterflächen, Qualitätserträge vom Grünland für Milchvieh, ertragsangepasste Düngung mit organischen Düngern und Mineraldüngern, dichte Grünlandnarben mit hohem Anteil an wertvollen Gräsern sowie die Erhaltung und Verbesserung des Grünlands durch Nach- und Übersaaten. 
Qualität und Energiegehalt des wirtschaftseigenen Grundfutters sind in hohem Maße vom Erntezeitpunkt, Witterung bei der Ernte, Ernteverfahren und Silierverlauf abhängig (Beimler und Eickmeyer, 2005).

Neben den Sorteneigenschaften wie Trockenmasseertrag, Mooreignung, Winterhärte, Ausdauer, Narbendichte, Erstschnitt- oder Nachwuchsbetonung, Krankheitsresistenzen, Reifezeitpunkt usw. werden in den Regionalempfehlungen die Futtermischungen vor allem vom Futterwert und dessen Parametern wie Verdaulichkeit, Zuckergehalt und Proteingehalt beeinflusst. (Eickmeyer, 2004). Diese Futterwertparameter einer Sorte sind für die Milch- und Fleischproduktion entscheidend.

Wegen der spezifischen Ansprüche an das Klima ist der Anbau von energiereichen Futterpflanzen wie z.B Mais in Nordeuropa erschwert. Daher ist es für eine leistungsstarke und ökonomische Rinderproduktion unumgänglich, nach alternativem Grundfutter mit hoher Energiedichte zu suchen (Martens und Greef, 2003). In dem Institute of Grassland and Environmental Research (IGER) in Großbritannien Pflanzenzuchtprogramm, an dem bereits seit 1980 an der Entwicklung von leistungsfähigen Sorten mit einem höheren Gehalt an Zucker gearbeitet wird, wurden Deutsche Weidelgrassorten (Lolium perenne) selektiert, die einen vergleichsweise hohen Gehalt an wasserlöslichen Kohlenhydraten besitzen.

Durch die Neuzüchtungen von Deutschem Weidelgras mit einem erhöhten Zuckergehalt, die in der Fütterung hochleistender Wiederkäuer sehr positiv zu bewerten sind, kann in gut geführten Betrieben ein erheblicher Anteil der Rohprotein- und Energieversorgung über das Grundfutter abgedeckt werden.

Unter Hoch-Zucker-Gräser werden solche Futtergräser verstanden, die einen genetisch deutlich höheren Gehalt an wasserlöslichen Kohlenhydraten und eine höhere Verdaulichkeit besitzen als vergleichbare Sorten. Dass es prinzipiell Unterschiede im Zuckerniveau zwischen verschiedenen Grasarten gibt, ist bekannt, beispielsweise weisen Weidelgräser allgemein einen höheren Zuckergehalt im Vergleich zu anderen wichtigen Grünlandgräsern wie Wiesenrispe, Wiesenlieschgras oder Knaulgras auf. Aber auch der weniger nutzungsintensive Wiesenschwingel ist durch höhere Zuckergehalte charakterisiert; sie liegen aber unter dem Niveau der Weidelgräser, beispielsweise kann Deutsches Weildelgras bis zu 30 \% wasserlöslicher Kohlenhydrate in der Trockenmasse enthalten (Greef et al., 2005). 
Durch die gezielte Züchtung auf hohe Zuckergehalte verspricht man sich nunmehr nennenswerte Unterschiede zwischen den Sorten einer Grasart. Erste Sorten liegen vor und der allgemeine Trend lässt eine deutliche Zunahme im Zuckergehalt im Vergleich zu den Kontrollsorten bei einem annähernd vergleichbaren Rohprotein- und Rohfasergehalt erkennen.

\section{Vorteile von Hoch-Zucker-Gräser:}

- Schnell verfügbare Energie in Form von wasserlöslichen Kohlenhydraten ist der begrenzende Faktor beim Aufbau von Mikrobenprotein im Pansen des Wiederkäuers (Rooke et al., 1987).

- Hohe Gehalte an wasserlöslichen Kohlenhydraten haben einen hohen Stellenwert für die Schmackhaftigkeit bei Beweidung und sind auch bei Schnittnutzung erwünscht. Gerade für die Erzeugung hochwertiger Silage muss ausreichend und möglichst natürliches Gärsubstrat im Ausgangsmaterial vorhanden sein (Jänicke, 2005).

- Es konnten deutliche Verbesserungen in der Umsetzung des Futterproteins zu Milchund Fleischeiweiß bei einer Verringerung der N-Ausscheidungen über Kot und Harn nachgewiesen werden (Miller et al., 2001).

- Die Veränderungen des Gehaltes an wasserlöslichen Kohlenhydraten im jahreszeitlichen Verlauf sowie im Tagesverlauf führten bei Beweidung von Hoch- ZuckerGras zu positiven Effekten auf die Milchproduktion (Miller et al., 2001) (Tab. 1).

- Hoch- Zucker-Gräser besitzen eine höhere Verdaulichkeit als vergleichbare Sorten bei gleicher Reifegruppe und gleicher Ploidiestufe (Eickmeyer, 2004).

- Bei Charolais-Stieren, die auf einer Weide mit einer Hoch-Zucker-Grassorte konnte eine um 25 \% höhere Lebendgewichtzunahme und 20 \% höhere Aufnahme pro Tag erzielt werden als bei einer Kontrollsorte (Eickmeyer, 2004).

- Erkenntnisse über die Gehalte an wasserlöslichen Kohlenhydraten ermöglichen, da sie Energiespeicher darstellen, Rückschlüsse auf die Überwinterungsfähigkeit (Matthes, 1986). Mit zunehmendem Gehalt von wasserlöslichen Kohlenhydraten steigt die Winterhärte an (Humphreys, 1989). 
Tab. 1: Effekt eines erhöhten Zuckergehaltes auf die Milchproduktion.

\begin{tabular}{|l|l|l|l|}
\hline Merkmal & $\begin{array}{l}\text { Normale } \\
\text { Sorte }\end{array}$ & $\begin{array}{l}\text { Hoch-Zucker } \\
\text { Sorte }\end{array}$ & $\begin{array}{l}\text { Standard } \\
\text { Abweichung }\end{array}$ \\
\hline Milchleistung (kg/Tag) & 12,6 & 15,3 & 0,65 \\
\hline $\begin{array}{l}\text { Milcheiweiß } \\
\text { Ertrag (g/Tag) }\end{array}$ & 434 & 528 & 22 \\
\hline $\begin{array}{l}\text { N-Ausscheidung über Urin } \\
\text { (g/Tag) }\end{array}$ & 100 & 71 & 5,0 \\
\hline
\end{tabular}

(nach Miller et al., 2001).

\section{Wasserlösliche Kohlenhydrate der Graspflanze}

Langkettige Kohlenhydrate, wie die Fuktane sind wichtige Langzeit-Reserven in den Gräser des gemäßigten Klimabereichs (Mitterer et al., 2002). Neben Fruktan bestehen WlK aus Glukose, Fruktose Saccharose und Stärke und Zählen zu den Nicht- struktur-Kohlenhydraten. Im Gegensatz dazu werden Hemizellulose, Zellulose und Pektin als Struktur-Kohlenhydrate bezeichnet (Van Soest et al., 1991; Schubiger et al., 1998).

WlK liegen im Zellinneren gelöst vor (Kühbauch, 1978), die strukturierten Kohlenhydrate sind Bestandteile der Zellwände (Bailey, 1973). Hinsichtlich des Gehaltes und der Verteilung der Kohlenhydrate bestehen zwischen den einzelnen Pflanzenorganen Unterschiede. Blätter haben niedrigere Gehalte als Blattscheiden und diese wiederum niedrigere als Stängel (Matthes, 1986). Das Hauptspeicherorgan der Graspflanzen für wasserlösliche Kohlenhydrate, und damit auch Fruktan, ist der Stängel (Fulkerson und Donaghy, 2001). Nach Alberda (1960) befanden sich im Deutschen Weidelgras die höchsten WlK- Gehalte im Bereich des Halmgrundes (Stoppelzone), die niedrigsten in den Wurzeln. Lediglich in Pflanzen, die Inulin speichern, sind Fruktane in den reproduktiven Geweben enthalten (Dahlhoff, 2003). Die Verteilungsreihenfolge der WlK kann sich allerdings im Verlauf der Pflanzenentwicklung ändern. Da die Bildung der WlK in den Blättern erfolgt, ist der WlK- Gehalt besonders in Zeiten hoher Assimilationsaktivität dort relativ hoch. Durch den Abtransport der Assimilate in die Speicherorgane Stängel und Blattscheiden kann sich jedoch das Schwergewicht der Konzentration in diese Pflanzenteile verlagern, sofern der Assimilateverbrauch nur gering ist (Matthes, 1986). Als sogenannter Zwischenspeicher werden die Fruktane während des vegetativen Wachstums im Blatt und vorwiegend im Stängel gebildet. Dazu werden ploymere Ketten aufgebaut, die sich möglicherweise sortenspezisch ausprägen können (Longland und Cairns, 2000). 
Nach Fulkerson und Donaghy (2001) ist das Ausmaß der Speicherung wasserlöslicher Kohlenhydrate von der Photosyntheserate einerseits und dem Verbrauch von Kohlenhydraten für Wachstum und Atmung der Pflanze andererseits abhängig. Wenn der Energiebedarf der Pflanze hoch ist, z. B. in Phasen schnellen Wachstums oder während der Blüte, sinkt der Fruktangehalt; umgekehrt steigt er bei reduziertem Wachstum und gleich bleibender Photosyntheseaktivität (Longland und Cairns, 2000).

\subsection{Fragestellung}

Ziel der vorliegenden Arbeit war es, experimentell zu ermitteln inwieweit Hoch-ZuckerGräsersorten die Verdaulichkeit und die Grundfutterqualität durch Verschiebungen der wasserlöslichen Kohlenhydrate beeinflussen.

Die folgenden Fragen sollten im Vergleich zu den Standardvarianten beantwortet werden:

\section{$\underline{\text { Sortenversuch }}$}

Wie verändern sich der Ertrag, die Futterqualitätsparameter (Verdaulichkeit, Rohprotein und Rohfaser) und wasserlösliche Kohlenhydrate - und Fruktangehalt in der Pflanzensubstanz verschiedener Futtergräser in Abhängigkeit von Schnittzeitpunkt, Standort und Vegetationsperiode?

Welche Beziehungen gibt es zwischen Rohprotein, Rohfaser und Verdaulichkeit und wasserlösliche Kohlenhydrate - Gehalt?

\section{$\underline{\text { Zeitreihenversuch }}$}

Wie stark werden die wasserlösliche Kohlenhydrate - und Fruktangehalt im Verlauf des ersten Aufwuchses von Pflanzensorte und N-Düngung in aufeinander folgenden Terminen und im diurnalen Rhythmus beeinflussen? Lassen sich sortenspezifische Unterschiede im Polymerisationsmuster der Fruktane feststellen? 


\section{Material und Methoden}

\subsection{Versuchsstandorte}

Der Sortenversuch wurde auf zwei Standorte angelegt.

1- Versuchsfeld des Institutes für Pflanzenbau und Grünlandwirtschaft der Bundesforschungsanstalt für Landwirtschaft (FAL) in Völkenrode (BS)

2- Versuchsfeld von Euro Grass Breeding Hof Steimke (HS)

Der Versuchstandort des Zeitreihenversuches, welcher die Untersuchung der Gesamtzuckerund Fruktangehalte im Verlauf des ersten Aufwuchses beinhaltete, befand sich auf dem Versuchsfeld des Institutes für Pflanzenbau und Grünlandwirtschaft der Bundesforschungsanstalt für Landwirtschaft (FAL) in Völkenrode.

Die Standortbeschreibungen des Sortenversuches und des Zeitreihenversuches sind in Tabelle 2 dargestellt.

Die Analyse der bodenchemischen Kennwerte (Phosphor-, Kalium- und Magnesiumgehalte) des Versuchsstandortes BS im Anlagejahr (2005) und im 2. Hauptversuchsjahr (2007) erfolgte am Institut für Pflanzenbau und Grünlandwirtschaft der Bundesforschungsanstalt für Landwirtschaft (FAL) in Völkenrode (Tab. 3). An den Versuchstandorten HS erfolgte die Analyse der bodenchemischen Kennwerte (Phosphor-, Kalium- und Magnesiumgehalte) nur im Anlagejahr (2005). 
Tab. 2: Standortbeschreibung des Sortenversuches an den zwei Versuchstandorten (BS und HS) und des Zeitreihenversuches.

\begin{tabular}{|c|c|c|}
\hline & Sortenversuch BS und Zeitreihenversuch & Sortenversuch HS \\
\hline Fläche & $\begin{array}{l}\text { Versuchsfeld des Institutes für Pflanzenbau und } \\
\text { Grünlandwirtschaft Völkenrode }\end{array}$ & $\begin{array}{l}\text { Versuchsfeld von } \\
\text { EuroGrass } \\
\text { Breeding } \\
\text { Hof Steimke }\end{array}$ \\
\hline $\begin{array}{l}\text { geographische } \\
\text { Lage }\end{array}$ & $\begin{array}{l}52^{\circ} 18^{\prime} \text { Nord } \\
10^{\circ} 27^{\prime} \text { Ost }\end{array}$ & $\begin{array}{l}52^{\circ} 46^{\prime} \text { Nord } 9^{\circ} 13^{\prime} \\
\text { Ost }\end{array}$ \\
\hline \begin{tabular}{|l|} 
geologische \\
Herkunft
\end{tabular} & Diluvium (Pleistozän, einschließlich Tertiär) & unbekannt \\
\hline Höhe über NN & $80 \mathrm{~m}$ & $40 \mathrm{~m}$ \\
\hline Bodenart & schluffiger Sand & $\begin{array}{l}\text { humoser stark } \\
\text { lehmiger Sand }\end{array}$ \\
\hline Humusgehalt & schwach humos & stark humos \\
\hline Bodentyp & Podsol, Braunerde & sandiger Lehm \\
\hline $\mathrm{pH}\left(\mathrm{CaCl}_{2}\right)$ & 5,8 & 5,5 \\
\hline Ackerzahl & 40 & 45 Bodenpunkte \\
\hline Vorfrucht & Mais & Wintergerste \\
\hline Aussaat & \begin{tabular}{|l|} 
21.04.05 Sortenversuch \\
22.09.05 Zeitreihenversuch
\end{tabular} & 22.04 .2005 \\
\hline
\end{tabular}

Tab. 3: pH-Wert, Phosphat- $\left(\mathrm{P}_{2} \mathrm{O}_{5}\right)$-, Kaliumoxid- $\left(\mathrm{K}_{2} \mathrm{O}\right)$ - und Magnesiumgehalte $(\mathrm{Mg})$ im lufttrockenen Boden (Probenahme im Februar).

\begin{tabular}{|c|c|c|c|c|c|c|c|c|}
\hline Versuchsstandort & \multirow{2}{*}{$\begin{array}{c}\mathrm{pH}-\text { Wert } \\
\text { und Tiefe }(\mathrm{cm})\end{array}$} & \multicolumn{2}{|c|}{$\mathrm{P}_{2} \mathrm{O}_{5}$} & \multicolumn{3}{c|}{$\mathrm{K}_{2} \mathrm{O}$} & \multicolumn{2}{c|}{$\mathrm{Mg}$} \\
\cline { 3 - 10 } & \multicolumn{2}{|c|}{} & \multicolumn{6}{|c|}{ mg/100 g Boden } \\
\hline BS 0-20 & 2005 & 2007 & 2005 & 2007 & 2005 & 2007 & 2005 & 2007 \\
\hline HS 0-20 & 5,8 & 5,8 & 17,0 & 7,2 & 12,9 & 6,6 & 3,6 & 4,9 \\
\hline
\end{tabular}




\subsection{Klima und Witterung}

\subsubsection{Versuchsstandort BS}

Im Anlagejahr 2005 lagen die Niederschläge im Januar und Februar im Bereich des langjährigen Mittels. Die Niederschläge lagen von März bis Dezember unter, außer Mai und Juli über dem langjährigen Mittel (Abb. 1). Die Temperaturen lagen im Anlagejahr 2005 von Januar bis Dezember über, Februar und August unter dem langjährigen Mittel (Abb. 2). Das 1. Hauptversuchsjahr (2006) war warm und trocken, besonders der Juli war durch eine extreme Trockenheit charakterisiert. Die Niederschläge lagen unter dem langjährigen Mittel, nur im August waren die Niederschläge viel höher als das langjährige Mittel (Abb. 1). Die Temperaturen lagen von Januar bis März unter dem langjährigen Mittel, von April bis Dezember lagen die Temperaturen über, im August unter dem langjährigen Mittel (Abb. 2).

Der Jahresniederschlag betrug nur 445,2 mm und lag so deutlich unter dem langjährigen Mittel (616,7 mm) (Tab. 4). Die Jahresdurchschnittstemperatur lag mit 10,3 ${ }^{\circ} \mathrm{C}$ über dem langjährigen Mittel (9,1 $\left.{ }^{\circ} \mathrm{C}\right)$ (Tab. 4). Das 2. Hauptversuchsjahr (2007) war zu nass und hatte erhöhte Niederschläge von Januar bis November außer April und Oktober. Die Niederschläge in den genannten Monten waren deutlich höher als das langjährige Mittel. Im April, Oktober und Dezember waren die Niederschläge niedriger als das langjährige Mittel (Abb. 1). Die Temperaturen lagen von Januar bis Juni über dem langjährigen Mittel, von Juli bis Dezember im Bereich des langjährigen Mittels (Abb. 2).

Der Jahresniederschlag betrug 867,6 mm und lag so deutlich über dem langjährigen Mittel (616,7 mm) (Tab. 4). Die Jahresdurchschnittstemperatur lag mit 10,6 ${ }^{\circ} \mathrm{C}$ über dem langjährigen Mittel $\left(9,1^{\circ} \mathrm{C}\right)$ (Tab. 4).

Tab. 4: Die Jahresdurchschnittstemperatur und Jahresniederschlag [mm] des Anlagejahres 2005 und der Hauptversuchsjahre 2006 und 2007 für die Versuchsstandorte BS und HS sowie das langjährige Mittel (1962-2005) für Versuchsstandort BS und (2005-2007) für Versuchsstandort HS.

\begin{tabular}{|c|c|c|c|c|}
\hline \multirow{2}{*}{ Versuchsjahr } & \multicolumn{2}{|c|}{ Temperatur $^{\circ} \mathrm{C}$} & \multicolumn{2}{c|}{ Niederschläge mm } \\
\cline { 2 - 5 } & BS & HS & BS & HS \\
\hline 2005 & 9,8 & 9,9 & 511 & 620,3 \\
\hline 2006 & 10,3 & 10,3 & 445,2 & 610,6 \\
\hline 2007 & 10,6 & 10,4 & 867,6 & 933,4 \\
\hline Langjähriges Mittel & 9,1 & 10,2 & 616,7 & 722 \\
\hline
\end{tabular}




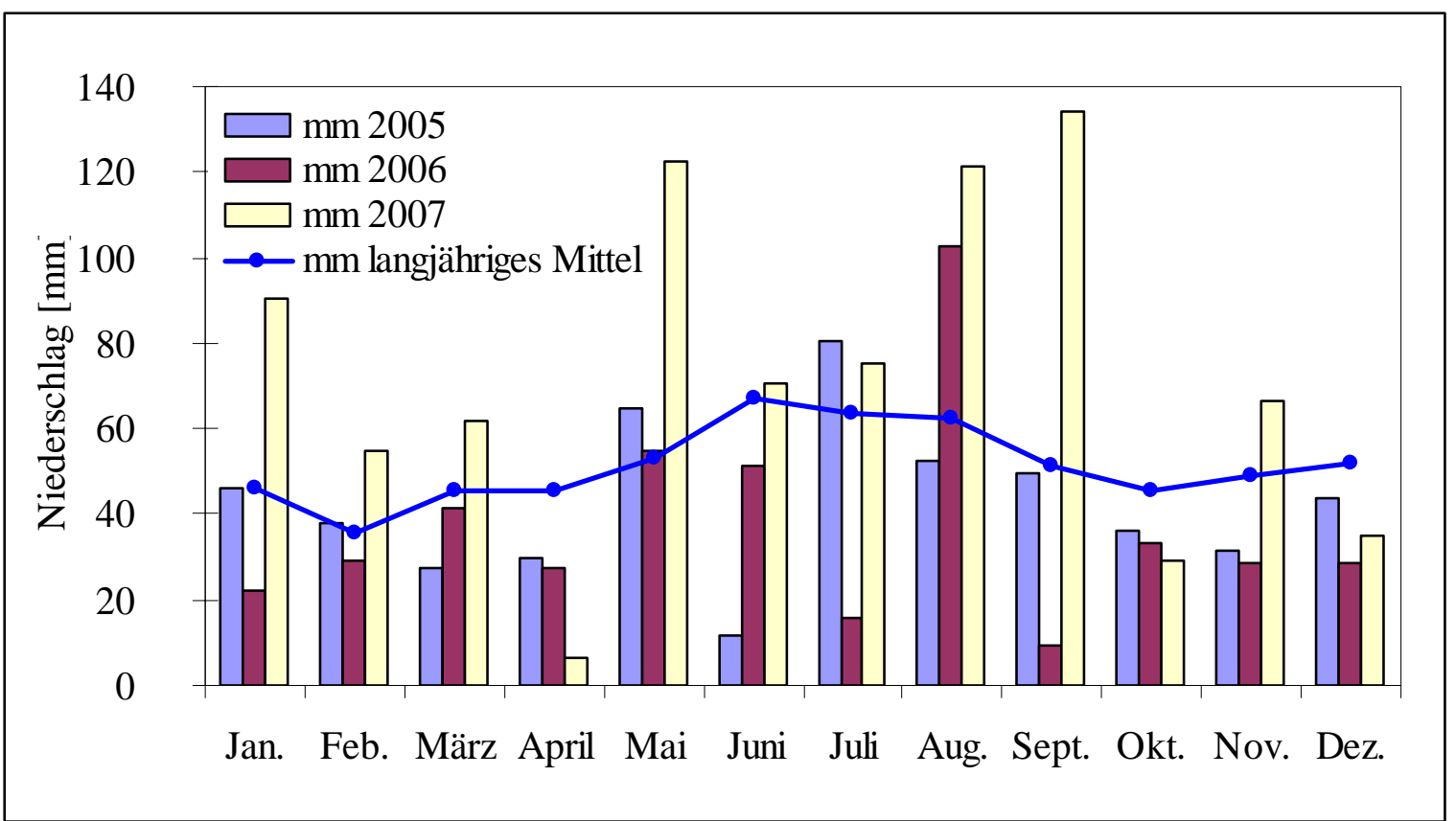

Abb. 1: monatliche Niederschlagssummen [mm] des Anlagejahres 2005 und der Hauptversuchsjahre 2006 und 2007 sowie das langjährige Mittel (1962-2005) für den Versuchsstandort Braunschweig.

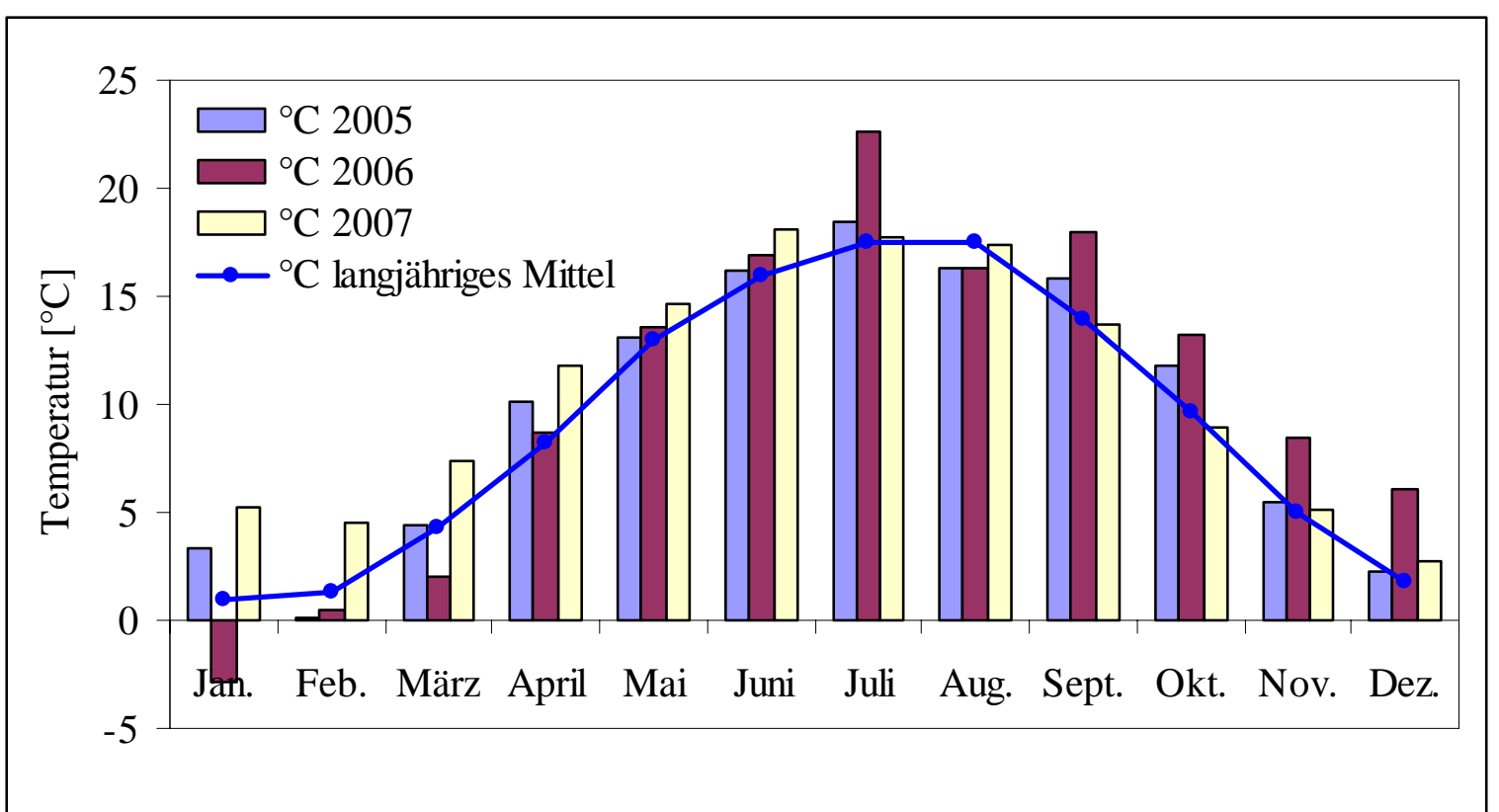

Abb. 2: Monatsmitteltemperaturen $\left[{ }^{\circ} \mathrm{C}\right]$ des Anlagejahres 2005 und der Hauptversuchsjahre 2006 und 2007 sowie das langjährige Mittel (1962-2005) für den Versuchsstandort Braunschweig. 


\subsubsection{Versuchsstandort HS}

Das langjährige Mittel für Versuchsstandort HS ist nicht vorhanden. Um einen Bezugspunkt zu bilden wurde das Mittel (Temperatur und Niedershlag) aus den Versuchsjahren (2005, 2006 und 2007) berechnet.

Im Anlagejahr 2005 lagen die Niederschläge von Januar bis Dezember außer Mai und Oktober unter dem langjährigen Mittel. Mai und Oktober lagen im Bereich des langjährigen Mittels (Abb. 3). Die Temperaturen lagen im Anlagejahr 2005 im Januar über dem langjährigen Mittel. Im Februar, Juli, November und Dezember lag Die Temperatur unter dem langjährigen Mittel. Von März bis Juni und von August bis Oktober lag Die Temperatur im Bereich des langjährigen Mittels (Abb. 4). Im 1. Hauptversuchsjahr (2006) lagen die Niederschläge unter dem langjährigen Mittel außer März, April, August und November, in den die Niederschläge über dem langjährigen Mittel lagen (Abb. 3). Die Temperaturen lagen von Januar bis April und im Juni unter dem langjährigen Mittel. Im Mai und August lagen Die Temperaturen im Bereich des langjährigen Mittels. Im Juli und von September bis Dezember lagen Die Temperaturen über dem langjährigen Mittel (Abb. 4).

Der Jahresniederschlag betrug im 2006 nur 610,6 mm und lag so deutlich unter dem langjährigen Mittel (722 mm) (Tab. 4). Die Jahresdurchschnittstemperatur lag mit 10,3 ${ }^{\circ} \mathrm{C}$ über dem langjährigen Mittel (10,2 ${ }^{\circ} \mathrm{C}$ ) (Tab. 4). Das 2. Hauptversuchsjahr (2007) war zu nass und hatte erhöhte Niederschläge von Januar bis Dezember außer April und August. Die Niederschläge in den genannten Monten waren deutlich höher als das langjährige Mittel. Im April und August waren die Niederschläge niedriger als das langjährige Mittel (Abb. 3). Die Temperaturen lagen im 2007 von Januar bis Juni und im August über dem langjährigen Mittel. Im Juli und von September bis Dezember lagen die Temperaturen unter dem langjährigen Mittel (Abb. 3).

Der Jahresniederschlag betrug 933,4 mm und lag so deutlich über dem langjährigen Mittel (722 mm) (Tab. 4). Die Jahresdurchschnittstemperatur lag mit 10,4 ${ }^{\circ} \mathrm{C}$ über dem langjährigen Mittel (10,2 $\left.{ }^{\circ} \mathrm{C}\right)$ (Tab. 4). 


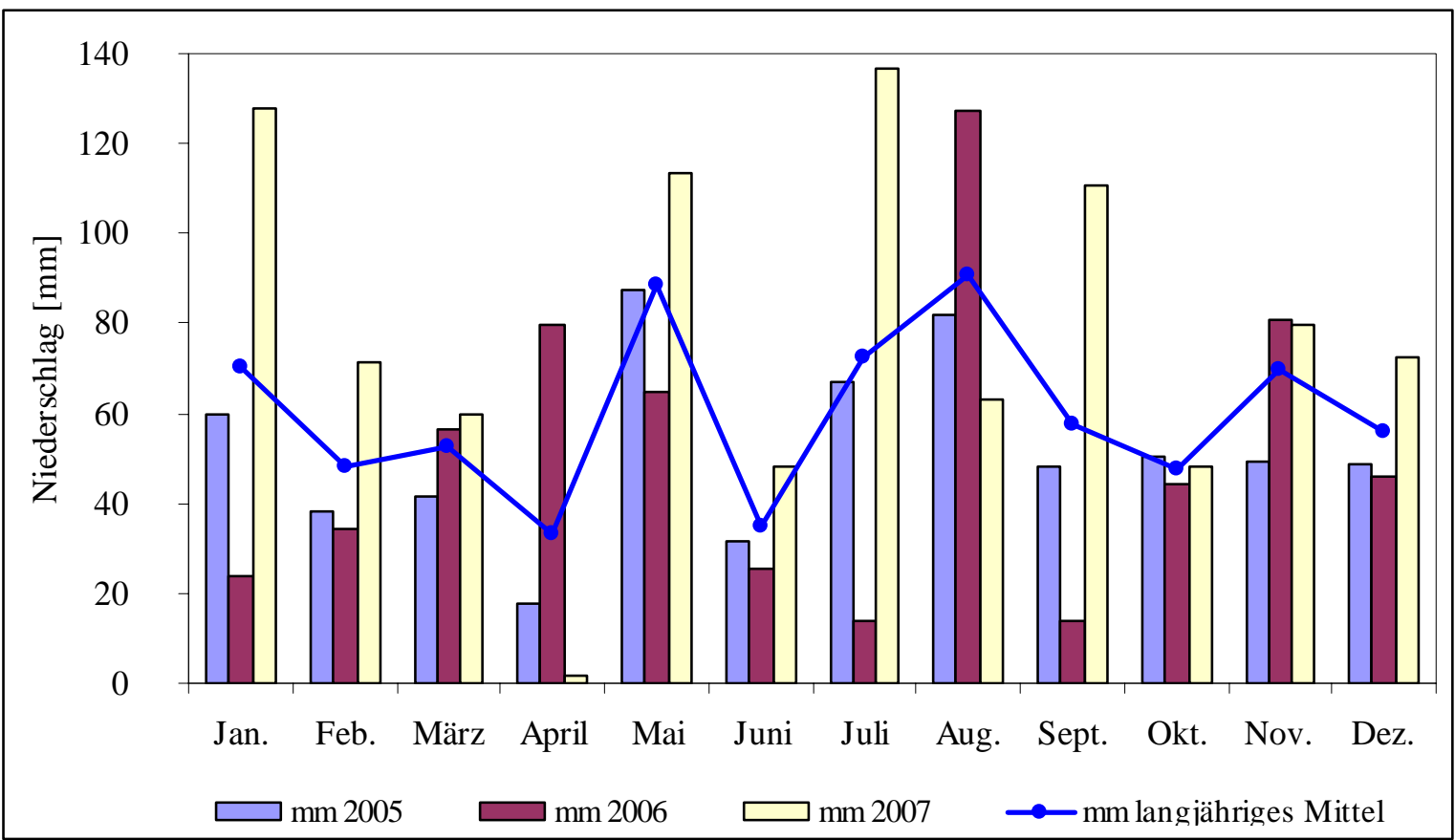

Abb. 3: Monatliche Niederschlagssummen [mm] des Anlagejahres 2005 und der Hauptversuchsjahre 2006 und 2007 sowie das langjährige Mittel (2005-2007) für den Versuchsstandort Hof Steimke.

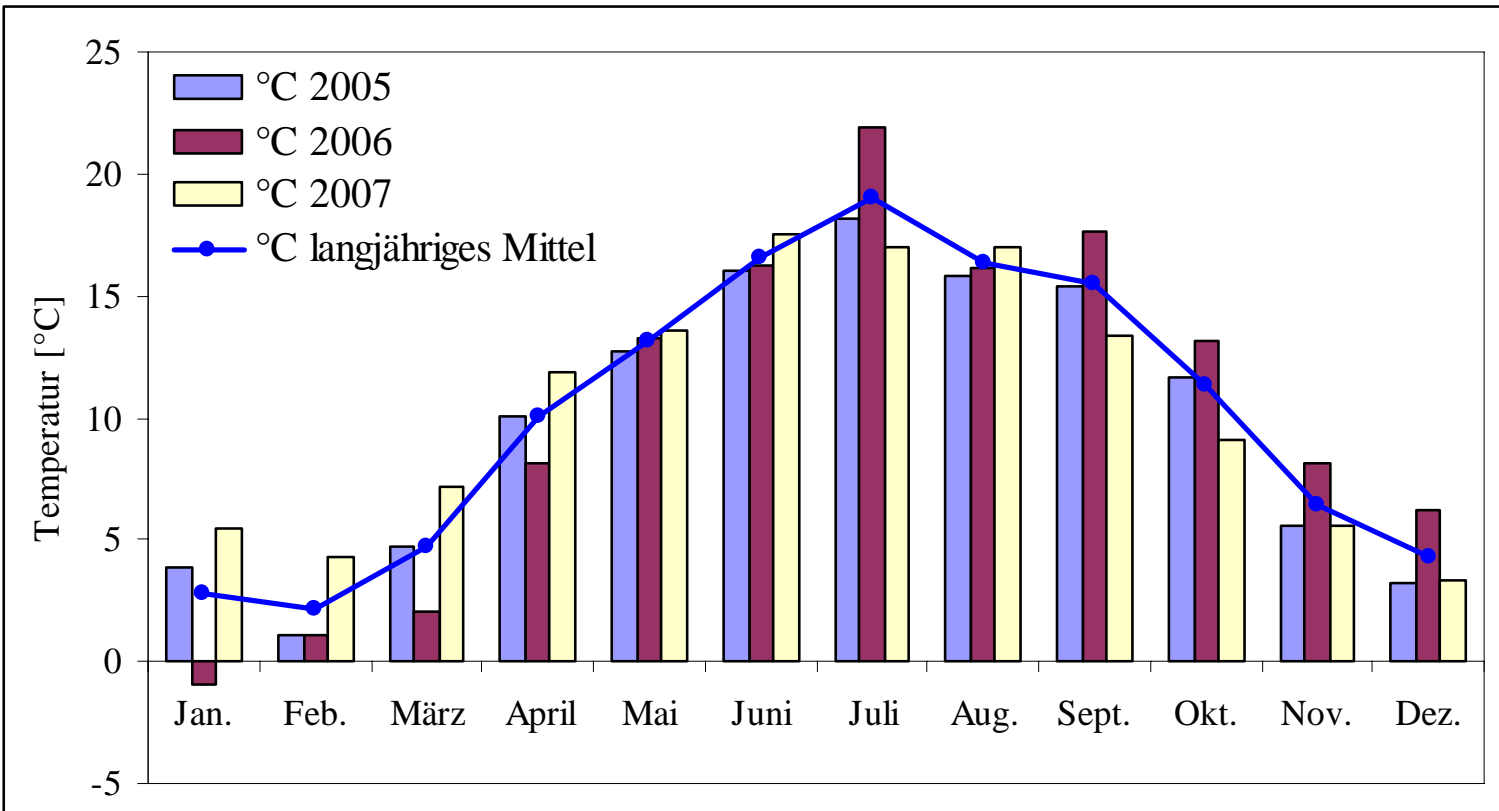

Abb. 4: Monatsmitteltemperaturen $\left[{ }^{\circ} \mathrm{C}\right]$ des Anlagejahres 2005 und der Hauptversuchsjahre 2006 und 2007 sowie das langjährige Mittel (2005-2007) für den Versuchsstandort Hof Steimke. 


\subsection{Versuchsanlage}

\subsubsection{Sortenversuch}

Die Veränderungen in den wasserlösliche kohlenhydrategehalten der verschiedenen Futtergräser wurden an 20 Sorten aus den Reifegruppen (früh, mittel und spät) und den Ploidiegruppen (Di- und Tetraploidie) untersucht (Tab. 5). Die geprüften Sorten wurden in Reinsaat mit den Saatstärken von $25 \mathrm{~kg} / \mathrm{ha}$ bei diploiden Sorten und $30 \mathrm{~kg} / \mathrm{ha}$ bei tetraploiden Sorten an zwei Versuchsstandorten (BS und HS) angebaut. Die Ansaat erfolgte im April 2005 an den Standorten BS und HS mit einer Parzellendrillmaschine (Firma Wintersteiger). Die Parzellengröße betrug für alle Standorte $11,9 \mathrm{~m}^{2}$ (8,5 m * 1,4 m).

Die Feldversuchsanlage des Sortenversuches bestand an allen Versuchsstandorten (BS und HS) aus einer einfaktoriellen randomisierten Anlage mit 2 Schnitterminen (Früh- und Spätschnitt), 20 Sorten und drei Wiederholungen (Abb. 5).

An den allen Versuchsstandorten wurden 3 Vegetationsjahre erfasst. Das erste Versuchsjahr (2005) wurde als Ansaatjahr berücksichtigt, in dem 3 Schnitte durchgeführt wurden. In den Hauptversuchsjahren 2006 und 2007 wurden in BS 5 Schnitte und in HS 4 Schnitte durchgeführt (Tab. 8).

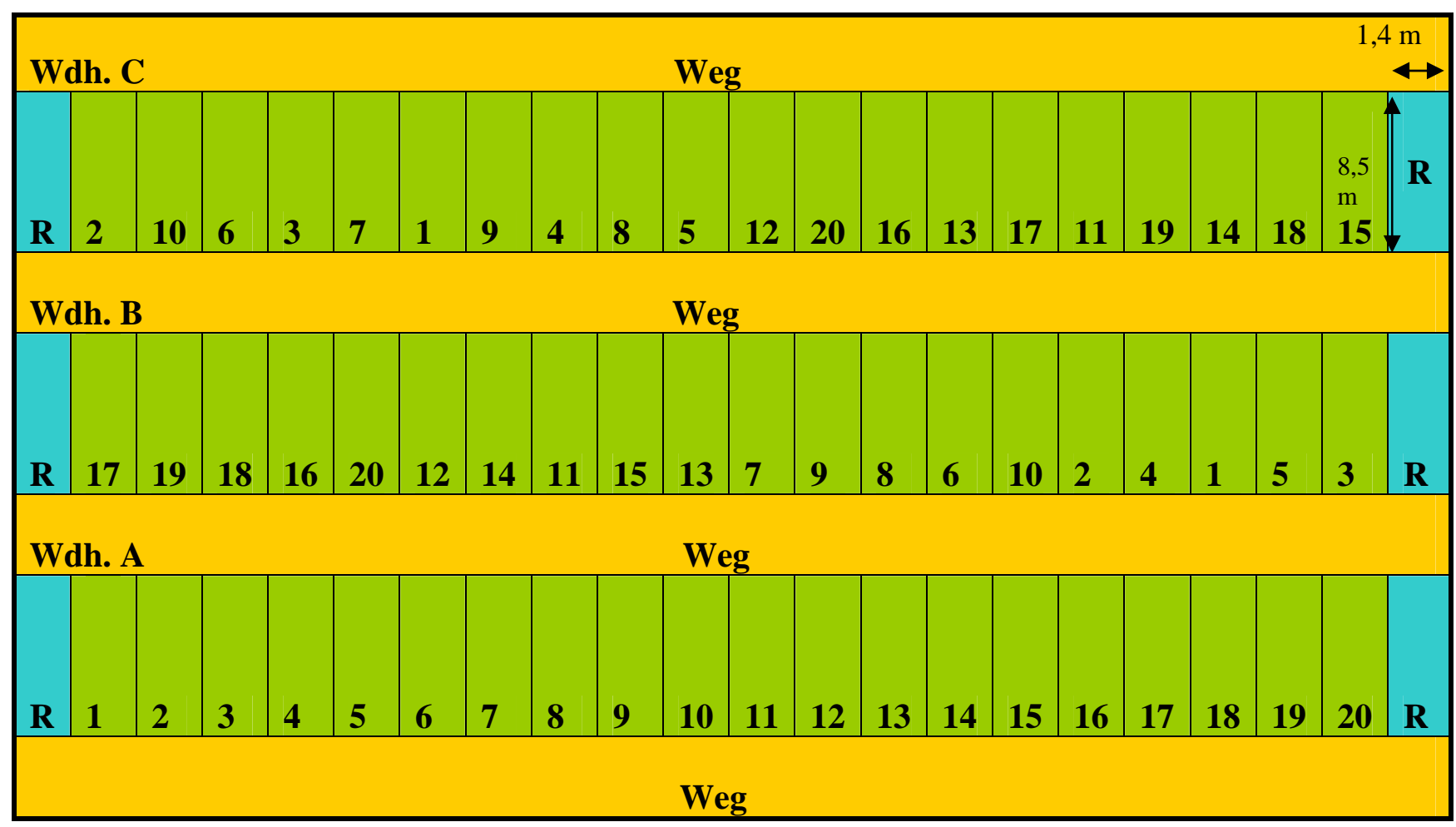

Abb. 5: Versuchsanlage des Sortenversuches.

$\mathrm{R}=$ Rand, $\mathrm{Wdh}=$ Wiederholung 
Tab. 5: Verwendete Futtergräsersorten für den Sortenversuch (Züchter, Lateinischer Name, Deutscher Name, Ploidiegrugge, wasserlösliche kohlenhydrategehalt (Wlk-Ghelat) und Reifegruppe).

\begin{tabular}{|c|c|c|c|c|c|c|c|}
\hline Nr. & Sorte & Züchter & $\begin{array}{c}\text { Lateinischer } \\
\text { Name }\end{array}$ & $\begin{array}{l}\text { Deutscher } \\
\text { Name }\end{array}$ & Ploidie & $\begin{array}{l}\text { WlK- } \\
\text { Gehalt }^{* *}\end{array}$ & $\begin{array}{l}\text { Reife- } \\
\text { gruppe }\end{array}$ \\
\hline 1 & Licarta $^{*}$ & & $\begin{array}{l}\text { Lolium } \\
\text { perenne }\end{array}$ & $\begin{array}{c}\text { Deutsches } \\
\text { Weidelgras }\end{array}$ & Diploid & Niedrig & früh \\
\hline 2 & Abersilo & IGER & $\begin{array}{l}\text { Lolium } \\
\text { perenne }\end{array}$ & $\begin{array}{c}\text { Deutsches } \\
\text { Weidelgras }\end{array}$ & Diploid & Hoch & früh \\
\hline 3 & $\mathrm{EGB}^{*}{ }^{*}$ & EGB & $\begin{array}{l}\text { Lolium } \\
\text { perenne }\end{array}$ & $\begin{array}{c}\text { Deutsches } \\
\text { Weidelgras }\end{array}$ & Diploid & Hoch & früh \\
\hline 4 & Respect $^{*}$ & & $\begin{array}{l}\text { Lolium } \\
\text { perenne }\end{array}$ & $\begin{array}{c}\text { Deutsches } \\
\text { Weidelgras }\end{array}$ & Diploid & Niedrig & mittel \\
\hline 5 & Betty & & $\begin{array}{l}\text { Lolium } \\
\text { perenne }\end{array}$ & $\begin{array}{l}\text { Deutsches } \\
\text { Weidelgras }\end{array}$ & Diploid & Hoch & mittel \\
\hline 6 & Aberdart & IGER & $\begin{array}{l}\text { Lolium } \\
\text { perenne }\end{array}$ & $\begin{array}{c}\text { Deutsches } \\
\text { Weidelgras }\end{array}$ & Diploid & Hoch & mittel \\
\hline 7 & Rodrigo* & & $\begin{array}{l}\text { Lolium } \\
\text { perenne }\end{array}$ & $\begin{array}{l}\text { Deutsches } \\
\text { Weidelgras }\end{array}$ & Diploid & Hoch & mittel \\
\hline 8 & EGB2 & EGB & $\begin{array}{l}\text { Lolium } \\
\text { perenne }\end{array}$ & $\begin{array}{c}\text { Deutsches } \\
\text { Weidelgras }\end{array}$ & Diploid & Niedrig & mittel \\
\hline 9 & Aubisque & & $\begin{array}{l}\text { Lolium } \\
\text { perenne }\end{array}$ & $\begin{array}{c}\text { Deutsches } \\
\text { Weidelgras }\end{array}$ & Tetraploid & Niedrig & mittel \\
\hline 10 & Sponsor & & $\begin{array}{l}\text { Lolium } \\
\text { perenne }\end{array}$ & $\begin{array}{c}\text { Deutsches } \\
\text { Weidelgras }\end{array}$ & Diploid & Niedrig & spät \\
\hline 11 & AberAvon* & IGER & $\begin{array}{l}\text { Lolium } \\
\text { perenne }\end{array}$ & $\begin{array}{c}\text { Deutsches } \\
\text { Weidelgras }\end{array}$ & Diploid & Hoch & spät \\
\hline 12 & EGB3 & EGB & $\begin{array}{l}\text { Lolium } \\
\text { perenne }\end{array}$ & $\begin{array}{c}\text { Deutsches } \\
\text { Weidelgras }\end{array}$ & Diploid & Niedrig & spät \\
\hline 13 & EGB4 & EGB & $\begin{array}{l}\text { Lolium } \\
\text { perenne }\end{array}$ & $\begin{array}{c}\text { Deutsches } \\
\text { Weidelgras }\end{array}$ & Diploid & Hoch & spät \\
\hline 14 & EGB5 & EGB & $\begin{array}{l}\text { Lolium } \\
\text { perenne }\end{array}$ & $\begin{array}{c}\text { Deutsches } \\
\text { Weidelgras }\end{array}$ & Tetraploid & unbekannt & spät \\
\hline 15 & Bocage & & $\begin{array}{l}\text { Lolium } \\
\text { perenne }\end{array}$ & $\begin{array}{c}\text { Deutsches } \\
\text { Weidelgras } \\
\end{array}$ & Tetraploid & Hoch & spät \\
\hline 16 & Liherold & & $\begin{array}{c}\text { Festuca } \\
\text { pratensis }\end{array}$ & $\begin{array}{c}\text { Wiesen- } \\
\text { schwingelgras }\end{array}$ & Diploid & Niedrig & früh \\
\hline 17 & Litornado & & $\begin{array}{c}\text { Festuca } \\
\text { pratensis }\end{array}$ & $\begin{array}{c}\text { Wiesen- } \\
\text { schwingelgras }\end{array}$ & Tetraploid & Hoch & früh \\
\hline 18 & Lifema & & $\begin{array}{c}\text { Festulolium. } \\
\text { sp. }\end{array}$ & $\begin{array}{c}\text { Wiesen- } \\
\text { schweidelgras }\end{array}$ & Tetraploid & unbekannt & früh \\
\hline 19 & Lidaglo & & $\begin{array}{l}\text { Dactylis } \\
\text { glomerata }\end{array}$ & Knaulgras & unbekannt & unbekannt & früh \\
\hline 20 & Lischka & & $\begin{array}{l}\text { Phleum } \\
\text { pratense }\end{array}$ & $\begin{array}{l}\text { Wiesenliesch- } \\
\text { gras }\end{array}$ & unbekannt & unbekannt & mittel \\
\hline
\end{tabular}

* = Ausgewählte Sorten für den Zeitreihenversuch (insgesamt 5)

** = Quelle: nach Eurograss 
Tab. 6: Schnitttermine im Sortenversuch und allen Versuchsstandorten BS und HS.

\begin{tabular}{|c|c|c|c|c|c|}
\hline \multirow{3}{*}{ Versuchsjahr } & \multirow{3}{*}{$\begin{array}{l}\text { Schnitt } \downarrow \\
\text { Schnittzeitpunkt } \\
\stackrel{\longrightarrow}{\longrightarrow}\end{array}$} & \multicolumn{4}{|c|}{ Versuchsstandort } \\
\hline & & \multicolumn{2}{|c|}{ BS } & \multicolumn{2}{|c|}{ HS } \\
\hline & & $\begin{array}{l}\text { Früh- } \\
\text { schnitt }\end{array}$ & $\begin{array}{l}\text { Spät- } \\
\text { schnitt }\end{array}$ & $\begin{array}{l}\text { Früh- } \\
\text { schnitt }\end{array}$ & $\begin{array}{l}\text { Spät- } \\
\text { schnitt }\end{array}$ \\
\hline \multirow{3}{*}{2005} & 1. Schnitt & 14.07 & 03.08 & 04.07 & 19.07 \\
\hline & 2. Schnitt & 16.08 & 05.09 & 03.08 & 17.08 \\
\hline & 3. Schnitt & 19.09 & 06.10 & 01.09 & 13.09 \\
\hline \multirow{5}{*}{2006} & 1. Schnitt & 23.05 & 07.06 & 15.05 & 26.05 \\
\hline & 2. Schnitt & 28.06 & 12.07 & 27.06 & 11.07 \\
\hline & 3. Schnitt & 25.07 & 09.08 & 01.08 & 16.08 \\
\hline & 4. Schnitt & 23.08 & 06.09 & 05.10 & 26.10 \\
\hline & 5. Schnitt & 19.09 & 04.10 & & \\
\hline \multirow{5}{*}{2007} & 1. Schnitt & 16.05 & 01.06 & 15.05 & 01.06 \\
\hline & 2. Schnitt & 13.06 & 03.07 & 25.06 & 03.07 \\
\hline & 3. Schnitt & 11.07 & 31.07 & 25.07 & 15.08 \\
\hline & 4. Schnitt & 14.08 & 28.08 & 14.09 & 24.09 \\
\hline & 5. Schnitt & 09.10 & 23.10 & & \\
\hline
\end{tabular}

\subsubsection{Zeitreihenversuch}

In diesem Versuch wurden der Gesamtzucker- und der Fruktangehalt im Verlauf des ersten Aufwuchses untersucht. 5 Sorten wurden dazu aus den Sortenversuchsergebnissen des Ansaatsjahres (2005) ausgewählt (s. Tab. 10).

Die ausgewählte 5 Sorten wurden in Reinsaat mit einer Saatstärke von $25 \mathrm{~kg} / \mathrm{ha}$ in einfacher Wiederholung am 22.09.2005 mit zwei N-Düngungsvarianten auf dem Versuchsfeld des Institutes für Pflanzenbau und Grünlandwirtschaft der Bundesforschungsanstalt für Landwirtschaft (FAL) in Völkenrode angelegt. Die Parzellengröße betrug 46,2 m² (33*1,4) (Abb. 6).

Der Zeitreihenversuch wurde in 2 Vegetationsjahren (2006 und 2007) durchgeführt.

Die Düngungsvarianten sind im Versuchsjahr 2006: -Normaldüngung 80 kg/ha zum 1. Schnitt und zum 2.- und 3. Schnitt je $60 \mathrm{~kg} / \mathrm{ha}$. -Niedrigdüngung $30 \mathrm{~kg} / \mathrm{ha}$ zum 1 . Schnitt und zum 2.und 3. Schnitt je 10 kg/ha. Im Versuchsjahr 2007 wurde der Versuch nur mit Normaldüngung durchgeführt. N-Düngung ist Kalkammonsalpeter (KAS). 


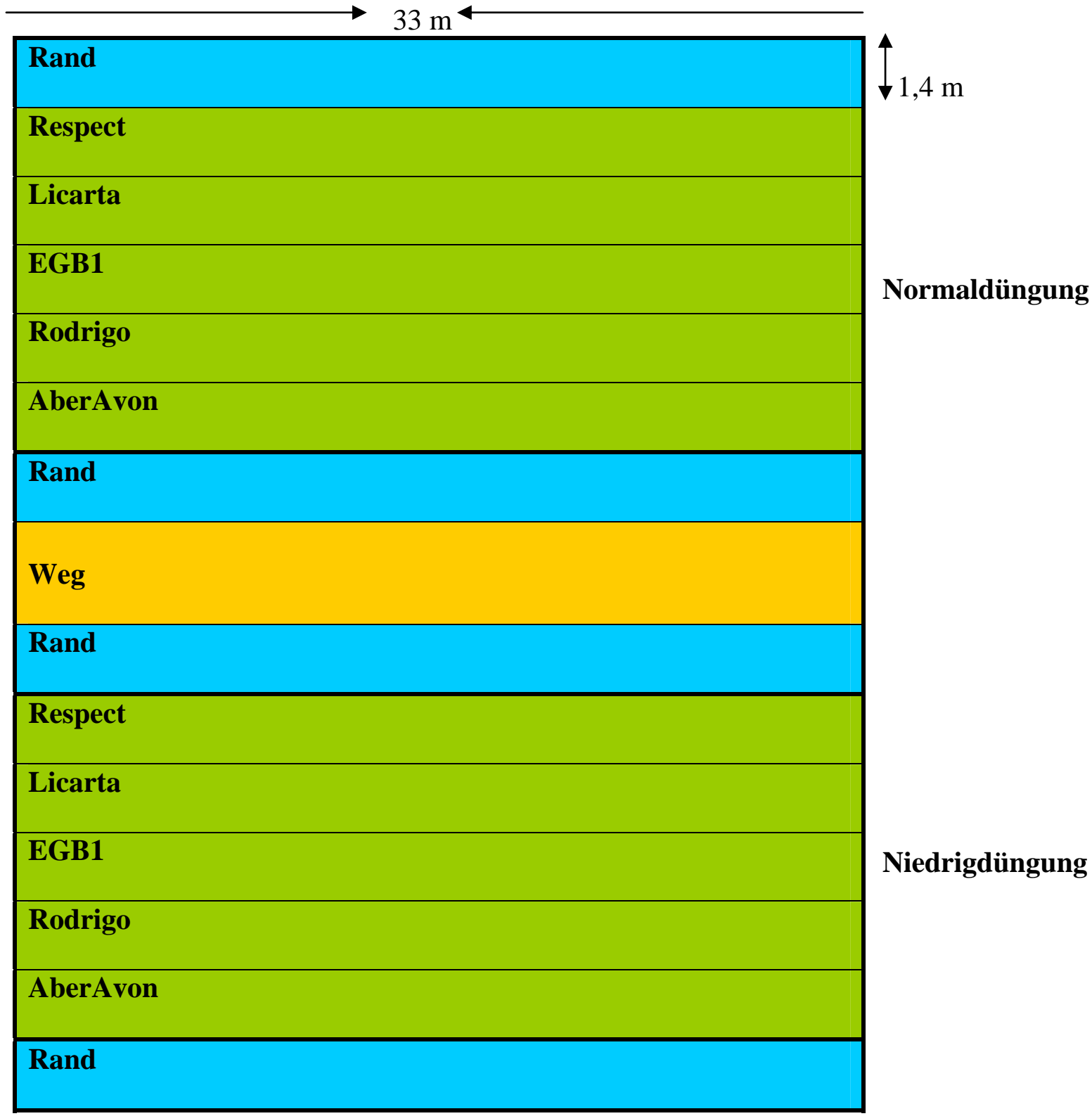

Abb. 6: Versuchsanlage des Zeitreihenversuches.

\subsection{Düngung und Pflege}

\subsubsection{Grunddüngung}

Der Sortenversuchsstandort BS und der Zeitreihenversuch wurden mit dem Grunddünger Thomaskali im Winter des Anlagejahres (9.02.05) mit 750 kg/ha, mit dem Grunddünger Kornkali im Winter des 1. Hauptversuchsjahres (25.01.06) mit 400 kg/ha und dem Grunddünger Thomaskali im Winter des 2. Hauptversuchsjahres (8.03.07) mit $1000 \mathrm{~kg} / \mathrm{ha}$ versorgt. Die Bestandteile des Thomaskali Phosphat, Kaliumoxid und Magnesiumoxid lagen im Verhältnis 7:21:3 vor. Die Bestandteile des Kornkalis Kaliumoxid und Magnesiumoxid lagen im Verhältnis 40:6 vor. 
Der Sortenversuchsstandort HS wurde im Winter des Anlagejahres 2005 mit 21,8 kg/ha P und 99,6 kg/ha K und im Winter des 2. Hauptversuchjahres 2007 mit 19,6 kg/ha P und 112 $\mathrm{kg} / \mathrm{ha} \mathrm{K}$ versorgt.

\subsubsection{N-Düngung}

Der Sortenversuchsstandort BS wurde im Verlauf der Vegetationsperiode mit KAS mit der Startgabe $50 \mathrm{~kg} / \mathrm{ha}$ am 17.05.05, mit $40 \mathrm{~kg} / \mathrm{ha}$ zum 1. Schnitt, mit $60 \mathrm{~kg} / \mathrm{ha}$ zum 2. Schnitt und mit 50 kg/ha zum 3. Schnitt im Anlagejahr 2005 gedüngt. Im 1., und 2. Hauptversuchjahre 2006 und 2007 wurde mit $80 \mathrm{~kg} /$ ha zum 1. Schnitt und mit $60 \mathrm{~kg} / \mathrm{ha}$ zum 2.- bis 5 . Schnitt versorgt.

Der Sortenversuchsstandort HS wurde im Verlauf der Vegetationsperiode mit KAS mit 80 $\mathrm{kg} / \mathrm{ha}$ zum 1. Schnitt, mit $65 \mathrm{~kg} / \mathrm{ha}$ zum 2. Schnitt, mit $50 \mathrm{~kg} / \mathrm{ha}$ zum 3. Schnitt und mit 40 kg/ha zum 4. Schnitt im 1. Hauptversuchjahr 2006 versorgt. Im 2. Hauptversuchjahr 2007 wurde mit $80 \mathrm{~kg} / \mathrm{ha}$ zum 1 . Schnitt, mit $70 \mathrm{~kg} /$ ha zum 2.- und 3. Schnitt und mit $50 \mathrm{~kg} / \mathrm{ha}$ zum 4. Schnitt versorgt.

N-Düngung erfolgte in der ersten Woche nach dem Schnitt.

Der Zeitreihenversuch, wie in der Beschreibung der Versuchsanlage erwähnt wurde, wurde im Versuchjahr 2006 mit KAS bei der Normaldüngung mit 80 kg/ha zum 1. Schnitt und zum 2.- und 3. Schnitt je $60 \mathrm{~kg} / \mathrm{ha}$ versorgt. Bei Niedrigdüngung wurde mit $30 \mathrm{~kg} / \mathrm{ha}$ zum 1 . Schnitt und zum 2.- und 3. Schnitt je $10 \mathrm{~kg} / \mathrm{ha}$ versorgt. Im Versuchsjahr 2007 wurde der Zeitreihenversuch mit KAS mit $80 \mathrm{~kg} / \mathrm{ha}$ zum 1. Schnitt und zum 2.- und 3. Schnitt je 60 $\mathrm{kg} / \mathrm{ha}$ versorgt (Normaldüngung).

Kalkammonsalpeter ist ein Stickstoffdünger mit $26 \%$ Stickstoff und $10 \%$ Kalzium. Er besteht zu von $74 \%$ aus Ammoniumnitrat $\left(\mathrm{NH}_{4} \mathrm{NO}_{3}\right)$ und $\mathrm{zu} 26 \%$ Kalziumkarbonat $\left(\mathrm{CaCO}_{3}\right)$.

\subsubsection{Pflege}

Die Parzellen wurden auf dem Sortenversuchsstandort BS im Anlagejahr von Hand nach dem Aufgang der Gräser von allen nicht gesäten Pflanzen befreit. Am (16.06.05) wurden die nicht gesäten Pflanzen mit Certrol B (Firma Spiess-Urania Chemicals GmbH) 1,5 1/ha und U46 DFluid (Firma BASF) 2 1/ha gespritzt. Die Parzellenzwischenräume wurden im Frühjahr und Herbst mit Roundup ${ }^{\circledR}$ (Firma Monsanto) gespritzt. Die Wege zwischen den Blöcken wurden bei jedem Schnitt gemäht. 
Die Parzellen auf dem Sortenversuchsstand HS wurden gegen alle nicht gesäten Pflanzen mit Ethophumesat 1,5 l/ ha (gegen Ungräser) und Starane ranger mit 2,5 l/ ha gespritzt.

Die Parzellenzwischenräume des Zeitreihenversuches wurden im Frühjahr und Herbst mit Roundup ${ }^{\circledR}$ (Firma Monsanto) gespritzt.

Beim Sortenversuchsstandort BS wurde im Herbst des 1. Hauptversuchsjahres eine Vielzahl

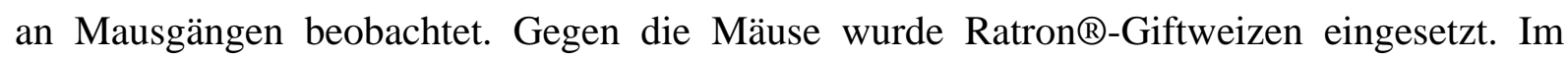
Sortenversuchsstandort HS wurde gegen die Mäuse Polytanol benutzt.

Der Sortenversuchsstandort BS wurde im Anlagejahr 2005 mit 20 mm (22.06.05), 35 mm (30.06.05) und $35 \mathrm{~mm}$ (07.07.05), im 1. Hauptversuchsjahr mit $30 \mathrm{~mm}$ (06.07.06), $25 \mathrm{~mm}$ (13.07.06), $30 \mathrm{~mm}$ (26.07.06) und $15 \mathrm{~mm}$ (20.09.06) beregnet.

Die Sortenversuchsstandort HS wurde nicht beregnet.

Der Zeitreihenversuch wurde im 1. Versuchsjahr 2006 mit 15 mm (20.07.06) und im 2. Versuchsjahr 2007 mit 40 mm (02.05.07) beregnet.

\subsection{Pflanzenbauliche Untersuchungen}

\subsubsection{Sortenversuch}

Der Schnitttermin des 1. Schnittes der Frühschnittvariante richtete sich nach dem Reifegrad der Sorte Respect (Beginn des Ähren- bzw. Rispenschiebens, definiert als „Siloreife“, wenn bei ca. 25\% der Pflanzen sind die Blütenstände eben sichtbar sind) (ca. 53 Tage nach dem 1. April). Der Schnitttermin des 1. Schnittes der Spätschnittvariante erfolgte dann ca. 14 Tage später (Blühbeginn, ca. 10 Pflanzen zeigen erste Blüten). Die Folgeschnitte wurden im 4 Wochen Rhythmus beerntet. Jede Parzelle wurde bei einer Schnitthöhe von $5 \mathrm{~cm}$ beerntet.

Die Probenahme der einzelnen Schnitte erfolgte zur gleichen Tageszeit jeweils 20 Sorten, die sowohl Vertreter der drei Reifegruppen früh, mittel und spät als auch der beiden Ploidiestufen di- und tetraploid sind.

$\mathrm{Zu}$ jedem Schnitttermin wurden Trockenmasseerträge (TM-Erträge) von jeder Parzelle ermittelt. Dazu wurde die gesamte Frischmasse der Parzelle mit der auf dem Grünfutterernter (Firma Haldrup) installierten Waage ermittelt. Zur Berechnung des TM-Ertrages wurde der Trockensubstanzgehalt bestimmt, dazu wurde das Pflanzenmaterial bis zur Gewichtskonstanten bei $105^{\circ} \mathrm{C}$ getrocknet. 


\section{Qualitätsuntersuchungen}

Für die Nah-Infrarot-Reflexions-Spektroskopie (NIRS) wurden Proben bei $60{ }^{\circ} \mathrm{C}$ getrocknet und anschließend auf $1 \mathrm{~mm}$ mit der Brabendermühle vermahlen. Für die chemische Analyse wurde (nur des Standortes BS im 1. Hauptversuchsjahr 2006) jeweils eine Probe der 5 Sorten des Zeitreihenversuches gefriergetrocknet und anschließend auf $1 \mathrm{~mm}$ mit der Brabendermühle vermahlen.

Anhand der gewonnenen Grasproben sollten mit NIRS folgende Parameter geschätzt werden: Gesamtzucker- und Fruktangehalt, organische Substanz, Rohprotein und Rohfaser.

Im Labor wurden mit HPLC (High Performance Liquid Chromatography) der Firma Kontron Instuments die Gesamtzucker (Wasserlöslicher Kohlenhydrate) und ihre Komponenten bestimmt.

\section{Nah-Infrarot-Reflexions-Spektroskopie}

Die Messung aller getrockneten und gemahlenen Pflanzenproben mit der Nah-InfrarotReflexions-Spektroskopie (NIRS) ermöglichte eine Reduktion der nasschemischen Laboruntersuchungen. Die Messungen wurden mit dem NIRS-Gerät Modell 5000 der Firma Foss NIRSystems und dem Messprogramm NIRS2, Version 4.01, der Firma Infrasoft International Inc. (1996) durchgeführt. Bei einem Spektrum von 1100 bis 2498nm wurden die Proben in 2nm Schritten und in 32facher Wiederholung gemessen. Die Verrechnung erfolgte mit dem Programm WinISI II, Version 1.50.

Das Kalibrationsset basiert auf der Referenzanalyse der nasschemischen Laboruntersuchungen des Instituts für Pflanzenbau und Grünlandwirtschaft der Bundesforschungsanstalt für Landwirtschaft. Anhand des H-Testes in der ISI Routine wurden einzelne Proben (15\% des Gesamtprobenumfanges) aus den Spektren gefiltert und naßchemisch referenziert.

Die Kalibrations- und Kreuzvalidierungsstatistiken für die einzelnen Parameter sind in Tabelle 7 angegeben. 
Tab. 7: NIRS Kalibrationen, erstellt aus dem Probenmaterial der Erntejahre 1994 bis 2001 für RP, RF, WIK, EULOS und RA und der Erntejahre 2002 bis 2005 für Fruktan.

\begin{tabular}{|c|c|c|c|c|c|c|c|}
\hline Inhaltsstoffe & $\mathbf{N}$ & Mean & SD & SEC & $\mathbf{R}^{\mathbf{2}}$ & SECV & 1-VR \\
\hline RP & 780 & 18,4 & 5,6 & 0,44 & 0,99 & 0,47 & 0,99 \\
\hline RF & 799 & 22,88 & 4,32 & 0,72 & 0,97 & 0,76 & 0,97 \\
\hline WlK & 296 & 11,49 & 5,12 & 0,65 & 0,98 & 0,77 & 0,98 \\
\hline EULOS & 188 & 256,9 & 76,4 & 19,8 & 0,93 & 24,1 & 0,9 \\
\hline RA & 127 & 9,52 & 1,71 & 0,74 & 0,81 & 0,85 & 0,76 \\
\hline Fruktan & 230 & 3,78 & 2,77 & 0,66 & 0,94 & 0,79 & 0,92 \\
\hline
\end{tabular}

N: Anzahl der Proben; Mean: durchschnittlicher Gehalt; SD: Standardabweichung; SEC: Standardfehler der Kalibrierung; $\mathrm{R}^{2}$ :Bestimmtheitsmaß; SECV: Standardfehler der Kreuzvalidierung; 1-VR: Bestimmtheitsmaß der Kreuzvalidierung.

\section{Bestimmung der enzymlösbaren organischen Substanz (ELOS)}

Die Untersuchung der Enzymlöslichkeit der organischen Masse von Futtermitteln nach der VDLUFA Verbandsmethode (VDLUFA, 1997) ist ein Verfahren zur Schätzung der in vivoVerdaulichkeit. Sie dient zur Bestimmung der in Pepsin-Salzsäure und Cellulase lösbaren organischen Substanz in Futtermitteln (Methodenbuch III Kapitel 6.6.1, VDLUFA, 1997).

\subsubsection{Zeitreihenversuch}

Die erste Beerntung fand 3 Wochen nach Vegetationsbeginn statt. Der Tag der Ernte richtete sich nach den Wetterverhältnissen, da die Ernte bei Sonnenschein erfolgen sollte. Im Versuchsjahr 2006 wurden einmal wöchentlich 2 Ernten jeweils morgens (8:00 Uhr) und mittags (13:00 Uhr) durchgeführt. Dies erfolgte bis zum Ende der Blüte, insgesamt wurden 4 Ernten durchgeführt (Tab. 8). Vegetationsbeginn des Versuchsjahrs 2006 war am 13.04.

Im Versuchsjahr 2007 erfolgte die Beerntung zweimal wöchentlich gegen 11:00 Uhr. Dies erfolgte zum Ende der Blüte plus 2 Ernten mehr, insgesamt wurden 11 Ernten durchgeführt (Tab. 8). Vegetationsbeginn des Versuchsjahrs 2007 war am 01.03. Wegen der Wetterverhältnisse fand die erste Beerntung 6 Wochen nach Vegetationsbeginn statt.

Wie genannt wurden die Proben nur im Verlauf des ersten Aufwuchses der Versuchsjahre 2006 und 2007 genommen. Die folgenden Schnitte galten als Pflegeschnitte. 
Tab. 8: Erntetermine im Zeitreiheversuch in beiden Versuchsjahren 2006 und 2007.

\begin{tabular}{|c|c|c|c|}
\hline \multicolumn{2}{|c|}{2006} & \multicolumn{2}{c|}{$\mathbf{2 0 0 7}$} \\
\hline Ernte & Datum & Ernte & Datum \\
\hline 1. Ernte & 03.05 & 1. Ernte & 12.04 \\
\hline 2. Ernte & 10.05 & 2. Ernte & 16.04 \\
\hline 3. Ernte & 17.05 & 3. Ernte & 19.04 \\
\hline 4. Ernte & 24.05 & 4. Ernte & 25.04 \\
\hline \multirow{5}{*}{} & & 5. Ernte & 28.04 \\
\cline { 3 - 4 } & & 6. Ernte & 02.05 \\
\cline { 3 - 4 } & & 7. Ernte & 06.05 \\
\cline { 3 - 4 } & & 8. Ernte & 13.05 \\
\cline { 3 - 4 } & & 9. Ernte & 16.05 \\
\cline { 3 - 4 } & & 10. Ernte & 24.05 \\
\cline { 3 - 4 } & & 11. Ernte & 30.05 \\
\hline
\end{tabular}

Bei jeder Ernte wurden die Phänologischen Stadien (BBCH) bestimmt und die Temperatur durch Temperaturmesser (digitale Thermometer) notiert und gemessen.

Die Proben wurden bei jeder Ernte aus einem neuen Stück der Parzelle bei einer Schnitthöhe von ca. 5 cm mit elektronischen Rasenkantenscheren Accu 6 (Firma Gardena) genommen, in einer Kühlbox mit Kühlelementen aufbewahrt und ins Institut gebracht.

Anschließend wurde eine Teilprobe für die Gesamttrockenmasse gehäckselt. Der andere Teil wurde in Blatt, Stängel und später auch in Ähren getrennt.

Im Versuchsjahr 2006 wurde ein Teil der separierten Proben auf flüssigem Stickstoff gemörsert und sofort bei $-25{ }^{\circ} \mathrm{C}$ gefroren. Der andere Teil wurde gefriergetrocknet.

Im Versuchsjahr 2007 wurden die separierten Proben nur gefriergetrocknet.

Die mit flüssigem Stickstoff behandelten Proben kamen direkt ins Labor zur chemischen Analysen. Das gefriergetrocknete Material wurde für die chemischen Analysen auf $1 \mathrm{~mm}$ mit der Brabendermühle vermahlen und ins Labor gegeben.

Im Labor wurden bei den mit flüssigem Stickstoff behandelten und gefriergetrockneten Proben die WlK-Gehalte und Fruktan mit HPLC bestimmt und zwar sowohl die Gesamtprobe, als auch die in Stängel, Blatt und im Versuchsjahr (2007) auch Ähre, separierten Proben.

Bei den gefriergetrockneten Proben wurde zusätzlich der Polymerisationsgrad des Fruktan mit HPLC bestimmt. 


\subsection{Zuckerbestimmung mittels HPLC}

1. Bestimmung von Fruktan, Saccharose, Glucose und Fructose mittels HPLC

1,0000 g Probe $\left(60{ }^{\circ} \mathrm{C}\right.$ oder gefriergetrocknet und auf $1 \mathrm{~mm}$ vermahlen) wird in einem $100 \mathrm{ml}$ Messkolben eingewogen (Doppelbestimmung).

Es werden ca. $70 \mathrm{ml}$ voll entsalztes Wasser $\left(80{ }^{\circ} \mathrm{C}\right)$ dazugegeben und bei dieser Temperatur 30 min in einem Wasserbad leicht bewegt.

Nach dem Abkühlen wird der Kolben bei $20{ }^{\circ} \mathrm{C}$ mit Wasser aufgefüllt und durch einen Faltenfilter Firma Schleicher und Schüll 595 1⁄2 filtriert.

$1 \mathrm{ml}$ des Extraktes wird in eine Klarglasflasche gefüllt und mittels HPLC analysiert.

Chromatrographische Bedingungen:

> Trennsäule: Rezex RPM Monosaccharide Firma Phenomenex

$>$ Ofentemperatur $80{ }^{\circ} \mathrm{C}$

$>$ Flow: 0,7 $\mathrm{ml} \mathrm{min}^{-1}$ voll entsalztes Wasser

$>$ RI - Detektor Shodex RI-71

$>$ Injektionsvolumen: $20 \mu \mathrm{l}$

$>$ Autosampler 460, Ofen Controler 480, Pumpe 420

$>$ Firma Kontron Instuments

$>$ Analysendauer $25 \mathrm{~min}$

\section{Auswertung mit Hilfe von externen Standards (Lineare Regression)}

\section{Standard1:}

je 20 mg Fruktan (Orafti Germany/Mannheim - Beneo ${ }^{\text {TM }}$ HP (Speicherkohlenhydrat aus der Zichorie))

Saccharose (Fluka)

Glucose (Fluka)

Fructose (Fluka)

in $100 \mathrm{ml}$ vollentsalztes Wasser lösen $=2 \%$

\section{Standard2:}

je $100 \mathrm{mg}$ der oben genannten Substanzen in $100 \mathrm{ml}$ voll entsalztem Wasser lösen $=10 \%$. 


\subsection{Bestimmung des Polymerisationsgrad von Fruktan}

Für die Analyse der Kettenlänge wurde das Probenmaterial aus dem Versuchsansatz der ,Zeitreihe‘ verwendet. Es wurden die fünf Sorten in elf aufeinanderfolgenden Terminen untersucht. Zum einen wurde eine Analyse aus dem oberirdischen Aufwuchs (Gesamtprobe) und zum anderen wurde aus einer getrennten Probe in Blatt und Stängel fraktioniertes Material untersucht. Die Analyse erfolgte aus gefriergetrocknetem Material in Anlehnung an eine Methode von Pavis et al. (2001).

Aufbereitung:

Die Proben wurden in einer wässrigen Lösung 30min in einem Schüttelwasserbad bei $80^{\circ} \mathrm{C}$ extrahiert. Anschließend durch einen Faltenfilter (Sartorius FT 4303185) gefiltert und in einer HPLC analysiert. Es wurde eine Trennsäule: Carbo Pac PA-100(4 x 250mm Analytical), Vorsäule Carbo Pac PA-100(4 x 50mm) von der Firma Dionex verwendet. Das Laufmittel für Eluent A war NaoH und Eluent B Natriumacetat. Detektiert wurde mit einem BioQuant PAM 2-MU Eletrochem.Detekor im Pulse Mode. Die Temperatur des Säulenofens betrug $25^{\circ} \mathrm{C}$. Die Auswertung erfolgte über zwei Inulin- bzw. Oligofructose-Standards (Raftiline ${ }^{\circledR}$; a. Hochpolymer, Beneo HR und b. Niederpolymer, Beneo GR; Firma Palatinit GmbH, Mannheim).

Entsprechend der Laufzeit wurden den einzelnen Peaks im Abgleich mit den Standards ein Polymerisationsgrad zugeordnet. Die Höhe des Peaks entspricht nicht einer Konzentration von Fruktanen in der Trockenmasse, sondern listet das Verhältnis der einzelnen Kettenlängen zueinander in der analysierten Probe auf. Aus Gründen der Übersichtlichkeit wurde der Polymerisationsgrad in Gruppen von jeweils 10 Peaks zusammengefasst, beginnend ab dem dritten Peak des Chromatogramms, d.h. die Peaks von 1 bis 10 entsprechen den Peaks 3 bis 12. Die ersten Peaks des Chromatogramms beziehen sich auf Einfachzucker (Glukose, Fruktose). 


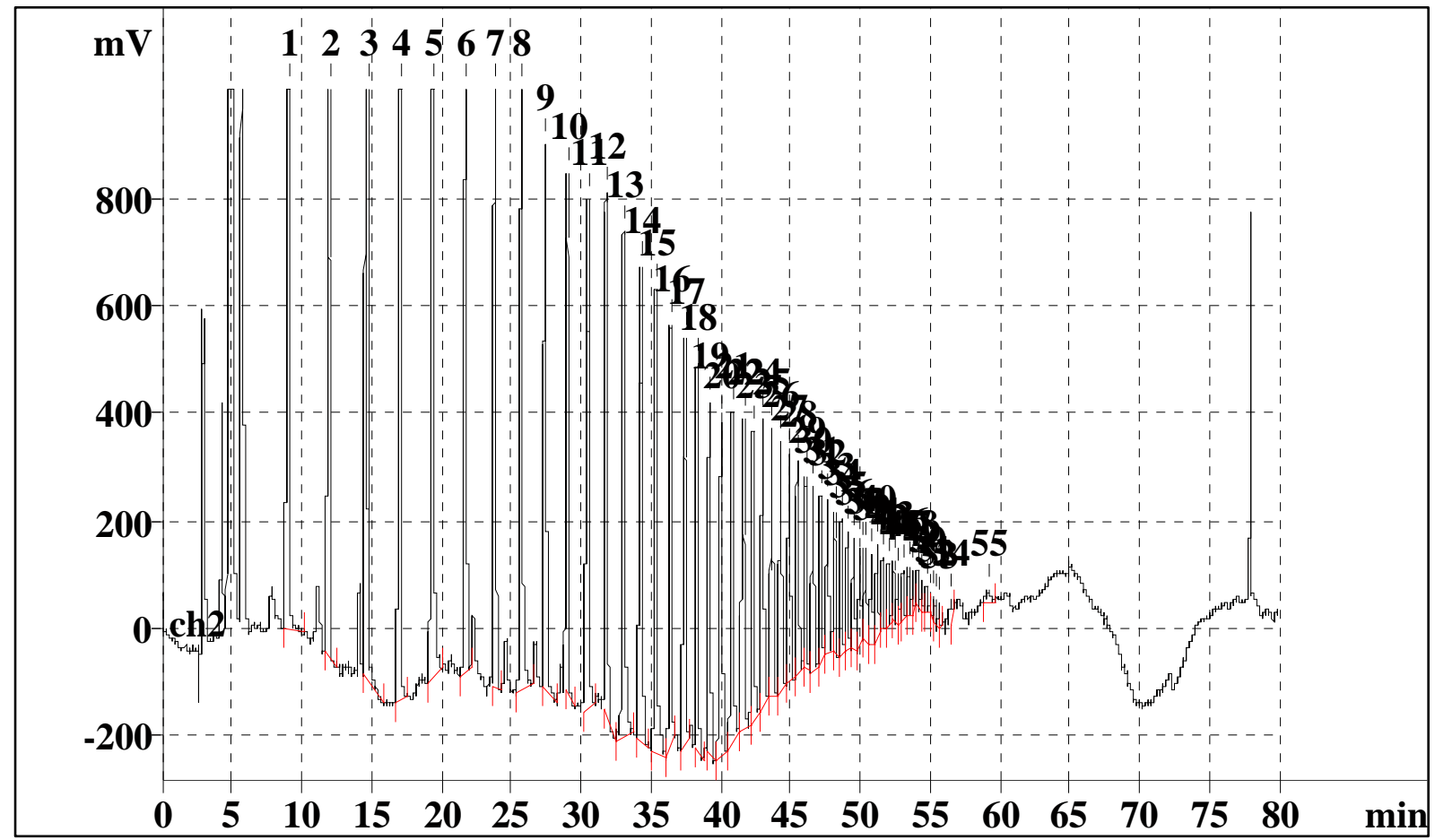

Abb. 7: Chromatogramm eines Gemisches von Beneo GR und Beneo HP im Verhältnis 1:1.

\subsection{Statistische Auswertung}

Die statistische Auswertung erfolgte mit dem Statistikprogramm „Statistical Analysis Systems“ Version 9.1.2 (SAS, 2004). Die Daten wurden vor der Verrechnung auf Normalverteilung geprüft. In den Ergebnisdarstellungen sind signifikante Unterschiede zwischen den Mittelwerten bei einer Irrtumswahrscheinlichkeit von $\mathrm{P}<0,05$ dargestellt. In der Ergebnisdarstellung werden die Signifikanzen in Form von Buchstaben angegeben, Werte mit gleichen Buchstaben sind nicht signifikant.

Für die Varianzanalyse wurde die GLM Prozedur benutzt, um die Vergleiche innerhalb der Varianten durchzuführen.

Die Vergleiche (Versuchsjahr-, Schnittzeitpunkt-, Versuchsstandort-, Tageszeiten- und NDüngungsstufenvergleich) wurden mit Paired-Samples T-Test durchgeführt.

Die Beziehungen zwischen den verschiedenen Parametern wurden mit Bivariate Correlations durchgeführt. 


\section{Ergebnisse}

\subsection{Ergebnisse Sortenversuch}

\subsubsection{Trockenmasseerträge}

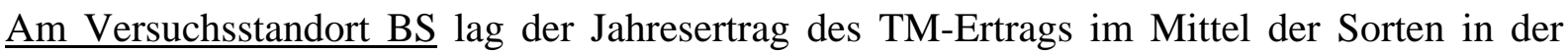
Frühschnittvariante (FSV) im 1HVJ (2006) mit 111 dt/ha deutlich über dem Ergebnis des $2 H V J$ (2007) mit 95 dt/ha und in der Spätschnittvariante (SSV) im 1HVJ mit 138 dt/ha auch deutlich über dem Ergebnis des 2HVJ mit 119 dt/ha (Tab. 9). Ebenso lag der TM-Ertrag im 1. Schnitt in der FSV und in der SSV im 1HVJ deutlich über den TM-Ertrag des 2HVJ.

Am Versuchsstandort HS, dagegen lag der Jahresertrag des TM-Ertrags im Mittel der Sorten in der FSV im 1HVJ mit $134 \mathrm{dt} /$ ha deutlich unter dem Ergebnis des $2 \mathrm{HVJ}$ mit $142 \mathrm{dt} / \mathrm{ha}$ und in der SSV im 1HVJ mit 155 dt/ha auch deutlich unter dem Ergebnis des 2HVJ mit 170 dt/ha (Tab. 9). Im 1. Schnitt wies der TM-Ertrag in der FSV keinen signifikanten Unterschied zwischen 1HVJ und 2HVJ auf. In der SSV lag der TM-Ertrag im 1HVJ unter dem TM-Ertrag des $2 \mathrm{HVJ}$.

Im Mittel der beiden Versuchsstandorten (BS und HS) wies der Jahresertrag des TM-Ertrags im Mittel der Sorten in der FSV keinen signifikanten Unterschied zwischen 1HVJ (122,4 dt/ha) und 2HVJ (118,6 dt/ha) auf. Auch wies der Jahresertrag des TM-Ertrags im Mittel der Sorten in der SSV keinen signifikanten Unterschied zwischen 1HVJ (146,4 dt/ha) und 2HVJ $(144,6 \mathrm{dt} / \mathrm{ha})$ auf.

Tab. 9: Versuchsjahresvergleich (2006/2007) für TM-Ertrag dt/ha Mittelwert aus allen Sorten im 1. Schnitt und im Jahresertrag.

\begin{tabular}{|c|c|c|c|c|c|c|c|c|c|c|c|c|}
\hline \multicolumn{13}{|l|}{ 1. Schnitt } \\
\hline \multirow{3}{*}{ Versuchsjahr } & \multicolumn{6}{|c|}{ BS } & \multicolumn{6}{|c|}{ HS } \\
\hline & \multicolumn{3}{|c|}{ FSV } & \multicolumn{3}{|c|}{ SSV } & \multicolumn{3}{|c|}{ FSV } & \multicolumn{3}{|c|}{ SSV } \\
\hline & Mw & & SD & $\mathrm{Mw}$ & & SD & Mw & & SD & Mw & & SD \\
\hline 2006 & 49 & $\mathrm{a}$ & 9,1 & 78 & $\mathrm{a}$ & 10,8 & 52 & $\mathrm{a}$ & 6,5 & 66 & $\mathrm{~b}$ & 6,2 \\
\hline 2007 & 29 & $\mathrm{~b}$ & 7,7 & 58 & $\mathrm{~b}$ & 12,5 & 54 & $\mathrm{a}$ & 10,4 & 73 & $\mathrm{a}$ & 9,8 \\
\hline \multicolumn{13}{|l|}{ Jahresertrag } \\
\hline 2006 & 111 & $\mathrm{a}$ & 9,8 & 138 & $\mathrm{a}$ & 11,2 & 134 & $\mathrm{~b}$ & 11,5 & 155 & $\mathrm{~b}$ & 10,7 \\
\hline 2007 & 95 & $\mathrm{~b}$ & 16,7 & 119 & $\mathrm{~b}$ & 18,1 & 142 & $\mathrm{a}$ & 12,8 & 170 & $\mathrm{a}$ & 11,4 \\
\hline
\end{tabular}

Signifikante Unterschiede gelten für Werte in derselben Spalte mit unterschiedlichen Buchstaben bei $\mathrm{P}<0,05$, SD = Standardabweichung, Mw = Mittelwert aus allen 20 Sorten und 3 Wiederholungen .

Die Sorte Lidaglo (Knaulgras) hatte auf beiden Versuchsstandorten (BS und HS), bei beiden Schnittzeitpunktvarianten (FSV und SSV) und in beiden HVJ die höchsten TM-Erträge wie die Abbildungen 8 bis 15 verdeutlichen. 
Die Aufteilung des TM-Jahresertrages in die einzelnen Schnitte zeigte, dass der 1. Schnitt den Hauptbeitrag zum gesamten TM-Ertrag der HVJ an den beiden Versuchsstandorten und bei beiden Schnittzeitpunktvarianten (FSV und SSV) lieferte (Abb. 8 bis 15). Der prozentuale Anteil des 1. Schnittes am Jahres-TM-Ertrag wurde in der Tabelle A1 im Anhang dargestellt.

Sowohl im 1. Schnitt als auch im Jahresertrag waren die Trockenmasseerträge der SSV an beiden Versuchsstandorten (BS und HS) höher als die Trockenmasseerträge der FSV (Tab. 10).

Tab. 10: Schnittzeitpunktvergleich (FSV und SSV) für TM-Ertrag (dt/ha) Mittelwert aus allen Sorten und Versuchsjahren (2006 und 2007) im 1. Schnitt und im Jahresertrag.

\begin{tabular}{|c|c|c|c|c|c|c|c|c|c|c|c|c|}
\hline \multirow{3}{*}{ Schnittzeitpunkt } & \multicolumn{6}{|c|}{ BS } & \multicolumn{6}{|c|}{ HS } \\
\hline & \multicolumn{3}{|c|}{ 1. Schnitt } & \multicolumn{3}{|c|}{ Jahresertrag } & \multicolumn{3}{|c|}{ 1. Schnitt } & \multicolumn{3}{|c|}{ Jahresertrag } \\
\hline & Mw & & SD & Mw & & SD & $\mathrm{Mw}$ & & SD & $\mathrm{Mw}$ & & SD \\
\hline FSV & 39 & $\mathrm{~b}$ & 12,8 & 103 & $\mathrm{~b}$ & 15,8 & 53 & $\mathrm{~b}$ & 8,7 & 138 & $\mathrm{~b}$ & 12,8 \\
\hline SSV & 68 & $\mathrm{a}$ & 15,6 & 129 & $\mathrm{a}$ & 17,6 & 70 & $\mathrm{a}$ & 8,8 & 162 & $\mathrm{a}$ & 13,3 \\
\hline
\end{tabular}

Signifikante Unterschiede gelten für Werte in derselben Spalten mit unterschiedlichen Buchstaben bei $\mathrm{P}<0,05$, $\mathrm{SD}=$ Standardabweichung, $\mathrm{Mw}=$ Mittelwert aus allen 20 Sorten, beiden Versuchsjahre $(1 \mathrm{HVJ}$ und $2 \mathrm{HVj})$ und 3 Wiederholungen. 


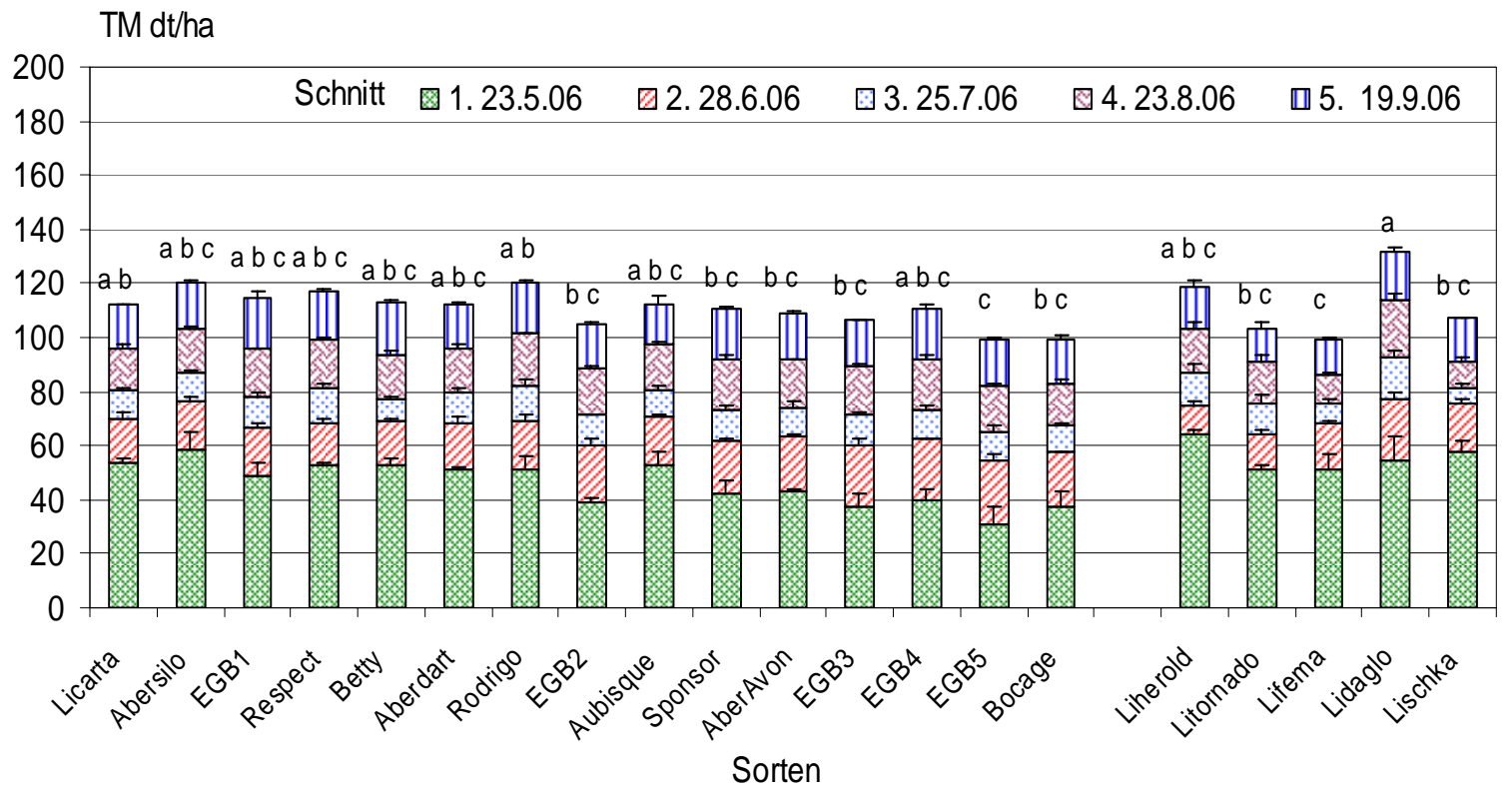

Abb. 8: TM-Ertrag dt/ha für fünf Schnitte der FSV des 1HVJ an Versuchsstandort (BS), signifikante Unterschiede gelten für Säulen mit unterschiedlichen Buchstaben $(\mathrm{P}<0,05)$, Fehlerbalken kennzeichnen die Standardabweichung.

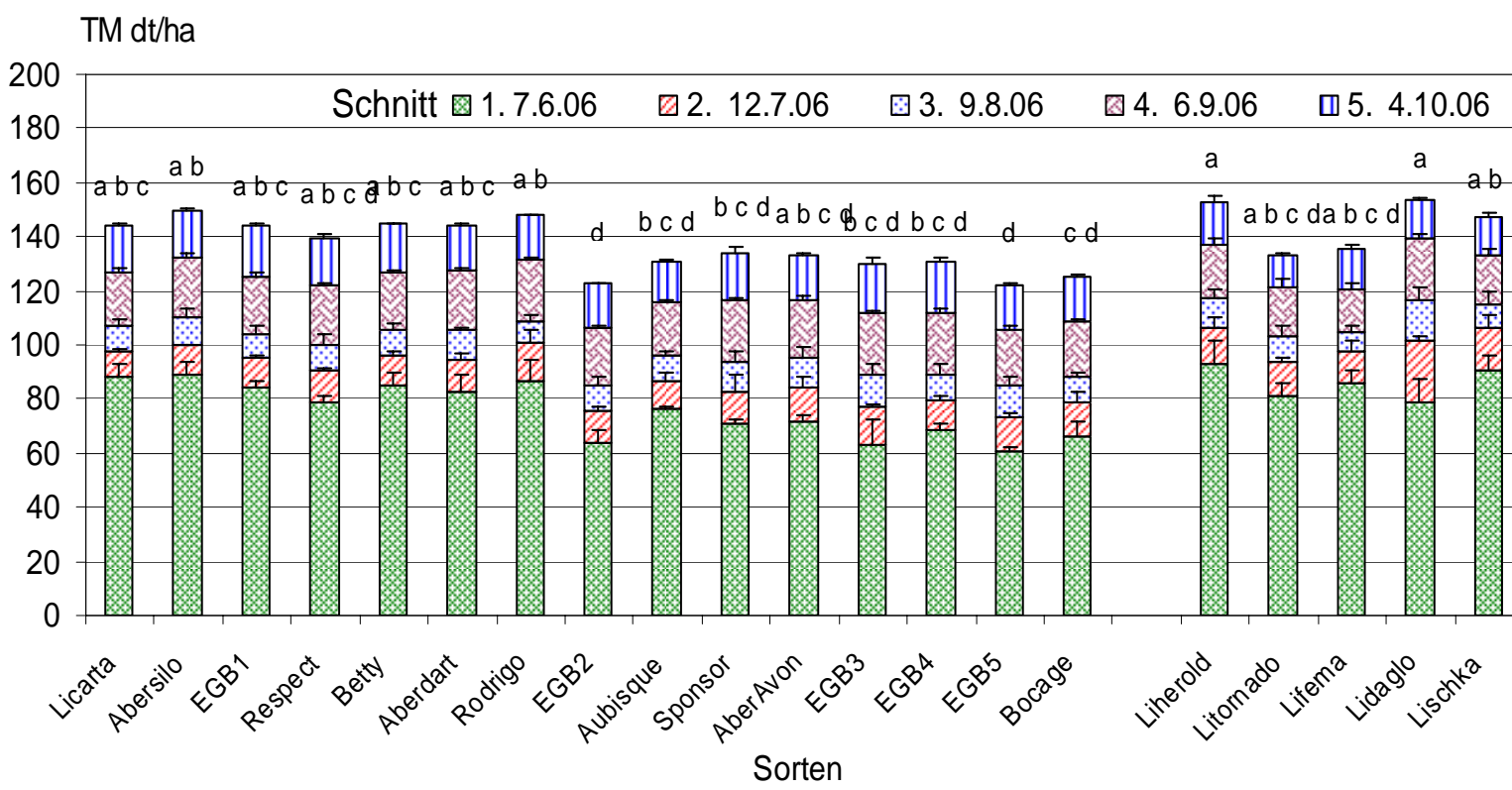

Abb. 9: TM-Ertrag dt/ha der für fünf Schnitte der SSV des 1HVJ an Versuchsstandort (BS), signifikante Unterschiede gelten für Säulen mit unterschiedlichen Buchstaben $(\mathrm{P}<0,05)$, Fehlerbalken kennzeichnen die Standardabweichung. 


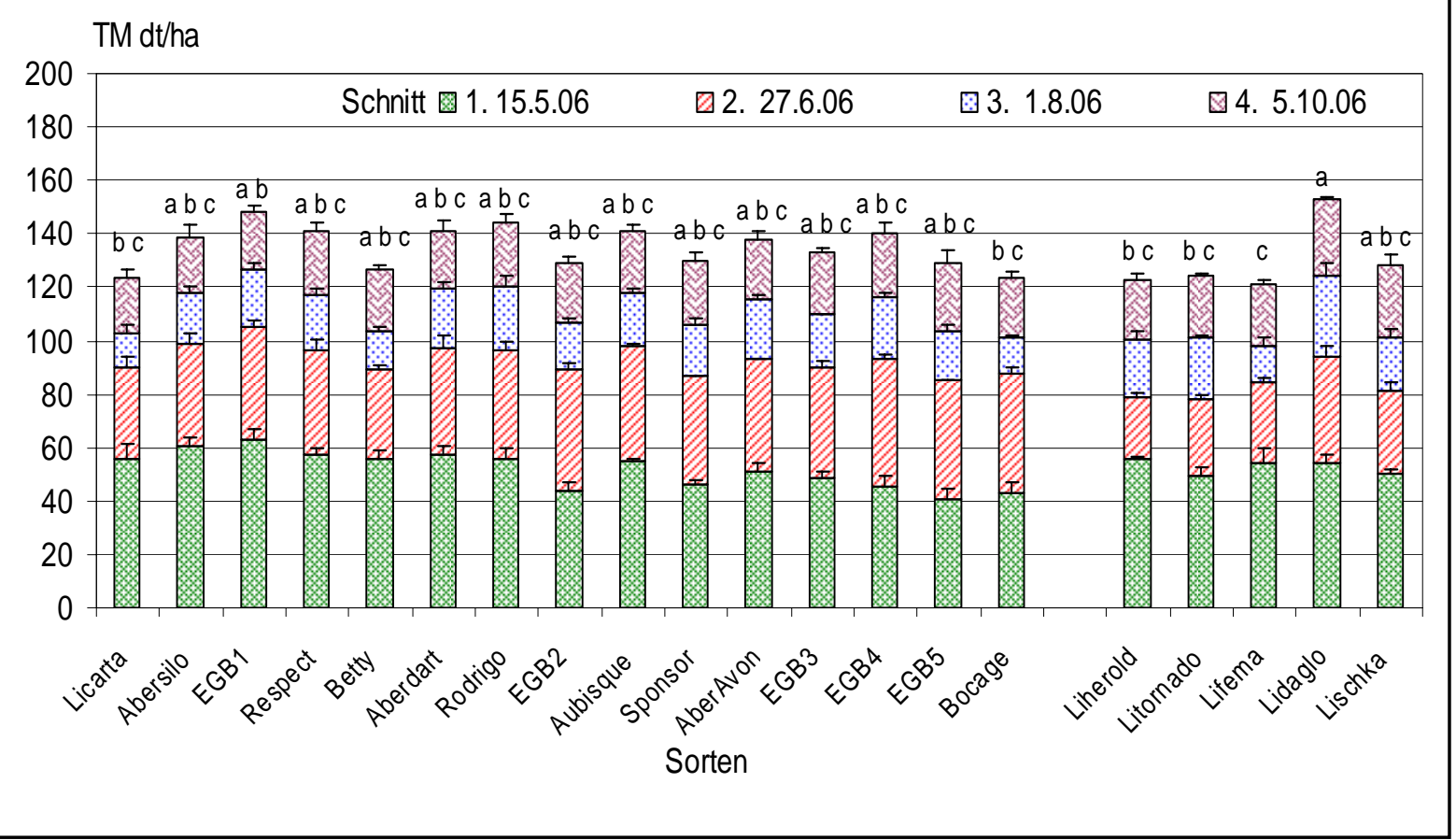

Abb. 10: TM-Ertrag dt/ha für vier Schnitte der FSV des 1HVJ an Versuchsstandort (HS), signifikante Unterschiede gelten für Säulen mit unterschiedlichen Buchstaben $(\mathrm{P}<0,05)$, Fehlerbalken kennzeichnen die Standardabweichung.

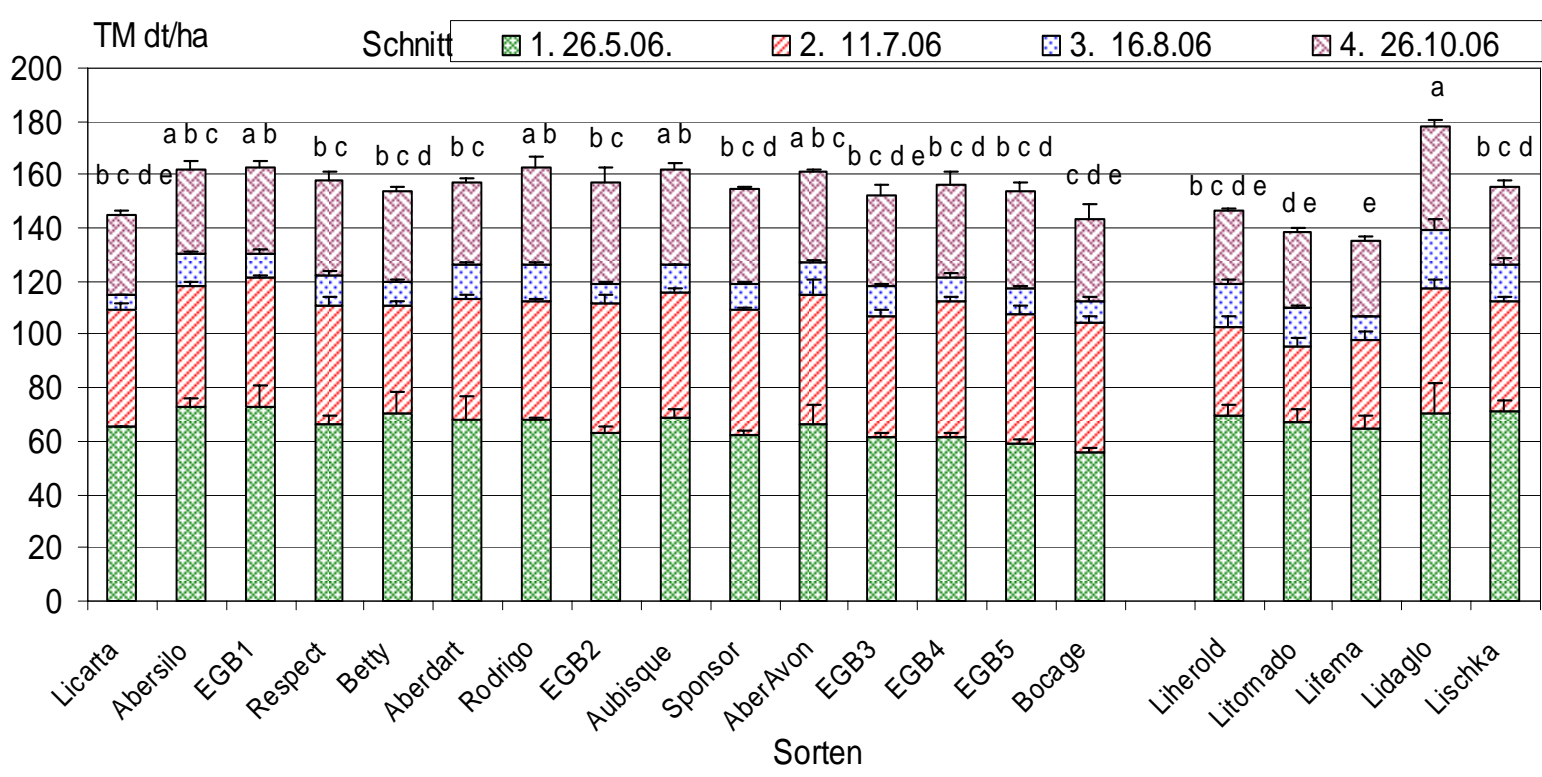

Abb. 11: TM-Ertrag dt/ha für vier Schnitte der SSV des 1HVJ an Versuchsstandort (HS), signifikante Unterschiede gelten für Säulen mit unterschiedlichen Buchstaben $(\mathrm{P}<0,05)$, Fehlerbalken kennzeichnen die Standardabweichung. 


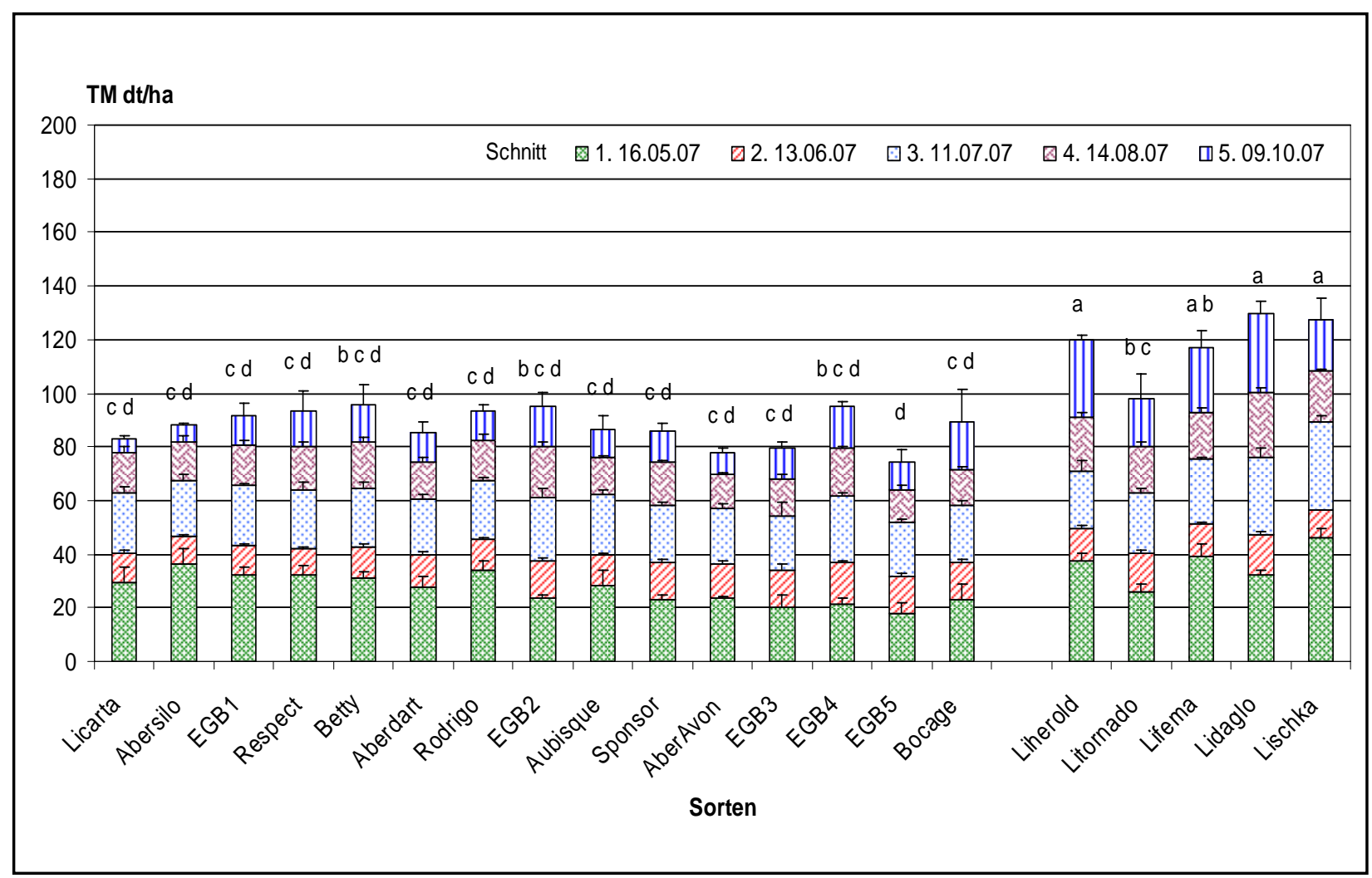

Abb. 12: TM-Ertrag dt/ha für fünf Schnitte der FSV des 2HVJ an Versuchsstandort (BS), signifikante Unterschiede gelten für Säulen mit unterschiedlichen Buchstaben $(\mathrm{P}<0,05)$, Fehlerbalken kennzeichnen die Standardabweichung.

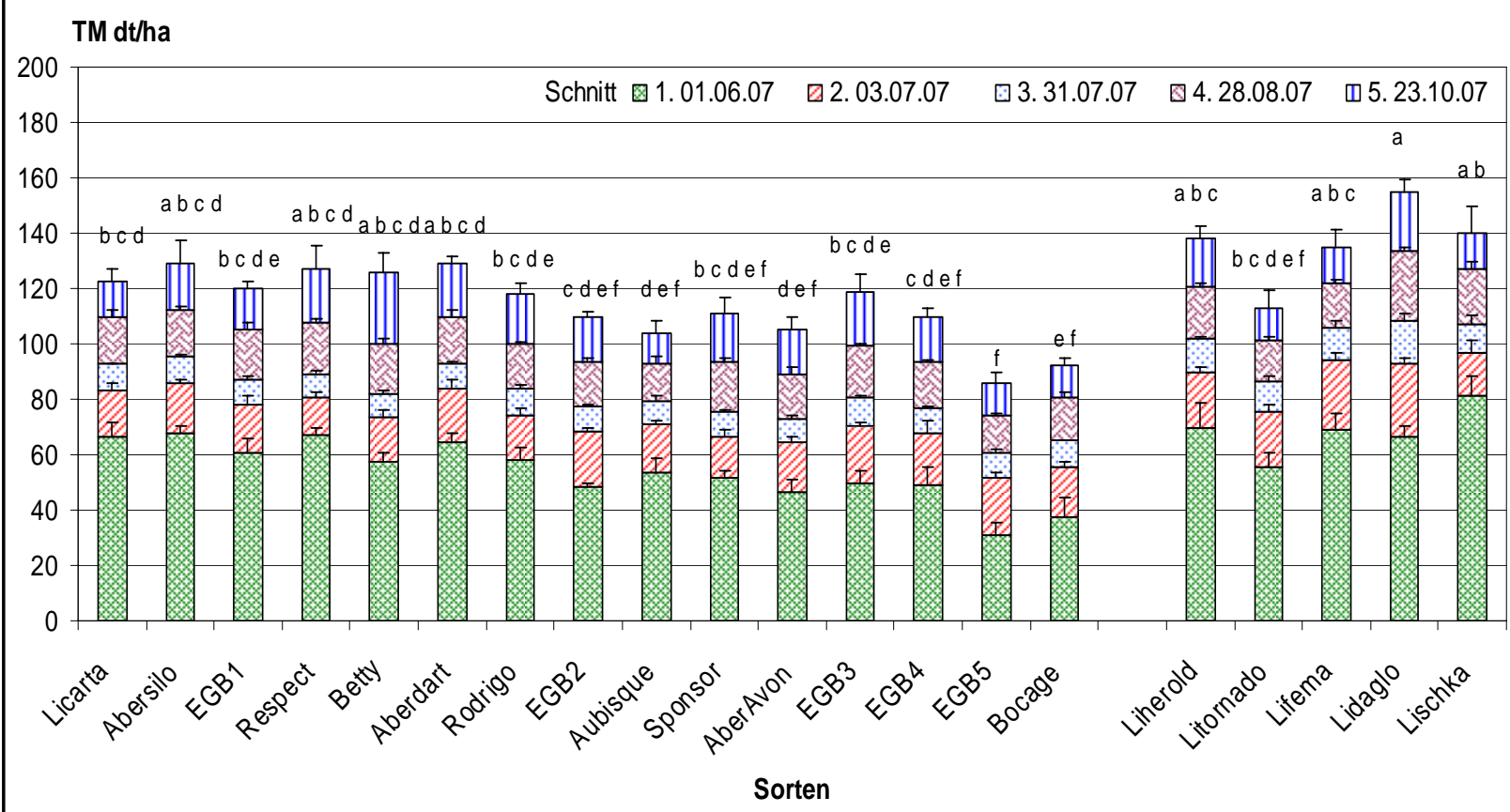

Abb. 13: TM-Ertrag dt/ha für fünf Schnitte der SSV des 2HVJ an Versuchsstandort (BS), signifikante Unterschiede gelten für Säulen mit unterschiedlichen Buchstaben $(\mathrm{P}<0,05)$, Fehlerbalken kennzeichnen die Standardabweichung. 


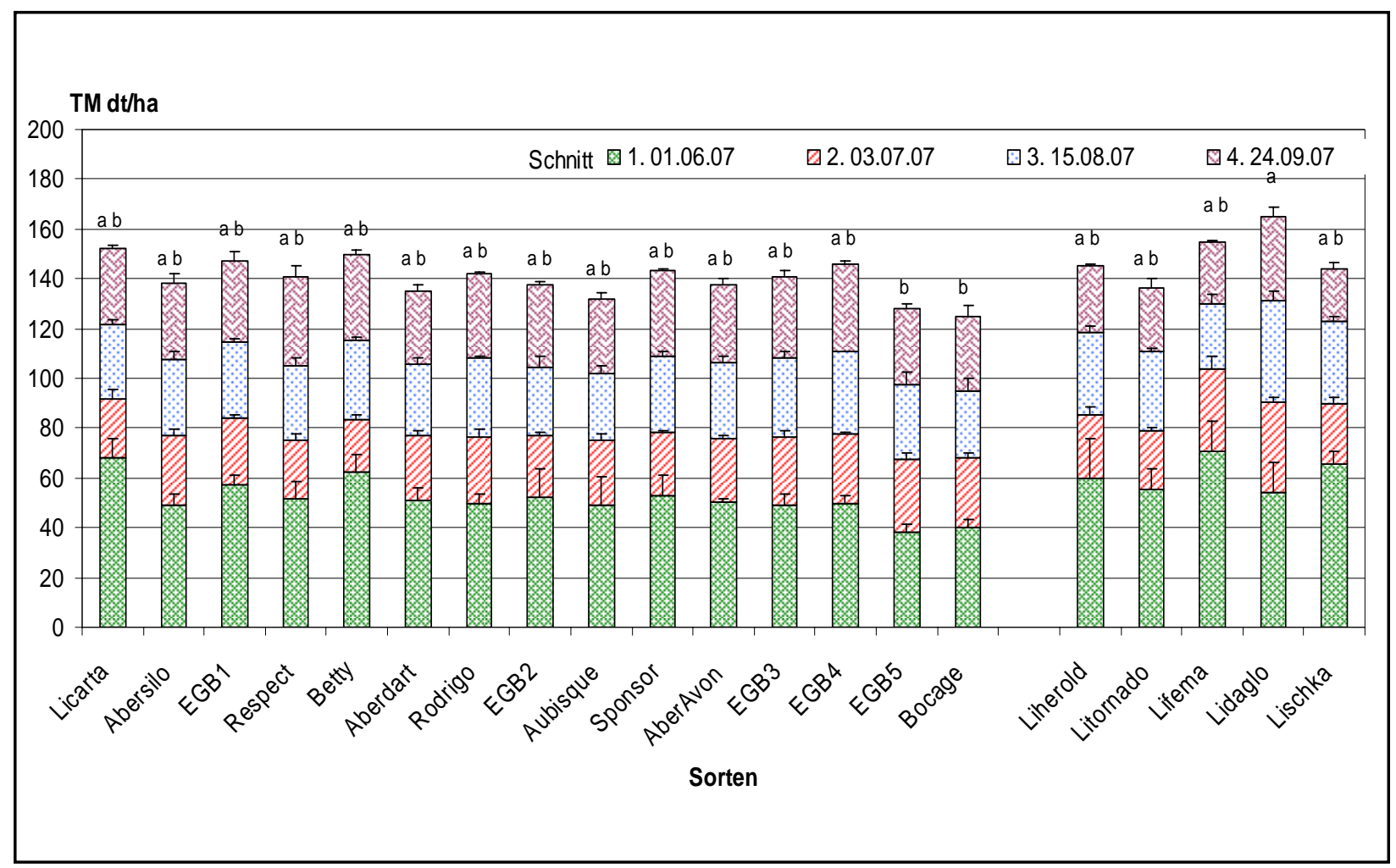

Abb. 14: TM-Ertrag dt/ha für vier Schnitte der FSV des 2HVJ an Versuchsstandort (HS), signifikante Unterschiede gelten für Säulen mit unterschiedlichen Buchstaben $(\mathrm{P}<0,05)$, Fehlerbalken kennzeichnen die Standardabweichung.

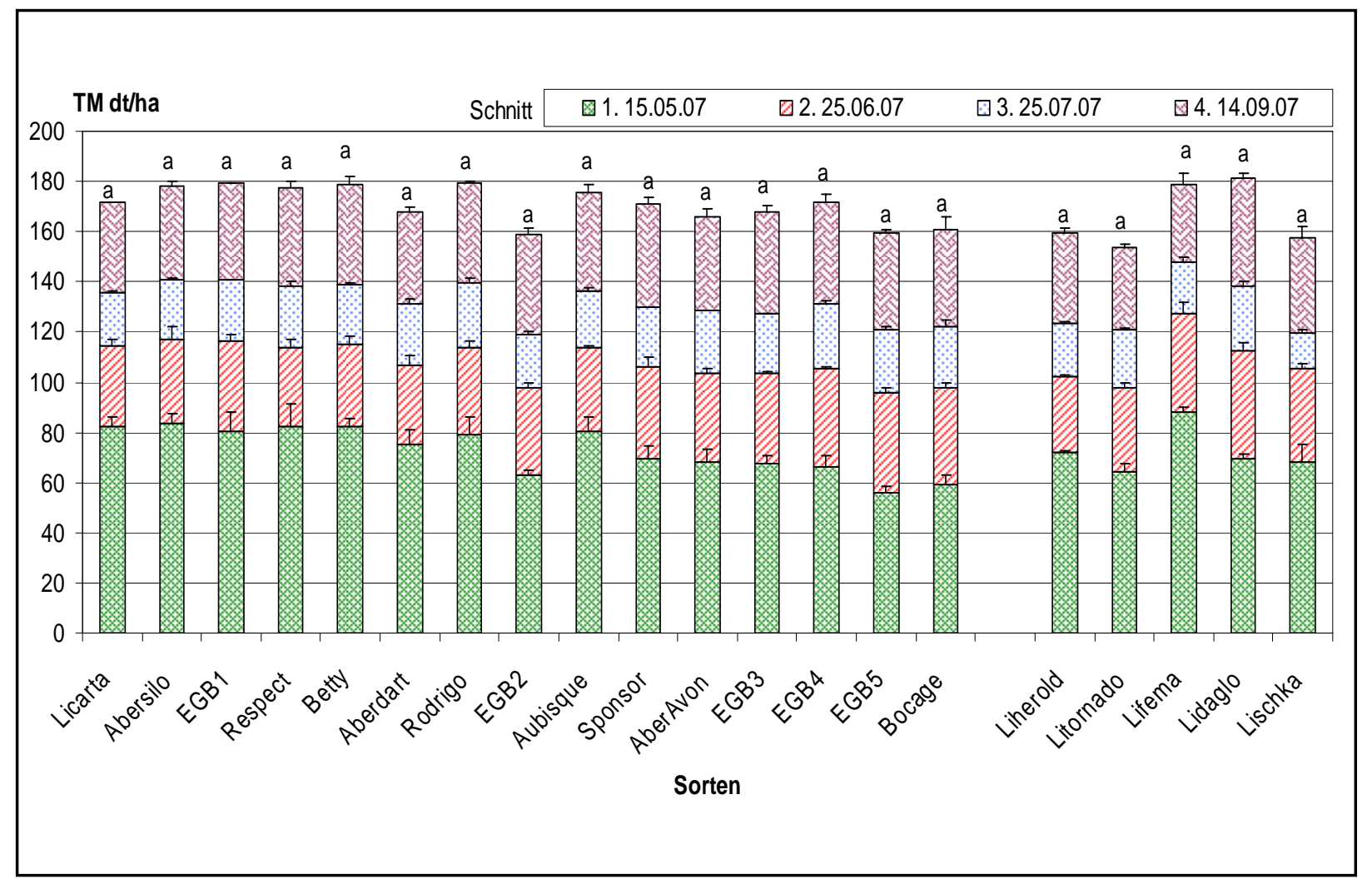

Abb. 15: TM-Ertrag dt/ha für vier Schnitte der SSV des 2HVJ an Versuchsstandort (HS), signifikante Unterschiede gelten für Säulen mit unterschiedlichen Buchstaben $(\mathrm{P}<0,05)$, Fehlerbalken kennzeichnen die Standardabweichung. 
Der TM-Ertrag in der FSV war am Versuchsstandort HS in beiden HVJ höher als am Versuchsstandort BS. Der TM-Ertrag in der SSV war am Versuchsstandort BS im 1HVJ höher als am Versuchsstandort HS. Dagegen war der TM-Ertrag der SSV am Versuchsstandort HS im 2HVJ höher als am Versuchsstandort BS (Tab. 11).

Im Mittel der beiden HVJ war der TM-Ertrag im 1. Schnitt der FSV am Versuchsstandort HS (53 dt/ha) höher als am Versuchsstandort BS (38,9 dt/ha). In der SSV im Mittel der beiden HVJ wies der TM-Ertrag keinen signifikanten Unterschied zwischen den Versuchsstandorten (BS und HS) auf.

Tab. 11: TM-Erträge (dt/ha) an beiden Versuchsstandorten (BS und HS) im 1. Schnitt, Mittelwert aus allen Sorten.

\begin{tabular}{|c|c|c|c|c|c|c|c|c|c|c|c|c|}
\hline \multirow{3}{*}{ Standort } & \multicolumn{6}{|c|}{2006} & \multicolumn{6}{|c|}{2007} \\
\hline & \multicolumn{3}{|c|}{ FSV } & \multicolumn{3}{|c|}{ SSV } & \multicolumn{3}{|c|}{ FSV } & \multicolumn{3}{|c|}{ SSV } \\
\hline & $\mathrm{Mw}$ & & $\mathrm{SD}$ & $\mathrm{Mw}$ & & $\mathrm{SD}$ & $\mathrm{Mw}$ & & $\mathrm{SD}$ & Mw & & SD \\
\hline BS & 49 & $b$ & 9,2 & 78 & $\mathrm{a}$ & 10,8 & 29 & $\mathrm{~b}$ & 7,7 & 58 & $b$ & 12,6 \\
\hline HS & 52 & $\mathrm{a}$ & 6,6 & 66 & $\mathrm{~b}$ & 6,2 & 54 & $\mathrm{a}$ & 10,4 & 73 & $\mathrm{a}$ & 9,8 \\
\hline
\end{tabular}

Signifikante Unterschiede gelten für Werte in derselben Spalte mit unterschiedlichen Buchstaben bei $\mathrm{P}<0,05$, $\mathrm{SD}=$ Standardabweichung, $\mathrm{Mw}=$ Mittelwert aus allen 20 Sorten und 3 Wiederholungen .

Tabellen 12 und 13 zeigen die Unterschiede zwischen den Sorten in den Reifegruppen früh, mittel und spät im 1. und 2.HVJ.

Reifegruppe „früh“:

Am Versuchsstandort BS im 1. und 2.HVJ wies der TM-Ertrag zu den Schnittzeitpunkten (FSV und SSV) im 1. Schnitt und im Jahresertrag keine Unterschiede zwischen den Sorten (Licarta, Abersilo und EGB1) in der Reifegruppe „früh“ auf (Tab. 12).

Gleiches gilt für den Versuchsstandort HS, allerdings mit zwei Ausnahmen im 1HVJ im Jahressertrag der SSV, wo der TM-Ertrag der Sorte EGB1 mit 163 dt/ha deutlich höher als der TM-Ertrag der Sorte Licarta (145 dt/ha) lag und im 2HVJ im 1. Schnitt der FSV, wo der TMErtrag der Sorte Licarta mit 68 dt/ha deutlich höher als der TM-Ertrag der Sorte Abersilo (49 dt/ha) lag (Tab. 13).

Im Mittel der beiden Versuchsjahre (1HVJ und 2HVJ) und der beiden Versuchsstandorten (BS und HS) wies der TM-Ertrag zu den Schnittzeitpunkten (FSV und SSV) im Jahresertrag keine signifikanten Unterschiede zwischen den Sorten (Licarta, Abersilo und EGB1) in der Reifegruppe „früh“ auf (Tab. 14). 


\section{Reifegruppe „mittel“:}

Im 1HVJ wies der TM-Ertrag in BS in der FSV im 1. Schnitt signifikante Unterschiede zwischen der Sorte EGB2 und den Sorten Respect, Betty, Aberdart, Rodrigo und Aubisque auf. Erster hatte einen niedrigeren TM-Ertrag als die übrigen Sorten (Tab. 12). Im Jahresertrag gab es signifikante Unterschiede nur zwischen der Sorte Rodrigo (121 dt/ha) und der Sorte EGB2 (105 dt/ha). In der SSV im 1. Schnitt traten signifikante Unterschiede zwischen der Sorte EGB2 und den Sorten Respect, Betty, Aberdart und Rodrigo auf. Der TM-Ertrag der Sorte EGB2 war signifikant niedriger (Tab. 12). Im Jahresertrag gab es signifikante Unterschiede zwischen den Sorten, die Sorte Rodrigo hatte den höchsten TM-Ertrag (48 dt/ha), die Sorte EGB2 hatte den niedrigsten TM-Ertrag (123 dt/ha).

Im 2HVJ und in der FSV wies der TM-Ertrag im 1. Schnitt signifikante Unterschiede nur zwischen der Sorte Rodrigo (34 dt/ha) und der Sorte EGB2 (23 dt/ha) auf. Im Jahresertrag gab es keine signifikanten Unterschiede zwischen den Sorten. In der SSV im 1. Schnitt gab es signifikante Unterschiede zwischen den Sorten; die Sorte Respect hatte den höchsten TMErtrag (67 dt/ha), die Sorte EGB2 hatte den niedrigsten TM-Ertrag (49 dt/ha). Im Jahresertrag zeigten sich keine Unterschiede zwischen den Sorten.

Sowohl im 1HVJ als auch im 2HVJ wies der TM-Ertrag am Versuchsstandort HS in den Schnittzeitpunkten (FSV und SSV) im 1. Schnitt und im Jahresertrag keine signifikanten Unterschiede zwischen den Sorten auf. Zwei Ausnahmen gab es im 1HVJ im 1. Schnitt der FSV. Der TM-Ertrag der Sorte EGB2 unterschied sich signifikant von den Sorten Respect, Betty, Aberdart, Rodrigo und Aubisque. Im 1. Schnitt der SSV wies der TM-Ertrag signifikante Unterschiede zwischen die Sorten; die Sorten Respect und Betty hatten den höchsten TM-Ertrag (82 dt/ha), die Sorte EGB2 hatte den niedrigsten TM-Ertrag (63 dt/ha) (Tab 13).

Im Mittel der beiden Versuchsjahre (1HVJ und 2HVJ) und der beiden Versuchsstandorten (BS und HS) wies der TM-Ertrag in der FSV im Jahresertrag keine signifikanten Unterschiede zwischen den Sorten (Respect, Betty, Aberdart, Rodrigo, EGB2 und Aubisque) in der Reifegruppe,,mittel“ auf. In der SSV wies der TM-Ertrag signifikante Unterschiede zwischen die Sorten; die Sorten Rodrigo hatte den höchsten TM-Ertrag (152 dt/ha), die Sorte EGB2 hatte den niedrigsten TM-Ertrag (137,3 dt/ha) (Tab. 14). 


\section{Reifegruppe „spät“:}

Am Versuchsstandort BS im 1HVJ wies der TM-Ertrag zu den Schnittzeitpunkten (FSV und SSV) im 1. Schnitt und im Jahresertrag keine Unterschiede zwischen den Sorten auf. Im 2HVJ gab es auch in der FSV im1. Schnitt und im Jahresertrag keine Unterschiede zwischen den Sorten. In der SSV im 1. Schnitt traten signifikante Unterschiede zwischen der Sorte EGB5 und den Sorten Sponsor, AberAvon, EGB3 und EGB4 auf. Im Jahresertrag gab es signifikante Unterschiede zwischen den Sorten; die Sorte EGB3 hatte den höchsten TMErtrag (119 dt/ha), die Sorte EGB5 hatte den niedrigsten TM-Ertrag (86 dt/ha) (Tab. 12).

Am Versuchsstandort HS zeigten sich in der im 1. Schnitt signifikanten Unterschiede in TMErtrag zwischen den Sorten im 1HVJ; Die Sorte AberAvon hatte den höchsten TM-Ertrag (51 dt/ha), die Sorte EGB5 hatte den niedrigsten TM-Ertrag (40 dt/ha). Im Jahresertrag gab es keine signifikanten Unterschiede zwischen den Sorten. In der SSV gab es im 1. Schnitt signifikante Unterschiede zwischen der Sorte AberAvon (66 dt/ha) und der Sorte Bocage (55 dt/ha). Auch im Jahresertrag gab es signifikante Unterschiede zwischen AberAvon (161 dt/ha) und (144 dt/ha). Im 2HVJ gab es in der FSV im 1. Schnitt signifikante Unterschiede zwischen den Sorten; die Sorte Sponsor hatte den höchsten TM-Ertrag (53 dt/ha), die Sorte EGB5 hatte den niedrigsten TM-Ertrag (38 dt/ha). Im Jahresertrag zeigten sich keine signifikanten Unterschiede zwischen den Sorten. In der SSV gab es im 1. Schnitt signifikante Unterschiede zwischen den Sorten; die Sorte Sponsor hatte den höchsten TM-Ertrag (70 dt/ha), die Sorte EGB5 hatte den niedrigsten TM-Ertrag (56 dt/ha). Im Jahresertrag zeigten sich keine signifikanten Unterschiede zwischen den Sorten (Tab. 13).

Im Mittel der beiden Versuchsjahre (1HVJ und 2HVJ) und der beiden Versuchsstandorten (BS und HS) wies der TM-Ertrag in der FSV im Jahresertrag signifikante Unterschiede zwischen den Sorten (Sponsor, AberAvon, EGB3, EGB4, EGB5 und Bocage) in der Reifegruppe,,spät“ auf; die Sorte EGB4 hatte den höchsten TM-Ertrag (123 dt/ha), die Sorte EGB5 hatte den niedrigsten TM-Ertrag (107,3 dt/ha). In der SSV wies der TM-Ertrag keine signifikanten Unterschiede zwischen den Sorten in der Reifegruppe,,spät“ auf (Tab 14). 
Tab. 12: TM-Ertrag (dt/ha) in Abhängigkeit von den Sortenreifegruppen (früh, mittel und spät) von Deutschen Weidelgras am Versuchsstandort BS im 1HVJ (2006) und im 2HVJ (2007).

\begin{tabular}{|c|c|c|c|c|c|c|c|c|c|}
\hline \multicolumn{10}{|c|}{ 1HVJ (2006) } \\
\hline \multirow{3}{*}{\multicolumn{2}{|c|}{$\begin{array}{l}\text { Reifegruppe/ } \\
\text { Sorten }\end{array}$}} & \multicolumn{8}{|c|}{ Schnittzeitpunkt } \\
\hline & & \multicolumn{4}{|c|}{ FSV } & \multicolumn{4}{|c|}{ SSV } \\
\hline & & $\begin{array}{l}1 . \\
\text { Schnitt }\end{array}$ & SD & Jahresertrag & SD & $\begin{array}{l}1 . \\
\text { Schnitt }\end{array}$ & SD & Jahresertrag & SD \\
\hline \multirow{3}{*}{ früh } & Licarta & $54 \mathrm{a}$ & 1,3 & $112 \mathrm{a}$ & 1,5 & $88 \mathrm{a}$ & 4,4 & $144 \mathrm{a}$ & 7,5 \\
\hline & Abersilo & $58 \mathrm{a}$ & 6,5 & $120 \mathrm{a}$ & 5,9 & 89 a & 4,8 & $150 \mathrm{a}$ & 9,5 \\
\hline & EGB1 & $49 \mathrm{a}$ & 4,5 & $114 \mathrm{a}$ & 8,6 & $84 \mathrm{a}$ & 2,7 & $144 \mathrm{a}$ & 5,0 \\
\hline \multirow{6}{*}{ mittel } & Respect & $53 \mathrm{a}$ & 1,4 & $117 \mathrm{a} b$ & 3,1 & $79 \mathrm{a}$ & 2,3 & $139 \mathrm{ab}$ & 6,2 \\
\hline & Betty & $53 \mathrm{a}$ & 2,7 & 113 a b & 1,6 & $85 \mathrm{a}$ & 4,8 & $145 \mathrm{a} b$ & 5,5 \\
\hline & Aberdart & $51 \mathrm{a}$ & 1,1 & $112 \mathrm{a} \mathrm{b}$ & 5,1 & $83 \mathrm{a}$ & 6,6 & $144 \mathrm{a} \mathrm{b}$ & 5,1 \\
\hline & Rodrigo & $51 \mathrm{a}$ & 4,9 & $121 \mathrm{a}$ & 4,2 & $86 \mathrm{a}$ & 8,0 & $148 \mathrm{a}$ & 7,0 \\
\hline & EGB2 & $39 \mathrm{~b}$ & 1,2 & $105 \mathrm{~b}$ & 0,4 & $64 \mathrm{~b}$ & 4,4 & $123 \mathrm{c}$ & 3,6 \\
\hline & Aubisque & $53 \mathrm{a}$ & 5,0 & $112 \mathrm{a} b$ & 9,9 & $76 \mathrm{a} \mathrm{b}$ & 1,2 & $131 \mathrm{~b} \mathrm{c}$ & 2,2 \\
\hline \multirow{6}{*}{ spät } & Sponsor & $42 \mathrm{a}$ & 4,7 & $110 \mathrm{a}$ & 4,7 & $71 \mathrm{a}$ & 1,8 & $130 \mathrm{a}$ & 10,0 \\
\hline & AberAvon & $43 \mathrm{a}$ & 0,9 & $109 \mathrm{a}$ & 2,3 & $72 \mathrm{a}$ & 2,7 & $133 \mathrm{a}$ & 6,6 \\
\hline & EGB3 & $37 \mathrm{a}$ & 5,2 & $106 \mathrm{a}$ & 7,9 & $63 \mathrm{a}$ & 9,6 & $130 \mathrm{a}$ & 12,0 \\
\hline & EGB4 & $39 \mathrm{a}$ & 4,2 & $111 \mathrm{a}$ & 7,5 & $68 \mathrm{a}$ & 2,3 & $130 \mathrm{a}$ & 2,7 \\
\hline & EGB5 & $31 \mathrm{a}$ & 6,7 & $99 \mathrm{a}$ & 9,1 & $61 \mathrm{a}$ & 1,5 & $122 \mathrm{a}$ & 5,0 \\
\hline & Bocage & $37 \mathrm{a}$ & 6,0 & $99 \mathrm{a}$ & 8,9 & $66 \mathrm{a}$ & 5,0 & $125 \mathrm{a}$ & 6,8 \\
\hline \multicolumn{10}{|c|}{$2 \mathrm{HVJ}(2007)$} \\
\hline \multirow{3}{*}{ früh } & Licarta & $29 \mathrm{a}$ & 5,7 & $83 \mathrm{a}$ & 5,4 & $67 \mathrm{a}$ & 5,1 & $122 \mathrm{a}$ & 9,5 \\
\hline & Abersilo & $36 \mathrm{a}$ & 5,4 & $88 \mathrm{a}$ & 8,7 & $68 \mathrm{a}$ & 2,7 & 129 a & 11,7 \\
\hline & EGB1 & $32 \mathrm{a}$ & 2,7 & $92 \mathrm{a}$ & 5,8 & $61 \mathrm{a}$ & 4,9 & $120 \mathrm{a}$ & 10,7 \\
\hline \multirow{6}{*}{ mittel } & Respect & $32 \mathrm{ab}$ & 3,8 & $93 \mathrm{a}$ & 12,3 & $67 \mathrm{a}$ & 2,6 & $127 \mathrm{a}$ & 10,8 \\
\hline & Betty & $31 \mathrm{ab}$ & 1,9 & $95 \mathrm{a}$ & 3,7 & $57 \mathrm{ab}$ & 3,3 & $126 \mathrm{a}$ & 11,6 \\
\hline & Aberdart & $28 \mathrm{ab}$ & 3,7 & $85 \mathrm{a}$ & 4,2 & $65 \mathrm{a}$ & 3,4 & 129 a & 5,5 \\
\hline & Rodrigo & $34 \mathrm{a}$ & 3,6 & $93 \mathrm{a}$ & 7,7 & $58 \mathrm{ab}$ & 4,7 & $118 \mathrm{a}$ & 3,7 \\
\hline & EGB2 & $23 \mathrm{~b}$ & 1,3 & $95 \mathrm{a}$ & 6,9 & $49 \mathrm{~b}$ & 1,1 & $110 \mathrm{a}$ & 3,2 \\
\hline & Aubisque & $28 \mathrm{ab}$ & 5,5 & $86 \mathrm{a}$ & 3,8 & $53 \mathrm{~b}$ & 5,5 & $104 \mathrm{a}$ & 14,7 \\
\hline \multirow{6}{*}{ spät } & Sponsor & $23 \mathrm{a}$ & 1,5 & $86 \mathrm{a}$ & 2,5 & $51 \mathrm{a}$ & 3,0 & $111 \mathrm{ab}$ & 4,6 \\
\hline & AberAvon & $23 \mathrm{a}$ & 1,0 & $78 \mathrm{a}$ & 2,9 & $46 \mathrm{a}$ & 4,3 & $105 \mathrm{ab}$ & 11,0 \\
\hline & EGB3 & $20 \mathrm{a}$ & 4,6 & $79 \mathrm{a}$ & 12,3 & $50 \mathrm{a}$ & 4,6 & $119 \mathrm{a}$ & 9,9 \\
\hline & EGB4 & $21 \mathrm{a}$ & 2,0 & $95 \mathrm{a}$ & 0,8 & $49 \mathrm{a}$ & 6,8 & $110 \mathrm{a} b$ & 10,4 \\
\hline & EGB5 & $18 \mathrm{a}$ & 4,0 & $75 \mathrm{a}$ & 3,6 & $31 \mathrm{~b}$ & 4,5 & $86 \mathrm{~b}$ & 9,4 \\
\hline & Bocage & $23 \mathrm{a}$ & 5,6 & $90 \mathrm{a}$ & 14,6 & $38 \mathrm{ab}$ & 6,6 & $92 \mathrm{~b}$ & 8,9 \\
\hline
\end{tabular}

signifikante Unterschiede gelten für gruppierte Zellen mit unterschiedlichen Buchstaben $(\mathrm{P}<0,05), \quad \mathrm{SD}=$ Standardabweichung. 
Tab. 13: TM-Ertrag (dt/ha) in Abhängigkeit von den Sortenreifegruppen (früh, mittel und spät) von Deutschen Weidelgras am Versuchsstandort HS im 1HVJ (2006) und im 2HVJ (2007).

\begin{tabular}{|c|c|c|c|c|c|c|c|c|c|}
\hline \multicolumn{10}{|c|}{ 1HVJ (2006) } \\
\hline \multirow{3}{*}{\multicolumn{2}{|c|}{$\begin{array}{l}\text { Reifegruppe/ } \\
\text { Sorten }\end{array}$}} & \multicolumn{8}{|c|}{ Schnittzeitpunkt } \\
\hline & & \multicolumn{4}{|c|}{ FSV } & \multicolumn{4}{|c|}{ SSV } \\
\hline & & $\begin{array}{l}1 . \\
\text { Schnitt }\end{array}$ & SD & Jahresertrag & SD & $\begin{array}{l}1 . \\
\text { Schnitt }\end{array}$ & SD & Jahresertrag & $\mathrm{SD}$ \\
\hline \multirow{3}{*}{ früh } & Licarta & $56 \mathrm{a}$ & 5,2 & $123 \mathrm{a}$ & 12,1 & $65 \mathrm{a}$ & 0,6 & $145 \mathrm{~b}$ & 1,5 \\
\hline & Abersilo & $60 \mathrm{a}$ & 3,1 & 139 a & 12,2 & $73 \mathrm{a}$ & 3,2 & $162 \mathrm{ab}$ & 2,5 \\
\hline & EGB1 & $63 \mathrm{a}$ & 4,2 & $148 \mathrm{a}$ & 7,2 & $73 \mathrm{a}$ & 5,8 & $163 \mathrm{a}$ & 11,5 \\
\hline \multirow{6}{*}{ mittel } & Respect & $57 \mathrm{a}$ & 2,6 & $141 \mathrm{a}$ & 11,6 & $66 \mathrm{a}$ & 3,5 & $158 \mathrm{a}$ & 4,7 \\
\hline & Betty & $56 \mathrm{a}$ & 3,2 & $127 \mathrm{a}$ & 6,6 & $71 \mathrm{a}$ & 8,0 & $154 \mathrm{a}$ & 7,6 \\
\hline & Aberdart & $57 \mathrm{a}$ & 3,1 & $141 \mathrm{a}$ & 12,0 & $68 \mathrm{a}$ & 8,7 & $157 \mathrm{a}$ & 7,2 \\
\hline & Rodrigo & $56 \mathrm{a}$ & 3,6 & $144 \mathrm{a}$ & 9,8 & $68 \mathrm{a}$ & 1,0 & $163 \mathrm{a}$ & 4,0 \\
\hline & EGB2 & $44 \mathrm{~b}$ & 3,0 & 129 a & 7,2 & $63 \mathrm{a}$ & 2,1 & $157 \mathrm{a}$ & 7,0 \\
\hline & Aubisque & $55 \mathrm{a}$ & 0,6 & $141 \mathrm{a}$ & 1,0 & $69 \mathrm{a}$ & 3,4 & $162 \mathrm{a}$ & 1,7 \\
\hline \multirow{6}{*}{ spät } & Sponsor & $46 \mathrm{ab}$ & 1,7 & $130 \mathrm{a}$ & 7,0 & $62 \mathrm{ab}$ & 2,0 & 155 a b & 3,5 \\
\hline & AberAvon & $51 \mathrm{a}$ & 3,2 & $138 \mathrm{a}$ & 6,6 & $66 \mathrm{a}$ & 1,5 & $161 \mathrm{a}$ & 3,6 \\
\hline & EGB3 & $48 \mathrm{a} \mathrm{b}$ & 2,3 & $133 \mathrm{a}$ & 4,4 & $61 \mathrm{a} \mathrm{b}$ & 1,5 & $152 \mathrm{ab}$ & 7,2 \\
\hline & EGB4 & $46 \mathrm{a} \mathrm{b}$ & 3,5 & $140 \mathrm{a}$ & 9,8 & $61 \mathrm{a} \mathrm{b}$ & 2,1 & $157 a b$ & 5,9 \\
\hline & EGB5 & $40 \mathrm{~b}$ & 4,2 & $129 \mathrm{a}$ & 11,3 & 59 a b & 1,5 & $154 \mathrm{a} \mathrm{b}$ & 8,1 \\
\hline & Bocage & $43 \mathrm{a} \mathrm{b}$ & 3,8 & $124 \mathrm{a}$ & 4,7 & $55 \mathrm{~b}$ & 1,5 & $144 \mathrm{~b}$ & 6,1 \\
\hline \multicolumn{10}{|c|}{$2 \mathrm{HVJ}(2007)$} \\
\hline \multirow{3}{*}{ früh } & Licarta & $68 \mathrm{a}$ & 7,9 & $152 \mathrm{a}$ & 7,2 & $82 \mathrm{a}$ & 3,8 & $172 \mathrm{a}$ & 6,5 \\
\hline & Abersilo & $49 \mathrm{~b}$ & 4,0 & $138 \mathrm{a}$ & 11,1 & $84 \mathrm{a}$ & 3,8 & $178 \mathrm{a}$ & 9,8 \\
\hline & EGB1 & $57 \mathrm{ab}$ & 3,8 & $147 \mathrm{a}$ & 6,6 & $80 \mathrm{a}$ & 7,6 & $180 \mathrm{a}$ & 10,1 \\
\hline \multirow{6}{*}{ mittel } & Respect & $52 \mathrm{a}$ & 6,8 & $141 \mathrm{a}$ & 8,5 & $82 \mathrm{a}$ & 9,0 & $177 \mathrm{a}$ & 15,5 \\
\hline & Betty & $62 \mathrm{a}$ & 7,4 & $150 \mathrm{a}$ & 9,5 & $82 \mathrm{a}$ & 3,5 & 179 a & 6,1 \\
\hline & Aberdart & $51 \mathrm{a}$ & 5,3 & $135 \mathrm{a}$ & 2,3 & 75 a b & 5,9 & $168 \mathrm{a}$ & 12,8 \\
\hline & Rodrigo & $50 \mathrm{a}$ & 3,8 & $142 \mathrm{a}$ & 3,5 & $79 \mathrm{a} \mathrm{b}$ & 6,7 & $179 \mathrm{a}$ & 9,1 \\
\hline & EGB2 & $52 \mathrm{a}$ & 11,2 & $138 \mathrm{a}$ & 16,3 & $63 \mathrm{~b}$ & 1,5 & $159 \mathrm{a}$ & 6,7 \\
\hline & Aubisque & $49 \mathrm{a}$ & 11,2 & $132 \mathrm{a}$ & 10,5 & 80 a & 5,7 & $175 \mathrm{a}$ & 10,7 \\
\hline \multirow{6}{*}{ spät } & Sponsor & $53 \mathrm{a}$ & 8,2 & $143 \mathrm{a}$ & 7,0 & $70 \mathrm{a}$ & 5,0 & $171 \mathrm{a}$ & 9,5 \\
\hline & AberAvon & $50 \mathrm{ab}$ & 1,5 & $137 \mathrm{a}$ & 2,5 & $68 \mathrm{a}$ & 5,0 & $166 \mathrm{a}$ & 4,0 \\
\hline & EGB3 & $49 \mathrm{a} \mathrm{b}$ & 4,4 & $141 \mathrm{a}$ & 9,6 & $67 \mathrm{a} b$ & 3,5 & $168 \mathrm{a}$ & 5,3 \\
\hline & EGB4 & $50 \mathrm{ab}$ & 3,0 & $146 \mathrm{a}$ & 3,6 & $66 \mathrm{a} b$ & 4,6 & $172 \mathrm{a}$ & 8,1 \\
\hline & EGB5 & $38 \mathrm{~b}$ & 3,5 & $128 \mathrm{a}$ & 8,7 & $56 \mathrm{~b}$ & 2,6 & 159 a & 3,5 \\
\hline & Bocage & $40 \mathrm{~b}$ & 3,6 & $125 \mathrm{a}$ & 13,0 & 59 a b & 3,8 & $161 \mathrm{a}$ & 12,9 \\
\hline
\end{tabular}

signifikante Unterschiede gelten für gruppierte Zellen mit unterschiedlichen Buchstaben $(\mathrm{P}<0,05)$, $\mathrm{SD}=$ Standardabweichung. 
Tab. 14: TM-Ertrag (dt/ha) in Abhängigkeit von den Sortenreifengruppen (früh, mittel und spät) des Deutschen Weidelgrases, im Mittel der beiden Versuchsjahren (1HVJ und 2HVJ) und der beiden Versuchsstandorten (BS und HS), im Jahresmittel.

\begin{tabular}{|c|c|c|c|c|c|c|c|}
\hline \multirow{3}{*}{\multicolumn{2}{|c|}{$\begin{array}{l}\text { Reifegruppe/ } \\
\text { Sorten }\end{array}$}} & \multicolumn{6}{|c|}{ Schnittzeitpunkt } \\
\hline & & \multicolumn{3}{|c|}{ FSV } & \multicolumn{3}{|c|}{ FSV } \\
\hline & & TM-Ertr & & SD & TM-Ertr & & SD \\
\hline \multirow{3}{*}{ früh } & Licarta & 117,7 & $\mathrm{a}$ & 4,0 & 145,7 & $\bar{a}$ & 5,7 \\
\hline & Abersilo & 121,0 & $\mathrm{a}$ & 7,5 & 154,3 & $\bar{a}$ & 6,0 \\
\hline & EGB1 & 125,0 & $\mathrm{a}$ & 3,0 & 152,0 & $\bar{a}$ & 4,4 \\
\hline \multirow{6}{*}{ mittel } & Respect & 123,0 & $\mathrm{a}$ & 8,7 & 150,3 & $\mathrm{a}$ & 6,6 \\
\hline & Betty & 121,0 & $\mathrm{a}$ & 1,0 & 150,7 & $\mathrm{a}$ & 3,2 \\
\hline & Aberdart & 118,7 & $\mathrm{a}$ & 2,9 & 149,7 & $a b$ & 1,5 \\
\hline & Rodrigo & 125,0 & $\mathrm{a}$ & 1,7 & 152,0 & $\mathrm{a}$ & 3,6 \\
\hline & EGB2 & 116,3 & $\mathrm{a}$ & 4,0 & 137,3 & $\mathrm{~b}$ & 3,2 \\
\hline & Aubisque & 118,0 & $\mathrm{a}$ & 4,4 & 142,7 & $a b$ & 6,8 \\
\hline \multirow{6}{*}{ spät } & Sponsor & 117,0 & $\mathrm{ab}$ & 2,6 & 141,7 & $\bar{a}$ & 3,5 \\
\hline & AberAvon & 115,7 & $\mathrm{ab}$ & 3,1 & 141,0 & $\mathrm{a}$ & 5,3 \\
\hline & EGB3 & 114,7 & $\mathrm{ab}$ & 6,1 & 142,0 & $\mathrm{a}$ & 7,5 \\
\hline & EGB4 & 123,0 & $\mathrm{a}$ & 4,4 & 142,3 & $\mathrm{a}$ & 2,1 \\
\hline & EGB5 & 107,3 & $\mathrm{~b}$ & 2,3 & 130,0 & $\mathrm{a}$ & 5,0 \\
\hline & Bocage & 109,0 & $\mathrm{~b}$ & 6,1 & 130,3 & $\mathrm{a}$ & 8,1 \\
\hline
\end{tabular}

Signifikante Unterschiede gelten für gruppierte Zellen mit unterschiedlichen Buchstaben $(\mathrm{P}<0,05)$, $\mathrm{SD}=$ Standardabweichung. 


\subsubsection{Wasserlösliche Kohlenhydrate}

Am Versuchsstandort BS wiesen die Wasserlöslichen Kohlenhydrate (WlK) im Mittel der Sorten im $\underline{1 .}$ Schnitt der FSV keine signifikanten Unterschiede zwischen 1HVJ (2006) und 2HVJ (2007) auf. In der SSV lagen die WlK im 1HVJ mit 25,5 \% i.d. TM deutlich über dem Ergebnis des 2HVJ (2007) mit 14,4 \% i.d. TM. Im Jahresmittel lagen die WlK im Mittel der Sorten in der FSV im 1HVJ mit 15,4 \% i.d. TM über dem Ergebnis des $2 \mathrm{HVJ}$ mit 14,7 \% i.d. TM und in der SSV im 1HVJ mit 13,8 \% i.d. TM auch über dem Ergebnis des 2HVJ mit 11,7 \% i.d. TM (Tab. 15).

Am Versuchsstandort HS lagen die WlK im Mittel der Sorten im $\underline{1 .}$ Schnitt der FSV im 1HVJ mit 16,5 \% i.d. TM deutlich über dem Ergebnis des 2HVJ mit 13,6 \% i.d. TM. Dagegen waren die WIK in der SSV niedriger, sie lagen im Mittel der Sorten im 1HVJ mit 14,2 \% i.d. TM unter dem Ergebnis des 2HVJ mit 16,5 \% i.d. TM. Im Jahresmittel lagen die WlK im Mittel der Sorten in der FSV im 1HVJ mit 17,4 \% i.d. TM deutlich über dem Ergebnis des $2 \mathrm{HVJ}$ mit 12,1 \% i.d. TM und in der SSV im 1HVJ mit 15,4 \% i.d. TM auch über dem Ergebnis des 2HVJ mit 14,8 \% i.d. TM (Tab. 15).

Im Mittel der beiden Versuchsstandorten (BS und HS) im Mittel der Sorten im Jahresmittel der FSV lagen die WlK im 1HVJ mit 16,4 \% i.d. TM über dem Ergebnis des 2HVJ mit 15,0 \% i.d. TM. Auch lagen die WIK im Mittel der Sorten im Jahresmittel der SSV im 1HVJ mit 14,6 \% i.d. TM deutlich über dem Ergebnis des 2HVJ mit 11,9 \% i.d. TM.

Tab. 15: Versuchsjahresvergleich (2006/2007) für wasserlösliche Kohlenhydrate (\% i.d. TM) Mittelwert aus allen Sorten im 1. Schnitt und im Jahresmittel.

\begin{tabular}{|c|c|c|c|c|c|c|c|c|c|c|c|c|}
\hline \multicolumn{13}{|l|}{ 1. Schnitt } \\
\hline \multirow{3}{*}{ Versuchsjahr } & \multicolumn{6}{|c|}{ BS } & \multicolumn{6}{|c|}{ HS } \\
\hline & \multicolumn{3}{|c|}{ FSV } & \multicolumn{3}{|c|}{ SSV } & \multicolumn{3}{|c|}{ FSV } & \multicolumn{3}{|c|}{ SSV } \\
\hline & Mw & & SD & Mw & & SD & Mw & & SD & $\mathrm{Mw}$ & & SD \\
\hline 2006 & 21,6 & $\mathrm{a}$ & 5,9 & 25,5 & $\mathrm{a}$ & 6,0 & 16,5 & $\mathrm{a}$ & 3,9 & 14,2 & $\mathrm{~b}$ & 3,8 \\
\hline 2007 & 21,5 & $\mathrm{a}$ & 5,2 & 14,4 & $\mathrm{~b}$ & 4,6 & 13,6 & $\mathrm{~b}$ & 3,1 & 16,5 & $\mathrm{a}$ & 4,2 \\
\hline \multicolumn{13}{|l|}{ Jahresmittel } \\
\hline 2006 & 15,4 & $\mathrm{a}$ & 3,1 & 13,8 & $\mathrm{a}$ & 2,6 & 17,4 & $\mathrm{a}$ & 2,7 & 15,4 & $\mathrm{a}$ & 2,1 \\
\hline 2007 & 14,7 & $\mathrm{~b}$ & 2,8 & 11,7 & $\mathrm{~b}$ & 2,6 & 12,1 & $\mathrm{~b}$ & 2,6 & 14,8 & $\mathrm{~b}$ & 3,1 \\
\hline
\end{tabular}

Signifikante Unterschiede gelten für Werte in derselben Spalte mit unterschiedlichen Buchstaben bei $\mathrm{P}<0,05$, SD = Standardabweichung, Mw = Mittelwert aus allen 20 Sorten und 3 Wiederholungen .

Am Versuchsstandort BS waren die WlK im Mittel der Sorten im 1. Schnitt des 1HVJ bei der SSV höher als in der FSV. Dagegen lagen die WlK im 2HVJ bei der FSV deutlich über dem Ergebnis der SSV. Im Jahresmittel lagen die WIK im Mittel der Sorten in den beiden Versuchsjahren 1HVJ und 2HVJ bei der FSV höher bei der SSV (Tab. 16). 
Am Versuchsstandort HS lagen die WIK im Mittel der Sorten im 1. Schnitt des 1HVJ bei der FSV über dem Ergebnis der SSV. Im 2HVJ waren die WIK bei der SSV deutlich höher als in der FSV. Im Jahresmittel lagen die WIK im Mittel der Sorten im 1. Schnitt des 1HVJ bei der FSV über dem Ergebnis der SSV. Im 2HVJ dagegen waren die WlK in der SSV deutlich höher als in der FSV (Tab. 16).

Im Mittel der beiden Versuchjahre (1HVJ und 2HVJ) und der beiden Versuchsstandorten (BS und HS) waren die WIK im Mittel der Sorten im Jahresmittel bei der FSV (15,7 \% i.d. TM) deutlich höher als in der SSV (13,3 \% i.d. TM).

Tab. 16: Schnittzeitpunktvergleich (FSV/SSV) für wasserlösliche Kohlenhydrate (\% i.d. TM) Mittelwert aus allen Sorten im 1. Schnitt und im Jahresmittel.

\begin{tabular}{|c|c|c|c|c|c|c|c|c|c|c|c|c|}
\hline \multicolumn{13}{|l|}{ 1. Schnitt } \\
\hline \multirow{3}{*}{ Schnittzeitpunkt } & \multicolumn{6}{|c|}{ BS } & \multicolumn{6}{|c|}{ HS } \\
\hline & \multicolumn{3}{|c|}{$1 \mathrm{HVJ}$} & \multicolumn{3}{|c|}{ 2HVJ } & \multicolumn{3}{|c|}{$1 \mathrm{HVJ}$} & \multicolumn{3}{|c|}{$2 \mathrm{HVJ}$} \\
\hline & Mw & & SD & Mw & & SD & Mw & & SD & $\mathrm{Mw}$ & & SD \\
\hline FSV & 21,6 & $\mathrm{~b}$ & 5,9 & 21,6 & $\mathrm{a}$ & 5,2 & 16,5 & $\mathrm{a}$ & 3,9 & 13,6 & $\mathrm{~b}$ & 3,1 \\
\hline SSV & 25,5 & $\mathrm{a}$ & 6,1 & 14,4 & $\mathrm{~b}$ & 4,5 & 14,2 & $\mathrm{~b}$ & 3,8 & 16,5 & $\mathrm{a}$ & 4,2 \\
\hline \multicolumn{13}{|l|}{ Jahresmittel } \\
\hline FSV & 15,4 & $\mathrm{a}$ & 3,1 & 15,1 & $\mathrm{a}$ & 3,1 & 17,4 & $\mathrm{a}$ & 2,7 & 12,1 & $\mathrm{~b}$ & 2,6 \\
\hline SSV & 13,8 & $\mathrm{~b}$ & 2,6 & 11,7 & $\mathrm{~b}$ & 2,6 & 15,4 & $\mathrm{~b}$ & 2,1 & 14,8 & $\mathrm{a}$ & 3,1 \\
\hline
\end{tabular}

Signifikante Unterschiede gelten für Werte in derselben Spalte mit unterschiedlichen Buchstaben bei $\mathrm{P}<0,05$, SD = Standardabweichung, $\mathrm{Mw}=$ Mittelwert aus allen 20 Sorten und 3 Wiederholungen .

Die WlK waren in der FSV und SSV des 1HVJ und in der FSV des 2HVJ am Versuchsstandort BS höher als am Versuchsstandort HS. In der SSV des 2HVJ waren die WIK am Versuchsstandort HS höher als am Versuchsstandort BS (Tab. 17).

Im Mittel der beiden Versuchsjahren (1HVJ und 2HVJ) waren die WlK im 1. Schnitt der FSV am Versuchsstandort BS (21,6 i.d. TM) deutlich höher als am Versuchsstandort HS(16,5 i.d. TM). Auch waren die WIK in der SSV am Versuchsstandort BS (20,0 i.d. TM) höher als am Versuchsstandort HS (13,9 i.d. TM).

Tab. 17: Wasserlösliche Kohlenhydrate (\% i.d. TM) an beiden Versuchsstandorte (BS und HS) im 1. Schnitt.

\begin{tabular}{|c|c|c|c|c|c|c|c|c|c|c|c|c|}
\hline \multirow{3}{*}{ Standort } & \multicolumn{6}{|c|}{$1 \mathrm{HVJ}$} & \multicolumn{6}{|c|}{$2 \mathrm{HVJ}$} \\
\hline & \multicolumn{3}{|c|}{ FSV } & \multicolumn{3}{|c|}{ SSV } & \multicolumn{3}{|c|}{ FSV } & \multicolumn{3}{|c|}{ SSV } \\
\hline & Mw & & SD & Mw & & SD & $\mathrm{Mw}$ & & SD & Mw & & SD \\
\hline BS & 21,6 & $\mathrm{a}$ & 5,9 & 25,5 & $\mathrm{a}$ & 6,0 & 21,6 & $\mathrm{a}$ & 5,2 & 14,4 & b & 4,6 \\
\hline HS & 16,5 & $\mathrm{~b}$ & 3,9 & 14,2 & $\mathrm{~b}$ & 3,8 & 13,6 & $\mathrm{~b}$ & 3,1 & 16,5 & $\mathrm{a}$ & 4,2 \\
\hline
\end{tabular}

Signifikante Unterschiede gelten für Werte in derselben Spalte mit unterschiedlichen Buchstaben bei $\mathrm{P}<0,05$, $\mathrm{SD}=$ Standardabweichung, Mw = Mittelwert aus allen 20 Sorten und 3 Wiederholungen. 
Tabellen 18 und 19 zeigen die Unterschiede zwischen den Sorten in den Reifegruppen (früh, mittel und spät).

Reifegruppe „früh“:

Am Versuchsstandort BS wiesen die WIK im 1. Schnitt und im Jahresmittel der FSV und der SSV des 1HVJ sowie im 1. Schnitt der FSV und der SSV des 2HVJ keine signifikanten Unterschiede zwischen den Sorten (Licarta, Abersilo und EGB1) in der Reifegruppe „früh“ auf. Im Jahresmittel der SSV des 2HVJ waren die WlK der Sorte Licarta niedriger als die WlK der Sorte Abersilo (Tab. 18).

Am Versuchsstandort HS wiesen die WIK im 1. Schnitt und im Jahresmittel der FSV und der SSV des 1HVJ und im 1. Schnitt und im Jahresmittel der FSV des 2HVJ keine signifikanten Unterschiede zwischen den Sorten in der Reifegruppe „früh“ auf. Im 1. Schnitt und im Jahresmittel in der SSV des 2HVJ waren die WlK der Sorte Licarta niedriger als die WlK der Sorte Abersilo (Tab. 19).

Im Mittel der beiden Versuchsjahren (1HVJ und 2HVJ) und der beiden Versuchsstandorten (BS und HS) wiesen die WlK im Jahresmittel der FSV keine signifikanten Unterschiede zwischen den Sorten (Licarta, Abersilo und EGB1) in der Reifegruppe „früh“ auf. Im Jahresmittel der SSV lagen die WlK der Sorte Licarta (13,2 \% i.d. TM) niedriger als die WlK der Sorten EGB1 (14,4 \% i.d. TM) und die Sorte Abersilo (15,3 \% i.d. TM) (Tab. 20).

\section{$\underline{\text { Reifegruppe „mittel“: }}$}

Am Versuchsstandort BS wiesen die WIK im 1. Schnitt der FSV und der SSV des 1HVJ und im Jahresmittel der SSV des 2HVJ keine signifikanten Unterschiede zwischen den Sorten (Respect, Betty, Aberdart, Rodrigo, EGB2 und Aubisque) in der Reifegruppe „mittel“ auf. Im Jahresmittel der FSV und SSV des 1HVJ gab es signifikante Unterschiede zwischen den Sorten; die Sorte Aberdart hatte den höchsten WlK (18,5 \% i.d. TM), die Sorte Respect wies den niedrigsten WIK (15,4 \% i.d. TM) auf. Im 1. Schnitt der FSV und der SSV des 2HVJ und im Jahresmittel der FSV des 2HVJ gab es signifikante Unterschiede zwischen den Sorten; die WlK waren in der Sorte Aubisque am höchsten, in der Sorte Respect am niedrigsten (Tab.18). Am Versuchsstandort HS wiesen die WIK im 1. Schnitt und im Jahresmittel der FSV und der SSV des 1HVJ sowie im 1. Schnitt und im Jahresmittel der FSV des 2HVJ keine signifikanten Unterschiede zwischen den Sorten in der Reifegruppe „mittel“ auf. Im 1. Schnitt der SSV des 2HVJ gab es signifikante Unterschiede zwischen den Sorten; die Sorte Aberdart hatte den höchsten WlK (17,8 \% i.d. TM), die Sorte Betty den niedrigsten (12,7 \% i.d. TM). 
Auch im Jahresmittel der SSV des 2HVJ gab es signifikante Unterschiede zwischen den Sorten. Die Sorte Aberdart zeigte den höchsten WlK (15,2 \% i.d. TM), die Sorte Respect den niedrigsten WlK (11,7 \% i.d. TM) (Tab. 19).

Im Mittel der beiden Versuchsjahren (1HVJ und 2HVJ) und der beiden Versuchsstandorten (BS und HS) wiesen die WlK im Jahresmittel der FSV signifikante Unterschiede zwischen den Sorten (Respect, Betty, Aberdart, Rodrigo, EGB2 und Aubisque) in der Reifegruppe „mittel“ auf; die Sorte Aberdart hatte den höchsten WlK (18,5 \% i.d. TM), die Sorte Respect wies den niedrigsten WIK (15,5 \% i.d. TM) auf. Auch im Jahresmittel der SSV wiesen die WlK signifikante Unterschiede zwischen den Sorten; die Sorte Aberdart hatte den höchsten WlK (15,5 \% i.d. TM), die Sorte Respect wies den niedrigsten WlK (12,8 \% i.d. TM) auf (Tab. 20).

\section{Reifegruppe ,spät“:}

Am Versuchsstandort BS wiesen die WlK im 1. Schnitt und im Jahresmittel der FSV des 1HVJ sowie im 1. Schnitt der FSV des 2HVJ keine signifikanten Unterschiede zwischen den Sorten (Sponsor, AberAvon, EGB3, EGB4, EGB5 und Bocage) in der Reifegruppe „spät“ auf.

Im 1. Schnitt der SSV des $1 \mathrm{HVJ}$ gab es signifikante Unterschiede zwischen den Sorten; die Sorte EGB5 hatte den höchsten WIK (33 \% i.d. TM), die Sorte EGB3 die niedrigsten (25,3 \% i.d. TM). Im Jahresmittel der SSV des 1HVJ gab es signifikante Unterschiede zwischen den Sorten. Hier zeigte die Sorte AberAvon die höchsten WlK (15,2 \% i.d. TM), die Sorten EGB3 und EGB4 die niedrigsten (13,7 \% i.d. TM). Im Jahresmittel der FSV des 2HVJ sowie im 1. Schnitt und im Jahresmittel der SSV des 2HVJ gab es signifikante Unterschiede zwischen den Sorten; die Sorte EGB5 hatte die höchsten, die Sorten Sponsor und EGB3 hatten die niedrigsten WlK (Tab. 18).

Am Versuchsstandort HS wiesen die WlK im 1. Schnitt der FSV des 1HVJ signifikante Unterschiede zwischen den Sorten auf, die Sorte EGB5 hatte die höchste WlK (21,9 \% i.d. TM), die Sorte EGB4 die niedrigste (12,7 \% i.d. TM). Auch im Jahresmittel der FSV des 1HVJ wiesen die WlK signifikante Unterschiede zwischen den Sorten auf, die Sorte AberAvon hatte die höchste WIK (20,8 \% i.d. TM), die Sorte Sponsor die niedrigste (16,1 \% i.d. TM). Im 1. Schnitt der SSV des 1HVJ wiesen die WlK signifikante Unterschiede zwischen den Sorten auf, die Sorte Bocage hatte die höchste WlK (18 \% i.d. TM), die Sorte EGB3 die niedrigste (12,1 \% i.d. TM). Im Jahresmittel der SSV des $1 \mathrm{HVJ}$ wiesen die WlK ebenfalls 
signifikante Unterschiede zwischen den Sorten auf. Hier waren die WlK der Sorte EGB5 am höchsten (17,9 \% i.d. TM), der Sorten EGB3 und der Sponsor am niedrigsten (14,7 \% i.d. TM). Im 1. Schnitt der FSV und der SSV des 2HVJ wiesen die WlK keine signifikanten Unterschiede zwischen den Sorten in der Reifegruppe „spät“ auf. Im Jahresmittel der FSV des 2HVJ wiesen die WlK signifikante Unterschiede zwischen den Sorten auf. Die Sorte AberAvon zeigte die höchste WlK (18,2 \% i.d. TM), die Sorte Sponsor die niedrigste (14,3 \% i.d. TM). Auch im Jahresmittel der SSV des 2HVJ wiesen die WlK signifikante Unterschiede zwischen den Sorten auf. Die Sorte Bocage hatte die höchste WlK (14,8 \% i.d. TM), die Sorte Sponsor die niedrigste (11,4 \% i.d. TM) (Tab. 19).

Im Mittel der beiden Versuchsjahren (1HVJ und 2HVJ) und der beiden Versuchsstandorten (BS und HS) wiesen die WlK im Jahresmittel der FSV signifikante Unterschiede zwischen den Sorten (Sponsor, AberAvon, EGB3, EGB4, EGB5 und Bocage) in der Reifegruppe „spät“ auf; die Sorte AberAvon hatte den höchsten WlK (18,6 \% i.d. TM), die Sorte Sponsor wies den niedrigsten WlK (15,1 \% i.d. TM) auf. Auch im Jahresmittel der SSV wiesen die WIK signifikante Unterschiede zwischen den Sorten; die Sorte Bocage hatte den höchsten WlK (15,8 \% i.d. TM), die Sorte EGB3 wies den niedrigsten WlK (12,9 \% i.d. TM) auf (Tab. 20). 
Tab. 18: Wasserlösliche Kohlenhydrate (\% i.d. TM) in Abhängigkeit von den Sortenreifengruppen (früh, mittel und spät) des Deutschen Weidelgrases am Versuchsstandort BS im 1HVJ (2006) und im 2HVJ (2007).

\begin{tabular}{|c|c|c|c|c|c|c|c|c|c|}
\hline \multicolumn{10}{|c|}{$1 H V J(2006)$} \\
\hline \multirow{3}{*}{\multicolumn{2}{|c|}{$\begin{array}{l}\text { Reifegruppe/ } \\
\text { Sorten }\end{array}$}} & \multicolumn{8}{|c|}{ Schnittzeitpunkt } \\
\hline & & \multicolumn{4}{|c|}{ FSV } & \multicolumn{4}{|c|}{ SSV } \\
\hline & & $\begin{array}{l}1 . \\
\text { Schnitt }\end{array}$ & SD & Jahresmittel & SD & $\begin{array}{l}1 . \\
\text { Schnitt }\end{array}$ & SD & Jahresmittel & SD \\
\hline \multirow{3}{*}{ früh } & Licarta & $21,5 \mathrm{a}$ & 1,9 & $15,5 \mathrm{a}$ & 1,0 & $24,0 \mathrm{a}$ & 2,7 & $13,5 \mathrm{a}$ & 0,9 \\
\hline & Abersilo & $21,7 \mathrm{a}$ & 0,7 & $17,1 \mathrm{a}$ & 0,9 & $26,8 \mathrm{a}$ & 2,1 & $16,0 \mathrm{a}$ & 0,9 \\
\hline & EGB1 & $24,2 \mathrm{a}$ & 0,1 & $17,6 \mathrm{a}$ & 0,2 & $27,2 \mathrm{a}$ & 1,7 & $14,9 \mathrm{a}$ & 0,9 \\
\hline \multirow{6}{*}{ mittel } & Respect & $24,0 \mathrm{a}$ & 0,9 & $15,4 \mathrm{~b}$ & 0,4 & $26,3 \mathrm{a}$ & 0,7 & $13,0 \mathrm{~b}$ & 0,1 \\
\hline & Betty & $23,6 \mathrm{a}$ & 1,1 & $17,1 \mathrm{a} b$ & 0,8 & $26,4 \mathrm{a}$ & 1,3 & 14,2 a b & 0,2 \\
\hline & Aberdart & $25,7 \mathrm{a}$ & 1,8 & $18,5 \mathrm{a}$ & 0,4 & $27,8 \mathrm{a}$ & 1,0 & $15,7 \mathrm{a}$ & 0,6 \\
\hline & Rodrigo & $25,3 \mathrm{a}$ & 0,9 & $16,3 \mathrm{~b}$ & 1,1 & $29,7 \mathrm{a}$ & 0,7 & $14,8 \mathrm{a}$ & 0,9 \\
\hline & EGB2 & $26,8 \mathrm{a}$ & 1,8 & $17,0 \mathrm{a} b$ & 0,1 & $28,9 \mathrm{a}$ & 1,0 & 14,8 a b & 0,3 \\
\hline & Aubisque & 26,0 a & 0,7 & $18,7 \mathrm{a}$ & 0,4 & $28,3 \mathrm{a}$ & 2,4 & $15,3 \mathrm{a}$ & 1,1 \\
\hline \multirow{6}{*}{ spät } & Sponsor & $24,6 \mathrm{a}$ & 0,9 & $15,6 \mathrm{a}$ & 1,1 & $30,0 \mathrm{~b}$ & 0,6 & $14,7 \mathrm{~b}$ & 0,6 \\
\hline & AberAvon & 26,0 a & 0,9 & $18,3 \mathrm{a}$ & 0,1 & $30,1 \mathrm{~b}$ & 0,9 & $17,0 \mathrm{a}$ & 0,7 \\
\hline & EGB3 & $23,8 \mathrm{a}$ & 2,6 & $15,5 \mathrm{a}$ & 0,7 & $25,3 \mathrm{c}$ & 0,2 & $13,7 \mathrm{~b}$ & 0,7 \\
\hline & EGB4 & $23,6 \mathrm{a}$ & 1,8 & $16,1 \mathrm{a}$ & 0,3 & $28,5 \mathrm{~b}$ & 0,6 & $13,7 \mathrm{~b}$ & 0,4 \\
\hline & EGB5 & $26,3 \mathrm{a}$ & 0,7 & $17,7 \mathrm{a}$ & 1,8 & $33,0 \mathrm{a}$ & 1,2 & $16,5 \mathrm{a}$ & 1,0 \\
\hline & Bocage & $25,7 \mathrm{a}$ & 2,6 & $17,2 \mathrm{a}$ & 1,2 & $32,8 \mathrm{a}$ & 0,1 & $16,5 \mathrm{a}$ & 0,2 \\
\hline \multicolumn{10}{|c|}{$2 \mathrm{HVJ}(2007)$} \\
\hline \multirow{3}{*}{ früh } & Licarta & $23,5 \mathrm{a}$ & 2,2 & $15,7 \mathrm{~b}$ & 0,8 & $13,2 \mathrm{a}$ & 1,3 & $11,5 \mathrm{~b}$ & 0,4 \\
\hline & Abersilo & $24,2 \mathrm{a}$ & 0,9 & $17,7 \mathrm{a}$ & 0,9 & $15,3 \mathrm{a}$ & 0,9 & $14,0 \mathrm{a}$ & 0,9 \\
\hline & EGB1 & $25,4 \mathrm{a}$ & 1,2 & $17,8 \mathrm{a}$ & 0,2 & $14,3 \mathrm{a}$ & 0,4 & 13,0 a b & 0,3 \\
\hline \multirow{6}{*}{ mittel } & Respect & $22,8 \mathrm{~b}$ & 1,3 & $14,8 \mathrm{~b}$ & 0,6 & $13,3 \mathrm{~b}$ & 0,3 & $11,3 \mathrm{a}$ & 0,6 \\
\hline & Betty & $23,5 \mathrm{~b}$ & 1,3 & $16,6 \mathrm{a} \mathrm{b}$ & 1,2 & $13,9 \mathrm{~b}$ & 1,1 & $11,3 \mathrm{a}$ & 0,9 \\
\hline & Aberdart & $25,0 \mathrm{ab}$ & 0,9 & $18,1 \mathrm{a}$ & 0,6 & $17,5 \mathrm{a}$ & 0,5 & $13,2 \mathrm{a}$ & 1,6 \\
\hline & Rodrigo & $23,0 \mathrm{~b}$ & 1,0 & $16,0 \mathrm{~b}$ & 0,6 & $15,1 \mathrm{a} \mathrm{b}$ & 0,7 & $13,1 \mathrm{a}$ & 0,4 \\
\hline & EGB2 & $22,9 \mathrm{~b}$ & 0,8 & $15,3 \mathrm{~b}$ & 1,0 & $15,0 \mathrm{a} \mathrm{b}$ & 1,2 & $11,7 \mathrm{a}$ & 0,5 \\
\hline & Aubisque & $27,4 \mathrm{a}$ & 0,8 & $18,3 \mathrm{a}$ & 0,1 & $18,3 \mathrm{a}$ & 2,5 & $13,6 \mathrm{a}$ & 0,6 \\
\hline \multirow{6}{*}{ spät } & Sponsor & $22,0 \mathrm{a}$ & 0,4 & $14,4 \mathrm{c}$ & 0,7 & $15,8 \mathrm{~b} \mathrm{c}$ & 0,8 & $12,0 \mathrm{c} \mathrm{d}$ & 0,4 \\
\hline & AberAvon & $24,2 \mathrm{a}$ & 0,8 & $17,1 \mathrm{a} \mathrm{b}$ & 0,5 & $14,8 \mathrm{c}$ & 1,4 & 13,6 a b c & 1,3 \\
\hline & EGB3 & $20,6 \mathrm{a}$ & 0,4 & $14,4 \mathrm{c}$ & 0,4 & $14,1 \mathrm{c}$ & 1,5 & $11,4 \mathrm{~d}$ & 1,0 \\
\hline & EGB4 & 21,5 a & 4,8 & 15,2 b c & 1,4 & $17,8 \mathrm{~b}$ & 0,3 & 12,7 b c d & 0,4 \\
\hline & EGB5 & 25,9 a & 3,0 & $17,7 \mathrm{a}$ & 0,5 & $23,1 \mathrm{a}$ & 0,9 & $14,9 \mathrm{a}$ & 0,5 \\
\hline & Bocage & 26,4 a & 4,0 & 16,9 a b c & 1,5 & $21,5 \mathrm{a}$ & 1,1 & 14,4 a b & 0,5 \\
\hline
\end{tabular}

Signifikante Unterschiede gelten für gruppierte Zellen mit unterschiedlichen Buchstaben $(\mathrm{P}<0,05), \mathrm{SD}=$ Standardabweichung. 
Tab. 19: Wasserlösliche Kohlenhydrate (\% i.d. TM) in Abhängigkeit von den Sortenreifengruppen (früh, mittel und spät) des Deutschen Weidelgrases am Versuchsstandort HS im 1HVJ (2006) und im 2HVJ (2007).

\begin{tabular}{|c|c|c|c|c|c|c|c|c|c|}
\hline \multicolumn{10}{|c|}{$1 \mathrm{HVJ}(2006)$} \\
\hline \multirow{3}{*}{\multicolumn{2}{|c|}{$\begin{array}{l}\text { Reifegruppe/ } \\
\text { Sorten }\end{array}$}} & \multicolumn{8}{|c|}{ Schnittzeitpunkt } \\
\hline & & \multicolumn{4}{|c|}{ FSV } & \multicolumn{4}{|c|}{ SSV } \\
\hline & & $\begin{array}{l}1 . \\
\text { Schnitt }\end{array}$ & SD & Jahresmittel & SD & $\begin{array}{l}1 . \\
\text { Schnitt }\end{array}$ & SD & Jahresmittel & SD \\
\hline \multirow{3}{*}{ früh } & Licarta & $19,7 \mathrm{a}$ & 1,2 & $18,1 \mathrm{a}$ & 1,0 & $16,7 \mathrm{a}$ & 2,8 & 15,9 a & 0,8 \\
\hline & Abersilo & $16,4 \mathrm{a}$ & 4,2 & $18,3 \mathrm{a}$ & 3,7 & $14,3 \mathrm{a}$ & 1,9 & $16,6 \mathrm{a}$ & 1,0 \\
\hline & EGB1 & $17,7 \mathrm{a}$ & 3,9 & $19,5 \mathrm{a}$ & 2,3 & $15,7 \mathrm{a}$ & 0,8 & $16,2 \mathrm{a}$ & 1,0 \\
\hline \multirow{6}{*}{ mittel } & Respect & $17,3 \mathrm{a}$ & 4,2 & $17,0 \mathrm{a}$ & 3,4 & $14,8 \mathrm{a}$ & 1,5 & $15,5 \mathrm{a}$ & 0,3 \\
\hline & Betty & $17,7 \mathrm{a}$ & 3,4 & $16,8 \mathrm{a}$ & 1,6 & $17,3 \mathrm{a}$ & 1,4 & 16,0 a & 0,4 \\
\hline & Aberdart & $17,3 \mathrm{a}$ & 4,3 & $19,6 \mathrm{a}$ & 2,2 & $17,3 \mathrm{a}$ & 2,8 & $17,6 \mathrm{a}$ & 0,4 \\
\hline & Rodrigo & $15,2 \mathrm{a}$ & 2,4 & $17,5 \mathrm{a}$ & 1,7 & $12,7 \mathrm{a}$ & 1,6 & $15,7 \mathrm{a}$ & 0,2 \\
\hline & EGB2 & $18,9 \mathrm{a}$ & 1,5 & $18,1 \mathrm{a}$ & 2,0 & $16,1 \mathrm{a}$ & 2,4 & $15,2 \mathrm{a}$ & 0,9 \\
\hline & Aubisque & $17,2 \mathrm{a}$ & 1,0 & $18,7 \mathrm{a}$ & 1,9 & $17,3 \mathrm{a}$ & 4,9 & $17,3 \mathrm{a}$ & 2,0 \\
\hline \multirow{6}{*}{ spät } & Sponsor & $15,3 \mathrm{a} b$ & 3,0 & $16,1 \mathrm{~b}$ & 2,5 & $13,9 \mathrm{a} \mathrm{b}$ & 2,7 & $14,7 \mathrm{a}$ & 1,2 \\
\hline & AberAvon & $18,8 \mathrm{a} \mathrm{b}$ & 3,4 & $20,8 \mathrm{a}$ & 1,6 & $17,0 \mathrm{a} \mathrm{b}$ & 0,2 & $17,0 \mathrm{a}$ & 0,4 \\
\hline & EGB3 & $17,2 \mathrm{ab}$ & 2,0 & $17,5 \mathrm{a} \mathrm{b}$ & 1,3 & $12,1 \mathrm{~b}$ & 0,4 & 14,7 a & 1,4 \\
\hline & EGB4 & $12,7 \mathrm{~b}$ & 3,9 & $16,6 \mathrm{a} b$ & 0,4 & $13,9 \mathrm{a} \mathrm{b}$ & 2,1 & $15,0 \mathrm{a}$ & 0,8 \\
\hline & EGB5 & 21,9 a & 1,7 & 19,9 a b & 1,5 & $16,6 \mathrm{a} \mathrm{b}$ & 0,7 & 17,9 a & 0,8 \\
\hline & Bocage & $18,3 \mathrm{a} b$ & 1,9 & $18,1 \mathrm{a} \mathrm{b}$ & 1,9 & $18,0 \mathrm{a}$ & 3,5 & $17,3 \mathrm{a}$ & 1,9 \\
\hline \multicolumn{10}{|c|}{$2 \mathrm{HVJ}(2007)$} \\
\hline \multirow{3}{*}{ früh } & Licarta & $16,1 \mathrm{a}$ & 2,6 & $14,6 \mathrm{a}$ & 1,2 & $11,3 \mathrm{~b}$ & 1,0 & $11,7 \mathrm{~b}$ & 1,6 \\
\hline & Abersilo & $17,2 \mathrm{a}$ & 4,0 & $16,1 \mathrm{a}$ & 2,0 & $15,8 \mathrm{a}$ & 0,5 & $14,7 \mathrm{a}$ & 0,9 \\
\hline & EGB1 & $18,3 \mathrm{a}$ & 3,6 & $17,3 \mathrm{a}$ & 1,5 & $14,5 \mathrm{a} \mathrm{b}$ & 2,5 & $13,6 \mathrm{a}$ & 1,8 \\
\hline \multirow{6}{*}{ mittel } & Respect & $15,9 \mathrm{a}$ & 2,9 & $14,4 \mathrm{a}$ & 1,2 & 13,9 a b & 0,5 & $11,7 \mathrm{c}$ & 1,9 \\
\hline & Betty & $16,8 \mathrm{a}$ & 1,9 & $15,0 \mathrm{a}$ & 1,2 & $12,7 \mathrm{~b}$ & 1,4 & $11,8 \mathrm{c}$ & 1,3 \\
\hline & Aberdart & $19,8 \mathrm{a}$ & 3,8 & $17,7 \mathrm{a}$ & 1,4 & $17,8 \mathrm{a}$ & 1,5 & $15,2 \mathrm{a}$ & 2,4 \\
\hline & Rodrigo & $18,2 \mathrm{a}$ & 2,7 & $16,6 \mathrm{a}$ & 1,1 & $15,1 \mathrm{a} \mathrm{b}$ & 1,5 & 13,5 a b c & 1,9 \\
\hline & EGB2 & $18,6 \mathrm{a}$ & 1,4 & $15,5 \mathrm{a}$ & 2,6 & $15,9 \mathrm{a} \mathrm{b}$ & 2,2 & $12,7 \mathrm{~b} \mathrm{c}$ & 2,1 \\
\hline & Aubisque & $19,3 \mathrm{a}$ & 1,7 & $17,3 \mathrm{a}$ & 1,5 & $13,6 \mathrm{~b}$ & 1,1 & 13,9 a b & 1,6 \\
\hline \multirow{6}{*}{ spät } & Sponsor & $16,7 \mathrm{a}$ & 4,7 & $14,3 \mathrm{~b}$ & 1,7 & $14,5 \mathrm{a}$ & 1,2 & $11,4 \mathrm{c}$ & 2,2 \\
\hline & AberAvon & $20,6 \mathrm{a}$ & 3,7 & $18,2 \mathrm{a}$ & 1,9 & $13,8 \mathrm{a}$ & 0,8 & $12,9 \mathrm{~b} \mathrm{c}$ & 1,3 \\
\hline & EGB3 & $17,1 \mathrm{a}$ & 1,6 & 15,0 a b & 1,5 & $12,9 \mathrm{a}$ & 3,6 & $11,7 \mathrm{c}$ & 1,5 \\
\hline & EGB4 & $18,2 \mathrm{a}$ & 3,5 & $15,1 \mathrm{a} \mathrm{b}$ & 2,2 & $15,4 \mathrm{a}$ & 2,2 & $12,3 \mathrm{~b} \mathrm{c}$ & 2,3 \\
\hline & EGB5 & $20,1 \mathrm{a}$ & 3,2 & $17,7 \mathrm{a}$ & 1,7 & $16,1 \mathrm{a}$ & 1,3 & $13,8 \mathrm{a} \mathrm{b}$ & 2,6 \\
\hline & Bocage & $18,1 \mathrm{a}$ & 0,8 & $16,1 \mathrm{a} b$ & 1,9 & $17,4 \mathrm{a}$ & 1,6 & 14,8 a & 2,4 \\
\hline
\end{tabular}

Signifikante Unterschiede gelten für gruppierte Zellen mit unterschiedlichen Buchstaben $(\mathrm{P}<0,05)$, SD = Standardabweichung. 
Tab. 20: Wasserlösliche Kohlenhydrate (WlK) (\% i.d. TM) in Abhängigkeit von den Sortenreifengruppen (früh, mittel und spät) des Deutschen Weidelgrases, im Mittel der beiden Versuchsjahren (1HVJ und 2HVJ) und der beiden Versuchsstandorten (BS und HS), im Jahresmittel.

\begin{tabular}{|c|c|c|c|c|c|c|c|}
\hline \multirow{3}{*}{\multicolumn{2}{|c|}{$\begin{array}{l}\text { Reifegruppe/ } \\
\text { Sorten }\end{array}$}} & \multicolumn{6}{|c|}{ Schnittzeitpunkt } \\
\hline & & \multicolumn{3}{|c|}{ FSV } & \multicolumn{3}{|c|}{ FSV } \\
\hline & & WlK & & SD & WlK & & SD \\
\hline \multirow{3}{*}{ früh } & Licarta & 16,0 & $\mathrm{a}$ & 1,1 & 13,2 & $\mathrm{~b}$ & 0,1 \\
\hline & Abersilo & 17,3 & $\mathrm{a}$ & 1,5 & 15,3 & $\mathrm{a}$ & 0,6 \\
\hline & EGB1 & 18,0 & $\mathrm{a}$ & 1,1 & 14,4 & $\mathrm{a}$ & 0,6 \\
\hline \multirow{6}{*}{ mittel } & Respect & 15,4 & $\mathrm{~b}$ & 1,6 & 12,8 & $\mathrm{~d}$ & 0,1 \\
\hline & Betty & 16,4 & $a b$ & 0,8 & 13,3 & $\mathrm{~cd}$ & 0,2 \\
\hline & Aberdart & 18,5 & $\mathrm{a}$ & 0,9 & 15,5 & $\mathrm{a}$ & 0,6 \\
\hline & Rodrigo & 16,6 & $\mathrm{ab}$ & 0,8 & 14,2 & bc & 0,3 \\
\hline & EGB2 & 16,5 & $\mathrm{ab}$ & 0,8 & 13,6 & $\mathrm{~cd}$ & 0,2 \\
\hline & Aubisque & 18,3 & $\mathrm{a}$ & 0,9 & 15,1 & $\mathrm{ab}$ & 0,7 \\
\hline \multirow{6}{*}{ spät } & Sponsor & 15,1 & $\mathrm{~b}$ & 1,1 & 13,2 & $\mathrm{~b}$ & 0,4 \\
\hline & AberAvon & 18,6 & $\mathrm{a}$ & 1,0 & 15,1 & $\mathrm{a}$ & 0,5 \\
\hline & EGB3 & 15,6 & $\mathrm{~b}$ & 0,2 & 12,9 & $\mathrm{~b}$ & 0,9 \\
\hline & EGB4 & 15,7 & $\mathrm{~b}$ & 0,2 & 13,4 & $\mathrm{~b}$ & 0,4 \\
\hline & EGB5 & 18,3 & $\mathrm{a}$ & 1,1 & 15,8 & $\mathrm{a}$ & 0,3 \\
\hline & Bocage & 17,1 & $\mathrm{ab}$ & 0,8 & 15,8 & $\mathrm{a}$ & 0,7 \\
\hline
\end{tabular}

Signifikante Unterschiede gelten für gruppierte Zellen mit unterschiedlichen Buchstaben $(\mathrm{P}<0,05)$, SD = Standardabweichung. 


\section{Grasarten- und Sortenunterschiede}

Deutsches Weidelgras wies die höchsten WlK auf, Knaulgras und Wiesenlieschgras enthielten dagegen die niedrigsten WlK (Abb. 16).

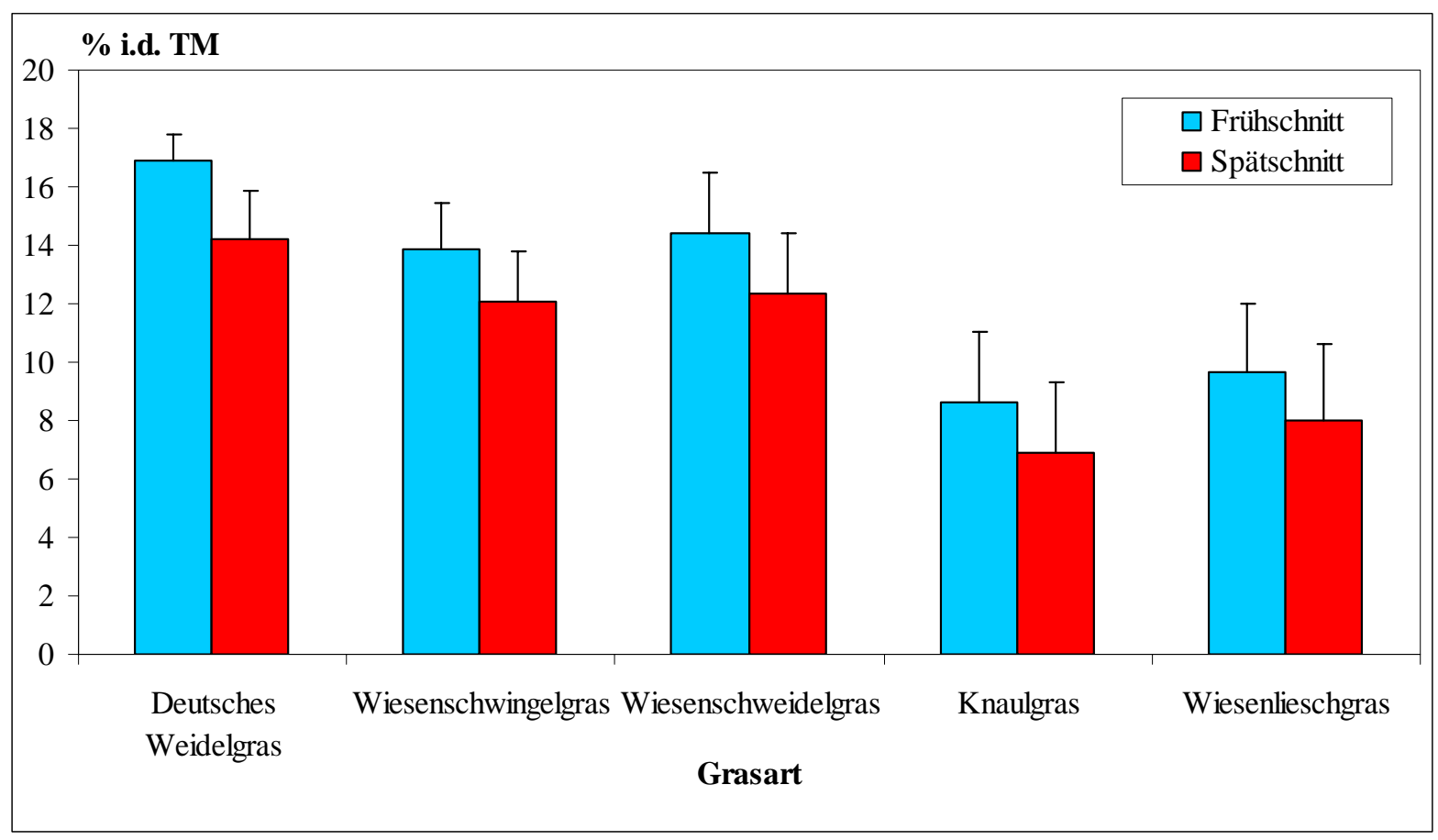

Abb. 16: Wasserlösliche Kohlenhydrate (\% i.d. TM) in verschiedenen Gräserarten des Grünlandes, Mittelwert aus den beiden Versuchsstandorten BS und HS und den beiden Versuchsjahren 1HVJ und 2HVJ bei frühem und spätem Schnittzeitpunkt.

Auch bei den Sorten innerhalb einer Grasart traten Unterschiede in den WlK auf.

Die späte Sorten (wie z.B. AberAvon) enthielt höheren WlK-Gehalt als frühe Sorten (wie z.B. Abersilo) (Abb. 17 und 18).

Die tetraploiden Sorten des Deutschen Weidelgrases (EGB5, Bocage und Aubisque) enthielten quantitativ mehr WlK als diploiden Sorten in derselben Reifegruppe (Abb. 17).

Im Mittel der beiden Versuchsjahre (1HVJ und 2HVJ) und der beiden Versuchsstandorten (BS und HS) wiesen die Sorten AberAvon (22,4 \% i.d. TM) und Aberdart (22 \% i.d. TM) in der Diploidegruppe im 1. Schnitt der FSV die höchsten WlK-Gehalte auf. Im 1. Schnitt der SSV wiesen die Sorten Aberdart (20,1 \% i.d. TM), EGB2 (19 \% i.d. TM), AberAvon (18,9 \% i.d. TM) und EGB4 (18,9 \% i.d. TM) die höchsten WlK-Gehalte auf (Abb. 17).

Im Jahresmittel der FSV und der SSV wiesen die Sorten AberAvon, Aberdart, Abersilo und EGB1 in der Diploidegruppe die höchsten WlK-Gehalte auf (Abb. 18). 


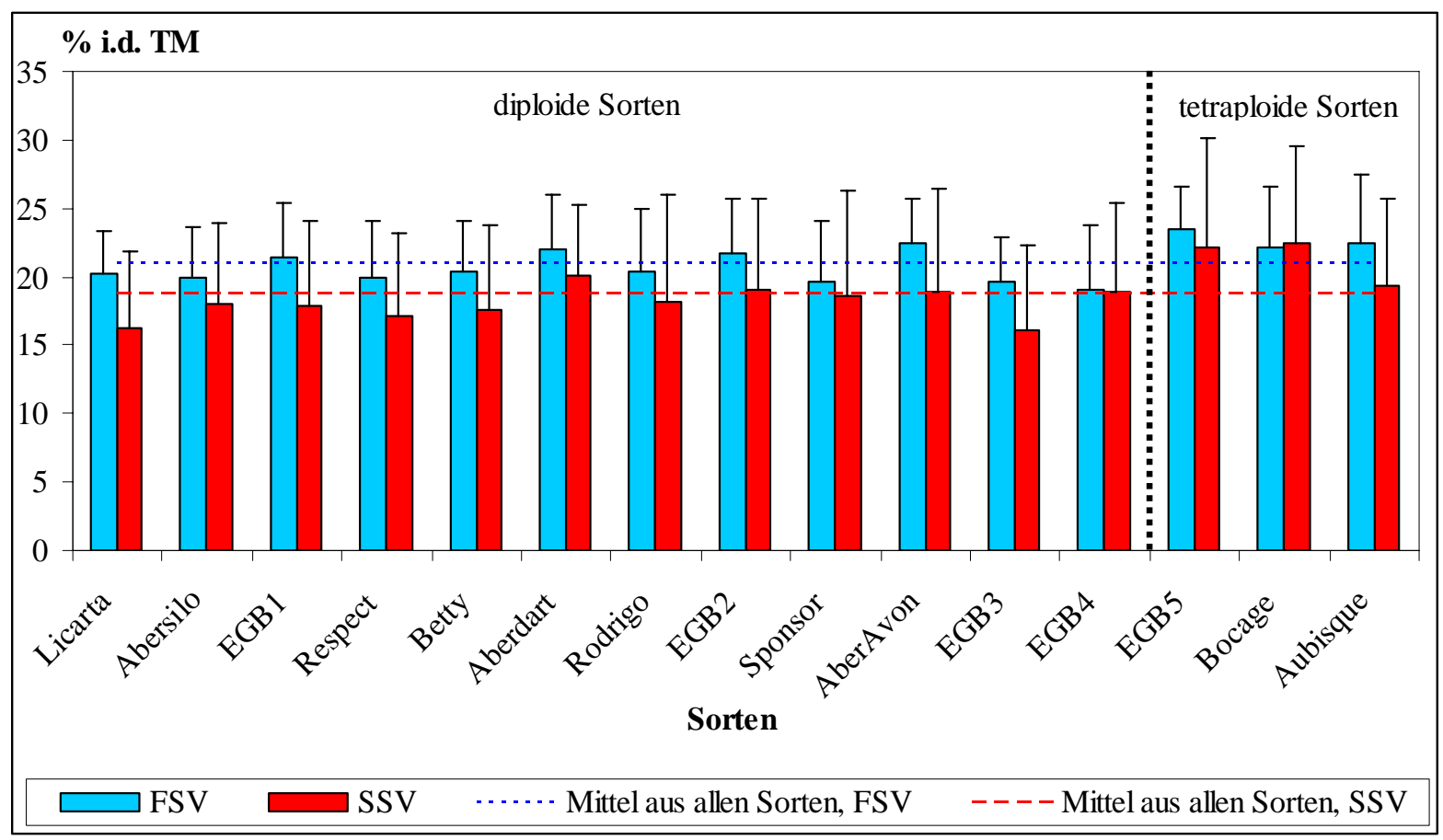

Abb.17: Wasserlösliche Kohlenhydrate (\% i.d. TM) in tetraploiden und diploiden Sorten des Deutschen Weidelgrases, Mittelwert aus den beiden Versuchsstandorten BS und HS und den beiden Versuchsjahren 1HVJ und 2HVJ im 1. Schnitt bei frühem und spätem Schnittzeitpunkt.

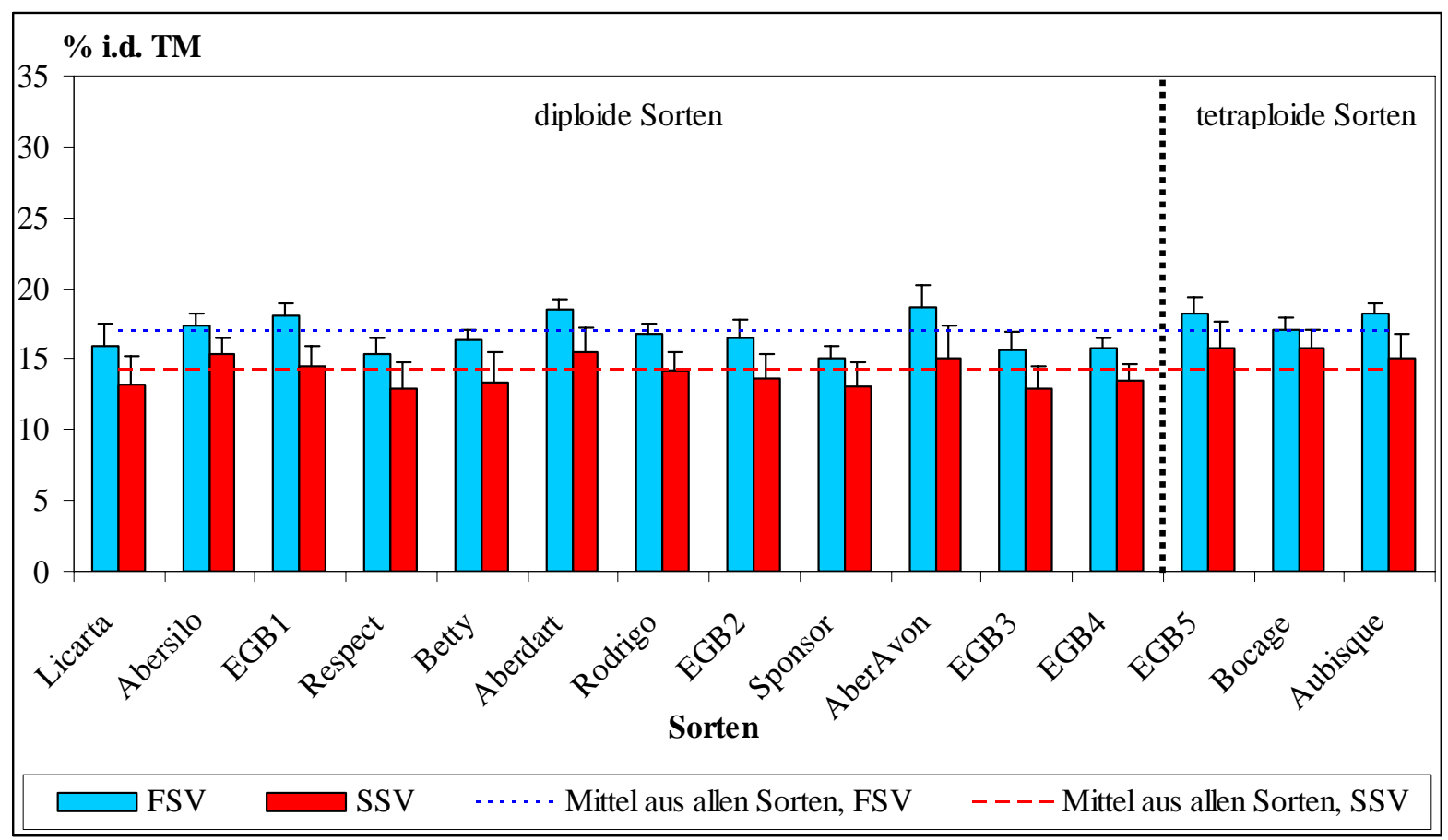

Abb. 18: Wasserlösliche Kohlenhydrate (\% i.d. TM) in tetraploiden und diploiden Sorten des Deutschen Weidelgrases, Mittelwert aus den beiden Versuchsstandorten BS und HS und den beiden Versuchsjahren 1HVJ und 2HVJ im Jahresmittel bei frühem und spätem Schnittzeitpunkt. 


\section{WIK-Gehalt im Verlauf der Vegetationsperiode}

Vom 1. zum 2. Schnitt fielen die WlK-Gehalte in der FSV im Sortenmittel sehr stark ab, vom 2. zum 4. Schnitt fielen sie leicht ab, vom 4. zum 5. Schnitt stiegen sie dagegen wieder an. In der SSV fielen sie vom 1. zum 3. Schnitt sehr stark ab und auch vom 3. zum 5. stiegen sie wieder ab, d.h, im Verlauf der gesamten Vegetationsperiode zeigten die WlK-Gehalte ein Frühjahrmaximum, eine Sommerdepression und einen Anstieg zum Herbst (Abb. 19).

Die WlK-Gehalte der FSV waren im Mittel der beiden HVJ im Verlauf der Vegetationsperiode höher als der SSV.

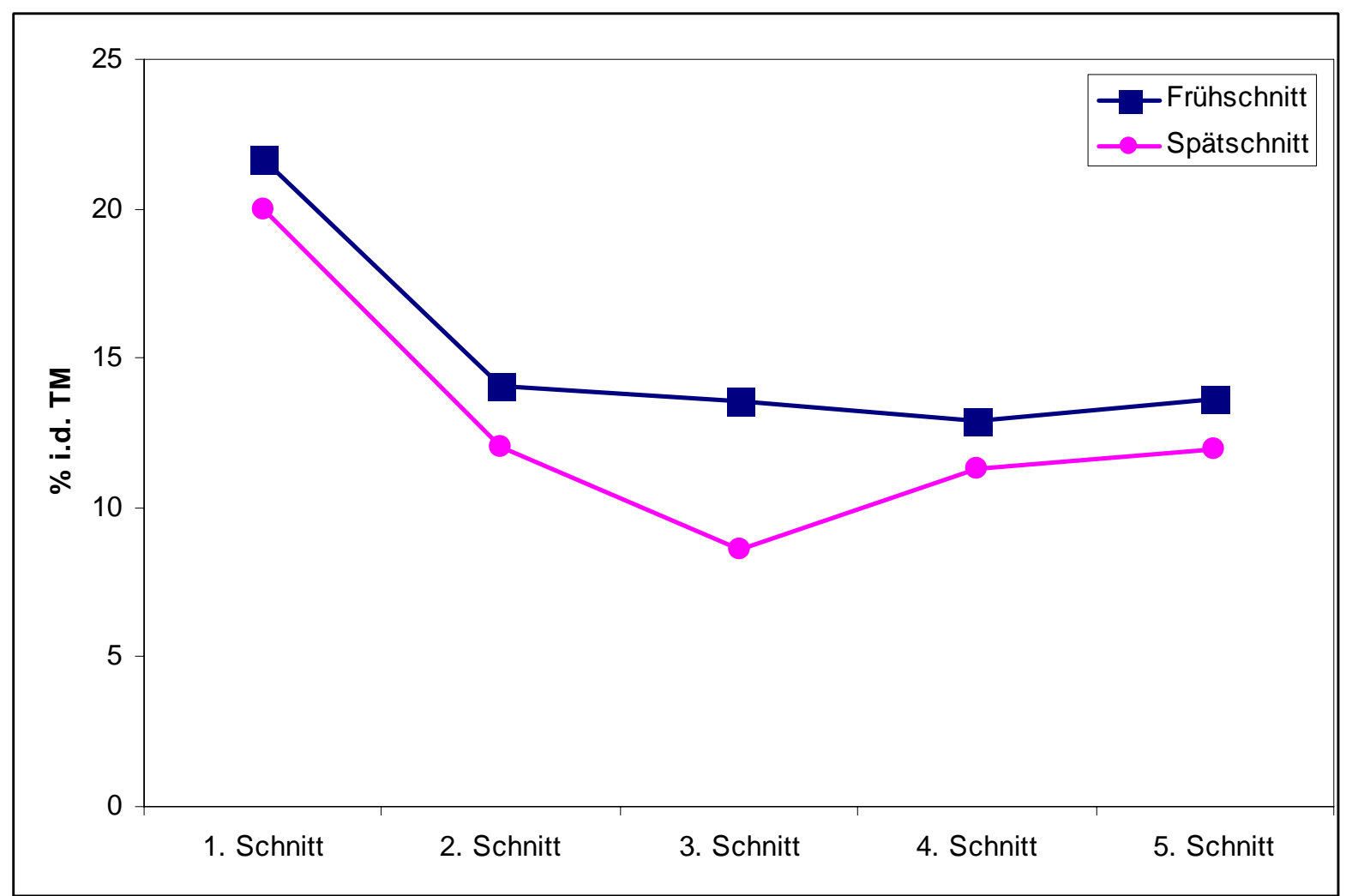

Abb. 19: Wasserlösliche Kohlenhydrate (\% i.d. TM) im Verlauf der Vegetationsperiode (Mittel aus allen 20 Sorten und den beiden Versuchsjahren 1HVJ und 2HVJ) an Versuchsstandort BS. 


\section{Fruktangehalt}

Die Sorten des Deutsches Weidelgrases hatten im 1HVJ einen höheren Fruktangehalt (\% in den WlK) als die Sorten des Wiesenschwingelgrases, des Wiesenschweidelgrases, Knaulgrases und Wiesenlieschgrases (Abb. 20). Im 2HVJ lag der Fruktangehalt (\% in den WlK) signifikant höher als im 1HVJ bei allen Gräserarten und nahm in der Reihenfolge Deutsches Weidelgras, Knaulgras, Wiesenschweidelgras, Wiesenschwingelgras und Wiesenlieschgras ab (Abb. 20).

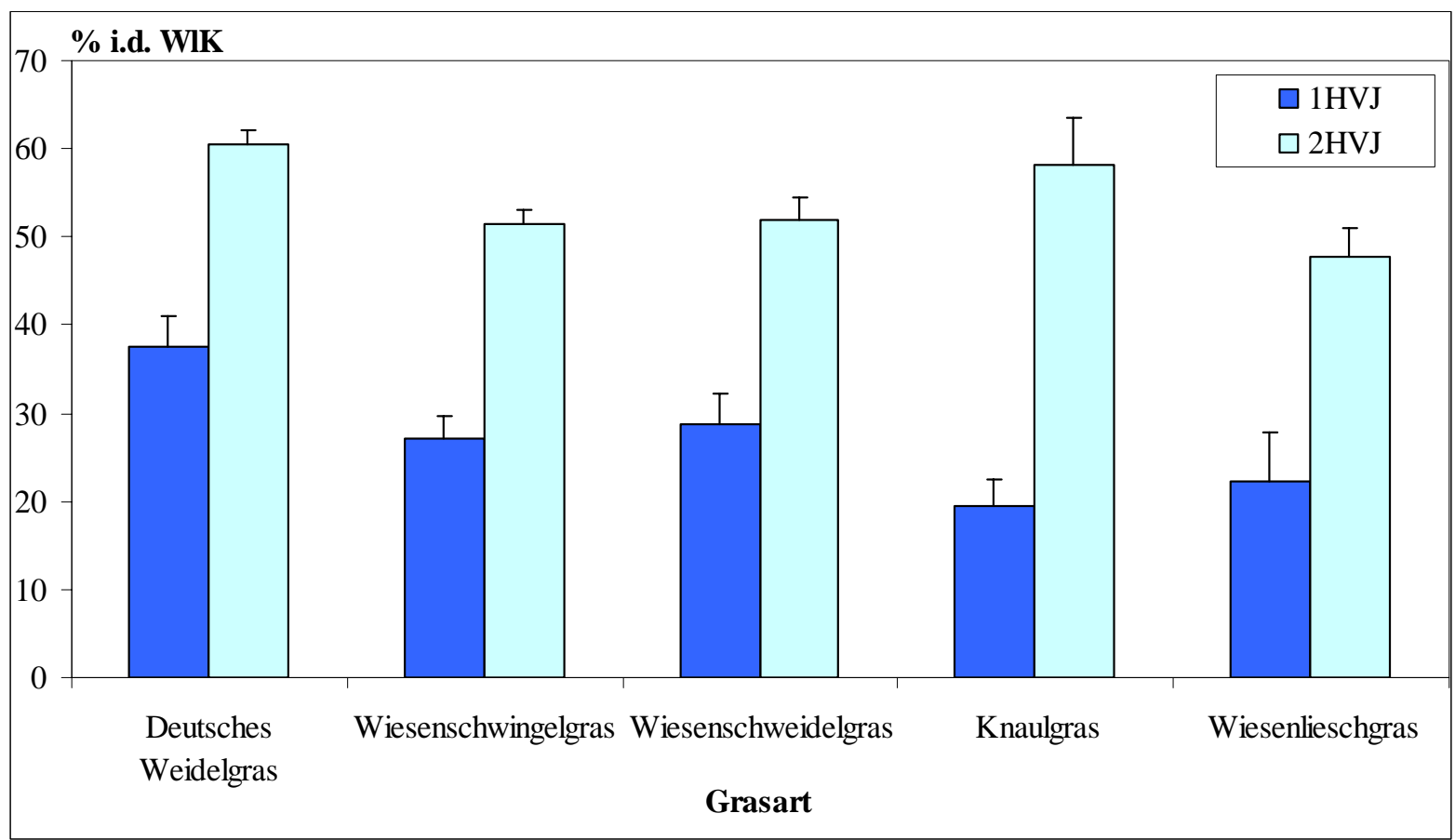

Abb. 20: Fruktangehalt (\% i. d. WlK) in verschiedenen Gräserarten des Grünlandes (Mittel aus den beiden Versuchsstandorten BS und HS und der FSV und der SSV) in den Versuchsjahren $1 \mathrm{HVJ}$ und $2 \mathrm{HVJ}$.

Im Verlauf der Vegetationsperiode fiel der Fruktangehalt (\% i.d. WlK) in der FSV und in der SSV vom 1. zum 3. Schnitt ab, vom 3. zum 4. Schnitt stieg er an und vom 4. zum 5. Schnitt stieg er wieder ab (Abb. 21)

Im 1. Schnitt lag der Fruktangehalt (\% i.d. WlK) in der SSV höher als in der FSV, in den folgenden Schnitten (2. bis 5. Schnitt) lag er in der FSV höher als SSV (Abb. 21). 


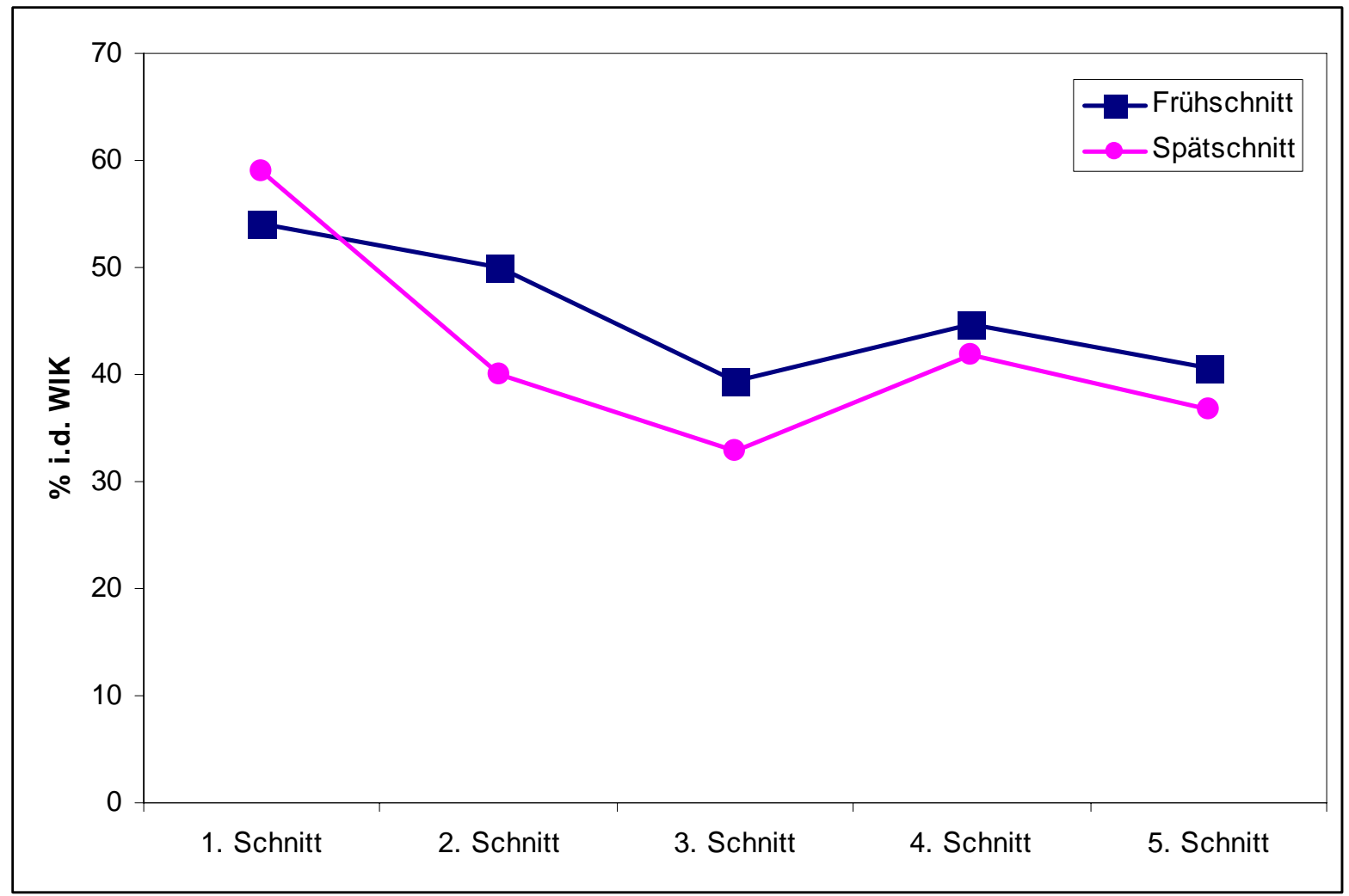

Abb. 21: Fruktangehalt (\% i. d. WlK) im Verlauf der Vegetationsperiode (Mittel aus allen 20 Sorten und den beiden Versuchsjahren 1HVJ und 2HVJ) am Versuchsstandort BS. 


\subsubsection{Verdaulichkeit (Enzymlösliche organischen Substanz)}

Am Versuchsstandort BS lag die Enzymlösliche organischer Substanz (ELOS) im Mittel der Sorten im 1. Schnitt der FSV im 2HVJ (2007) mit 69,1 \% i.d. TM signifikant höher als im 1HVJ (2006) mit 56,9 \% i.d. TM. Dagegen lag die ELOS in der SSV im 1HVJ mit 53,4 \% i.d. TM über dem Ergebnis des $2 \mathrm{HVJ}$ mit 50,2 \% i.d. TM. Auch im Jahresmittel lag die ELOS im Mittel der Sorten in der FSV im 2HVJ mit 69,7 \% i.d. TM deutlich über dem Ergebnis des 1HVJ mit 60,4 \% i.d. TM und in der SSV im $2 \mathrm{HVJ}$ mit 65,5 \% i.d. TM auch über dem Ergebnis des 1HVJ mit 60,1 \% i.d. TM (Tab. 21).

Am Versuchsstandort HS wiesen die ELOS im Mittel der Sorten im $\underline{1 . S c h n i t t}$ der FSV keine signifikanten Unterschiede zwischen 1HVJ (2006) und 2HVJ (2007) auf. In der SSV lag die ELOS im 1HVJ mit 52,1 \% i.d. TM über dem Ergebnis des 2HVJ mit 50,9 \% i.d. TM.

Im Jahresmittel lag die ELOS im Mittel der Sorten in der FSV im 2HVJ mit 68,4 \% i.d. TM deutlich über dem Ergebnis des 1HVJ mit 62 \% i.d. TM und in der SSV im 2HVJ mit 64,9 \% i.d. TM auch über dem Ergebnis des 1HVJ mit 57,4 \% i.d. TM (Tab. 21).

Im Mittel der beiden Versuchsstandorten (BS und HS) und im Mittel der Sorten im Jahresmittel der FSV lagen die ELOS im 2HVJ mit 69,1 \% i.d. TM deutlich über dem Ergebnis des 1HVJ mit 61,2 \% i.d. TM. Auch lagen die ELOS im Mittel der Sorten im Jahresmittel der SSV im 2HVJ mit 65,2 \% i.d. TM deutlich über dem Ergebnis des 1HVJ mit 58,8 \% i.d. TM.

Tab. 21: Versuchsjahresvergleich (2006/2007) für Enzymlösliche organischer Substanz (\% i.d. TM). Mittelwert aus allen Sorten im 1. Schnitt und im Jahresertrag.

\begin{tabular}{|c|c|c|c|c|c|c|c|c|c|c|c|c|}
\hline \multicolumn{13}{|l|}{ 1. Schnitt } \\
\hline \multirow{3}{*}{ Versuchsjahr } & \multicolumn{6}{|c|}{ BS } & \multicolumn{6}{|c|}{ HS } \\
\hline & \multicolumn{3}{|c|}{ FSV } & \multicolumn{3}{|c|}{ SSV } & \multicolumn{3}{|c|}{ FSV } & \multicolumn{3}{|c|}{ SSV } \\
\hline & Mw & & SD & Mw & & SD & Mw & & SD & Mw & & SD \\
\hline 2006 & 56,9 & $\mathrm{~b}$ & 9,2 & 53,4 & $\mathrm{a}$ & 9,3 & 62,5 & $\mathrm{a}$ & 5,8 & 52,1 & $\mathrm{a}$ & 7,3 \\
\hline 2007 & 69,1 & $\mathrm{a}$ & 6,9 & 50,2 & $\mathrm{~b}$ & 7,4 & 62,7 & $\mathrm{a}$ & 5,6 & 50,9 & $\mathrm{~b}$ & 6,5 \\
\hline \multicolumn{13}{|l|}{ Jahresmittel } \\
\hline 2006 & 60,4 & $\mathrm{~b}$ & 4,0 & 60,1 & $\mathrm{~b}$ & 3,6 & 62,0 & $\mathrm{~b}$ & 2,9 & 57,4 & $\mathrm{~b}$ & 3,3 \\
\hline 2007 & 69,7 & $\mathrm{a}$ & 4,2 & 65,5 & $\mathrm{a}$ & 4,2 & 68,4 & $\mathrm{a}$ & 4,3 & 64,9 & $\mathrm{a}$ & 3,9 \\
\hline
\end{tabular}

Signifikante Unterschiede gelten für Werte in derselben Spalten mit unterschiedlichen Buchstaben bei $\mathrm{P}<0,05$, $\mathrm{SD}=$ Standardabweichung, $\mathrm{Mw}=$ Mittelwert aus allen 20 Sorten und 3 Wiederholungen.

Am Versuchsstandort BS war die Verdaulichkeit (ELOS) im Mittel der Sorten im 1. Schnitt des 1HVJ und des 2HVJ bei der FSV höher als in der SSV. Im Jahresmittel wies die ELOS im Mittel der Sorten im 1HVJ keine signifikanten Unterschiede zwischen der FSV und der SSV auf. Im 2HVJ war die ELOS bei der FSV höher als bei der SSV (Tab. 22). 
Am Versuchsstandort HS lag ELOS im Mittel der Sorten im 1. Schnitt und im Jahresmittel des $1 \mathrm{HVJ}$ und des $2 \mathrm{HVJ}$ bei der FSV über dem Ergebnis der SSV (Tab. 22).

Im Mittel der beiden Versuchjahre (1HVJ und 2HVJ) und der beiden Versuchsstandorten (BS und HS) waren die ELOS im Mittel der Sorten im Jahresmittel bei der FSV (65,1 \% i.d. TM) deutlich höher als in der SSV (62,0 \% i.d. TM).

Tab. 22: Schnittzeitpunktvergleich (FSV/SSV) für Enzymlösliche organischer Substanz (\% i.d. TM). Mittelwert aus allen Sorten im 1. Schnitt und im Jahresmittel.

\begin{tabular}{|c|c|c|c|c|c|c|c|c|c|c|c|c|}
\hline \multicolumn{13}{|l|}{ 1. Schnitt } \\
\hline \multirow{3}{*}{ Schnittzeitpunkt } & \multicolumn{6}{|c|}{ BS } & \multicolumn{6}{|c|}{ HS } \\
\hline & \multicolumn{3}{|c|}{$1 \mathrm{HVJ}$} & \multicolumn{3}{|c|}{$2 \mathrm{HVJ}$} & \multicolumn{3}{|c|}{$1 \mathrm{HVJ}$} & \multicolumn{3}{|c|}{$2 \mathrm{HVJ}$} \\
\hline & $\mathrm{Mw}$ & & $\mathrm{SD}$ & Mw & & SD & $\mathrm{Mw}$ & & $\mathrm{SD}$ & Mw & & $\mathrm{SD}$ \\
\hline FSV & 56,9 & $\mathrm{a}$ & 9,2 & 69,1 & $\mathrm{a}$ & 6,8 & 62,5 & $\mathrm{a}$ & 5,8 & 62,7 & $\mathrm{a}$ & 5,6 \\
\hline SSV & 53,3 & $\mathrm{~b}$ & 9,3 & 50,2 & $\mathrm{~b}$ & 7,4 & 52,1 & $\mathrm{~b}$ & 7,3 & 51,0 & $\mathrm{~b}$ & 6,5 \\
\hline \multicolumn{13}{|l|}{ Jahresmittel } \\
\hline FSV & 60,4 & $\mathrm{a}$ & 4,0 & 69,7 & $\mathrm{a}$ & 4,2 & 62,0 & $\mathrm{a}$ & 2,9 & 68,4 & $\mathrm{a}$ & 4,3 \\
\hline SSV & 60,1 & $\mathrm{a}$ & 3,6 & 65,5 & $\mathrm{~b}$ & 4,2 & 53,4 & $\mathrm{~b}$ & 3,3 & 64,9 & $\mathrm{~b}$ & 3,9 \\
\hline
\end{tabular}

Signifikante Unterschiede gelten für Werte in derselben Spalte mit unterschiedlichen Buchstaben bei $\mathrm{P}<0,05$, $\mathrm{SD}=$ Standardabweichung, $\mathrm{Mw}=$ Mittelwert aus allen 20 Sorten und 3 Wiederholungen .

Die ELOS war in der FSV des 1HVJ am Versuchsstandort HS höher als am Versuchsstandort BS. Dagegen war sie in der SSV des 1HVJ und in der FSV des 2HVJ am Versuchsstandort BS höher als am Versuchsstandort HS. In der SSV des 2HVJ wies die ELOS keine signifikanten Unterschiede zwischen den Versuchsstandorten auf (Tab. 23).

Im Mittel der beiden Versuchsjahren (1HVJ und 2HVJ) wies die ELOS im 1. Schnitt der FSV keine signifikanten Unterschiede zwischen den Versuchsstandorten (BS und HS) (63,0 bzw. 62,6 \% i.d. TM) auf. Auch wies die ELOS in der SSV im 1. Schnitt der FSV keine signifikanten Unterschiede zwischen den Versuchsstandorten (BS und HS) (51,8 bzw. 51,5 \% i.d. TM) auf. 
Tab. 23: Enzymlösliche organischer Substanz (\% i.d. TM) an beiden Versuchsstandorte (BS und HS) im 1. Schnitt.

\begin{tabular}{|c|c|c|c|c|c|c|c|c|c|c|c|c|}
\hline \multirow{3}{*}{ Standort } & \multicolumn{6}{|c|}{$1 \mathrm{HVJ}$} & \multicolumn{6}{|c|}{$2 \mathrm{HVJ}$} \\
\hline & \multicolumn{3}{|c|}{ FSV } & \multicolumn{3}{|c|}{ SSV } & \multicolumn{3}{|c|}{ FSV } & \multicolumn{3}{|c|}{ SSV } \\
\hline & Mw & & SD & Mw & & SD & Mw & & SD & Mw & & SD \\
\hline $\mathrm{BS}$ & 56,8 & $\mathrm{~b}$ & 9,5 & 53,4 & $\mathrm{a}$ & 9,3 & 69,1 & $\mathrm{a}$ & 6,8 & 50,2 & $\mathrm{a}$ & 7,4 \\
\hline HS & 63,0 & $\mathrm{a}$ & 5,4 & 52,1 & b & 7,3 & 62,7 & b & 5,6 & 50,9 & $\mathrm{a}$ & 6,5 \\
\hline
\end{tabular}

Signifikante Unterschiede gelten für Werte in derselben Spalte mit unterschiedlichen Buchstaben bei $\mathrm{P}<0,05$, SD = Standardabweichung, Mw = Mittelwert aus allen 20 Sorten und 3 Wiederholungen .

Tabellen 24 und 25 zeigen die Unterschiede zwischen den Sorten in den Reifegruppen (früh, mittel und spät).

Reifegruppe „früh“:

Am Versuchsstandort BS im Jahresmittel der FSV und im 1. Schnitt und im Jahresmittel der SSV des 1HVJ sowie im 1. Schnitt und im Jahresmittel der FSV und der SSV des 2HVJ wies die ELOS keine signifikanten Unterschiede zwischen den Sorten (Licarta, Abersilo und EGB1) in der Reifegruppe „früh“ auf. Im 1. Schnitt der FSV des 1HVJ war die ELOS der Sorte EGB1 (59,1 \% i.d. TM) höher als die ELOS der Sorte Abersilo (52,2 \% i.d. TM) (Tab. 24).

Am Versuchsstandort HS wies die ELOS im 1. Schnitt und im Jahresmittel der FSV und der SSV des 1HVJ und im 1. Schnitt und im Jahresmittel der FSV sowie 1. Schnitt der SSV des 2HVJ keine signifikanten Unterschiede zwischen den Sorten in der Reifegruppe „früh“ auf. Im Jahresmittel der SSV des 2HVJ war die ELOS der Sorte EGB1 (66,4 \% i.d. TM) höher als die ELOS der Sorte Licarta (63 \% i.d. TM) (Tab. 25).

Im Mittel der beiden Versuchsjahren (1HVJ und 2HVJ) und der beiden Versuchsstandorten (BS und HS) wies die ELOS im Jahresmittel der FSV und der SSV keine signifikanten Unterschiede zwischen den Sorten (Licarta, Abersilo und EGB1) in der Reifegruppe „früh“ auf (Tab. 26).

\section{Reifegruppe „mittel“:}

Am Versuchsstandort BS wies die ELOS im 1. Schnitt der FSV und der SSV sowie im Jahresmittel der SSV des 1HVJ keine signifikanten Unterschiede zwischen den Sorten (Respect, Betty, Aberdart, Rodrigo, EGB2 und Aubisque) in der Reifegruppe „mittel“ auf.

Im Jahresmittel der FSV des 1HVJ gab es signifikante Unterschiede zwischen den Sorten; die Sorte Aubisque hatte die höchsten ELOS (64,3 \% i.d. TM), die Sorte Respect wies die niedrigsten ELOS (60,5 \% i.d. TM) auf. Im 1. Schnitt und Jahresmittel der FSV des 2HVJ gab 
es signifikante Unterschiede zwischen den Sorten; die ELOS war in der Sorte Aubisque am höchsten, in der Sorte Respect am niedrigsten. Im 1. Schnitt und Jahresmittel der SSV des 2HVJ wies die ELOS keine signifikanten Unterschiede zwischen den Sorten auf (Tab.24).

Am Versuchsstandort HS wies die ELOS im 1. Schnitt und im Jahresmittel der FSV und der SSV des 1HVJ sowie im Jahresmittel der FSV und der SSV des 2HVJ keine signifikanten Unterschiede zwischen den Sorten in der Reifegruppe „mittel“ auf. Im 1. Schnitt der FSV des 2HVJ gab es signifikante Unterschiede zwischen den Sorten; die Sorte EGB2 hatte die höchsten ELOS (67,1 \% i.d. TM), die Sorte Betty den niedrigsten (58,9 \% i.d. TM).

Auch im 1. Schnitt der SSV des 2HVJ gab es signifikante Unterschiede zwischen den Sorten. Die Sorte EGB2 zeigte die höchsten ELOS (57,8 \% i.d. TM), die Sorte Respect die niedrigsten ELOS (47,6 \% i.d. TM) (Tab. 25).

Im Mittel der beiden Versuchsjahren (1HVJ und 2HVJ) und der beiden Versuchsstandorten (BS und HS) gab es im Jahresmittel der FSV signifikante Unterschiede zwischen den Sorten; die ELOS war in der Sorte Aubisque am höchsten (68,1 \% i.d. TM), in der Sorte Betty am niedrigsten (65,5 \% i.d. TM). Im Jahresmittel der SSV wies die ELOS keine signifikanten Unterschiede zwischen den Sorten (Respect, Betty, Aberdart, Rodrigo, EGB2 und Aubisque) in der Reifegruppe „mittel“ auf (Tab. 26).

\section{Reifegruppe „spät““:}

Am $\underline{\text { Versuchsstandort BS }}$ wies die ELOS im 1. Schnitt und im Jahresmittel der FSV des 1HVJ signifikanten Unterschiede zwischen den Sorten (Sponsor, AberAvon, EGB3, EGB4, EGB5 und Bocage) in der Reifegruppe „spät“ auf; die Sorte EGB5 hatte den höchsten ELOS, die Sorte Sponsor die niedrigsten. Im 1. Schnitt der SSV des 1HVJ gab es signifikante Unterschiede zwischen den Sorten; die Sorte EGB5 hatte die höchsten ELOS (69,3 \% i.d. TM), die Sorte Sponsor die niedrigsten (56,9 \% i.d. TM). Im Jahresmittel der SSV des 1HVJ gab es signifikante Unterschiede zwischen den Sorten. Hier zeigte auch die Sorte EGB5 die höchsten ELOS (65,5 \% i.d. TM), die Sorten EGB3 die niedrigsten (60,8 \% i.d. TM).

Im 1. Schnitt der FSV des 2HVJ wies die ELOS keine signifikanten Unterschiede zwischen den Sorten in der Reifegruppe „spät“ auf. Im Jahresmittel der FSV des 2HVJ sowie im 1. Schnitt und im Jahresmittel der SSV des 2HVJ gab es signifikante Unterschiede zwischen den Sorten; die Sorte EGB5 hatte den höchsten ELOS, die Sorte Sponsor die niedrigsten (Tab. 24). 
Am Versuchsstandort HS wies die ELOS im 1. Schnitt der FSV des 1HVJ keine signifikante Unterschiede zwischen den Sorten in der Reifegruppe „spät“ auf. Im Jahresmittel der FSV des 1HVJ wies die ELOS signifikante Unterschiede zwischen den Sorten auf, die Sorte EGB5 hatte die höchste ELOS (66,7 \% i.d. TM), die Sorte EGB4 die niedrigste (62,5 \% i.d. TM). Im 1. Schnitt und im Jahresmittel der SSV des 1HVJ gab es signifikante Unterschiede zwischen den Sorten. Hier zeigte auch die Sorte EGB5 die höchsten ELOS, die Sorte Sponsor die niedrigsten. Im 1. Schnitt und im Jahresmittel der FSV des 2HVJ wies die ELOS signifikante Unterschiede zwischen den Sorten auf; die Sorte EGB5 hatte die höchste ELOS, die Sorte Sponsor und die Sorte EGB3 die niedrigste. Auch im 1. Schnitt und im Jahresmittel der SSV des 2HVJ gab es signifikante Unterschiede zwischen den Sorten. Hier zeigte die Sorte Bocage die höchsten ELOS, die Sorten Sponsor die niedrigsten (Tab. 25).

Im Mittel der beiden Versuchsjahren (1HVJ und 2HVJ) und der beiden Versuchsstandorten (BS und HS) wies die ELOS im Jahresmittel der FSV signifikante Unterschiede zwischen den Sorten (Sponsor, AberAvon, EGB3, EGB4, EGB5 und Bocage) in der Reifegruppe „spät“ auf; die Sorte EGB5 hatte den höchsten ELOS (70,0 \% i.d. TM), die Sorte Sponsor die niedrigsten (65,9 \% i.d. TM). Auch im Jahresmittel der SSV wies die ELOS gab es signifikante Unterschiede zwischen den Sorten. Hier zeigte die Sorte EGB5 die höchsten ELOS (67,5 \% i.d. TM), die Sorten EGB3 die niedrigsten (63,1 \% i.d. TM) (Tab. 26). 
Tab. 24: Enzymlösliche organischer Substanz (\% i.d. TM) in Abhängigkeit von den Sortenreifengruppen (früh, mittel und spät) des Deutschen Weidelgrases am Versuchsstandort BS im 1HVJ (2006) und im 2HVJ (2007).

\begin{tabular}{|c|c|c|c|c|c|c|c|c|c|}
\hline \multicolumn{10}{|c|}{ 1HVJ (2006) } \\
\hline \multirow{3}{*}{\multicolumn{2}{|c|}{$\begin{array}{l}\text { Reifegruppe/ } \\
\text { Sorten }\end{array}$}} & \multicolumn{8}{|c|}{ Schnittzeitpunkt } \\
\hline & & \multicolumn{4}{|c|}{ FSV } & \multicolumn{4}{|c|}{ SSV } \\
\hline & & 1. Schnitt & SD & Jahresmittel & SD & 1. Schnitt & SD & Jahresmittel & SD \\
\hline \multirow{3}{*}{ früh } & Licarta & 54,9 a b & 2,7 & $61,0 \mathrm{a}$ & 0,6 & $51,1 \mathrm{a}$ & 2,3 & 60,8 a & 0,8 \\
\hline & Abersilo & $52,2 \mathrm{~b}$ & 1,0 & $61,3 \mathrm{a}$ & 1,0 & $50,1 \mathrm{a}$ & 3,6 & $61,4 \mathrm{a}$ & 0,6 \\
\hline & EGB1 & $59,1 \mathrm{a}$ & 1,5 & $63,1 \mathrm{a}$ & 0,8 & $54,6 \mathrm{a}$ & 1,7 & $62,2 \mathrm{a}$ & 0,8 \\
\hline \multirow{6}{*}{ mittel } & Respect & $58,4 \mathrm{a}$ & 2,1 & $60,5 \mathrm{~b}$ & 1,3 & $54,1 \mathrm{a}$ & 1,5 & $60,6 \mathrm{a}$ & 0,3 \\
\hline & Betty & 58,4 a & 3,2 & 61,6 a b & 1,8 & 54,5 a & 3,0 & 59,9 a & 2,2 \\
\hline & Aberdart & $59,6 \mathrm{a}$ & 1,2 & $63,0 \mathrm{a} b$ & 0,4 & $56,2 \mathrm{a}$ & 1,4 & $61,9 \mathrm{a}$ & 0,3 \\
\hline & Rodrigo & $60,8 \mathrm{a}$ & 1,2 & $61,4 \mathrm{a} \mathrm{b}$ & 1,3 & $56,6 \mathrm{a}$ & 1,9 & $61,5 \mathrm{a}$ & 0,8 \\
\hline & EGB2 & $63,9 a$ & 2,6 & $61,6 \mathrm{a} \mathrm{b}$ & 0,4 & $55,9 \mathrm{a}$ & 1,5 & $60,5 \mathrm{a}$ & 1,2 \\
\hline & Aubisque & 61,3 a & 1,7 & 64,3 a & 0,8 & $55,9 \mathrm{a}$ & 1,1 & 61,9 a & 2,3 \\
\hline \multirow{6}{*}{ spät } & Sponsor & $63,5 \mathrm{~b}$ & 2,8 & $61,0 \mathrm{c}$ & 0,8 & $56,9 \mathrm{c}$ & 1,5 & $61,9 \mathrm{~b} \mathrm{c}$ & 1,7 \\
\hline & AberAvon & $64,7 \mathrm{~b}$ & 0,6 & 63,0 a b c & 0,5 & $60,8 \mathrm{~b} \mathrm{c}$ & 0,9 & 63,1 a b c & 0,7 \\
\hline & EGB3 & $63,6 \mathrm{~b}$ & 1,1 & $61,8 \mathrm{~b} \mathrm{c}$ & 0,7 & $60,8 \mathrm{c}$ & 3,1 & $60,8 \mathrm{c}$ & 1,4 \\
\hline & EGB4 & $65,3 \mathrm{a} \mathrm{b}$ & 1,4 & $62,4 \mathrm{a}$ b c & 0,9 & $60,9 \mathrm{~b} \mathrm{c}$ & 3,3 & $61,4 \mathrm{c}$ & 0,7 \\
\hline & EGB5 & 69,6 a & 1,9 & $64,8 \mathrm{a}$ & 1,8 & $69,3 \mathrm{a}$ & 1,2 & $65,5 \mathrm{a}$ & 0,8 \\
\hline & Bocage & $66,7 \mathrm{a} \mathrm{b}$ & 0,8 & $64,1 \mathrm{a} \mathrm{b}$ & 0,4 & $66,4 \mathrm{a} \mathrm{b}$ & 0,9 & $64,0 \mathrm{a} \mathrm{b}$ & 0,5 \\
\hline \multicolumn{10}{|c|}{$2 \mathrm{HVJ}(2007)$} \\
\hline \multirow{3}{*}{ früh } & Licarta & $71,0 \mathrm{a}$ & 3,2 & $70,5 \mathrm{a}$ & 0,8 & $47,8 \mathrm{a}$ & 1,1 & $65,8 \mathrm{a}$ & 1,4 \\
\hline & Abersilo & $68,3 \mathrm{a}$ & 1,7 & $71,5 \mathrm{a}$ & 1,1 & $45,2 \mathrm{a}$ & 3,8 & 67,0 a & 0,9 \\
\hline & EGB1 & $68,5 \mathrm{a}$ & 0,2 & $70,8 \mathrm{a}$ & 1,0 & $48,5 \mathrm{a}$ & 3,6 & $66,7 \mathrm{a}$ & 0,6 \\
\hline \multirow{6}{*}{ mittel } & Respect & $69,1 \mathrm{~b}$ & 1,7 & $70,2 \mathrm{~b}$ & 0,9 & $46,8 \mathrm{a}$ & 1,8 & $67,2 \mathrm{a}$ & 1,1 \\
\hline & Betty & $69,2 \mathrm{~b}$ & 1,6 & $71,0 \mathrm{a} \mathrm{b}$ & 2,1 & $47,4 \mathrm{a}$ & 1,3 & $66,3 \mathrm{a}$ & 0,9 \\
\hline & Aberdart & $72,1 \mathrm{a} b$ & 1,0 & $72,6 \mathrm{a} b$ & 1,0 & $49,1 \mathrm{a}$ & 2,7 & $67,0 \mathrm{a}$ & 2,4 \\
\hline & Rodrigo & $67,3 \mathrm{~b}$ & 0,5 & $70,7 \mathrm{~b}$ & 0,3 & $49,3 \mathrm{a}$ & 0,8 & $67,0 \mathrm{a}$ & 0,6 \\
\hline & EGB2 & $72,2 \mathrm{a} \mathrm{b}$ & 0,1 & $70,6 \mathrm{~b}$ & 0,9 & $52,9 \mathrm{a}$ & 0,9 & $65,4 \mathrm{a}$ & 1,4 \\
\hline & Aubisque & $76,6 \mathrm{a}$ & 1,2 & $74,0 \mathrm{a}$ & 0,5 & $50,7 \mathrm{a}$ & 1,4 & $67,3 \mathrm{a}$ & 2,0 \\
\hline \multirow{6}{*}{ spät } & Sponsor & $71,6 \mathrm{a}$ & 1,3 & $69,9 \mathrm{~b}$ & 0,4 & $50,4 \mathrm{c}$ & 1,2 & $66,6 \mathrm{~b}$ & 1,3 \\
\hline & AberAvon & $72,4 \mathrm{a}$ & 4,0 & $72,1 \mathrm{~b}$ & 0,5 & $54,1 \mathrm{c}$ & 1,4 & $68,2 \mathrm{~b}$ & 1,8 \\
\hline & EGB3 & $72,5 \mathrm{a}$ & 2,9 & $70,0 \mathrm{~b}$ & 1,6 & $53,8 \mathrm{c}$ & 1,5 & $65,6 \mathrm{~b}$ & 0,2 \\
\hline & EGB4 & $75,4 \mathrm{a}$ & 1,7 & $71,0 \mathrm{~b}$ & 0,5 & $58,3 \mathrm{~b}$ & 2,0 & $67,5 \mathrm{~b}$ & 0,6 \\
\hline & EGB5 & $78,2 \mathrm{a}$ & 2,0 & $75,0 \mathrm{a}$ & 0,8 & $67,0 \mathrm{a}$ & 1,3 & $72,3 \mathrm{a}$ & 0,7 \\
\hline & Bocage & 77,9 a & 1,7 & 74,5 a & 0,3 & $65,4 \mathrm{a}$ & 4,1 & 71,3 a & 0,3 \\
\hline
\end{tabular}

Signifikante Unterschiede gelten für gruppierte Zellen mit unterschiedlichen Buchstaben $(\mathrm{P}<0,05), \mathrm{SD}=$ Standardabweichung. 
Tab. 25: Enzymlösliche organischer Substanz (\% i.d. TM) in Abhängigkeit von den Sortenreifengruppen (früh, mittel und spät) des Deutschen Weidelgrases am Versuchsstandort HS im 1HVJ (2006) und im 2HVJ (2007).

\begin{tabular}{|c|c|c|c|c|c|c|c|c|c|}
\hline \multicolumn{10}{|c|}{ 1HVJ (2006) } \\
\hline \multirow{3}{*}{\multicolumn{2}{|c|}{$\begin{array}{l}\text { Reifegruppe/ } \\
\text { Sorten }\end{array}$}} & \multicolumn{8}{|c|}{ Schnittzeitpunkt } \\
\hline & & \multicolumn{4}{|c|}{ FSV } & \multicolumn{4}{|c|}{ SSV } \\
\hline & & 1. Schnitt & $\mathrm{SD}$ & Jahresmittel & SD & 1. Schnitt & SD & Jahresmittel & $\mathrm{SD}$ \\
\hline \multirow{3}{*}{ früh } & Licarta & $62,4 \mathrm{a}$ & 1,8 & 61,9 a & 1,0 & $48,6 \mathrm{a}$ & 2,6 & 55,9 a & 2,0 \\
\hline & Abersilo & $59,5 \mathrm{a}$ & 2,5 & $61,7 \mathrm{a}$ & 0,8 & $49,0 \mathrm{a}$ & 3,4 & $57,4 \mathrm{a}$ & 1,7 \\
\hline & EGB1 & $59,4 \mathrm{a}$ & 4,1 & $63,2 \mathrm{a}$ & 1,2 & $51,3 \mathrm{a}$ & 2,8 & $57,0 \mathrm{a}$ & 1,7 \\
\hline \multirow{6}{*}{ mittel } & Respect & $62,2 \mathrm{a}$ & 4,0 & $63,0 \mathrm{a}$ & 1,6 & $52,2 \mathrm{a}$ & 1,6 & $56,8 \mathrm{a}$ & 0,6 \\
\hline & Betty & $60,4 \mathrm{a}$ & 4,0 & $60,6 \mathrm{a}$ & 1,3 & $51,9 a$ & 3,2 & $57,0 \mathrm{a}$ & 1,0 \\
\hline & Aberdart & $59,5 \mathrm{a}$ & 4,6 & $62,7 \mathrm{a}$ & 1,5 & $52,3 \mathrm{a}$ & 3,5 & $58,6 \mathrm{a}$ & 1,0 \\
\hline & Rodrigo & $61,0 \mathrm{a}$ & 1,5 & $62,8 \mathrm{a}$ & 0,8 & $50,8 \mathrm{a}$ & 1,7 & $58,7 \mathrm{a}$ & 1,8 \\
\hline & EGB2 & $67,1 \mathrm{a}$ & 0,3 & $63,1 \mathrm{a}$ & 0,6 & $58,0 \mathrm{a}$ & 1,0 & $58,5 \mathrm{a}$ & 0,6 \\
\hline & Aubisque & $61,6 \mathrm{a}$ & 1,0 & $62,8 \mathrm{a}$ & 0,8 & $51,3 \mathrm{a}$ & 3,4 & $58,5 \mathrm{a}$ & 0,4 \\
\hline \multirow{6}{*}{ spät } & Sponsor & $67,2 \mathrm{a}$ & 1,9 & $62,7 \mathrm{~b}$ & 0,6 & $58,8 \mathrm{~b}$ & 1,3 & $58,9 \mathrm{~b}$ & 0,2 \\
\hline & AberAvon & $65,6 \mathrm{a}$ & 2,5 & 64,9 a b & 0,7 & 59,7 a b & 2,4 & $59,6 \mathrm{~b}$ & 1,4 \\
\hline & EGB3 & $69,1 \mathrm{a}$ & 1,8 & 63,7 a b & 0,4 & $57,3 \mathrm{~b}$ & 1,8 & $59,5 \mathrm{~b}$ & 0,6 \\
\hline & EGB4 & $63,1 \mathrm{a}$ & 8,9 & $62,5 \mathrm{~b}$ & 3,0 & $61,5 \mathrm{a} \mathrm{b}$ & 0,9 & $60,5 \mathrm{a} \mathrm{b}$ & 1,7 \\
\hline & EGB5 & $73,2 \mathrm{a}$ & 0,5 & $66,7 \mathrm{a}$ & 0,3 & $64,2 \mathrm{a}$ & 1,2 & $62,6 \mathrm{a}$ & 1,1 \\
\hline & Bocage & $71,0 \mathrm{a}$ & 1,0 & 64,8 a b & 0,6 & $61,5 \mathrm{a} \mathrm{b}$ & 2,4 & $61,2 \mathrm{ab}$ & 0,7 \\
\hline \multicolumn{10}{|c|}{ 2HVJ (2007) } \\
\hline \multirow{3}{*}{ früh } & Licarta & $61,9 \mathrm{a}$ & 1,2 & $68,6 \mathrm{a}$ & 2,2 & $45,0 \mathrm{a}$ & 2,1 & $63,0 \mathrm{~b}$ & 1,2 \\
\hline & Abersilo & $60,2 \mathrm{a}$ & 2,3 & $68,2 \mathrm{a}$ & 1,5 & $47,7 \mathrm{a}$ & 2,1 & 65,0 a b & 0,8 \\
\hline & EGB1 & $62,0 \mathrm{a}$ & 2,2 & $70,2 \mathrm{a}$ & 1,7 & $50,6 \mathrm{a}$ & 1,8 & $66,4 \mathrm{a}$ & 1,5 \\
\hline \multirow{6}{*}{ mittel } & Respect & 59,5 a b & 2,9 & $69,0 \mathrm{a}$ & 0,7 & $47,6 \mathrm{~b}$ & 2,1 & $64,8 \mathrm{a}$ & 1,8 \\
\hline & Betty & $58,9 \mathrm{~b}$ & 5,9 & $69,0 \mathrm{a}$ & 1,3 & $49,4 \mathrm{~b}$ & 2,0 & $65,0 \mathrm{a}$ & 1,4 \\
\hline & Aberdart & $64,1 \mathrm{a} b$ & 2,8 & $70,8 \mathrm{a}$ & 1,3 & $52,8 \mathrm{a} \mathrm{b}$ & 3,9 & $67,7 \mathrm{a}$ & 1,9 \\
\hline & Rodrigo & 64,0 a b & 2,4 & $71,6 \mathrm{a}$ & 0,5 & 50,6 a b & 1,5 & $66,6 \mathrm{a}$ & 0,5 \\
\hline & EGB2 & $67,1 \mathrm{a}$ & 2,1 & $70,4 \mathrm{a}$ & 1,5 & $57,8 \mathrm{a}$ & 5,5 & $67,3 \mathrm{a}$ & 0,8 \\
\hline & Aubisque & 63,9 a b & 1,6 & $71,2 \mathrm{a}$ & 2,0 & 50,7 a b & 4,1 & $66,4 \mathrm{a}$ & 1,1 \\
\hline \multirow{6}{*}{ spät } & Sponsor & $64,9 \mathrm{~b}$ & 2,3 & $69,8 \mathrm{~b}$ & 0,3 & $53,0 \mathrm{~b}$ & 1,2 & $66,3 \mathrm{c}$ & 0,2 \\
\hline & AberAvon & $67,2 \mathrm{a} b$ & 0,7 & $72,0 \mathrm{a} b$ & 0,4 & 55,5 a b & 2,9 & $67,8 \mathrm{~b} \mathrm{c}$ & 0,3 \\
\hline & EGB3 & $66,0 \mathrm{~b}$ & 1,2 & $69,7 \mathrm{~b}$ & 1,3 & $54,1 \mathrm{~b}$ & 1,7 & $66,6 \mathrm{c}$ & 1,0 \\
\hline & EGB4 & $67,4 \mathrm{a} b$ & 1,3 & $70,0 \mathrm{~b}$ & 1,1 & 58,7 a b & 4,7 & $67,4 \mathrm{c}$ & 0,1 \\
\hline & EGB5 & $71,1 \mathrm{a}$ & 1,5 & $73,5 \mathrm{a}$ & 0,7 & $61,2 \mathrm{a} \mathrm{b}$ & 2,0 & $69,6 \mathrm{a} \mathrm{b}$ & 1,3 \\
\hline & Bocage & $71,0 \mathrm{a}$ & 1,4 & 73,3 a & 1,0 & $62,6 \mathrm{a}$ & 1,5 & 69,9 a & 0,3 \\
\hline
\end{tabular}

Signifikante Unterschiede gelten für gruppierte Zellen mit unterschiedlichen Buchstaben $(\mathrm{P}<0,05)$, $\mathrm{SD}=$ Standardabweichung. 
Tab. 26: Enzymlösliche organischer Substanz (ELOS) (\% i.d. TM) in Abhängigkeit von den Sortenreifengruppen (früh, mittel und spät) des Deutschen Weidelgrases, im Mittel der beiden Versuchsjahren (1HVJ und 2HVJ) und der beiden Versuchsstandorten (BS und HS), im Jahresmittel.

\begin{tabular}{|c|c|c|c|c|c|c|c|}
\hline \multirow{3}{*}{\multicolumn{2}{|c|}{$\begin{array}{l}\text { Reifegruppe/ } \\
\text { Sorten }\end{array}$}} & \multicolumn{6}{|c|}{ Schnittzeitpunkt } \\
\hline & & \multicolumn{3}{|c|}{ FSV } & \multicolumn{3}{|c|}{ FSV } \\
\hline & & ELOS & & SD & ELOS & & SD \\
\hline \multirow{3}{*}{ früh } & Licarta & 65,5 & $\bar{a}$ & 0,87 & 61,3 & $a$ & $\overline{1,01}$ \\
\hline & Abersilo & 65,6 & $\mathrm{a}$ & 0,57 & 62,7 & $\mathrm{a}$ & 0,66 \\
\hline & EGB1 & 66,8 & $\mathrm{a}$ & 0,40 & 63,1 & $\mathrm{a}$ & 0,43 \\
\hline \multirow{6}{*}{ mittel } & Respect & 65,7 & $\mathrm{~b}$ & 1,10 & 62,4 & $\mathrm{a}$ & 0,83 \\
\hline & Betty & 65,5 & $\mathrm{~b}$ & 1,02 & 62,0 & $\mathrm{a}$ & 0,57 \\
\hline & Aberdart & 67,3 & $\mathrm{ab}$ & 0,50 & 63,8 & $\mathrm{a}$ & 1,17 \\
\hline & Rodrigo & 66,6 & $\mathrm{ab}$ & 0,20 & 63,4 & $\mathrm{a}$ & 0,35 \\
\hline & EGB2 & 66,4 & $\mathrm{ab}$ & 0,20 & 62,9 & $\mathrm{a}$ & 0,23 \\
\hline & Aubisque & 68,1 & $\mathrm{a}$ & 1,10 & 63,6 & $\mathrm{a}$ & 1,14 \\
\hline \multirow{6}{*}{ spät } & Sponsor & 65,9 & $\mathrm{~d}$ & 0,12 & 63,5 & bc & 0,78 \\
\hline & AberAvon & 68,0 & bc & 0,53 & 64,7 & $\mathrm{~b}$ & 0,61 \\
\hline & EGB3 & 66,3 & $\mathrm{~d}$ & 0,70 & 63,1 & C & 0,60 \\
\hline & EGB4 & 66,5 & $\mathrm{~cd}$ & 1,10 & 64,2 & bc & 0,43 \\
\hline & EGB5 & 70,0 & $\mathrm{a}$ & 0,64 & 67,5 & $\mathrm{a}$ & 0,23 \\
\hline & Bocage & 69,2 & $\mathrm{ab}$ & 0,12 & 66,6 & $\mathrm{a}$ & 0,36 \\
\hline
\end{tabular}

Signifikante Unterschiede gelten für gruppierte Zellen mit unterschiedlichen Buchstaben $(\mathrm{P}<0,05)$, $\mathrm{SD}=$ Standardabweichung. 


\section{Grasarten- und Sortenunterschiede}

Die Verdaulichkeit nahm in der Reihenfolge Deutsches Weidelgras, Wiesenschwingelgras, Wiesenschweidelgras, Knaulgras und Wiesenlieschgras ab (Abb. 22).

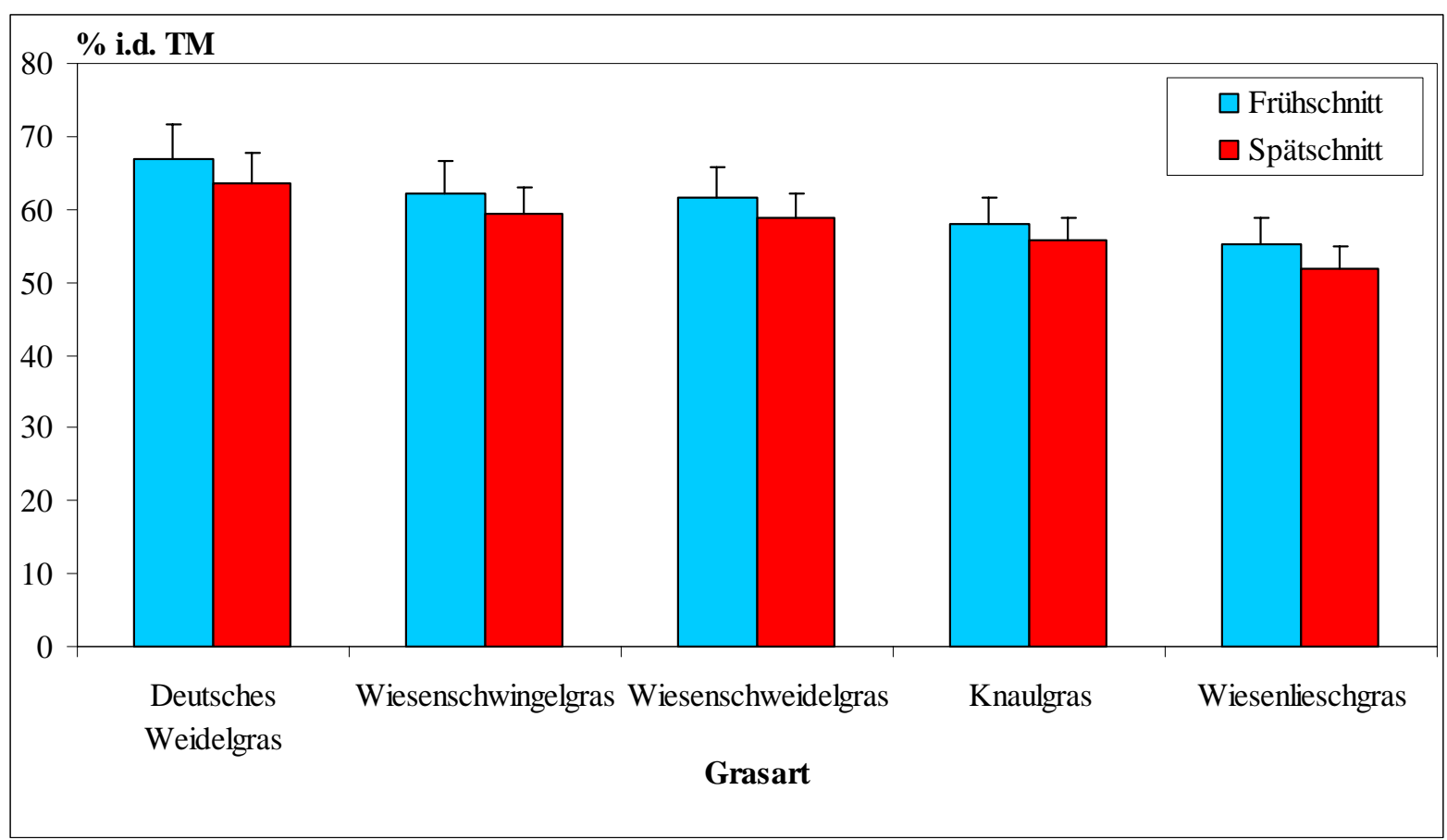

Abb. 22: Verdaulichkeit (\% i.d. TM) in verschiedenen Gräserarten des Grünlandes, Mittelwert aus den beiden Versuchsstandorten BS und HS und den beiden Versuchsjahren 1HVJ und 2HVJ bei frühem und spätem Schnittzeitpunkt.

Ein Blick auf die Abbildung 23 zeigt, dass Bei Deutschem Weidelgras nahm die Verdaulichkeit im 1. Schnitt deutlich von späten zu frühen Sorten ab.

Im Mittel der beiden Versuchsjahre (1HVJ und 2HVJ) und der beiden Versuchsstandorten (BS und HS) wiesen die Sorten AberAvon (68 für FSV, 64,7 für SSV \% i.d. TM) und Aberdart (67,3 für FSV, 63,8 für SSV \% i.d. TM) in der Diploidegruppe im Jahresmittel die höchsten Verdaulichkeit auf (Abb. 24).

Die Verdaulichkeit war bei den tetraploiden Sorten des Deutschen Weidelgrases (EGB5 und Bocage) höher als bei den diploiden Sorten in derselben Reifegruppe (Abb. 23). 


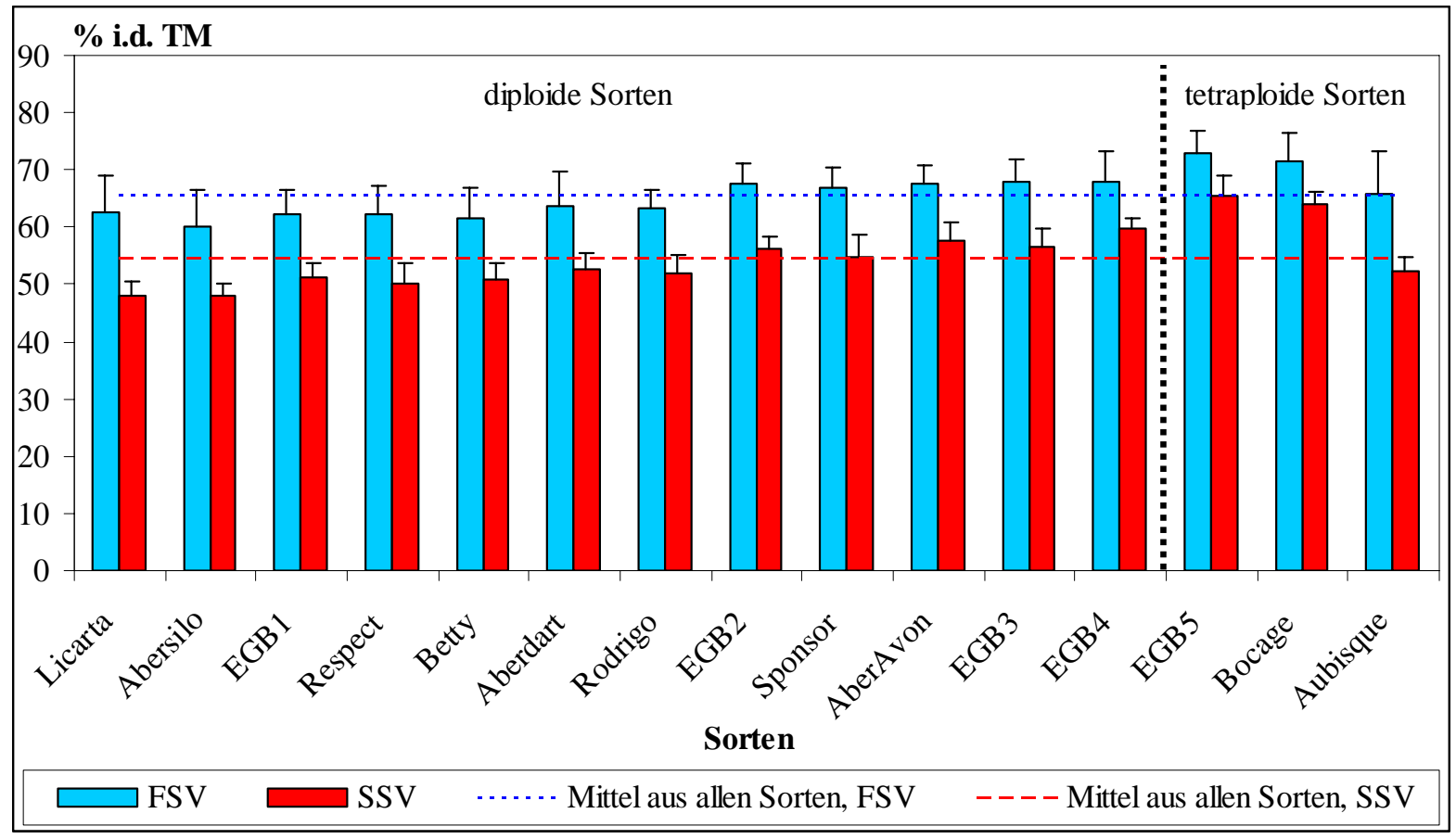

Abb. 23: Verdaulichkeit (\% i.d. TM) in tetraploiden und diploiden Sorten des Deutschen Weidelgrases, Mittelwert aus den beiden Versuchsstandorten BS und HS und den beiden Versuchsjahren 1HVJ und 2HVJ im 1. Schnitt bei frühem und spätem Schnittzeitpunkt.

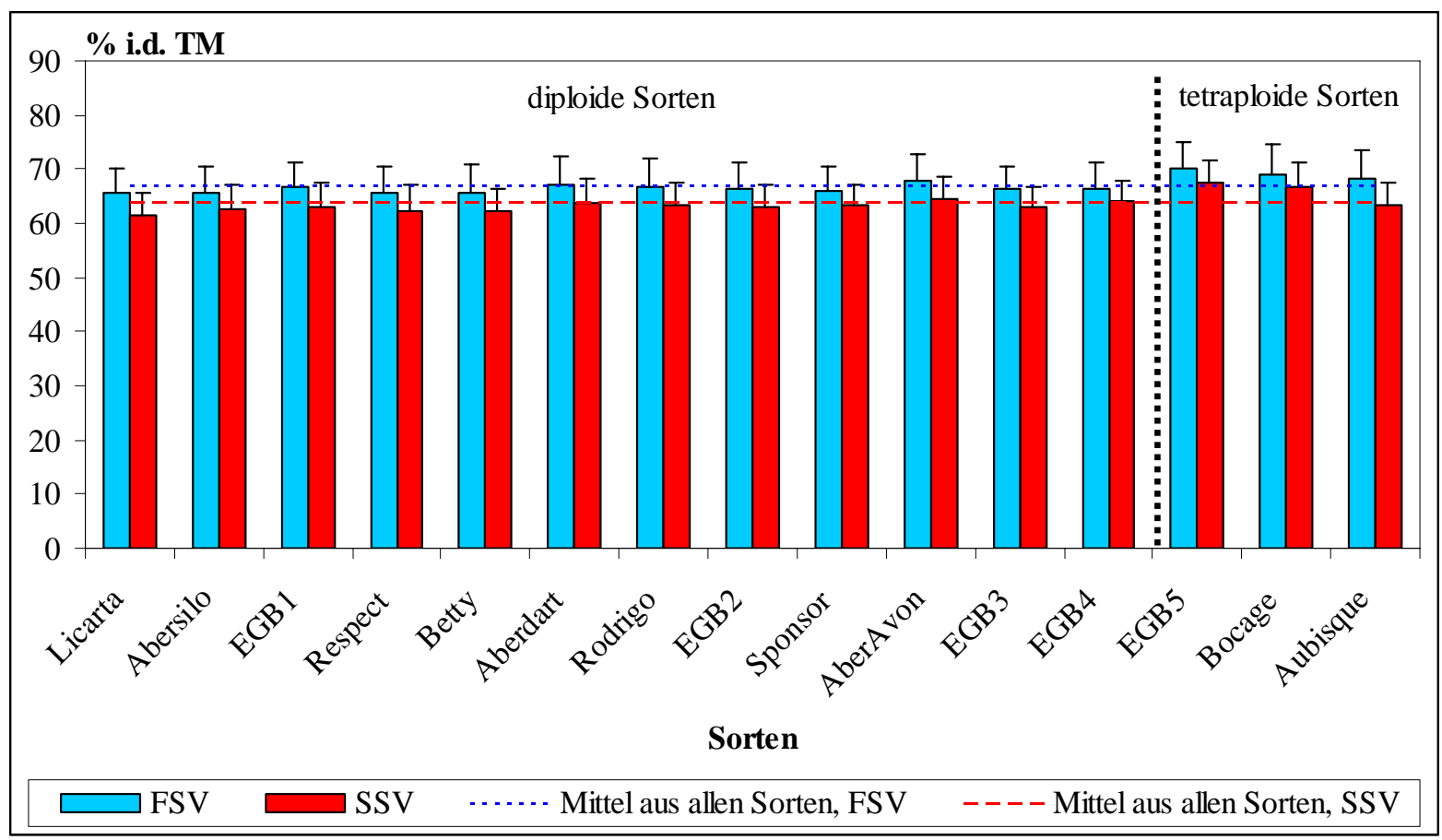

Abb. 24: Verdaulichkeit (\% i.d. TM) in tetraploiden und diploiden Sorten des Deutschen Weidelgrases, Mittelwert aus den beiden Versuchsstandorten BS und HS und den beiden Versuchsjahren $1 \mathrm{HVJ}$ und $2 \mathrm{HVJ}$ im Jahresmittel bei frühem und spätem Schnittzeitpunkt. 


\section{Die Verdaulichkeit im Verlauf der Vegetationsperiode}

Vom 1. zum 2. Schnitt stieg die Verdaulichkeit in der FSV im Sortenmittel an, vom 2. zum 3. Schnitt fiel sie dagegen ab, vom 3. zum 5. Schnitt stieg sie wieder an.

In der SSV stieg sie vom 1. zum 4. Schnitt stark an danach fiel die Verdaulichkeit von 4. zum 5. Schnitt ab (Abb. 25).

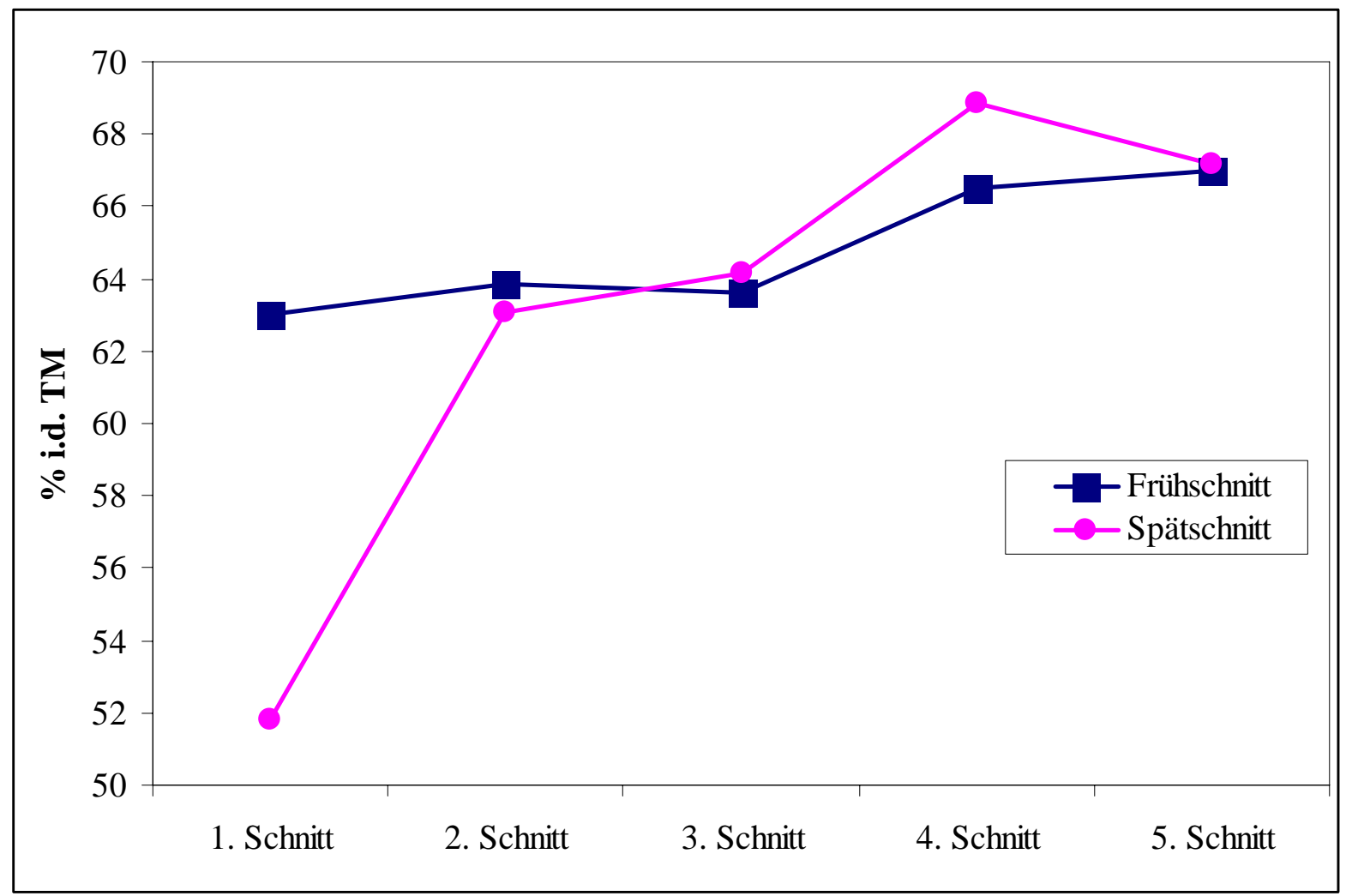

Abb. 25: Die Verdaulichkeit (\% i.d. TM) im Verlauf der Vegetationsperiode (Mittel aus allen 20 Sorten und den beiden Versuchsjahren 1HVJ und 2HVJ) an Versuchsstandort BS. 


\subsubsection{Rohproteingehalt}

Am Versuchsstandort BS lag der Rohproteingehalt (RP) im Mittel der Sorten im $\underline{1 \text {. Schnitt }}$ der FSV im 2HVJ (2007) mit 12,8 \% i.d. TM signifikant höher als im 1HVJ (2006) mit 11,8 \% i.d. TM. In der SSV wies der RP keine signifikanten Unterschiede zwischen 1HVJ und 2HVJ auf. Im Jahresmittel lag der RP im Mittel der Sorten in der FSV im 1HVJ mit 19,1 \% i.d. TM deutlich über dem Ergebnis des $2 \mathrm{HVJ}$ mit 17,3 \% i.d. TM und in der SSV im 1HVJ mit 20,7 \% i.d. TM auch über dem Ergebnis des 2HVJ mit 17,9 \% i.d. TM (Tab. 27).

Am Versuchsstandort HS lag der RP im Mittel der Sorten sowohl im $\underline{1 .}$ Schnitt als auch im Jahresmittel der FSV und der SSV im 1HVJ höher als im 2HVJ (Tab. 27).

Im Mittel der beiden Versuchsstandorten (BS und HS) im Mittel der Sorten im Jahresmittel der FSV lag der RP im 1HVJ mit 17,4 \% i.d. TM über dem Ergebnis des 2HVJ mit 16,1 \% i.d. TM. Auch lag der RP im Mittel der Sorten im Jahresmittel der SSV im 1HVJ mit 18,4 \% i.d. TM deutlich über dem Ergebnis des $2 \mathrm{HVJ}$ mit 16,7 \% i.d. TM.

Tab. 27: Versuchsjahresvergleich (2006/2007) für Rohproteingehalt (\% i.d. TM). Mittelwert aus allen Sorten im 1. Schnitt und im Jahresertrag.

\begin{tabular}{|c|c|c|c|c|c|c|c|c|c|c|c|c|}
\hline \multicolumn{13}{|l|}{ 1. Schnitt } \\
\hline \multirow{3}{*}{ Versuchsjahr } & \multicolumn{6}{|c|}{$\mathrm{BS}$} & \multicolumn{6}{|c|}{ HS } \\
\hline & \multicolumn{3}{|c|}{ FSV } & \multicolumn{3}{|c|}{ SSV } & \multicolumn{3}{|c|}{ FSV } & \multicolumn{3}{|c|}{ SSV } \\
\hline & Mw & & SD & $\mathrm{Mw}$ & & SD & Mw & & SD & Mw & & SD \\
\hline 2006 & 11,8 & $\mathrm{~b}$ & 1,2 & 9,0 & $\mathrm{a}$ & 1,1 & 16,3 & $\mathrm{a}$ & 1,7 & 13,7 & $\mathrm{a}$ & 2,0 \\
\hline 2007 & 12,8 & $\mathrm{a}$ & 1,6 & 8,9 & $\mathrm{a}$ & 1,0 & 11,2 & $\mathrm{~b}$ & 1,5 & 9,1 & $\mathrm{~b}$ & 1,3 \\
\hline \multicolumn{13}{|l|}{ Jahresmittel } \\
\hline 2006 & 19,1 & $\mathrm{a}$ & 0,8 & 20,7 & $\mathrm{a}$ & 1,0 & 15,7 & $\mathrm{a}$ & 1,3 & 16,1 & $\mathrm{a}$ & 1,0 \\
\hline 2007 & 17.3 & $\mathrm{~b}$ & 0,7 & 17,9 & $\mathrm{~b}$ & 0.7 & 14,9 & $\mathrm{~b}$ & 1.2 & 15,6 & $\mathrm{~b}$ & 0.7 \\
\hline
\end{tabular}

Signifikante Unterschiede gelten für Werte in derselben Spalten mit unterschiedlichen Buchstaben bei $\mathrm{P}<0,05$, $\mathrm{SD}=$ Standardabweichung, Mw = Mittelwert aus allen 20 Sorten und 3 Wiederholungen .

Sowohl am Versuchsstandort BS als auch am Versuchsstandort HS war der RP im Mittel der Sorten im $\underline{1}$. Schnitt des $1 \mathrm{HVJ}$ und des $2 \mathrm{HVJ}$ bei der FSV höher als bei der SSV. Dagegen war der RP im Jahresmittel im Mittel der Sorten im 1HVJ und im 2HVJ am Versuchsstandort BS und im 2HVJ am Versuchsstandort HS bei der SSV höher als bei der FSV. Im 1HVJ am Versuchsstandort HS wies der RP keine signifikanten Unterschiede zwischen den Schnittzeitpunktsvarianten auf (Tab. 28). 
Im Mittel der beiden Versuchjahre (1HVJ und 2HVJ) und der beiden Versuchsstandorten (BS und HS) war der RP im Mittel der Sorten im Jahresmittel bei der SSV (17,6 \% i.d. TM) höher als in der FSV (16,8 \% i.d. TM).

Tab. 28: Schnittzeitpunktvergleich (FSV/SSV) für Rohproteingehalt (\% i.d. TM). Mittelwert aus allen Sorten im 1. Schnitt und im Jahresmittel.

\begin{tabular}{|c|c|c|c|c|c|c|c|c|c|c|c|c|}
\hline \multicolumn{13}{|l|}{ 1. Schnitt } \\
\hline \multirow{3}{*}{ Schnittzeitpunkt } & \multicolumn{6}{|c|}{$\mathrm{BS}$} & \multicolumn{6}{|c|}{ HS } \\
\hline & \multicolumn{3}{|c|}{$1 \mathrm{HVJ}$} & \multicolumn{3}{|c|}{$2 \mathrm{HVJ}$} & \multicolumn{3}{|c|}{$1 \mathrm{HVJ}$} & \multicolumn{3}{|c|}{$2 \mathrm{HVJ}$} \\
\hline & Mw & & SD & Mw & & SD & Mw & & SD & Mw & & SD \\
\hline FSV & 11,8 & $\mathrm{a}$ & 1,2 & 12,7 & $\mathrm{a}$ & 1,6 & 16,3 & $\mathrm{a}$ & 1,7 & 11,2 & $\mathrm{a}$ & 1,5 \\
\hline SSV & 9,0 & $\mathrm{~b}$ & 1,1 & 9,0 & $\mathrm{~b}$ & 1,0 & 13,7 & $\mathrm{~b}$ & 2,0 & 9,1 & $\mathrm{~b}$ & 1,3 \\
\hline \multicolumn{13}{|l|}{ Jahresmittel } \\
\hline FSV & 19,1 & $\mathrm{~b}$ & 0,8 & 17,3 & $\mathrm{~b}$ & 0,7 & 15,7 & $\mathrm{a}$ & 1,3 & 14,9 & $\mathrm{~b}$ & 1,2 \\
\hline SSV & 20,7 & $\mathrm{a}$ & 1,0 & 17,9 & $\mathrm{a}$ & 0,7 & 16,1 & $\mathrm{a}$ & 1,0 & 15,6 & $\mathrm{a}$ & 0,7 \\
\hline
\end{tabular}

Signifikante Unterschiede gelten für Werte in derselben Spalte mit unterschiedlichen Buchstaben bei $\mathrm{P}<0,05$,

$\mathrm{SD}=$ Standardabweichung, $\mathrm{Mw}=$ Mittelwert aus allen 20 Sorten und 3 Wiederholungen .

Der RP war in der FSV und in der SSV des 1HVJ am Versuchsstandort HS höher als am Versuchsstandort BS. Dagegen war der RP in der FSV des 2HVJ am Versuchsstandort BS höher als am Versuchsstandort HS. In der SSV des 2HVJ wies der RP keine signifikanten Unterschiede zwischen den Versuchsstandorten auf (Tab. 29).

Im Mittel der beiden Versuchsjahren (1HVJ und 2HVJ) lag der RP im Mittel der Sorten im 1. Schnitt der FSV am HS mit 13,7 \% i.d. TM über dem Ergebnis am BS mit 12,3 \% i.d. TM.

Auch lag der RP im Mittel der Sorten im 1. Schnitt der FSV am HS mit 11,4 \% i.d. TM deutlich über dem Ergebnis am BS mit 9,0 \% i.d. TM.

Tab. 29: Rohproteingehalt (\% i.d. TM) an beiden Versuchsstandorte (BS und HS) im 1. Schnitt.

\begin{tabular}{|c|c|c|c|c|c|c|c|c|c|c|c|c|}
\hline \multirow{3}{*}{ Standort } & \multicolumn{6}{|c|}{$1 \mathrm{HVJ}$} & \multicolumn{6}{|c|}{$2 \mathrm{HVJ}$} \\
\hline & \multicolumn{3}{|c|}{ FSV } & \multicolumn{3}{|c|}{ SSV } & \multicolumn{3}{|c|}{ FSV } & \multicolumn{3}{|c|}{ SSV } \\
\hline & Mw & & SD & $\mathrm{Mw}$ & & SD & Mw & & SD & Mw & & SD \\
\hline $\mathrm{BS}$ & 11,8 & $\mathrm{~b}$ & 1,2 & 9,0 & $\mathrm{~b}$ & 1,1 & 12,7 & $\mathrm{a}$ & 1,6 & 9,0 & $\mathrm{a}$ & 1,0 \\
\hline HS & 16,3 & $\mathrm{a}$ & 1,7 & 13,7 & $\mathrm{a}$ & 2,0 & 11,2 & $\mathrm{~b}$ & 1,5 & 9,1 & $\mathrm{a}$ & 1,3 \\
\hline
\end{tabular}

Signifikante Unterschiede gelten für Werte in derselben Spalte mit unterschiedlichen Buchstaben bei $\mathrm{P}<0,05$, SD = Standardabweichung, $\mathrm{Mw}=$ Mittelwert aus allen 20 Sorten und 3 Wiederholungen .

\section{Grasarten- und Sortenunterschiede}

Bei der Frühschnittvariante wies Wiesenschwingelgras den höchsten RP (mit 17,2 \% i.d. TM) auf, Knaulgras enthielt dagegen die niedrigsten (mit 16,5 \% i.d. TM).

Bei der Spätschnittvariante wies Wiesenschweidelgras den höchsten RP (mit 17,8 \% i.d. TM) auf, Wiesenlieschgras enthielt dagegen die niedrigsten (mit 16,9 \% i.d. TM) (Abb. 26). 


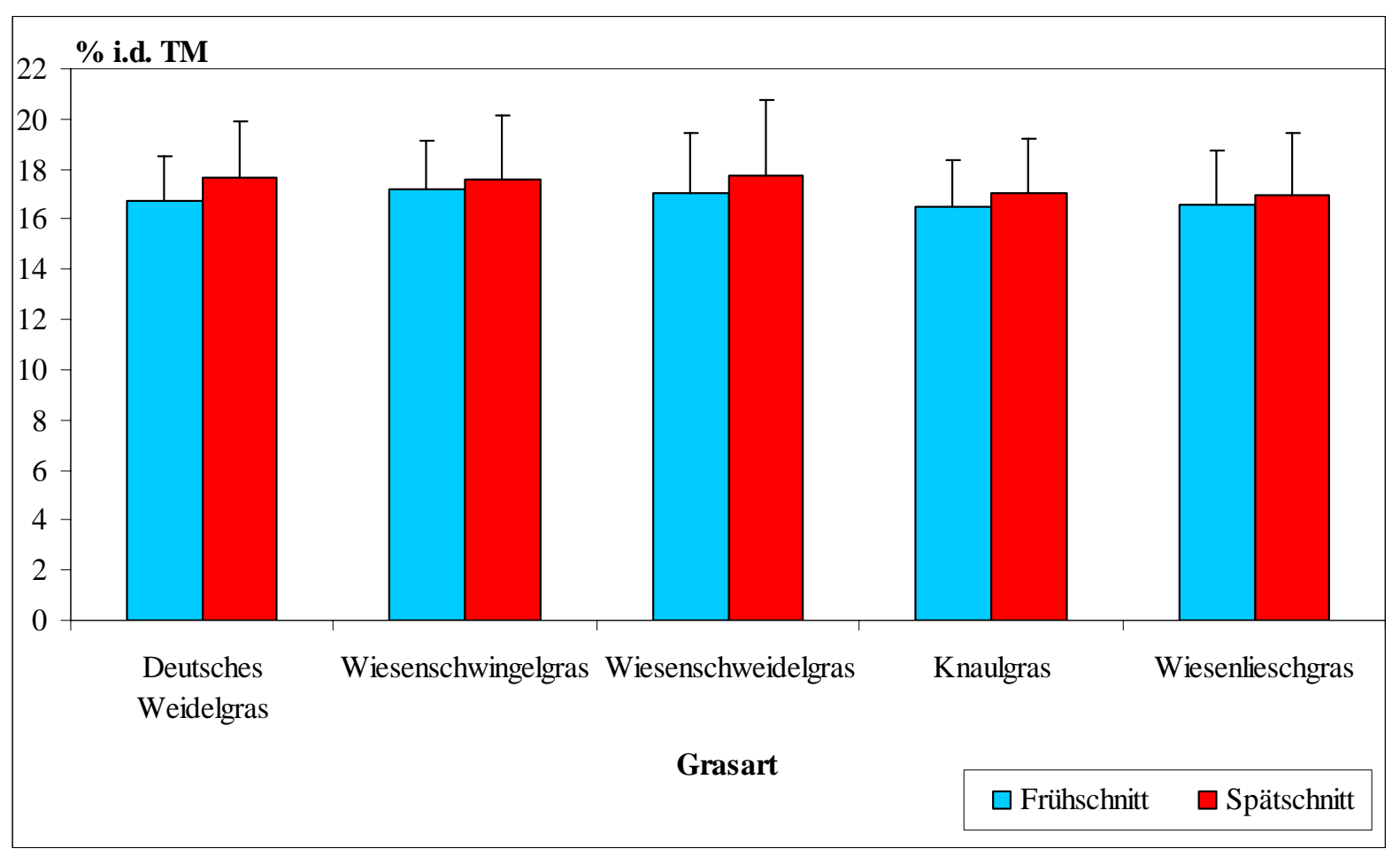

Abb. 26: Rohproteingehalt (\% i.d. TM) in verschiedenen Gräserarten des Grünlandes, Mittelwert aus den beiden Versuchsstandorten BS und HS und den beiden Versuchsjahren 1HVJ und 2HVJ bei frühem und spätem Schnittzeitpunkt.

Ein Blick auf die Abbildung 27 zeigt, dass der Rohproteingehalt in Deutschem Weidelgras im 1. Schnitt im Mittel der beiden Versuchsjahre (1HVJ und 2HVJ) und der beiden Versuchsstandorten (BS und HS) deutlich von späten zu frühen Sorten abnahm (Abb. 27).

In derselben Reifegruppen im 1. Schnitt der FSV wiesen die Sorten Licarta (12,9 \% i.d. TM) für früh, EGB2 (13,6 i.d. TM) für mittel und EGB4 (14,5 \% i.d. TM) für spät den höchsten Rohproteingehalt auf. Im 1. Schnitt der SSV wiesen die Sorten die Sorten EGB1 (9,8 \% i.d. TM) für früh, EGB2 (10,9 i.d. TM) für mittel und EGB3 (11,9 \% i.d. TM) für spät den höchsten Rohproteingehalt auf (Abb. 27). 


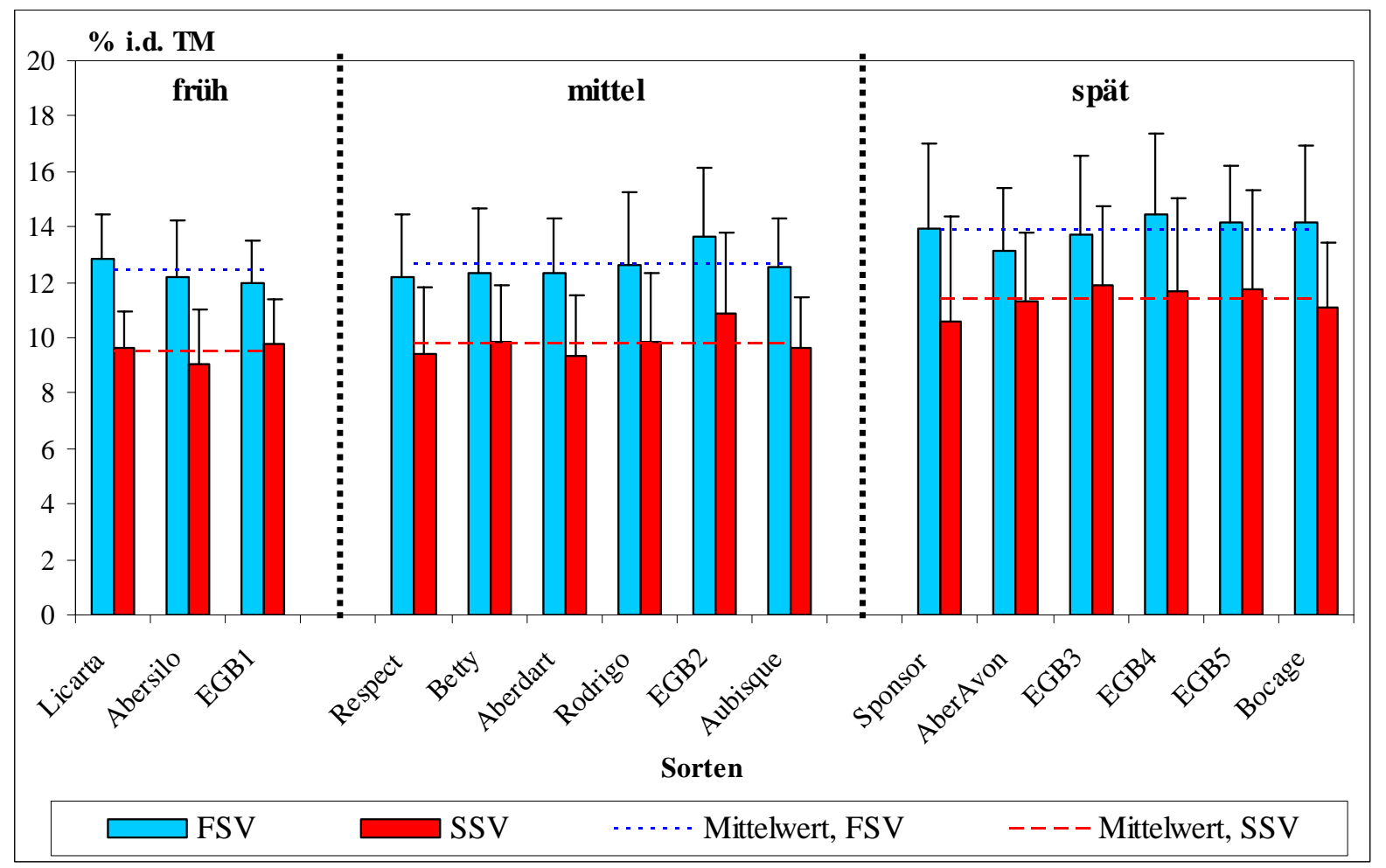

Abb. 27: Rohproteingehalt (\% i.d. TM) von Deutschen Weidelgrassorten der Reifegruppe früh, mittel und spät, Mittelwert aus den beiden Versuchsstandorten BS und HS und den beiden Versuchsjahren 1HVJ und 2HVJ im 1. Schnitt bei frühem und spätem Schnittzeitpunkt.

Der Rohproteingehalt war bei den tetraploiden Sorten des Deutschen Weidelgrases höher als bei den diploiden Sorten (Abb. 28).

Im Mittel der beiden Versuchsjahre (1HVJ und 2HVJ) und der beiden Versuchsstandorten (BS und HS) wiesen die Sorte EGB4 (14,5 \% i.d. TM) in der Diploidegruppe und die Sorten EGB5 und Bocage (14,1 \% i.d. TM) in der Tetraploidegruppe im 1. Schnitt der FSV die höchsten Rohproteingehalt auf. Im 1. Schnitt der SSV wiesen die Sorten EGB3 (11,9 \% i.d. TM) in der Diploidegruppe und die Sorte EGB5 (11,8 \% i.d. TM) in der Tetraploidegruppe den höchsten Rohproteingehalt auf (Abb. 28).

Im Jahresmittel der FSV wiesen die Sorten Licarta und Sponsor (17,3 \% i.d. TM) in der Diploidegruppe und die Sorte Bocage (17,4 \% i.d. TM) in der Tetraploidegruppe den höchsten Rohproteingehalt auf. Im Jahresmittel der SSV wiesen die Sorten Sponsor (18,2 \% i.d. TM) in der Diploidegruppe und die Sorte EGB5 (18 \% i.d. TM) in der Tetraploidegruppe den höchsten Rohproteingehalt auf (Abb. 29). 


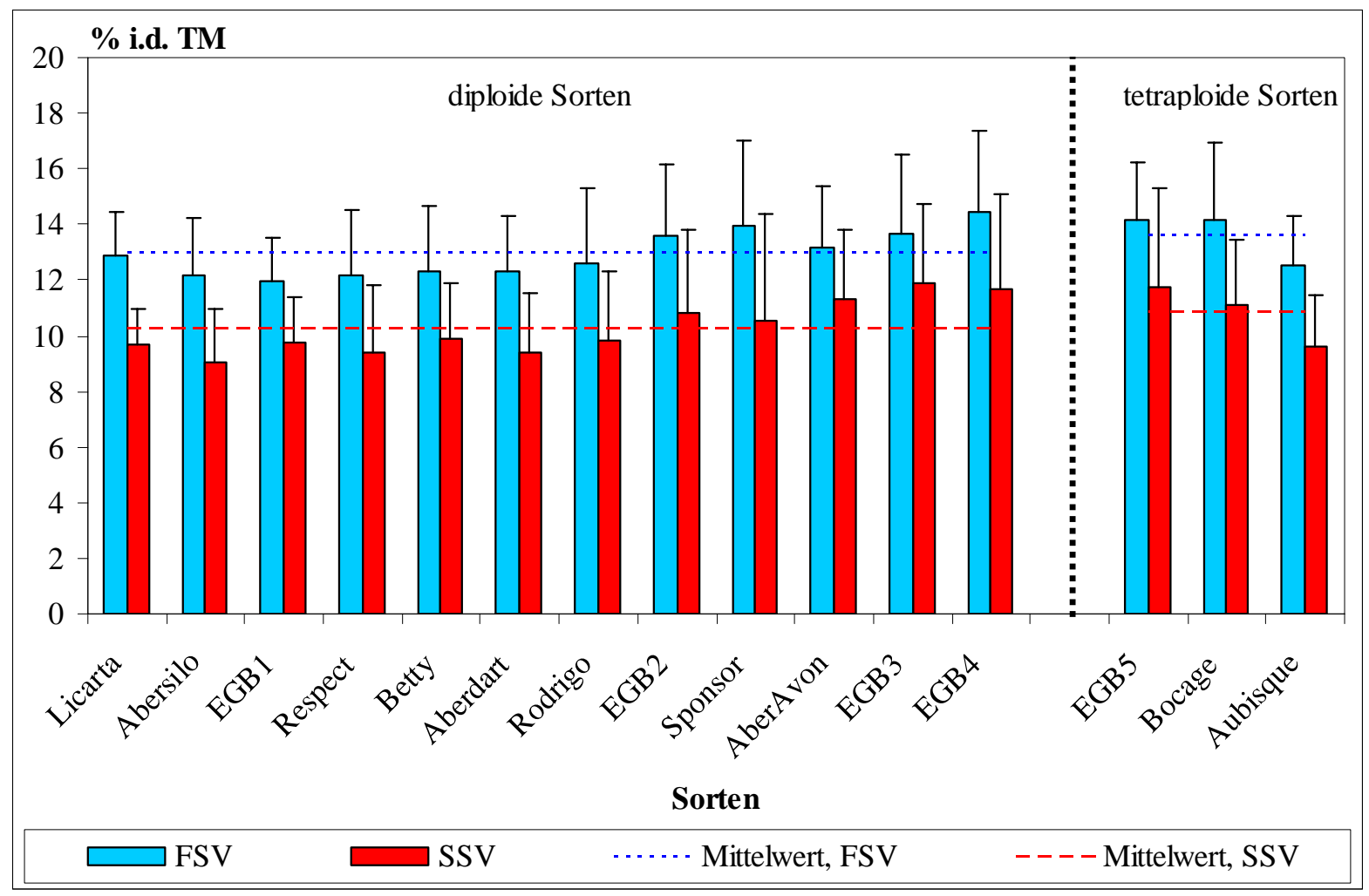

Abb. 28: Rohproteingehalt (\% i.d. TM) in tetraploiden und diploiden Sorten des Deutschen Weidelgrases, Mittelwert aus den beiden Versuchsstandorten BS und HS und den beiden Versuchsjahren 1HVJ und 2HVJ im 1. Schnitt bei frühem und spätem Schnittzeitpunkt.

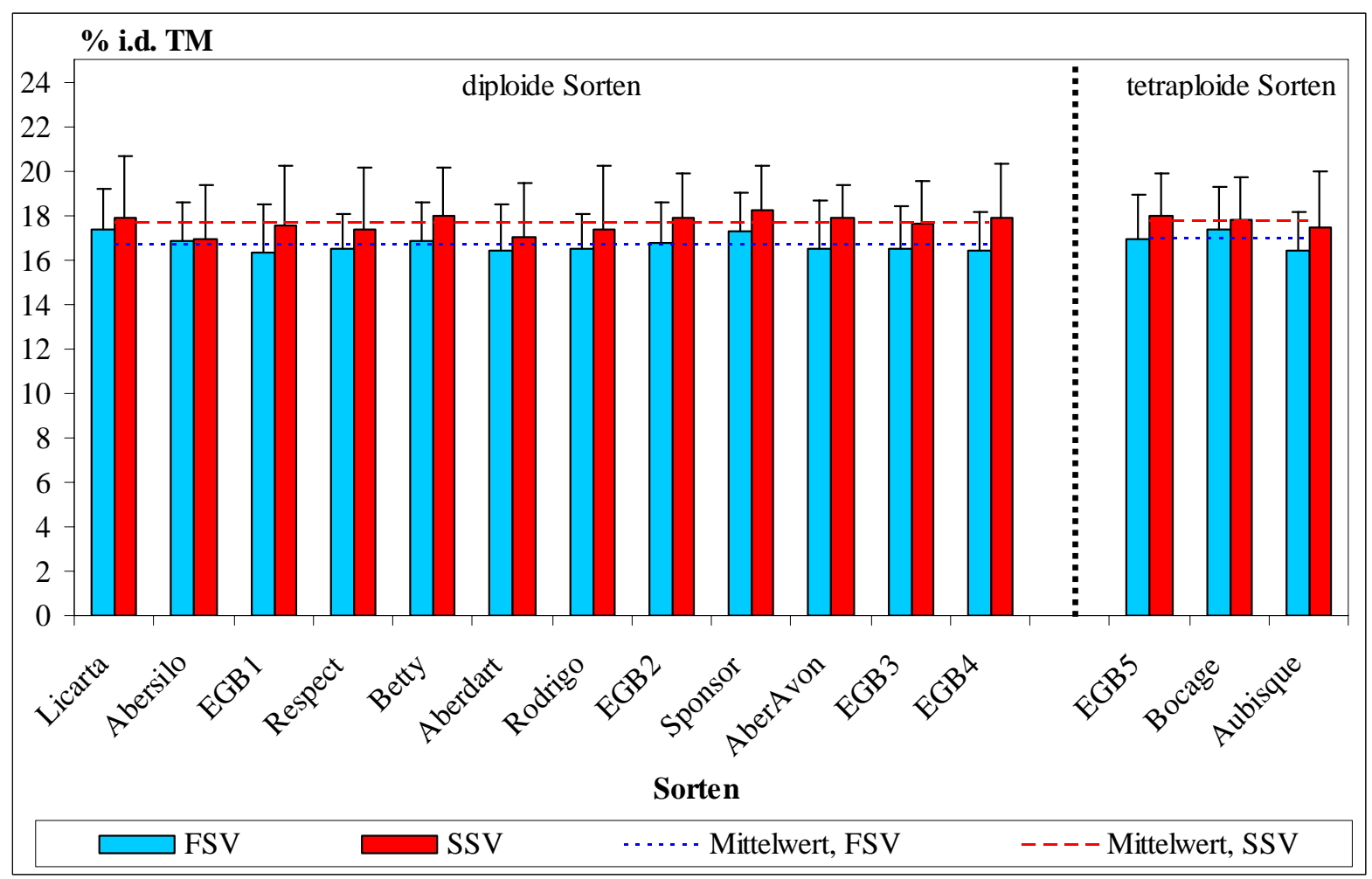

Abb. 29: Rohproteingehalt (\% i.d. TM) in tetraploiden und diploiden Sorten des Deutschen Weidelgrases, Mittelwert aus den beiden Versuchsstandorten BS und HS und den beiden Versuchsjahren 1HVJ und 2HVJ im Jahresmittel bei frühem und spätem Schnittzeitpunkt. 


\section{Der Rohproteingehalt im Verlauf der Vegetationsperiode}

Vom 1. zum 5. Schnitt stieg der RP in der FSV im Sortenmittel an.

In der SSV stieg der RP vom 1. zum 3. Schnitt stark an, vom 3. zum 4. Schnitt fiel dagegen ab, vom 4. zum 5. Schnitt stieg wieder an (Abb. 30).

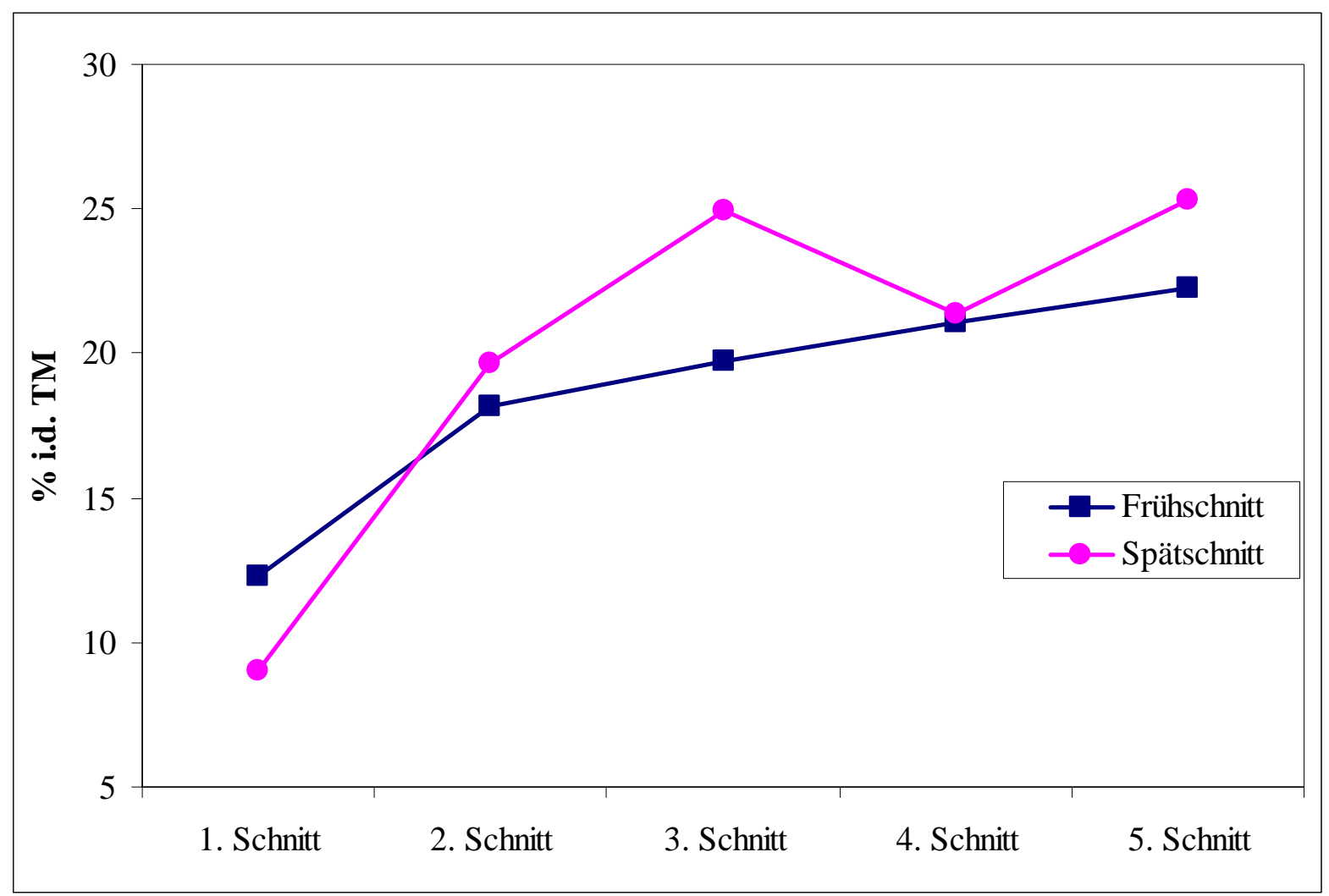

Abb. 30: Rohproteingehalt (\% i.d. TM) im Verlauf der Vegetationsperiode (Mittel aus allen 20 Sorten und den beiden Versuchsjahren 1HVJ und 2HVJ) an Versuchsstandort BS. 


\subsubsection{Rohfasergehalt}

Am Versuchsstandort BS lag der Rohfasergehalt (RF) im Mittel der Sorten im $\underline{1 .}$ Schnitt der FSV im 1HVJ (2006) mit 27,1 \% i.d. TM signifikant höher als im 2HVJ (2007) mit 22,1 \% i.d. TM. Dagegen lag der RF in der SSV 2HVJ mit 30,1 \% i.d. TM signifikant höher als im 1HVJ mit 27,5 \% i.d. TM.

Im Jahresmittel lag der RF im Mittel der Sorten in der FSV im 1HVJ mit 24,5 \% i.d. TM über dem Ergebnis des 2HVJ mit 23,0 \% i.d. TM. In der SSV wies der RF keine signifikanten Unterschiede zwischen 1HVJ und 2HVJ auf.

Am Versuchsstandort HS wies der RF im Mittel der Sorten im $\underline{1 .}$ Schnitt der FSV und der SSV keine signifikanten Unterschiede zwischen 1HVJ und 2HVJ auf.

Im Jahresmittel lag der RF im Mittel der Sorten sowohl in der FSV als auch in der SSV im 1HVJ höher als im 2HVJ (Tab. 30).

Im Mittel der beiden Versuchsstandorten (BS und HS) im Mittel der Sorten im Jahresmittel der FSV lag der RF im 1HVJ mit 25,0 \% i.d. TM über dem Ergebnis des 2HVJ mit 23,9 \% i.d. TM. Auch lag der RF im Mittel der Sorten im Jahresmittel der SSV im 1HVJ mit 26,0 \% i.d. TM über dem Ergebnis des 2HVJ mit 25,5 \% i.d. TM.

Tab. 30: Versuchsjahresvergleich (2006/2007) für Rohfasergehalt (\% i.d. TM). Mittelwert aus allen Sorten im 1. Schnitt und im Jahresertrag.

\begin{tabular}{|c|c|c|c|c|c|c|c|c|c|c|c|c|}
\hline \multicolumn{13}{|l|}{ 1. Schnitt } \\
\hline \multirow{3}{*}{ Versuchsjahr } & \multicolumn{6}{|c|}{ BS } & \multicolumn{6}{|c|}{$\mathrm{HS}$} \\
\hline & \multicolumn{3}{|c|}{ FSV } & \multicolumn{3}{|c|}{ SSV } & \multicolumn{3}{|c|}{ FSV } & \multicolumn{3}{|c|}{ SSV } \\
\hline & Mw & & $\mathrm{SD}$ & $\mathrm{Mw}$ & & SD & $\mathrm{Mw}$ & & SD & Mw & & SD \\
\hline 2006 & 27,1 & $\mathrm{a}$ & 4,6 & 27,5 & $\mathrm{~b}$ & 4,3 & 27,3 & $\mathrm{a}$ & 2,7 & 31,1 & $\mathrm{a}$ & 3,2 \\
\hline 2007 & 22,1 & $\mathrm{~b}$ & 3,3 & 30,1 & $\mathrm{a}$ & 3,5 & 27,0 & $\mathrm{a}$ & 2,7 & 31,5 & $\mathrm{a}$ & 2,6 \\
\hline \multicolumn{13}{|l|}{ Jahresmittel } \\
\hline 2006 & 24,5 & $\mathrm{a}$ & 1,8 & 25,0 & $\mathrm{a}$ & 1,6 & 25,6 & $\mathrm{a}$ & 1,4 & 27,0 & $\mathrm{a}$ & 1,5 \\
\hline 2007 & 23,0 & $\mathrm{~b}$ & 1,9 & 25,1 & $\mathrm{a}$ & 1,8 & 24,8 & $\mathrm{~b}$ & 1,8 & 26,0 & $\mathrm{~b}$ & 1,9 \\
\hline
\end{tabular}

Signifikante Unterschiede gelten für Werte in derselben Spalten mit unterschiedlichen Buchstaben bei $\mathrm{P}<0,05$, $\mathrm{SD}=$ Standardabweichung, $\mathrm{Mw}=$ Mittelwert aus allen 20 Sorten und 3 Wiederholungen .

Sowohl am Versuchsstandort BS als auch am Versuchsstandort HS war der RF im Mittel der Sorten in beiden HVJ im 1. Schnitt und im Jahresmittel bei der FSV höher als bei der SSV. (Tab. 31).

Im Mittel der beiden Versuchjahre (1HVJ und 2HVJ) und der beiden Versuchsstandorten (BS und HS) war der RF im Mittel der Sorten im Jahresmittel bei der SSV (25,7 \% i.d. TM) höher als in der FSV (24,5 \% i.d. TM). 
In der FSV des $1 \mathrm{HVJ}$ wies der RF keine signifikanten Unterschiede zwischen den Versuchsstandorten auf. Der RF war in der SSV des 1HVJ und in der FSV und in der SSV des 2HVJ am Versuchsstandort HS höher als am Versuchsstandort BS (Tab. 32).

Im Mittel der beiden Versuchsjahren (1HVJ und 2HVJ) lag der RF im Mittel der Sorten im 1. Schnitt der FSV am HS mit 27,2 \% i.d. TM über dem Ergebnis am BS mit 24,6 \% i.d. TM.

Auch lag der RF im Mittel der Sorten im 1. Schnitt der FSV am HS mit 31,3 \% i.d. TM deutlich über dem Ergebnis am BS mit 28,8 \% i.d. TM.

Tab. 31: Schnittzeitpunktvergleich (FSV/SSV) für Rohfasergehalt (\% i.d. TM). Mittelwert aus allen Sorten im 1. Schnitt und im Jahresmittel.

\begin{tabular}{|c|c|c|c|c|c|c|c|c|c|c|c|c|}
\hline \multicolumn{13}{|l|}{ 1. Schnitt } \\
\hline \multirow{3}{*}{ Schnittzeitpunkt } & \multicolumn{6}{|c|}{ BS } & \multicolumn{6}{|c|}{ HS } \\
\hline & \multicolumn{3}{|c|}{$1 \mathrm{HVJ}$} & \multicolumn{3}{|c|}{$2 \mathrm{HVJ}$} & \multicolumn{3}{|c|}{$1 \mathrm{HVJ}$} & \multicolumn{3}{|c|}{$2 \mathrm{HVJ}$} \\
\hline & Mw & & SD & Mw & & SD & Mw & & SD & $\mathrm{Mw}$ & & $\overline{\mathrm{SD}}$ \\
\hline FSV & 27,1 & $\mathrm{~b}$ & 4,6 & 22,1 & $\mathrm{~b}$ & 3,3 & 27,3 & $\mathrm{~b}$ & 2,7 & 27,0 & $\mathrm{~b}$ & 2,7 \\
\hline SSV & 27,5 & $\mathrm{a}$ & 4,4 & 30,1 & $\mathrm{a}$ & 3,5 & 31,1 & $\mathrm{a}$ & 3,2 & 31,5 & $\mathrm{a}$ & 2,6 \\
\hline \multicolumn{13}{|l|}{ Jahresmittel } \\
\hline FSV & 24,5 & $\mathrm{~b}$ & 1,8 & 23,0 & $\mathrm{~b}$ & 1,9 & 25,6 & $\mathrm{~b}$ & 1,4 & 24,8 & $\mathrm{~b}$ & 1,8 \\
\hline SSV & 25,0 & $\mathrm{a}$ & 1,6 & 25,1 & $\mathrm{a}$ & 1,8 & 26,9 & $\mathrm{a}$ & 1,5 & 25,9 & $\mathrm{a}$ & 1,7 \\
\hline
\end{tabular}

Signifikante Unterschiede gelten für Werte in derselben Spalte mit unterschiedlichen Buchstaben bei $\mathrm{P}<0,05$, $\mathrm{SD}=$ Standardabweichung, $\mathrm{Mw}=$ Mittelwert aus allen 20 Sorten und 3 Wiederholungen .

Tab. 32: Rohfasergehalt (\% i.d. TM) an beiden Versuchsstandorte (BS und HS) im 1. Schnitt.

\begin{tabular}{|c|c|c|c|c|c|c|c|c|c|c|c|c|}
\hline \multirow{3}{*}{ Standort } & \multicolumn{6}{|c|}{$1 \mathrm{HVJ}$} & \multicolumn{6}{|c|}{$2 \mathrm{HVJ}$} \\
\hline & \multicolumn{3}{|c|}{ FSV } & \multicolumn{3}{|c|}{ SSV } & \multicolumn{3}{|c|}{ FSV } & \multicolumn{3}{|c|}{ SSV } \\
\hline & Mw & & SD & Mw & & SD & Mw & & SD & Mw & & SD \\
\hline $\mathrm{BS}$ & 27,0 & $\mathrm{a}$ & 4,6 & 27,5 & $\mathrm{~b}$ & 4,3 & 22,1 & $\mathrm{~b}$ & 3,3 & 30,1 & $\mathrm{~b}$ & 3,5 \\
\hline $\mathrm{HS}$ & 27,3 & $\mathrm{a}$ & 2,7 & 31,1 & $\mathrm{a}$ & 3,2 & 27,0 & $\mathrm{a}$ & 2,7 & 31,5 & $\mathrm{a}$ & 2,6 \\
\hline
\end{tabular}

Signifikante Unterschiede gelten für Werte in derselben Spalte mit unterschiedlichen Buchstaben bei $\mathrm{P}<0,05$, $\mathrm{SD}=$ Standardabweichung, $\mathrm{Mw}=$ Mittelwert aus allen 20 Sorten und 3 Wiederholungen.

\section{Grasarten- und Sortenunterschiede}

Sowohl bei der Frühschnittvariante als auch bei der Spätschnittvariante wies Knaulgras den höchsten RF auf, Deutsches Weidelgras enthielt dagegen die niedrigsten (Abb. 31). 


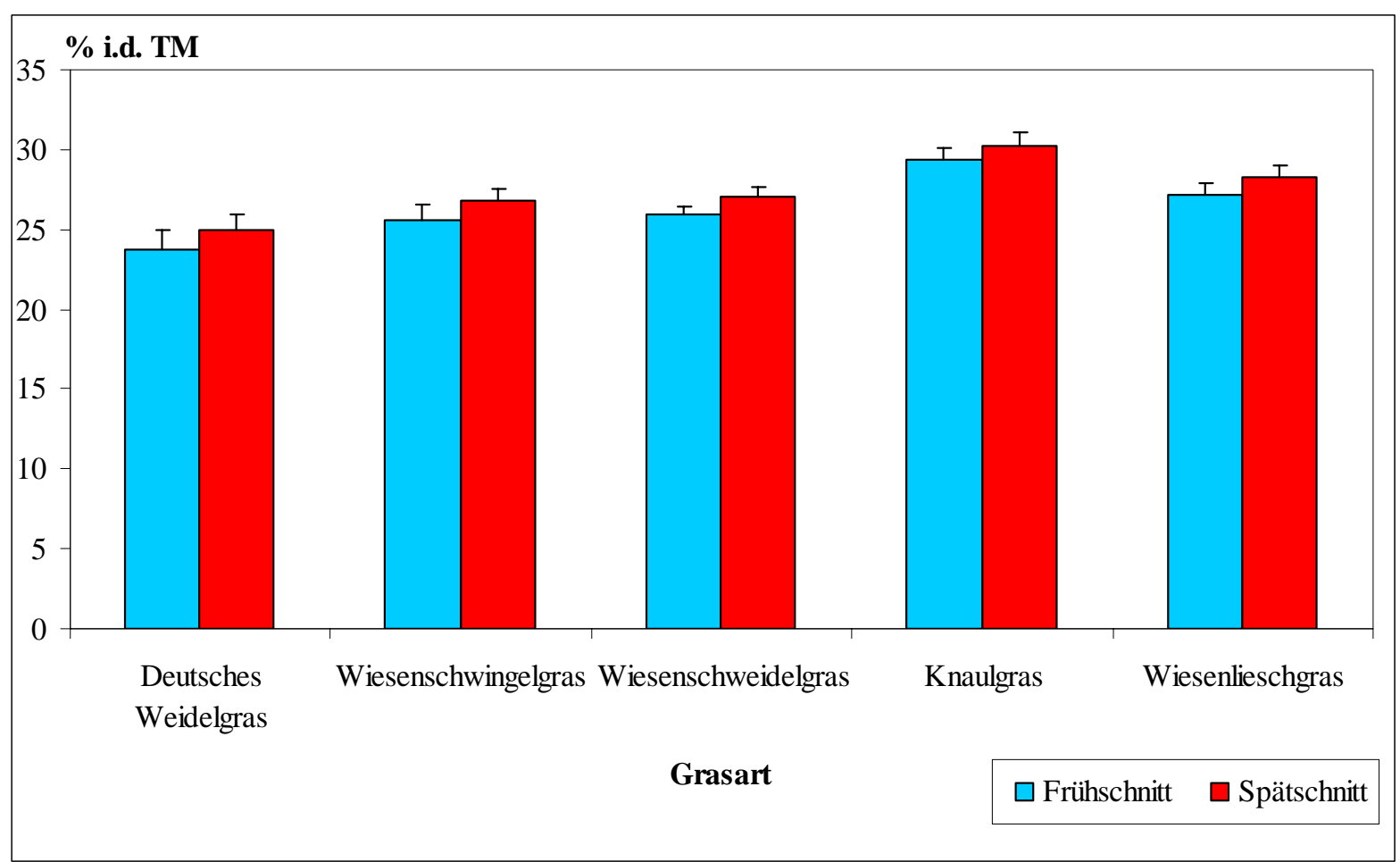

Abb. 31: Rohfasergehalt (\% i.d. TM) in verschiedenen Gräserarten des Grünlandes, Mittelwert aus den beiden Versuchsstandorten BS und HS und den beiden Versuchsjahren 1HVJ und $2 \mathrm{HVJ}$ bei frühem und spätem Schnittzeitpunkt.

Der Rohfasergehalt in Deutschem Weidelgras im 1. Schnitt im Mittel der beiden Versuchsjahre (1HVJ und 2HVJ) und der beiden Versuchsstandorten (BS und HS) nahm deutlich von frühen zu späten Sorten ab (Abb. 32).

In derselben Reifegruppen im 1. Schnitt der FSV wiesen die Sorten Abersilo (27,2 \% i.d. TM) für früh, Respect (26,5 \% i.d. TM) für mittel und Sponsor (24,3 \% i.d. TM) für spät die höchsten Rohfasergehalt auf. Im 1. Schnitt der SSV wiesen die Sorten die Sorten Licarta und Abersilo (31,3 \% i.d. TM) für früh, Respect (30,9 \% i.d. TM) für mittel und Sponsor (28,6 \% i.d. TM) für spät die höchsten Rohproteingehalt auf (Abb. 32). 


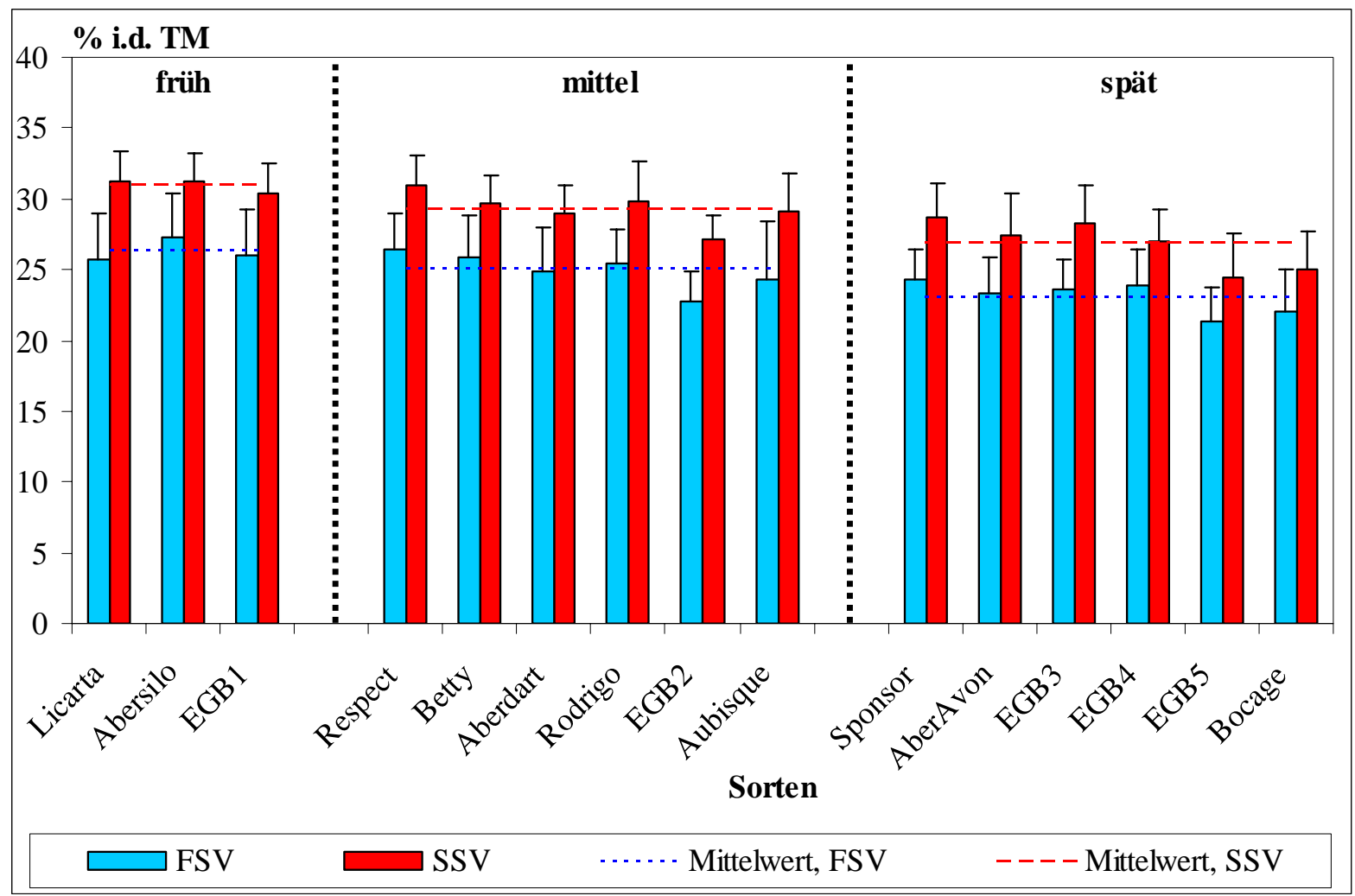

Abb. 32: Rohfasergehalt (\% i.d. TM) von Deutschen Weidelgrassorten der Reifegruppe früh, mittel und spät, Mittelwert aus den beiden Versuchsstandorten BS und HS und den beiden Versuchsjahren 1HVJ und 2HVJ im 1. Schnitt bei frühem und spätem Schnittzeitpunkt.

Der Rohfasergehalt war bei den diploiden Sorten des Deutschen Weidelgrases höher als bei den tetraploiden Sorten (Abb. 33).

Im Mittel der beiden Versuchsjahre (1HVJ und 2HVJ) und der beiden Versuchsstandorten (BS und HS) wiesen die Sorten Abersilo (27,2 \% i.d. TM) in der Diploidegruppe und die Sorte Aubisque (24,3 \% i.d. TM) in der Tetraploidegruppe im 1. Schnitt der FSV die höchsten Rohfasergehalt auf. Im 1. Schnitt der SSV wiesen die Sorten Licarta und Abersilo (31,3 \% i.d. TM) in der Diploidegruppe und die Sorte Aubisque (29,1 \% i.d. TM) in der Tetraploidegruppe den höchsten Rohfasergehalt auf (Abb. 33).

Im Jahresmittel der FSV wiesen die Sorte Respect (24,9 \% i.d. TM) in der Diploidegruppe und die Sorte Aubisque (23,2 \% i.d. TM) in der Tetraploidegruppe den höchsten Rohfasergehalt auf. Im Jahresmittel der SSV wiesen die Sorte Respect (26,2 \% i.d. TM) in der Diploidegruppe und die Sorte Aubisque (24,8 \% i.d. TM) in der Tetraploidegruppe den höchsten Rohfasergehalt auf (Abb. 34). 


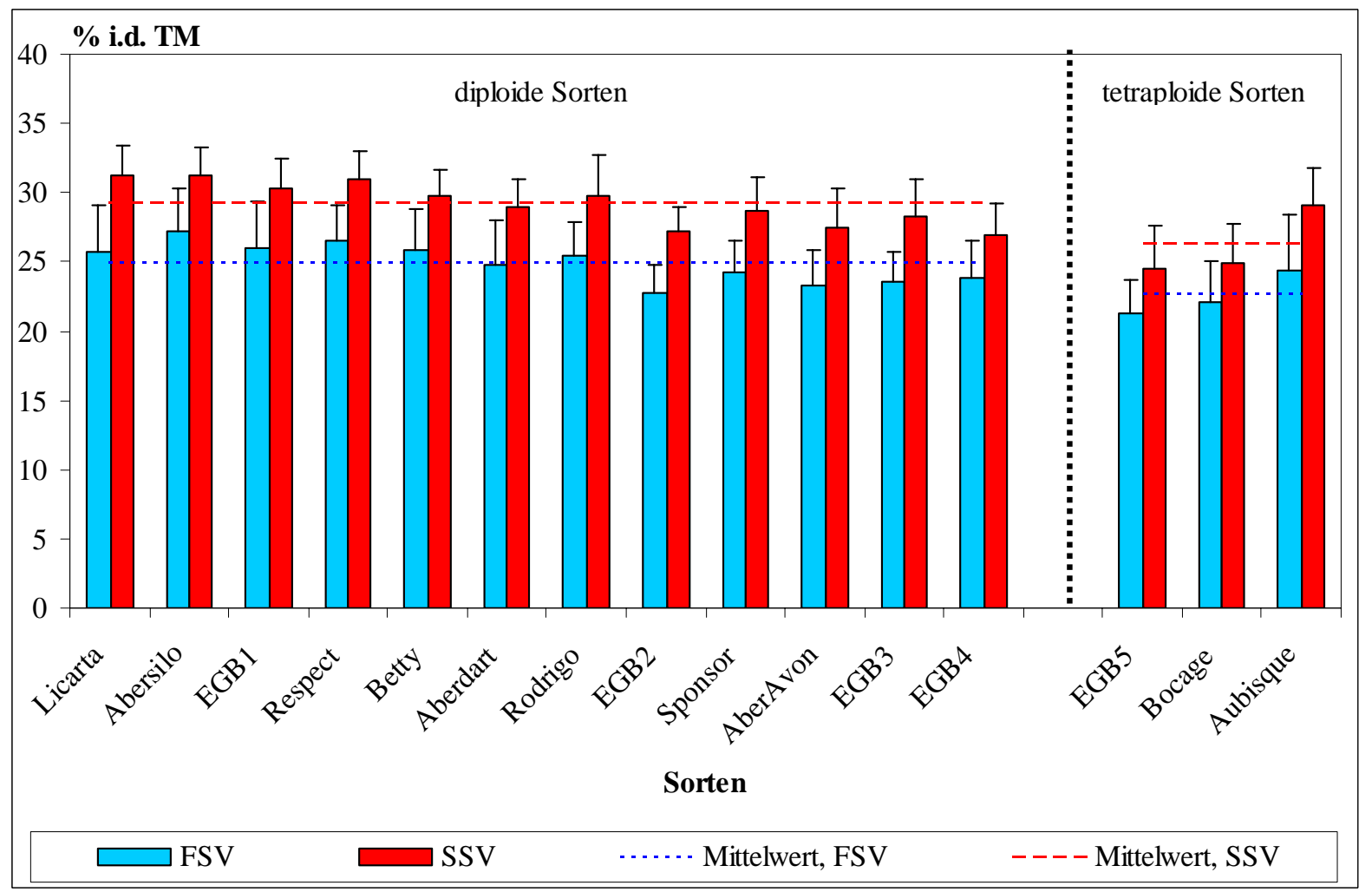

Abb. 33: Rohfasergehalt (\% i.d. TM) in tetraploiden und diploiden Sorten des Deutschen Weidelgrases, Mittelwert aus den beiden Versuchsstandorten BS und HS und den beiden Versuchsjahren 1HVJ und 2HVJ im 1. Schnitt bei frühem und spätem Schnittzeitpunkt.

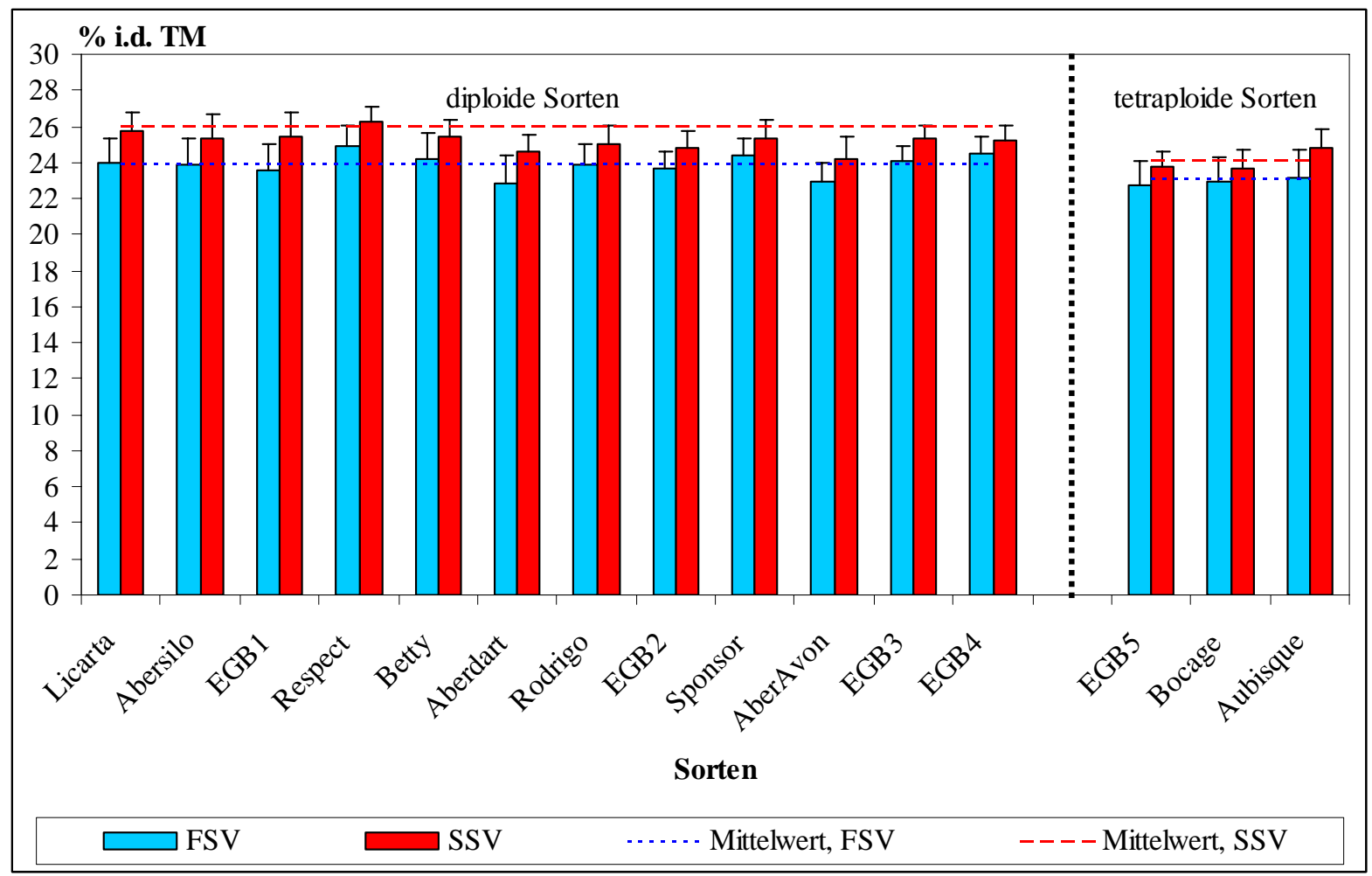

Abb. 34: Rohfasergehalt (\% i.d. TM) in tetraploiden und diploiden Sorten des Deutschen Weidelgrases, Mittelwert aus den beiden Versuchsstandorten BS und HS und den beiden Versuchsjahren 1HVJ und 2HVJ im Jahresmittel bei frühem und spätem Schnittzeitpunkt. 


\section{Der Rohfasergehalt im Verlauf der Vegetationsperiode}

Vom 1. zum 2. Schnitt fiel der RF in der Frühschnittsvariante (FSV) im Sortenmittel ab, vom

2. zum 3. Schnitt stieg leicht an, vom 3. zum 4. Schnitt fiel wieder leicht ab, vom 4. zum 5. Schnitt stieg wieder leicht an.

In der Spätschnittsvariante (SSV) fiel der RF vom 1. zum 2. Schnitt stark ab, vom 2. zum 4. Schnitt stieg dagegen an, vom 4. zum 5. Schnitt fiel er wieder ab (Abb. 35).

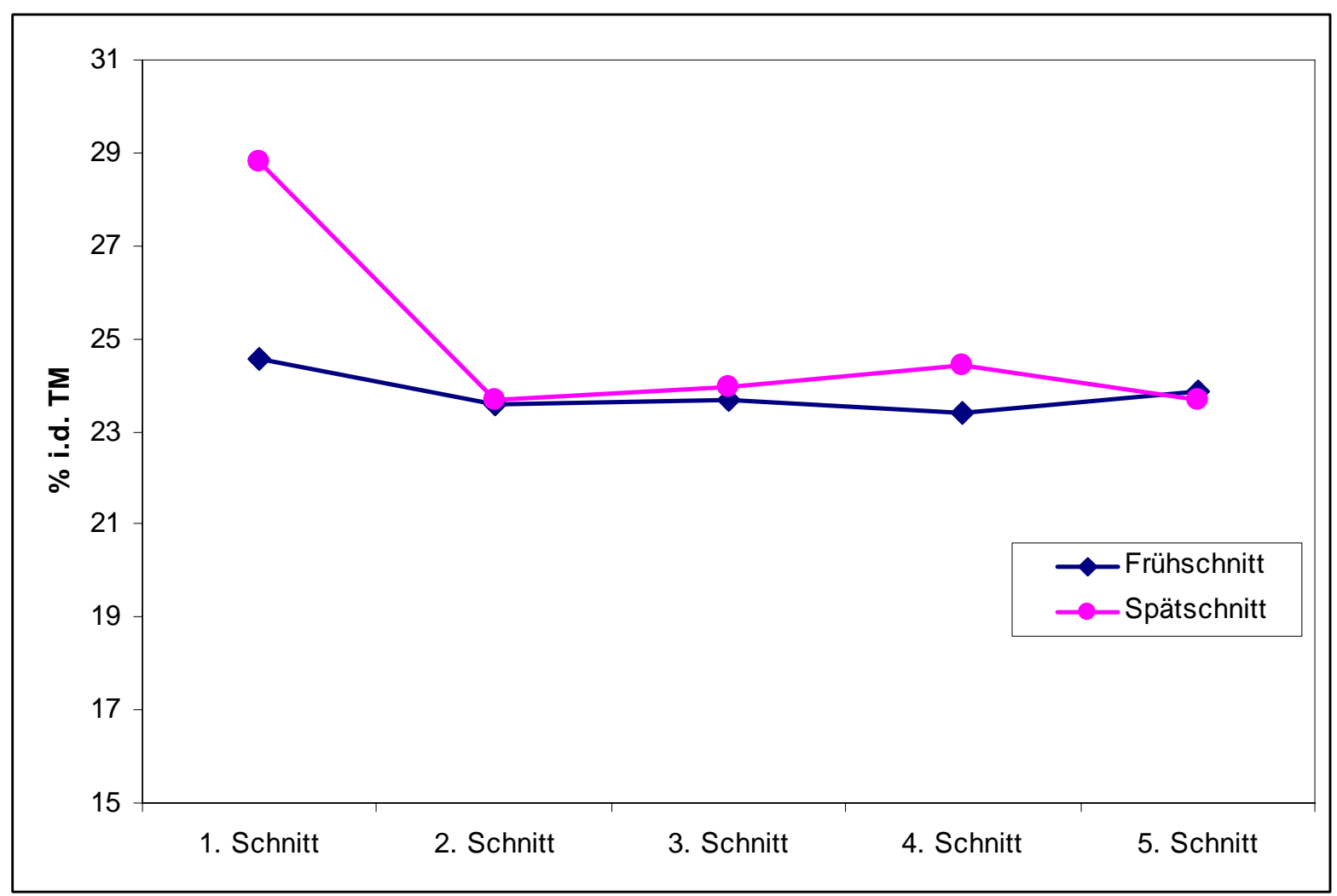

Abb. 35: Rohfasergehalt (\% i.d. TM) im Verlauf der Vegetationsperiode (Mittel aus allen 20 Sorten und den beiden Versuchsjahren $1 \mathrm{HVJ}$ und 2HVJ) an Versuchsstandort BS. 


\subsubsection{Die Korrelationen zwischen den wasserlöslichen Kohlenhydraten und dem TM- Ertrag, der Verdaulichkeit, dem Rohproteingehalt und dem Rohfasergehalt.}

Im Sortenmittel, im Jahresmittel und im Mittel der beiden Versuchsjahre (1HVJ und 2HVJ), der beiden Versuchsstandorten (BS und HS) und der Schnittzeitpunktsvarianten (FSV und SSV) war der Zusammenhang zwischen dem wasserlöslichen Kohlenhydrate-Gehalt und dem TM-Ertrag signifikant. Die beiden Parameter korrelieren negativ miteinander wie die Abbildung 36 verdeutlicht.

Der wasserlöslicher Kohlenhydrate-Gehalt und die Verdaulichkeit befinden sich in einem hoch signifikanten Zusammenhang, wobei steigende Verdaulichkeit im Gras von steigendem wasserlöslichem Kohlenhydrate-Gehalt begleitet wird (Abb. 37).

Wurden der Rohprotein- und Rohfasergehalt im Gras in Beziehungen gesetzt zum wasserlöslichen Kohlenhydrate-Gehalt, so ergibt sich ebenfalls ein statistisch abgesicherter Zusammenhang. Der Rohprotein- und Rohfasergehalt korrelieren negativ mit wasserlöslichem Kohlenhydrate-Gehalt wie die Abbildungen 38 und 39 verdeutlichen. 


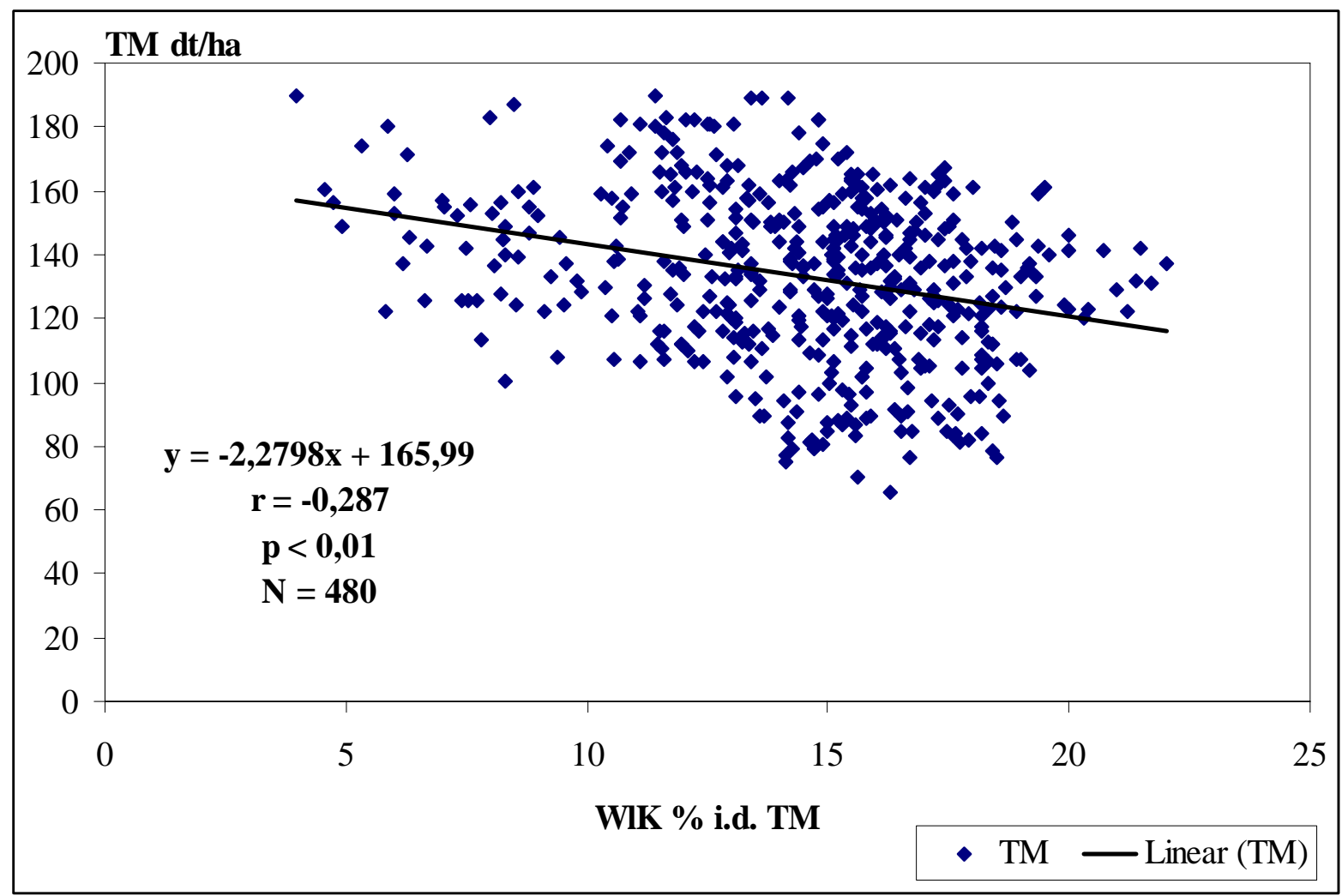

Abb. 36: Wasserlösliche Kohlenhydrate (WlK) in Korrelation zu dem TM-Ertrag im Gras.

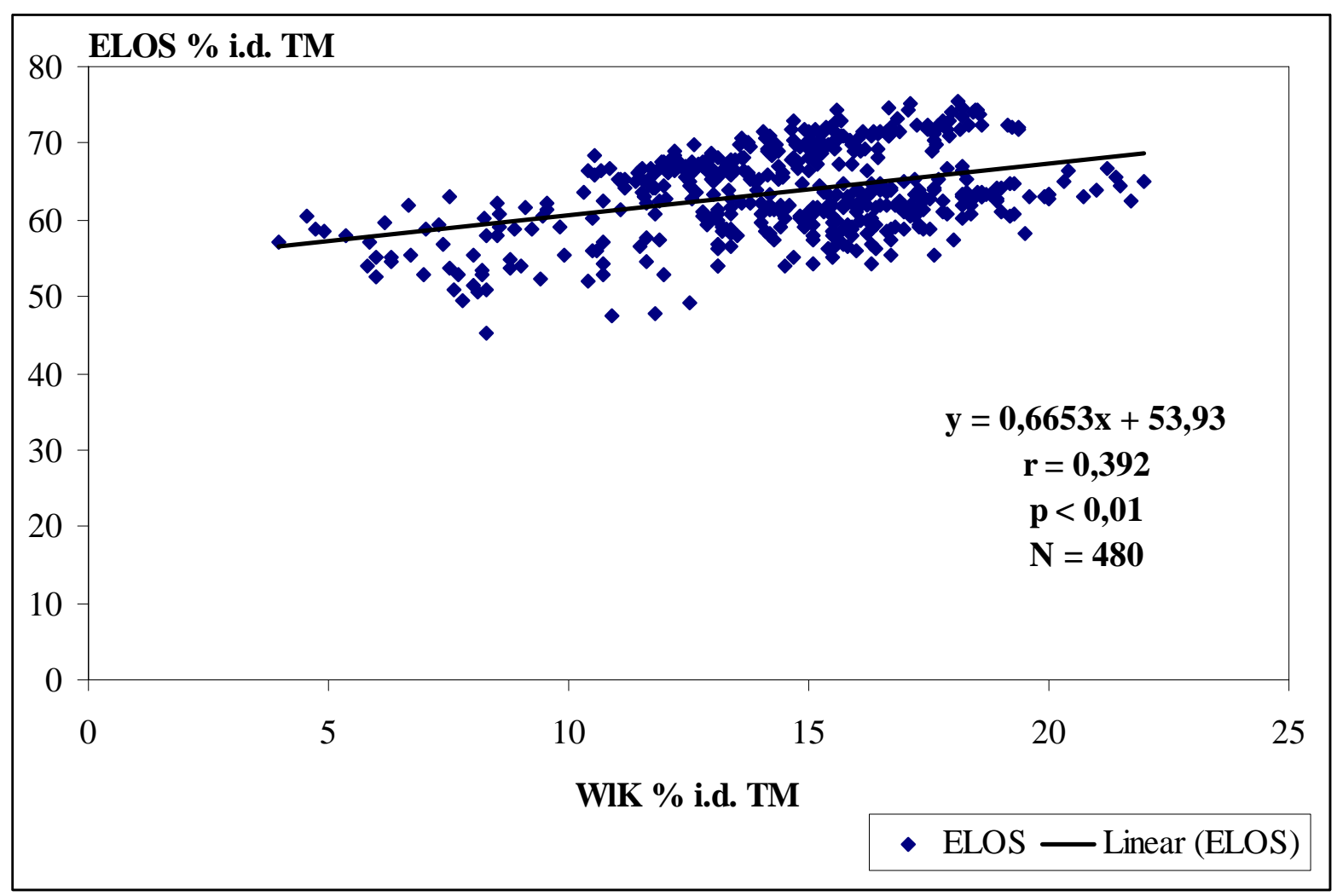

Abb. 37: Wasserlösliche Kohlenhydrate (WlK) in Korrelation zu der Verdaulichkeit (ELOS) im Gras. 


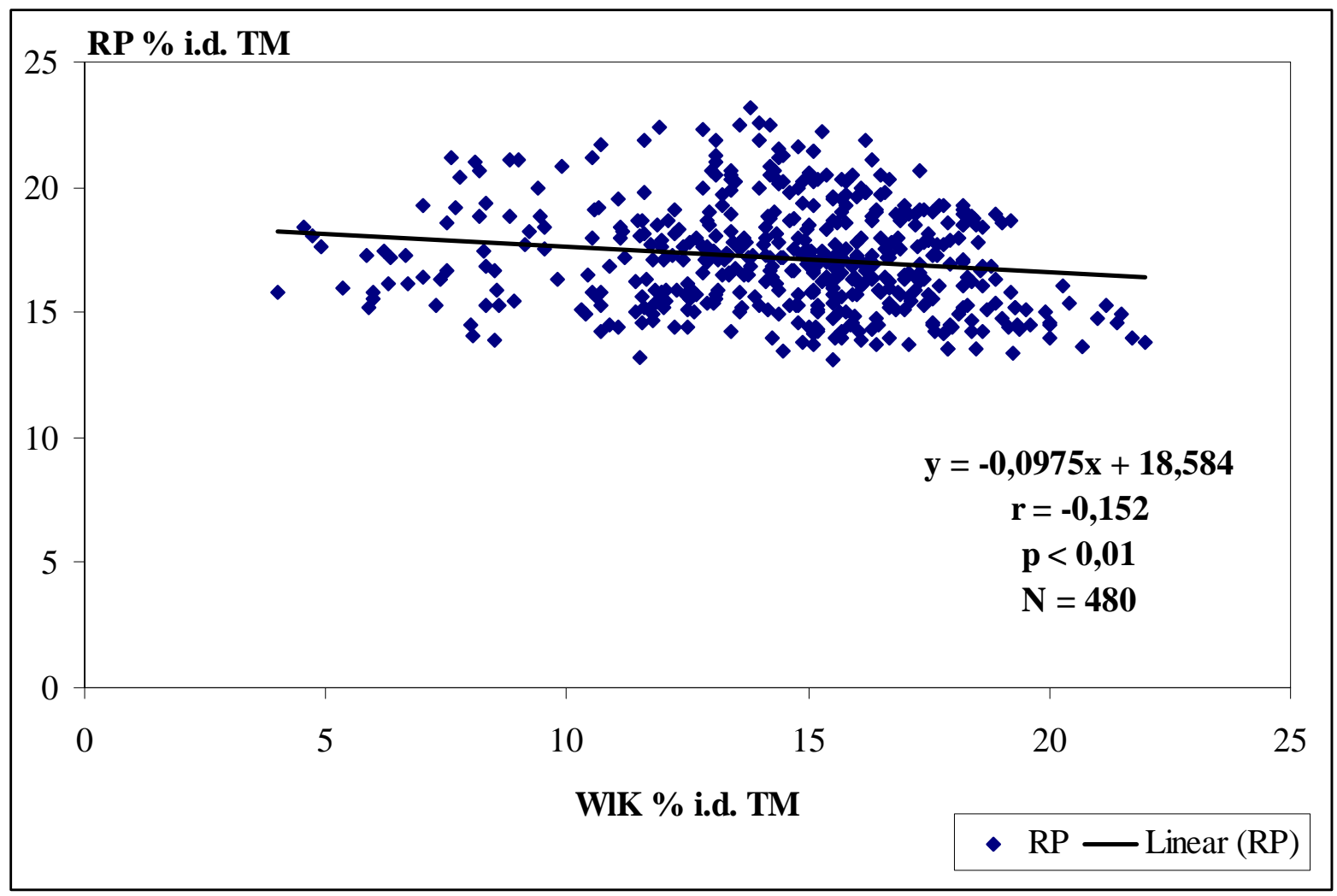

Abb. 38: Wasserlösliche Kohlenhydrate (WlK) in Korrelation zu dem Rohproteingehalt (RP) im Gras.

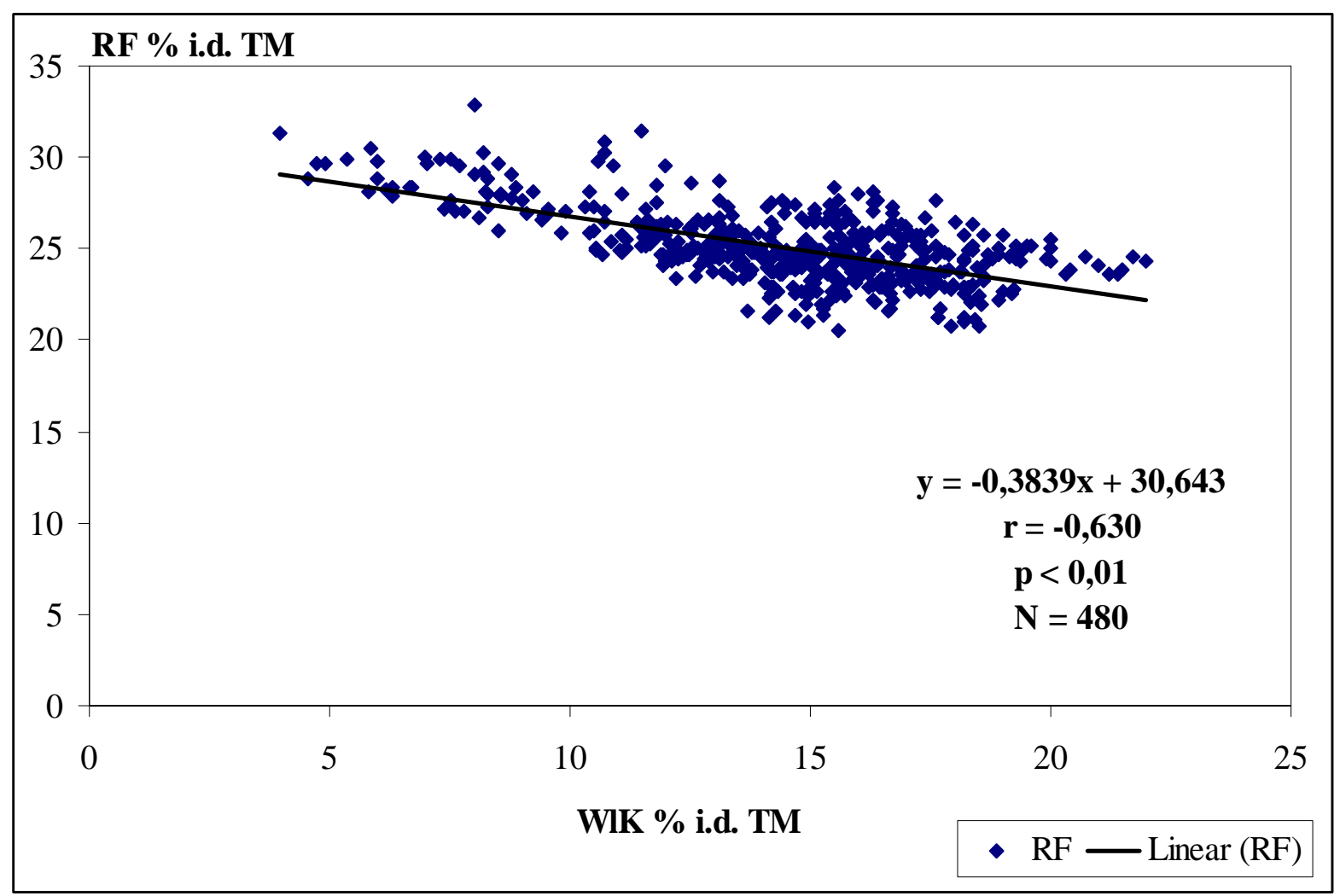

Abb. 39: Wasserlösliche Kohlenhydrate (WlK) in Korrelation zu dem Rohfasergehalt (RF) im Gras. 


\subsection{Ergebnisse Zeitreihenversuch}

Die wasserlöslichen Kohlenhydrate (WlK) und der Fruktangehalt wiesen im Verlauf des 1. Aufwuchses im Mittel der Sorten und der allen Ernten bei demselben phänologischen Stadium keine signifikanten Unterschiede zwischen 1HVJ und 2HVJ auf (Tab. 33).

Tab. 33: Wasserlösliche Kohlenhydrate (WlK) \% i.d. TM und Fruktangehalt \% i.d. TM im Verlauf des 1. Aufwuchses des $1 \mathrm{HVJ}$ und des 2HVJ über alle fünf Sorten und im Mittel aller Ernten bei demselben phänologischen Stadium.

\begin{tabular}{|c|c|c|c|c|c|c|}
\hline Versuchsjahr & WlK & N & SD & Fruktan & N & SD \\
\hline 1HVJ (2006) & 24,4 a & 20 & 5,10 & 15,9 a & 20 & 5,28 \\
\hline 2HVJ (2007) & 25,3 a & 20 & 2,36 & 16,3 a & 20 & 2,76 \\
\hline
\end{tabular}

Signifikante Unterschiede gelten für Werte in derselben Spalte mit unterschiedlichen Buchstaben bei $\mathrm{P}<0,05$, $\mathrm{SD}=$ Standardabweichung

\subsubsection{Wasserlösliche Kohlenhydrate und Fruktangehalt im Verlauf des ersten Auf- wuchses}

Bei der Gemischprobe der Sorte Respect stiegen die WlK und der Fruktangehalt im Verlauf des 1. Aufwuchses bis zur 8. Ernte $(\mathrm{BBCH}=51$, Ährenschieben (Tab. 34)) an, danach sanken sie bis zum Ende des Aufwuchses ab, bei der Sorte Licarta stiegen die WlK und der Fruktangehalt bis zur 7. Ernte dann sanken sie in der 8. Ernte ab dann stiegen sie in 9. Ernte (BBCH = 51) wieder ab danach sanken sie bis zum Ende des Aufwuchses ab. Bei Sorten EGB1, Rodrigo und AberAvon stiegen die WlK und der Fruktangehalt bis zur 9. Ernte $(\mathrm{BBCH}=51)$ an, danach sanken sie bis zum Ende des Aufwuchses ab. Das führte dazu, dass die WlK und der Fruktangehalt zunächst bis kurz vor Beginn des Ährenschiebens zunahmen, dann sie bis zum Ende des Aufwuchses abfielen (Abb. 40).

Beim Stängel sank die WlK bei den Sorten Respect und Licarta von der 6. zu der 8. Ernte ab. Dagegen stieg die WlK bei den Sorten EGB1 und Rodrigo von der 6. zu der 7. Ernte an, sank dann sie von der 7. zur 8. Ernte ab. Dann stieg sie bei den genannten vier Sorten von der 8. zur 9. Ernte stark an. Bei der Sorte AberAvon stieg die WlK bis zu 9. Ernte leicht an. Von der 9. Ernte bis zum Ende des Aufwuchses sank die WlK sehr stark bei allen Sorten ab.

Der Frukantgehalt stieg bei allen Sorten von der 6. zur 7. Ernte an dann sank er von der 7. zur 8. Ernte ab. Von der 8. zur 9. Ernte stieg er wieder an (mit Ausnahme der Sorte EGB1: der Fruktangehalt sank von der 7. zur 9. Ernte). Von der 9. Ernte bis zum Ende des Aufwuchses sank der Fruktangehalt bei allen Sorten sehr stark. D.h., dass die WlK und der Fruktangehalt am Anfang des Aufwuchses hoch waren, danach sanken sie bis zur 9. Ernte. In der 9. Ernte 
waren die WlK und der Fruktangehalt wieder hoch, von der 9. Ernte bis zum Ende des Aufwuchses sanken beide parameter sehr stark (Abb. 41).

Bei den Blättern stieg die WIK bei den Sorten Respect und EGB1 von der 6. zur 7. Ernte, dagegen sank sie bei den Sorten Licarta, Rodrigo und AberAvon. Von der 7. zur 8. Ernte sank die WlK bei allen Sorten ab, von der 8. zur 9. Ernte stieg sie an. Der Fruktangehalt sank von der 9. Ernte bis zum Ende des Aufwuchses sehr bei allen Sorten stark (Abb. 42).

Tab.34: Die Phänologischen Stadien (BBCH) der Deutschen Weidelgrassorten zu den Erntenterminen des 1HVJ und 2. HVJ des Zeitreihenversuches.

\begin{tabular}{|c|c|c|c|c|c|c|}
\hline \multirow{3}{*}{ Ernte } & \multirow{3}{*}{ Datum } & \multicolumn{5}{|c|}{ BBCH - Code* } \\
\hline & & \multicolumn{5}{|c|}{ Sorten } \\
\hline & & Respect & Licarta & EGB1 & Rodrigo & AberAvon \\
\hline \multicolumn{7}{|l|}{2006} \\
\hline 1. Ernte & 03.05 & 31 & 31 & 31 & 31 & 30 \\
\hline 2. Ernte & 10.05 & 32 & 32 & 32 & 32 & 31 \\
\hline 3. Ernte & 17.05 & 34 & 34 & 34 & 34 & 32 \\
\hline 4. Ernte & 24.05 & 51 & 51 & 51 & 51 & 47 \\
\hline \multicolumn{7}{|l|}{2007} \\
\hline 1. Ernte & 12.04 & 29 & 29 & 29 & 29 & 29 \\
\hline 2. Ernte & 16.04 & 29 & 29 & 29 & 29 & 29 \\
\hline 3. Ernte & 19.04 & 31 & 29 & 29 & 29 & 29 \\
\hline 4. Ernte & 25.04 & 32 & 31 & 31 & 31 & 31 \\
\hline 5. Ernte & 28.04 & 32 & 31 & 31 & 31 & 31 \\
\hline 6. Ernte & 02.05 & 33 & 31 & 32 & 32 & 31 \\
\hline 7. Ernte & 06.05 & 34 & 32 & 33 & 33 & 32 \\
\hline 8. Ernte & 13.05 & 51 & 49 & 51 & 49 & 45 \\
\hline 9. Ernte & 16.05 & 51 & 51 & 51 & 51 & 47 \\
\hline 10. Ernte & 24.05 & 61 & 61 & 61 & 59 & 51 \\
\hline 11. Ernte & 30.05 & 65 & 63 & 63 & 63 & 55 \\
\hline
\end{tabular}

* Nach Stauss et al. (1994): Einheitliche Codierung der phänologischen Entwicklungsstadien mono- und dikotyler Pflanzen. Erweiterte BBCH-Skala: Allgemein. Ciba-Geigy AG, Basel, 58 S. 


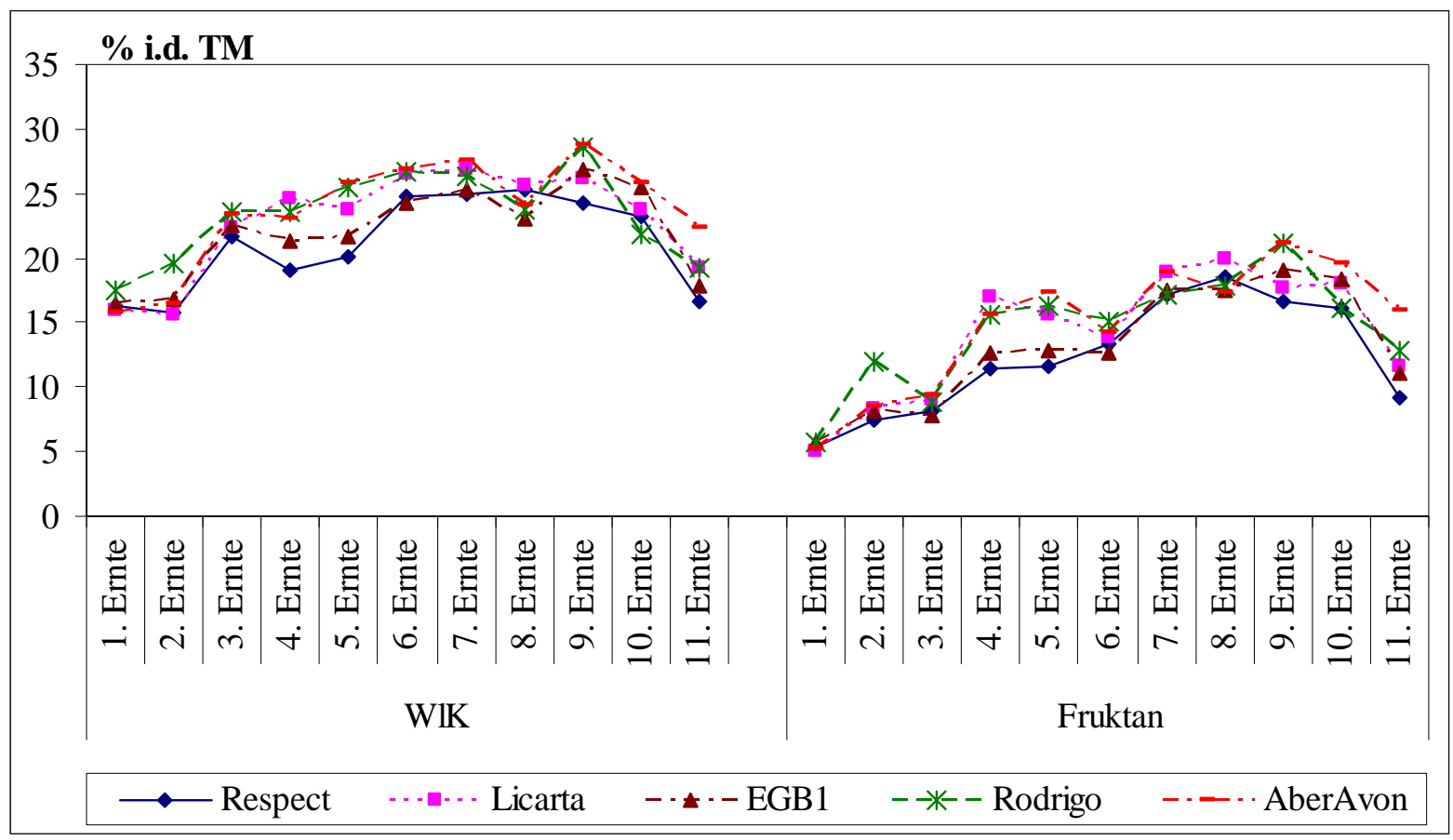

Abb. 40: Wasserlösliche Kohlenhydrate und Fruktangehalt der Gemischprobe im Verlauf des ersten Aufwuchses für alle Sorten im 2HVJ.

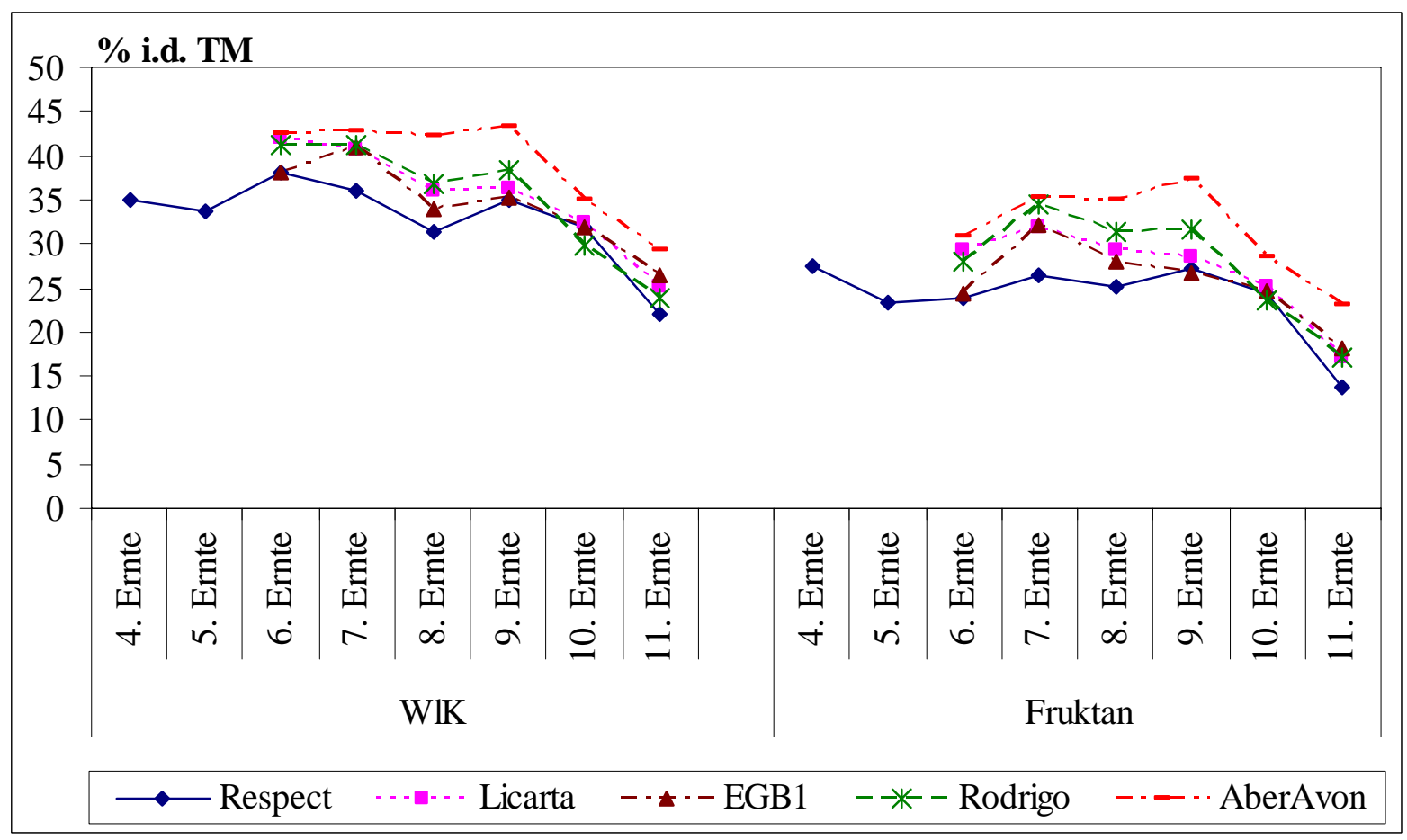

Abb. 41: Wasserlösliche Kohlenhydrate und Fruktangehalt des Stängels im Verlauf des ersten Aufwuchses für alle Sorten im 2HVJ. 


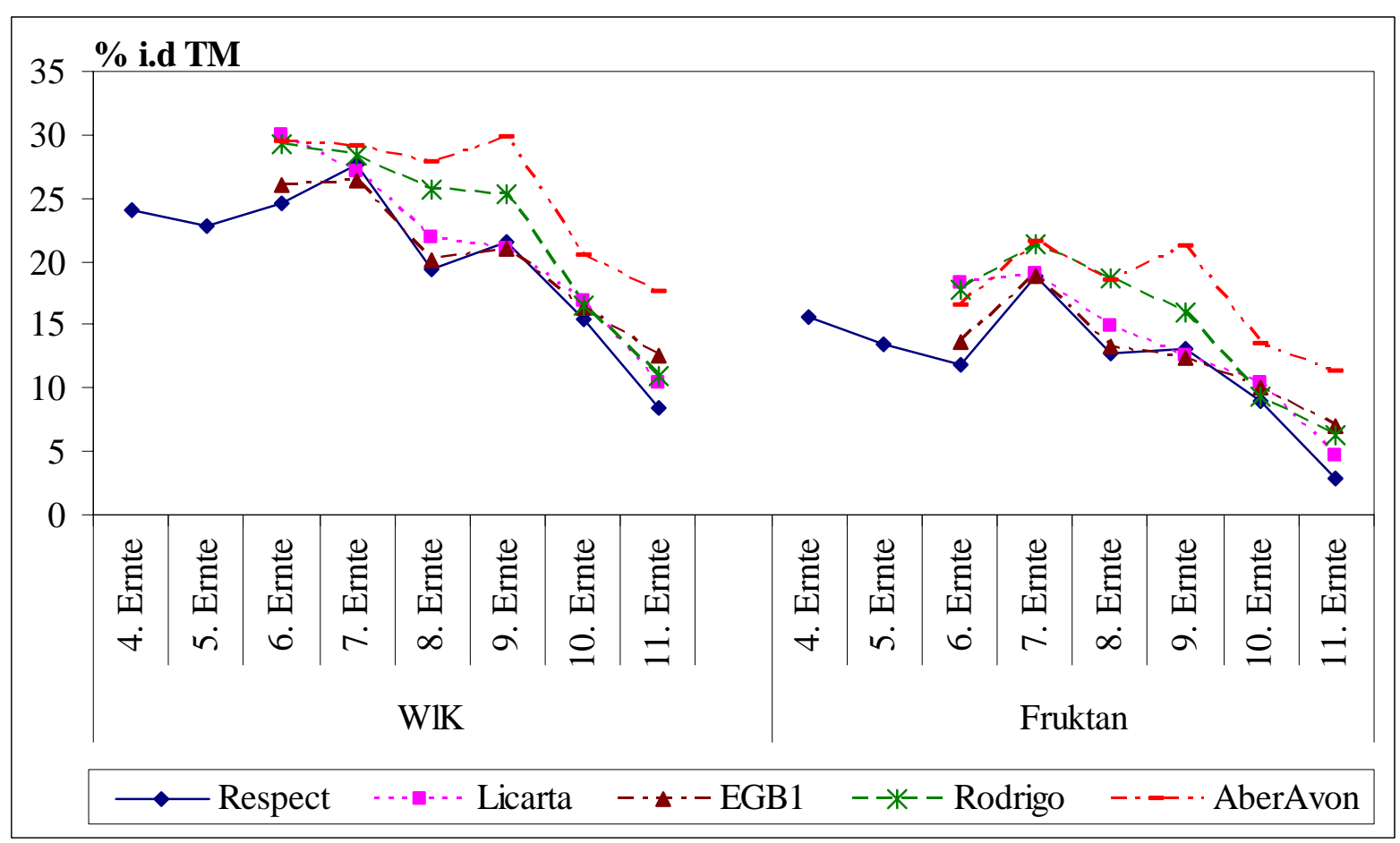

Abb. 42: Wasserlösliche Kohlenhydrate und Fruktangehalt der Blätter im Verlauf des ersten Aufwuchses für alle Sorten im 2HVJ.

\subsubsection{Sortenunterschiede}

Die Deutsche Weidelgrassorten erreichten im Verflauf des ersten Aufwuchses im Mittel aller Ernten und der beiden Versuchsjahre (1HVJ und 2HVJ) in der Gemischprobe einen durchschnittlichen Fruktangehalt von 13,64 \% i.d. TM und einen durchschnittlichen Gehalt der WlK von 22,36 \% i.d. TM. Überduchschnittlich hohe Gehalte erreichten die Sorten AberAvon, Rodrigo und Licarta. Die Sorte AberAvon wies die höchsten WlK und Fruktangehalt auf. Den tendenziell niedrigsten Gehalt an WlK und Fruktan hatte die Sorte Respect (Abb. 43).

Bei jeder Ernte im 2HVJ im Stängel und in den Blättern wies AberAvon den höchsten WlK und Fruktangehalt auf (Abb. 41 und 42).

\subsubsection{Wasserlösliche Kohlenhydrate und Fruktangehalt in den Grasfraktionen}

Die WlK und der Fruktangehalt nahmen in der Reihenfolge Stängel, Blätter und Ähren ab (Abb. 44). Der prozentuale Fruktangehalt in den WlK war bei den Ähren niedriger als bei den Blättern und diese wiederum niedriger als im Stängel (Abb. 44). 


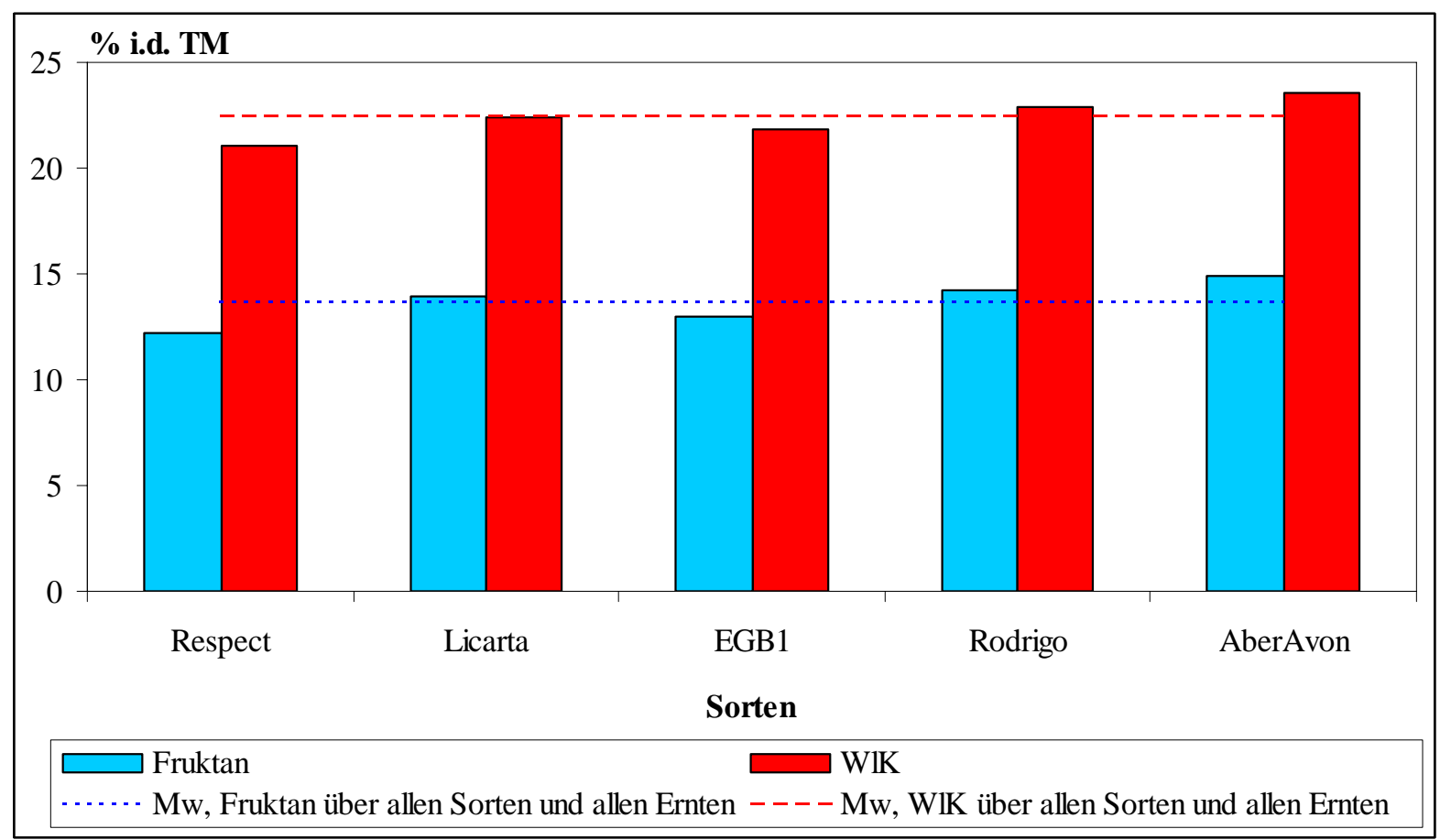

Abb. 43: Wasserlösliche Kohlenhydrate und Fruktangehalt in verschiedenen Deutschem Weidelgrassorten im Verflauf des ersten Aufwuchses, im Mittel der allen Ernten und der beiden Versuchsjahren (1HVJ und 2HVJ).

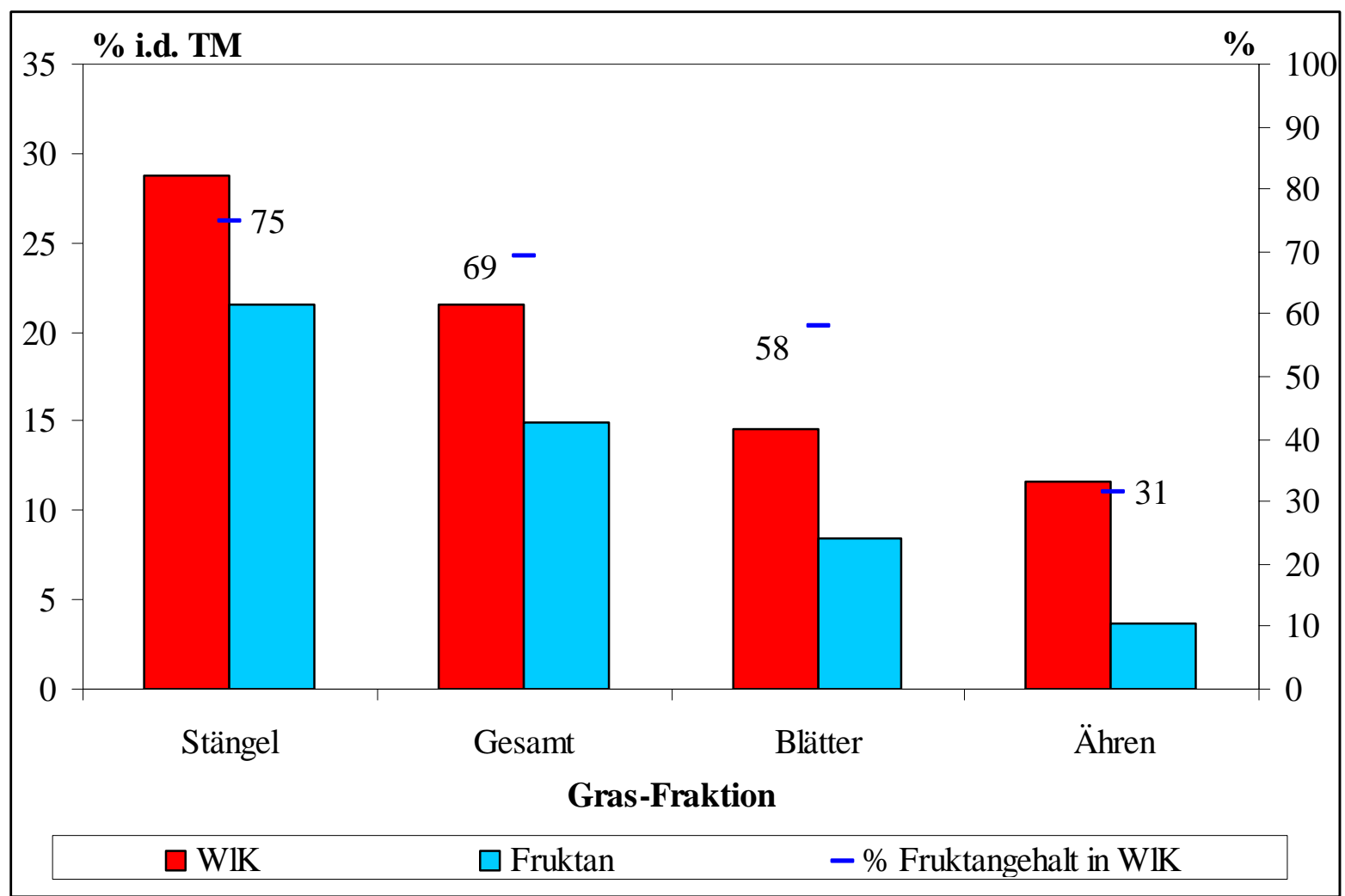

Abb. 44: Wasserlösliche Kohlenhydrate, Fruktangehalt und Fruktangehalt (\%) in den WlK in der Grasfraktion, im Sortenmittel Mittelwert aus der 10. und 11. Ernte im 2HVJ. 


\subsubsection{Tageszeiteneinfluss auf WIK und Fruktangehalt}

Im Verlauf des 1. Aufwuchses wurden die Proben im 1HVJ (2006) ein Mal wöchentlich zwei Mal im Tag (8 und 13 Uhr). Die WlK wiesen im Mittel der Sorten, der Ernten und der NDüngungsstufen keine signifikanten Unterschiede zwischen Tageszeiten (8 und 13 Uhr) auf. Dagegen war der Fruktangehalt um 8 Uhr höher als um 13 Uhr (Tab. 35).

Tab. 35: Wasserlösliche Kohlenhydrate (WlK) \% i.d. TM und Fruktangehalt \% i.d. TM im Verlauf des 1. Aufwuchses des 1HVJ in Abhängigkeit von Tageszeiten (8 Uhr und 13 Uhr) im Mittel der fünf Sorten, aller Ernten und der beiden N-Düngungsstufen (normal und niedrig).

\begin{tabular}{|c|c|c|c|c|c|c|}
\hline Tageszeiten & WlK & N & SD & Fruktan & N & SD \\
\hline $8 \mathrm{Uhr}$ & $28,8 \mathrm{a}$ & 40 & 6,7 & $21,4 \mathrm{a}$ & 40 & 7,8 \\
\hline $13 \mathrm{Uhr}$ & $29,0 \mathrm{a}$ & 40 & 5,9 & $19,8 \mathrm{~b}$ & 40 & 7,1 \\
\hline
\end{tabular}

Signifikante Unterschiede gelten für Werte in derselben Spalte mit unterschiedlichen Buchstaben bei $\mathrm{P}<0,05$, $\mathrm{SD}=$ Standardabweichung.

\subsubsection{Temperatureinfluss auf WlK und Fruktangehalt}

Im Mittel der Sorten und der beiden Versuchsjahre (1HVJ und 2HVJ) war der Zusammenhang zwischen dem wasserlöslichen Kohlenhydrate-Gehalt und der Temperatur nicht signifikant (Abb. 45). Ebenso gab es keinen signifikanten Zusammenhang zwischen dem Fruktangehalt und der Temperatur (Abb. 46).

\subsubsection{N-Düngungseinfluss auf WlK und Fruktangehalt}

Im Verlauf des 1. Aufwuchses des 1HVJ (2006) führte die höhere N-Düngungsstufe im Mittel der Sorten und der Ernten zu einer Abnahme der WlK (Tab. 36). Auch Fruktangehalt und NDüngungsstufe korrelieren negativ miteinander, wobei steigende Fruktangehalte im Gras von niedriger N-Düngungsstufe begleitet werden (Tab. 36).

Tab. 36: Wasserlösliche Kohlenhydrate (WlK) \% i.d. TM und Fruktangehalt \% i.d. TM im Verlauf des 1. Aufwuchses des $1 \mathrm{HVJ}$ in Abhängigkeit von N-Düngungsstufen (normal und niedrig) im Mittel der fünf Sorten, aller Ernten und der beiden Tageszeiten (8 Uhr und 13 Uhr).

\begin{tabular}{|c|c|c|c|c|c|c|}
\hline N-Düngungsstufen & WlK & $\mathrm{N}$ & $\mathrm{SD}$ & Fruktan & $\mathrm{N}$ & $\mathrm{SD}$ \\
\hline Normaldüngung & $24,6 \mathrm{~b}$ & 40 & 4,7 & $15,3 \mathrm{~b}$ & 40 & 5,0 \\
\hline Niedrigdüngung & $33,3 \mathrm{a}$ & 40 & 4,5 & $26,0 \mathrm{a}$ & 40 & 5,4 \\
\hline
\end{tabular}

Signifikante Unterschiede gelten für Werte in derselben Spalte mit unterschiedlichen Buchstaben bei $\mathrm{P}<0,05$, SD = Standardabweichung. 


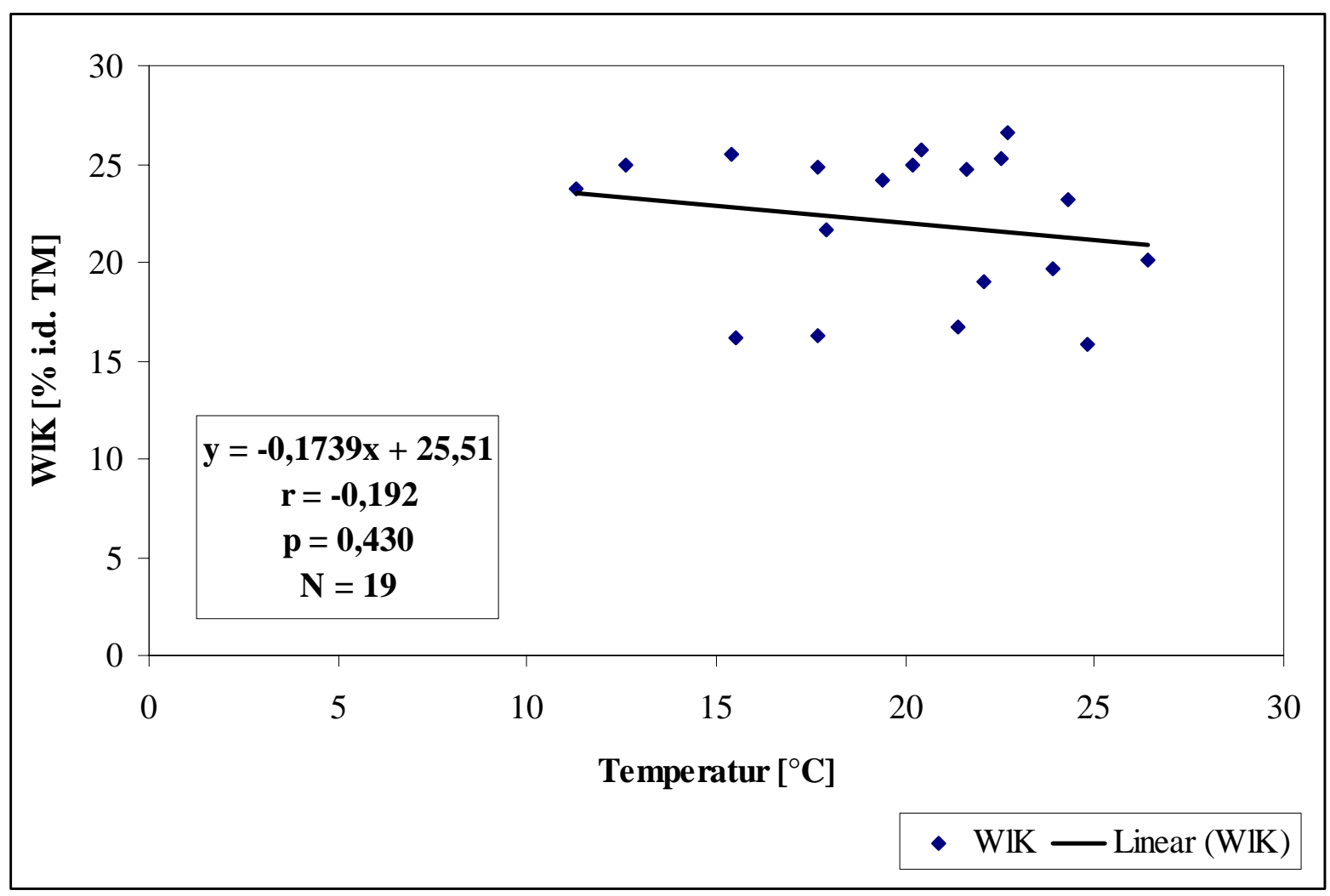

Abb. 45: Wasserlösliche Kohlenhydrate (WlK) in Korrelation zur Temperatur $\left({ }^{\circ} \mathrm{C}\right)$ im Mittel der Sorten und der beiden Versuchsjahre (1HVJ und 2HVJ).

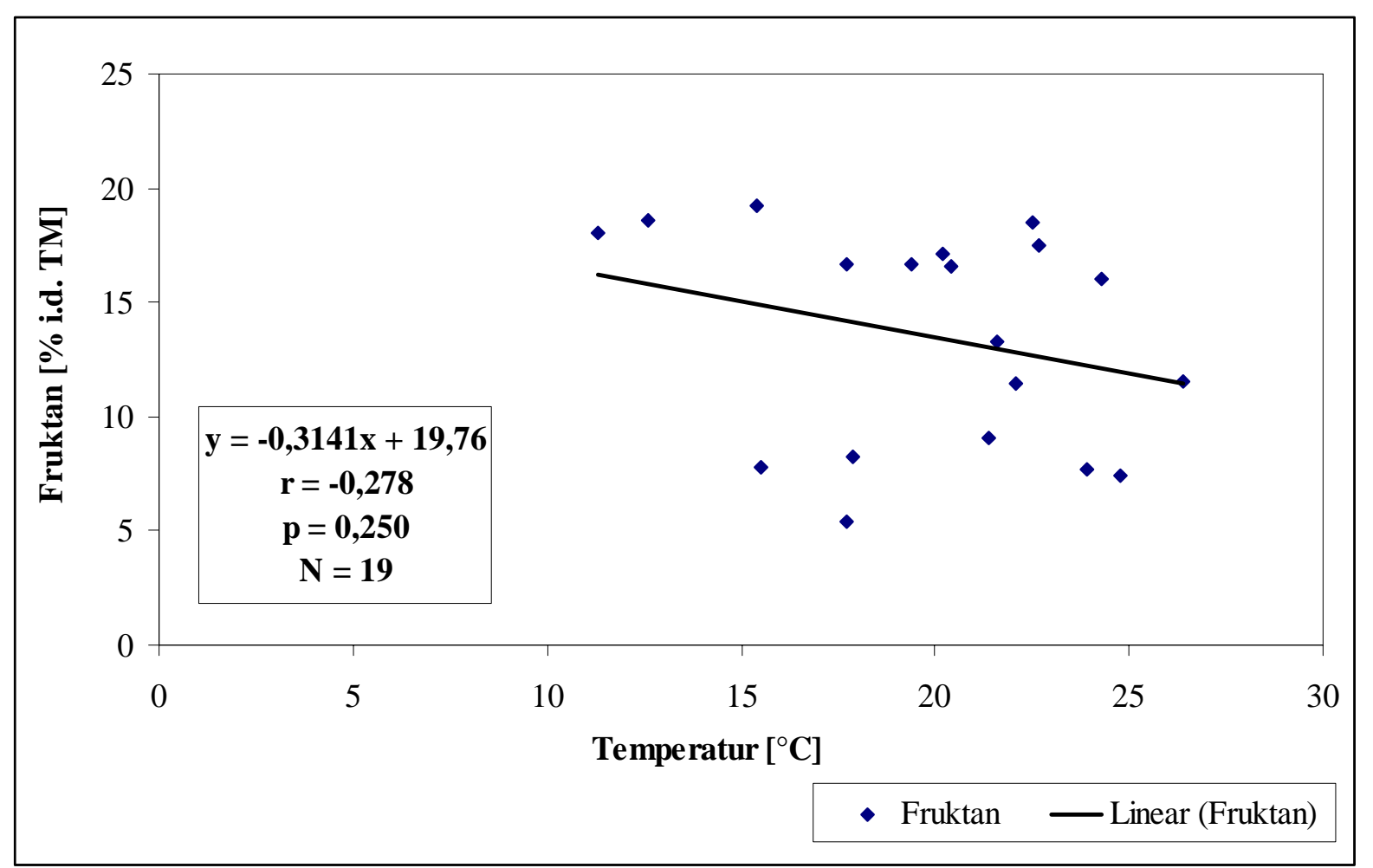

Abb. 46: Fruktangehalt in Korrelation zur Temperatur $\left({ }^{\circ} \mathrm{C}\right)$ im Mittel der Sorten und der beiden Versuchsjahre (1HVJ und 2HVJ). 


\subsubsection{Verteilung des Fruktangehaltes nach dem Polymerisationsgrad}

In den Tabellen (A2 bis A6) sind die Werte des Polymerisationsgrades für die fünf Weidelgräser entsprechend den elf Terminen in der Gesamtprobe, der Blatt- und der Stängelfraktion aufgeführt. Die analysierten Weidelgräser weisen einen Polymerisationsgrad der Fruktane von bis zu 60 Kettenlängen auf. Der mittlere Polymerisationsgrad (DP) der akkumulierten Fruktane differenzierte sich in allen fünf Sorten. Um entsprechende Aussagen abzuleiten, ist der Polymerisationsgrad in Einheiten von zehn Peaks zusammengefasst worden. In den Abbildungen 50 bis 55 sind die Daten graphisch dargestellt. Zum Vergleich zwischen hohem und niedrigem WlK - Gehalt bei Deutschen Weidelgrassorten wurden die Sorten AberAvon (Hoch-Zucker-Gras) und Respect ausgewählt. Diese beiden Sorten wiesen über alle gemessenen Termine ein niedriges (Respect) und ein hohes Niveau (AberAvon) an WlK und Fruktanen auf (Abb. 17 und 18).

Generell lässt sich eine Dynamik hinsichtlich des Polymerisationsgrades feststellen, die sich dadurch auszeichnet, dass sich mit zunehmender Entwicklung die niederpolymeren Ketten abbauen und die höherpolymeren Ketten aufgebaut werden (Abb. 47 und 48). Die Sorte AberAvon weist tendenziell höhere Anteile an niederpolymeren Ketten über die gesamte Untersuchungszeit auf als die Sorte Respect. Besonders mit zunehmender Reife, ab dem 7. Termin (optimaler Erntezeitpunkt für Respect nach Entwicklungsstadium) nehmen die mittelund längerkettigen Polymere bei Respect deutlich zu. Lediglich der 9. Termin zeigt hier eine kleine Ausnahme, wo die erste Polymerfraktion wieder verhältnismäßig angestiegen ist. Bei der Sorte AberAvon ist der gleiche Verlauf bis zum 7. Termin feststellbar, allerdings bleibt der verhältnismäßige Abstand zwischen den ersten polymeren Kettenlängen und den mittelund längerkettigen Strukturen erheblich größer. Ab dem 8. Termin (optimaler Erntezeitpunkt für AberAvon nach Entwicklungsstadium) nehmen die niederploymeren Kettenlängen wieder zu. Diese Unterschiede zwischen beiden Sorten waren in den fraktionierten Pflanzenteilen den Blättern und den Stängeln besonders ausgeprägt. Zunächst liegt vom 4. zum 6. Termin in den Blättern (Abb. 49 und 50) der Anteil der kurzkettigen Polymerisationsgrade bei beiden Sorten zwischen $40 \%$ und 50 \%. Bei Respect reduziert er sich auf ca. 35 \% (7. - 10. Termin); zum 11. Termin erhöhte er sich wieder auf über 50 \%. Von der 4. Ernte stieg der 2. Polymerisationsgrad deutlich an bis auf über $30 \%$ zum 8. Erntetermin. Ab diesem Zeitpunkt fiel er dann leicht ab. Der 3. und die weiteren Polymerisationsgrade erhöhten sich ebenfalls kontinuierlich. Bei der Sorte AberAvon waren diese Verläufe nicht so gegeben. Der 1. Polymerisationsgrad repräsentierte in allen Ernteterminen den höchsten Anteil mit ca. 40 \%. Die anderen Polymerisationsketten blieben über alle Termine auf einem vergleichbaren Niveau. Lediglich 
der 10. Erntetermin bildete eine Ausnahme, wo auf über $20 \%$ gestiegener Anteil der 3. Kettenfraktion festgestellt wurde. Für die Stängelfraktion (Abb. 51 und 52) kann das für Blätter gesagte ebenfalls tendenziell nachvollzogen werden. Im Unterschied zu der Blattfraktion sind wesentlich höhere Anteil der 1. Polymerisationskette in beiden Sorten feststellbar. Insgesamt weist AberAvon höhere Anteile dieser Fraktion auf. Im Entwicklungsverlauf stiegen die mittel- und langkettigen Polymerisationsanteile bei der Sorte Respect stärker an als bei der Sorte AberAvon. Beispielsweise überflügelt bei der Sorte Respect zum 11. Erntetermin die 2. Polymerisationskette die Werte der 1. Fraktion. Zu diesem Stadium hatte sich bereits eine Ähre gebildet. 


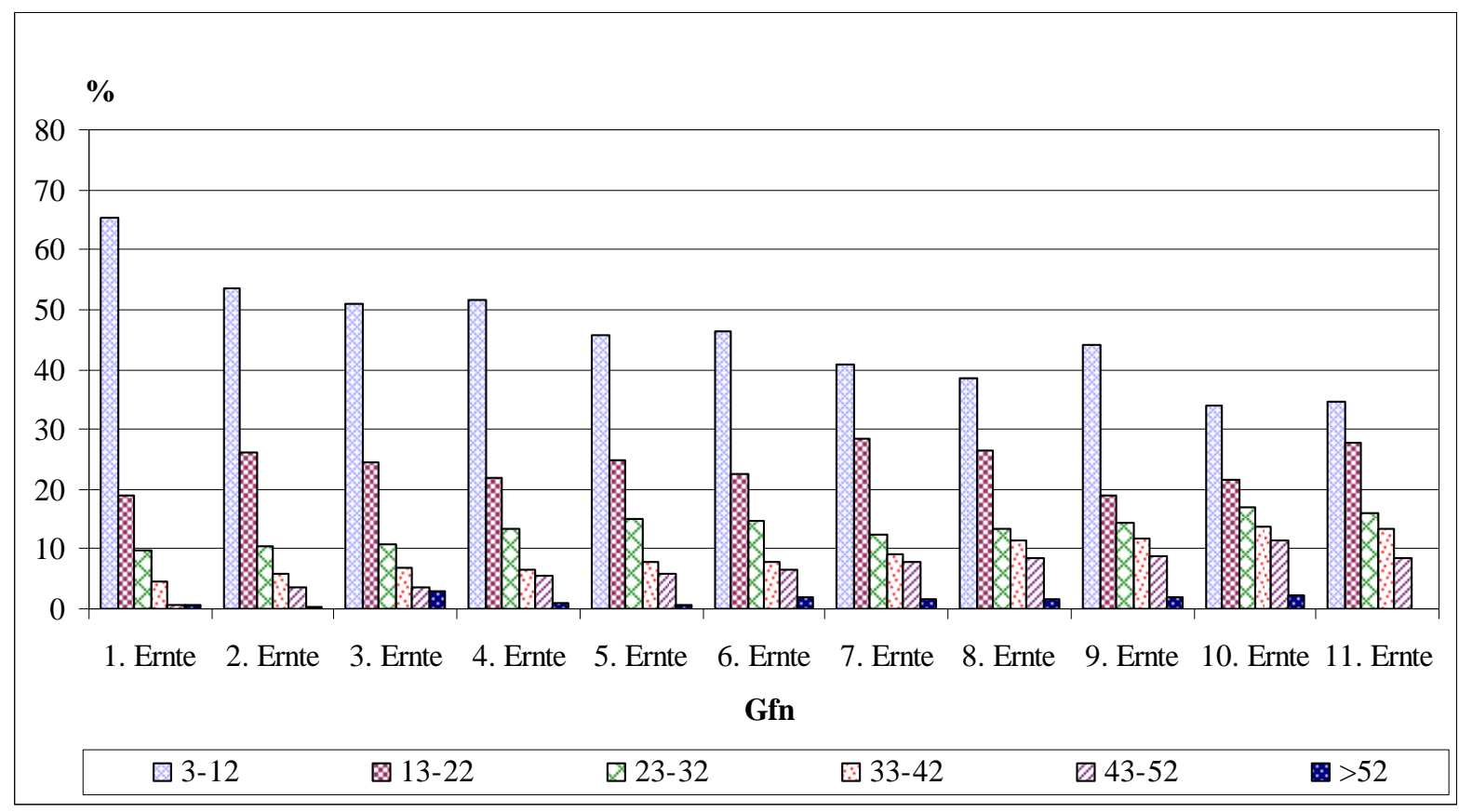

Abb. 47: Verteilung des Fruktangehaltes (\%) nach dem Polymerisationsgrad für die Sorte Respect in der Gemischprobe im 2HVJ (2007) in allen Ernteterminen.

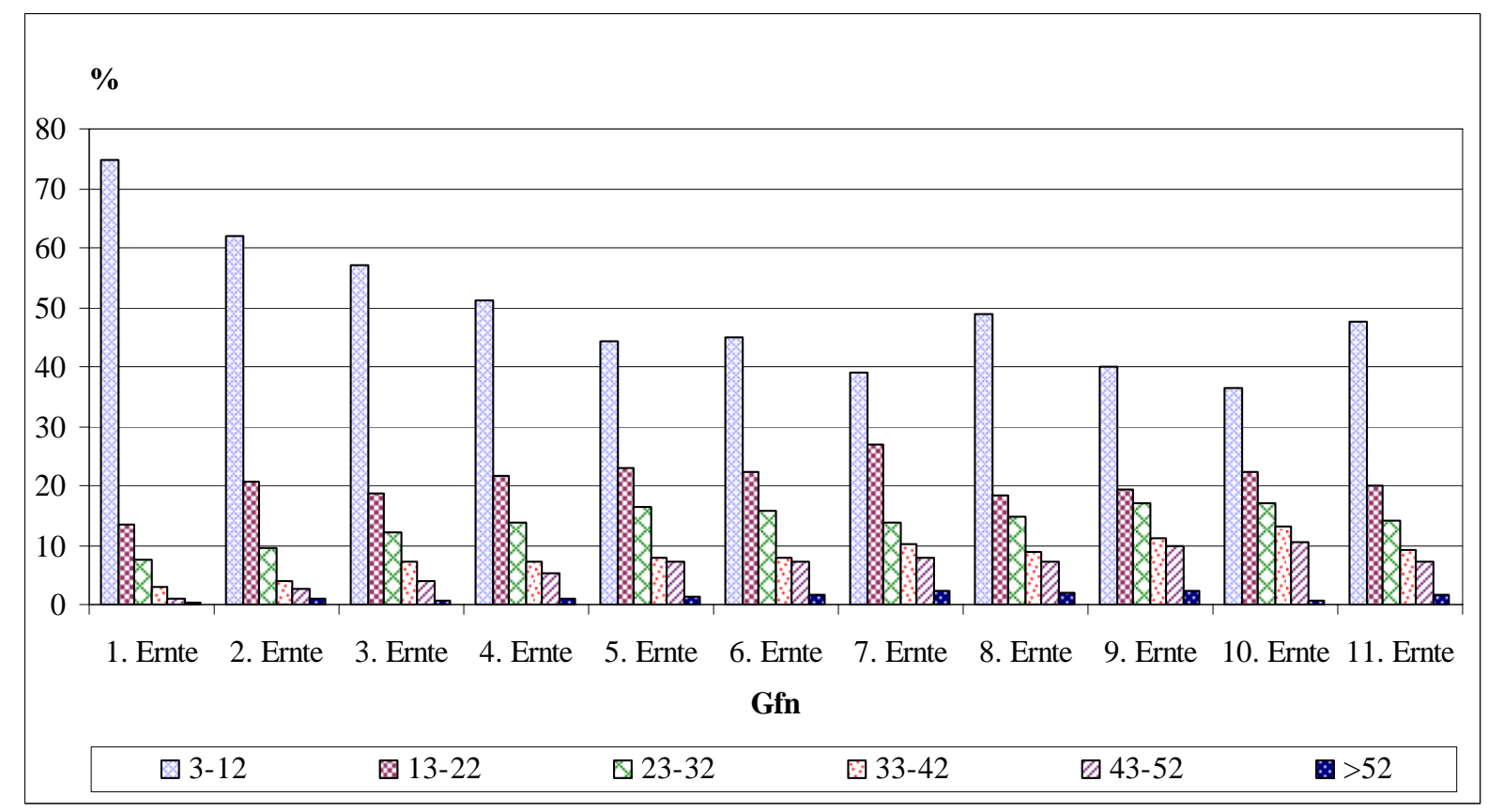

Abb. 48: Verteilung des Fruktangehaltes (\%) nach dem Polymerisationsgrad für die Sorte AberAvon in der Gemischprobe im 2HVJ (2007) in allen Ernteterminen. 


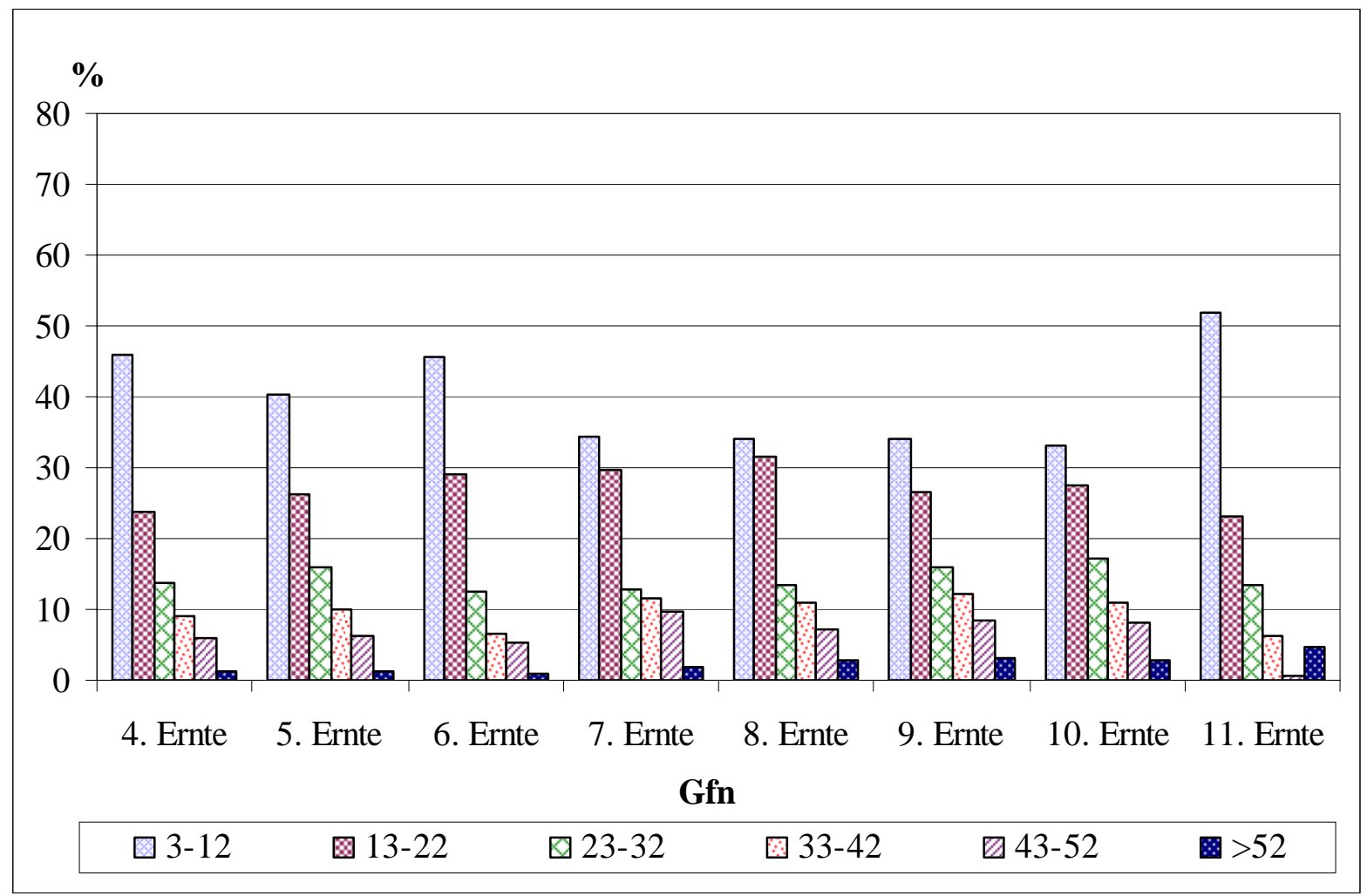

Abb. 49: Verteilung des Fruktangehaltes (\%) nach dem Polymerisationsgrad für die Sorte Respect in den Blättern im 2HVJ (2007) in allen Erntenterminen.

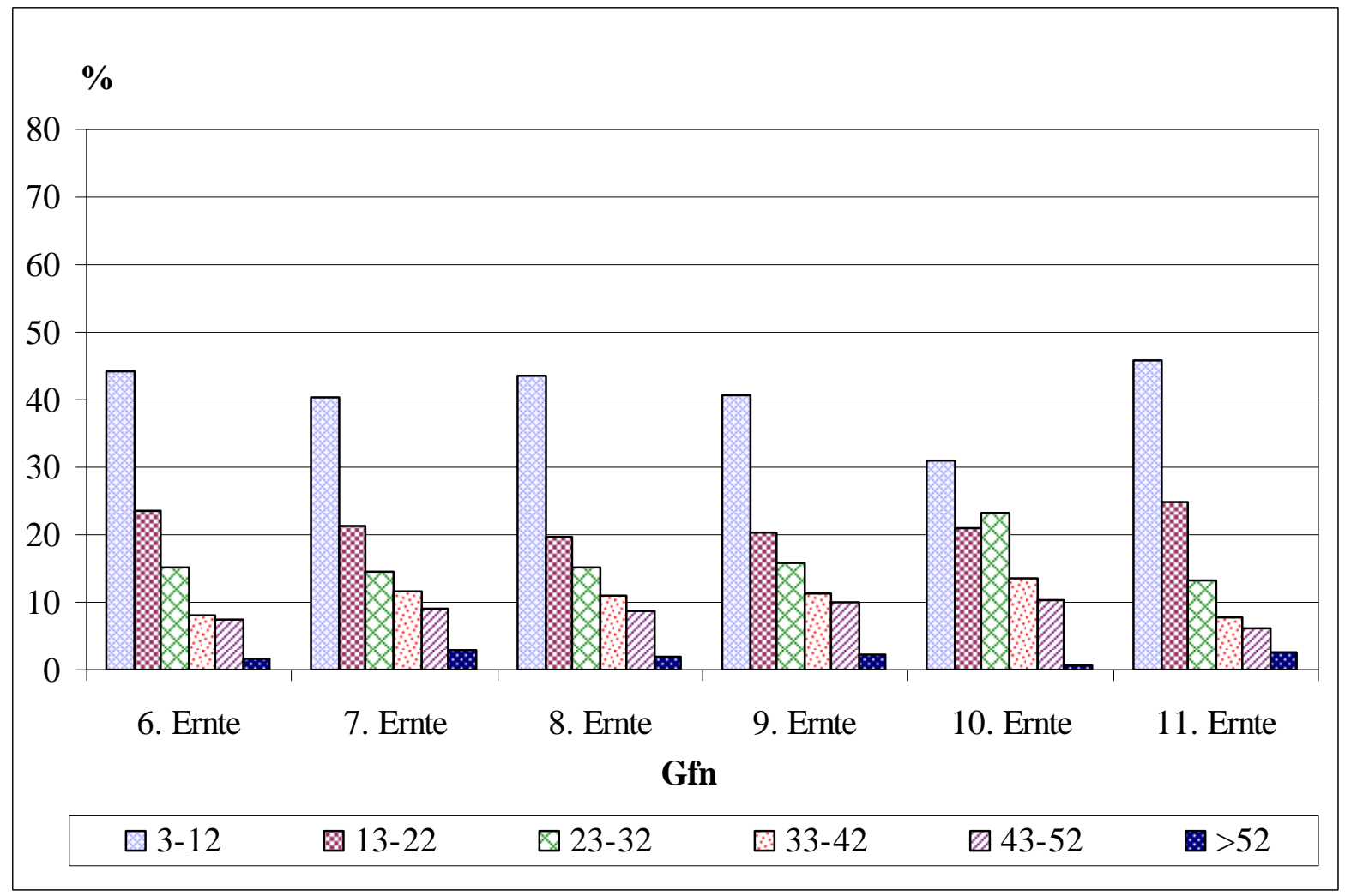

Abb. 50: Verteilung des Fruktangehaltes (\%) nach dem Polymerisationsgrad für die Sorte AberAvon in den Blättern im 2HVJ (2007) in allen Ernteterminen. 


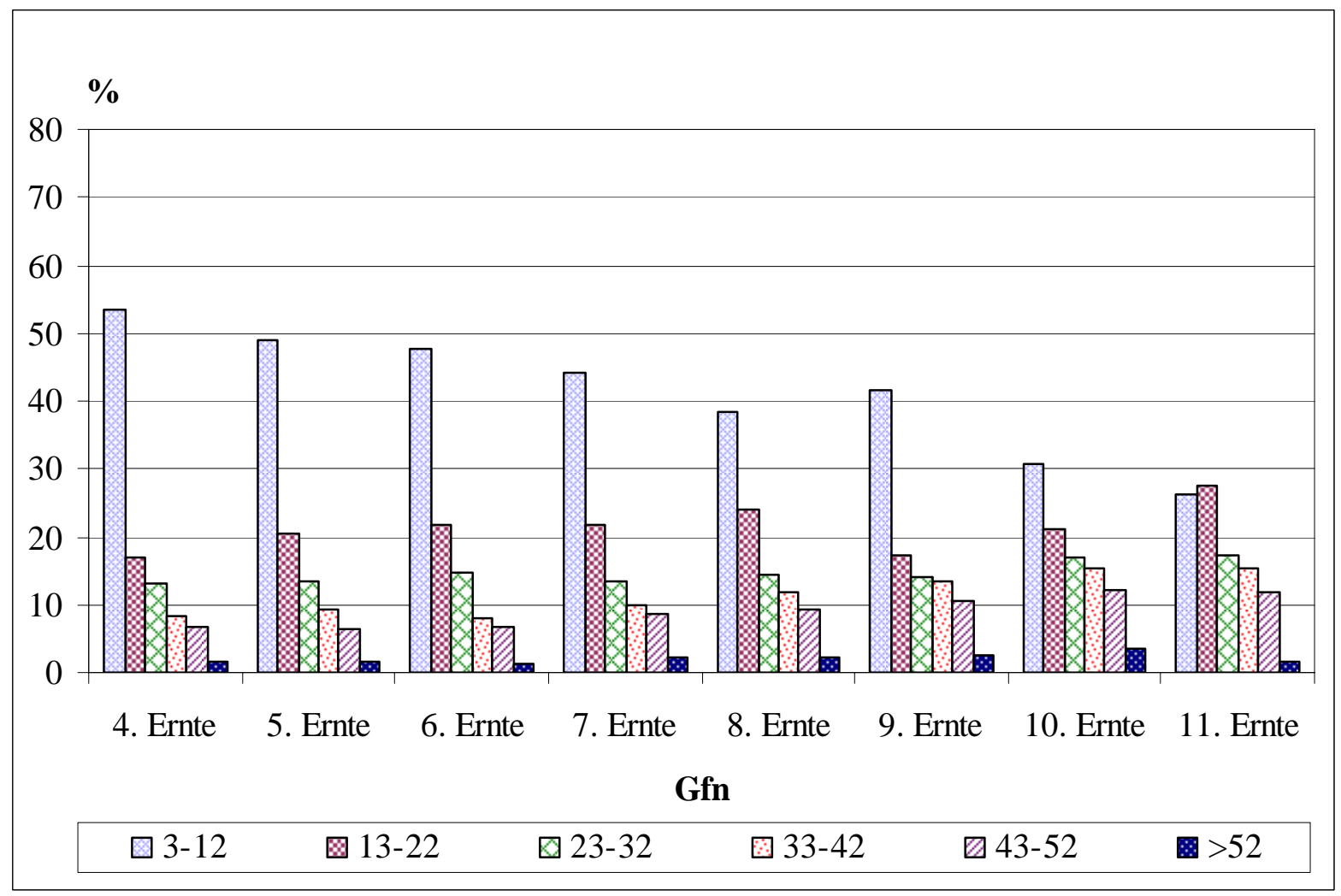

Abb. 51: Verteilung des Fruktangehaltes (\%) nach dem Polymerisationsgrad für die Sorte Respect in den Stängeln im 2HVJ (2007) in allen Ernteterminen.

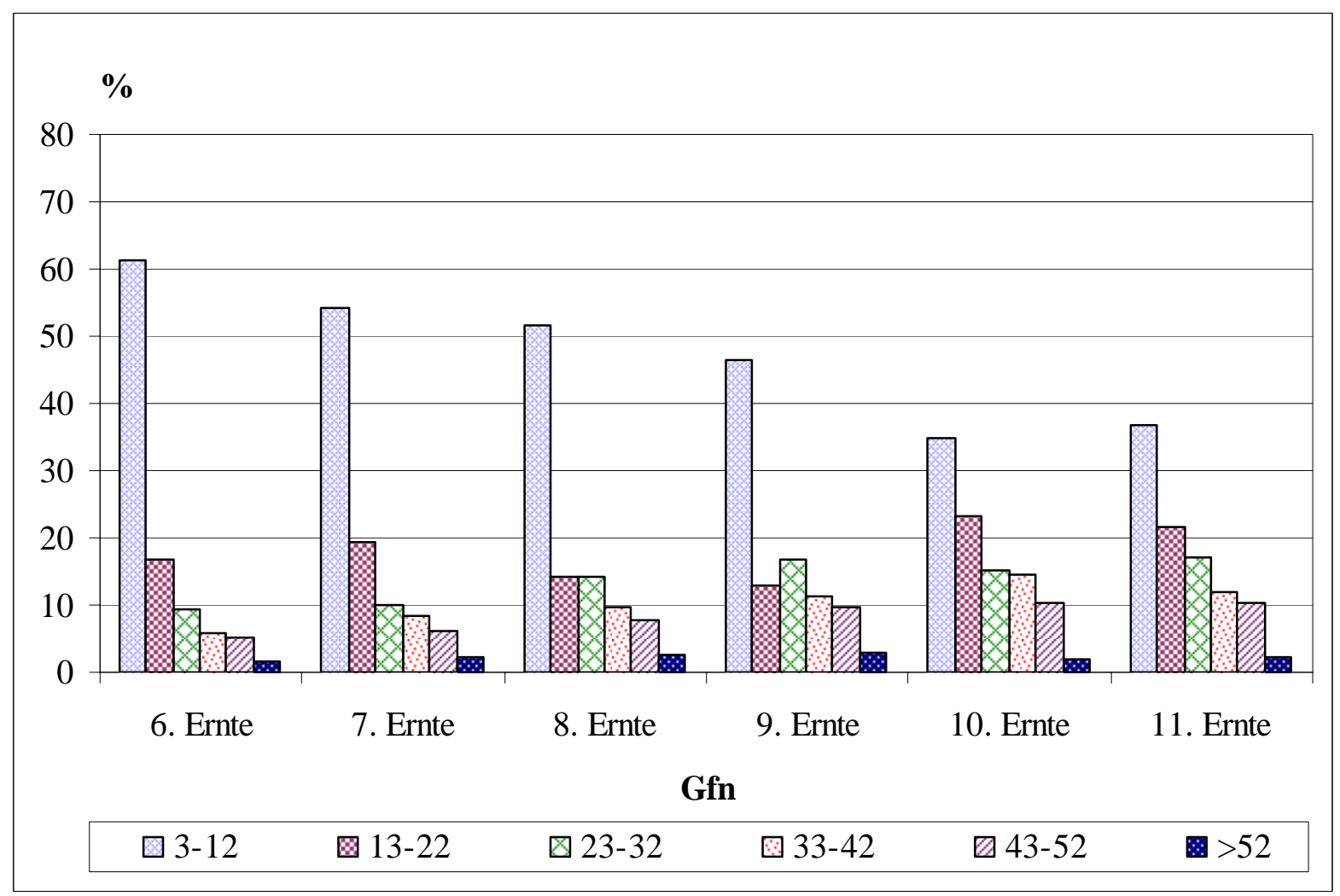

Abb. 52: Verteilung des Fruktangehaltes (\%) nach dem Polymerisationsgrad für die Sorte AberAvon in den Stängeln im 2HVJ (2007) in allen Ernteterminen. 


\section{Diskussion}

\subsection{Sortenversuch}

\subsubsection{Trockenmasseertrag}

Der TM-Ertrag wies keine signifikanten Unterschiede zwischen den Hoch-ZuckerGräsersorten (diploiden Sorten) und tetraploiden Sorten in denselben Reifegruppen der Deutschen Weidelgrassorten auf. Auch gab es keine signifikanten Unterschiede zwischen den Hoch-Zucker-Gräsersorten und übrigen diploiden Sorten der Deutschen Wedelgrassorten in denselben Reifegruppen auf. Nach Untersuchunungen von Johnson et al. (2008) weisen Hoch-Zucker-Gräser nicht unbedingt höhere Ertäge als Vergleichssorten auf. In britischen Studien jedoch wies die Hoch-Zucker-Gras (AberAvon) eine verbesserte Qualität mit höheren Erträgen auf (Eckardt 2007).

Im Mittel hatten die diploiden Sorten einen höheren TM-Ertrag als die tetraploiden Sorten.

Die Sorte Lidaglo (Knaulgras) hatte auf beiden Versuchsstandorten und bei beiden Schnittzeitpunktvarianten die höchsten TM-Erträge. Nach der Beschreibenden Sortenliste des Bundessortenamtes (BSA) und der Deutschen Saatveredelung (DSV) hat diese Sorte ein hohes Ertragsniveau. Daher ist auszugehen, dass die Sorte Lidaglo auf den Versuchsstandorten BS und HS und bei dem Frühschnitt und Spätschnitt eignet und nach Berücksichtigung ihrer Inhaltsstoffe mit anderen Futtergräsern gemischt werden kann (z.B als Mischpartner mit Lidacta geeignet für Feldgras- und Grünlandmischungen (nach DSV)).

Der 1. Schnitt lieferte den Hauptbeitrag zum gesamten TM-Ertrag. Der prozentuale Anteil des 1. Schnittes am Jahres-TM-Ertrag betrug bei FSV zwischen $23 \%$ und $54 \%$ und bei SSV zwischen 35 \% und 63 \%. Nach Lange (2003) werden etwa 30-50 \% des Gesamtertrags im ersten Aufwuchs erzielt. Die höheren Anteile des 1. Schnittes begründen sich einerseits in der Aufwuchsdauer (1. Schnitt erfolgte im Wuchsstadium; Ährenschieben) und andererseits aufgrund des geringeren Wachstums im Sommer im Vergleich zum Frühjahr (Ernst und Rieder, 2000). Der 1. Schnitt ist daher der wichtigste Schnitt. Deshalb ist der 1. Schnitttermin im Frühjahr unerlässlich. Dieser Schnitttermin ist aber stets ein Kompromiss zwischen Futterqualität und Ertrag. Ein früherer Schnitttermin des 1. Schnittes im Frühjahr führt zu niedrigem TM-Ertrag. Bei einem späteren Schnitttermin dagegen nimmt der Bruttoertrag deutlich zu. Allerdings steigt mit späterem Schnitt auch der Rohfasergehalt an. Der Rohproteingehalt sinkt deutlich ab. Mit zunehmendem Rohfasergehalt sinkt die Verdaulichkeit des Futters und hierdurch bedingt nimmt auch der Energiegehalt deutlich ab (Meister und 
Lehmann, 1984; Ernst und Rieder, 2000). Dies stimmt mit den Ergebnissen in der vorliegenden Arbeit überein: Die Trockenmasseerträge der SSV waren höher als die Trockenmasseerträge der FSV, weil der Schnitttermin des 1. Schnittes der SSV ca. 14 Tage später erfolgte. Wie oben gesagt, nahmen die TM-Ertrag mit zunehmender Aufwuchsdauer und fortschreitender phänologischer Entwicklung deutlich an. Der optimale Schnitttermin, wie aus Zahlreichen Literaturstellen bekannt (z.B. Ernst und Rieder, 2000; Haas, 2003), liegt zwischen Beginn und Ende des Blütenstandschiebens (schiebenden Ähre bzw. Rispe, BBCH-Code 43 bis 59, abhängig von der Grasart).

Je nach Versuchsstandort unterscheidet sich der TM-Ertrag jedoch deutlich. In der vorliegenden Arbeit war der TM-Ertrag am Versuchsstandort HS höher als am Versuchsstandort BS. Dies führt auf klimatische Bedienungen und auf Standortmerkmalen zurück.

Das Wachstum auf Grünland ist von den jeweiligen Standortfaktoren abhängig. Haupteinflussfaktoren sind Boden und Klima. Sie beeinflussen sich in ihren Auswirkungen auf das Grünland gegenseitig. Der Bodeneinfluss auf die Zusammensetzung des Pflanzenbestandes und auf die Futterproduktion ist verhältnismäßig gering. Die Wasserversorgung spielt für den Pflanzenbestand und für die Futterproduktion eine überragende Rolle (Ernst und Rieder, 2000). In der vorliegenden Arbeit waren die Niederschläge in allen Versuchsjahren im Versuchsstandort HS deutlich höher als in BS. Dies begründet den höheren TM.Ertrag am Versuchsstandort HS im Vergleich zu BS.

\subsubsection{Wasserlösliche Kohlenhydrate}

Die Veränderungen des Gehaltes und der Verteilung von Kohlenhydraten in der Graspflanze rühren ausschließlich aus dem Zusammenwirken von endogenen (genetische Veranlagung) und exogenen Faktoren (Umwelt- und Bewirtschaftungseinflüsse) her (Matthes, 1986).

Die wichtigsten Einflussfaktoren auf die Kohlenhydrate (insbesondere Fuktane) in der Graspflanzen sind Pflanzenart und -sorte, Entwicklungsstadium der Pflanze, Tages- und Jahreszeit, Temperatur, Lichtintensität, Düngestatus und Nutzungsintensität (Matthes, 1986; Dahlhoff, 2003).

Im folgenden Kapitel wird die Frage diskutiert, inwieweit die wasserlöslichen Kohlenhydrate von Schnittzeitpunkt, Standort, Art und Sorten der Futtergräser, Reife- und Ploidegruppe des Deutschen Weidelgrases und der Jahreszeit beeinflusst werden. Auch wird diskutiert, wie der Fruktangehalt sich bei verschiedenen Futtergräsern verändert.

Der Schnittzeitpunkt ist demnach von Bedeutung, er steht jedoch in enger Beziehung zu der Vegetationsperiode, in der sich die Graspflanzen befinden (Dahlhoff, 2003). Von Borstel und 
Gräßler (2003) untersuchten den Einfluss des Schnitttermins auf den Fruktangehalt; der erste Aufwuchs wies Unterschiede zwischen dem frühen und dem späten Schnitt auf.

In der vorliegenden Arbeit wies die FSV einen höheren Gehalt an WlK im Vergleich zur SSV auf. Die Ursache für den höheren WlK-Gehalt in der FSV ist der Abstand von 14 Tagen zwischen FSV und SSV. Wie aus zahlreicher Literatur zu entnehmen (z.B. Hopf, 1982; Huntington und Pollitt, 2002) ist der höchste Gehalt an WlK beim Entwicklungsstadium „Beginn Ähren-/Rispenschieben“ zu erwarten, wo die FSV in der vorliegenden Arbeit durchgeführt wurde. Bei fortschreitender Pflanzenentwicklung bzw. bei Spätschnitt nimmt der Gehalt an WlK dagegen deutlich ab.

Die Niveauunterschiede der WlK-Gehalte deuten auf einen Standorteinfluss hin. Der Versuchsstandort BS wies im Vergleich zu HS höhere WlK auf. Dies ist auf klimatische Einflüsse und auf Standortmerkmale zurückzuführen.

Die verschiedenen Grasarten unterschieden sich in ihren Fruktan- und WlK-Gehalten. Die artspezifischen Unterschiede sind in der Regel ausschließlich auf den Fruktangehalt zurückzuführen (Matthes, 1986). Weidelgräser lagern in der Regel deutlich höhere Mengen an Fruktan ein als andere Gräserarten wie Knaulgras, Wiesenschwingel und Wiesenlieschgras (Lampeter et al., 1973; Meister und Lenmann, 1984; von Borstel und Gräßler, 2003). In der vorliegenden Arbeit enthielt das Deutsche Weidelgras auch die höchsten Gehalte an WlK und Fruktan. Gräßler und von Borstel (2005) untersuchten in einer Studie den Fruktangehalt von früh-, mittel- und spätreifen, di- und tetraploiden Sorten des Deutschen und Welschen Weidelgrases sowie verschiedene andere Gräserarten des Grünlandes. Auch hier wiesen Deutsches und Welsches Weidelgras die höchsten Fruktangehalte auf; im Mittel der Sorten und der Schnitte lagen die untersuchten Weidelgräser im Mittel der Jahre 2002, 2003 und 2004 bei 5,8 \% i.d. TM (früher bzw später Schnitt); Lieschgras, mehrere Schwingelarten, Knaulgras, Wiesenrispe und Gemeine Rispe, Wiesenfuchsschwanz, Honiggras und Quecke enthielten die niedrigsten Fruktangehalte, die im Mittel um 3,4 \% i.d. TM (früher Schnitt) bzw. 3,5 \% i.d. TM (später Schnitt) lagen.

Auch bei den Sorten innerhalb einer Grasart traten Unterschiede im WlK-Gehalt auf.

Sowohl in den eigenen Ergebnissen als auch in der Literatur (Matthes, 1986; Kalzendorf, 2008) war der WlK-Gehalt bei späten Sorten höher als bei frühen Sorten.

Tetraploide Sorten zeichnen sich zudem durch einen geringfügig höheren Zuckergehalt aus als diploide Sorten (Berendonk, 2005; Kalzendorf, 2008). In der vorliegenden Arbeit enthielten die tetraploiden Sorten des Deutschen Weidelgrases quantitativ mehr WlK als diploide Sorten derselben Reifegruppe. 
Die Hoch-Zucker-Gräser (AberAvon, Aberdart und Abersilo) zeigten im Vergleich zu den diploiden Sorten der gleichen Reifegruppe deutlich höhere Gehalte an WlK. Dies ist auch durch zahlreiche Literaturzitate belegt (z.B. Miller et al., 2001; Gilliland et al., 2003; Wilkins et al., 2003; Martens und Greef, 2003; Beimler und Eickmeyer, 2005; Jänicke, 2005). Im Verlauf eines Jahres gibt es Schwankungen in den WlK-Gehalten.

Der höchste Fruktan- und WlK-Gehalt wurde im Frühjahr gemessen, im Verlauf des Sommers nahmen der Fruktan- und der WlK-Gehalt deutlich ab und stiegen im Herbst wieder an. Eine solche saisonale Veränderungen ermittelten auch Hopf (1982), Larrsson und Steen (1984), Mcgrath (1988), Ulvund und Pestalozzi (1990), Schubiger et al. (1998), Hoffman et al. (2001) und Dahlhoff (2003). In diesen Veränderungen spiegeln sich zunächst klimatische Einflüsse wider (Matthes, 1986). Nach Dahlhoff (2003) ist als die Ursache für die jahreszeitlichen Schwankungen im Fruktangehalt bzw. WlK neben der Lichtintensität insbesondere die Temperatur zu nennen, die sich parallel zu den Jahreszeiten ändert. Bei erhöhter Strahlungsintensität im Frühjahr sind die Voraussetzungen für die hohe Photosyntheserate bereits erfüllt, aufgrund vergleichsweise niedriger Temperaturen aber nicht für entsprechendes Wachstum. Folglich müssen WlK-Überschüsse auftreten. Diese Situation bleibt solange erhalten, bis mit allmählich wirksamem Langtagseinfluss und ansteigenden Temperaturen das Streckungswachstum einsetzt (Peacock, 1975; Robson, 1980). Nach Matthes (1986) ist die Photosyntheseaktivität im Frühjahr generell höher als im Sommer. Dies müsste in Zusammenhang mit der im Frühjahr verstärkten Bildung generativer Triebe stehen.

Mit höheren Temperaturen im Sommer erhöht sich andererseits zugleich auch die Dissimilation. Das erklärt wiederum den in dieser Jahreszeit ermittelten geringeren WlK-Gehalt. Im späten Sommer und Herbst ist die Zuwachsintensität vermindert und die Photosyntheseprodukte übersteigen den verminderten Kohlenhydratbedarf für das Wachstum. Dies ist durch den höheren Fruktangehalt und WlK-Gehalt im Herbst begründet.

Der Energiebedarf der Pflanze ist demnach von entscheidender Bedeutung.

Der Fruktangehalt (\% in den WlK) lag im 2HVJ deutlich höher als im 1HVJ bei allen Gräserarten. Ursache dafür sind die klimatischen Bedingungen. Das 2HVJ (2007) war zu nass und hatte erhöhte Niederschläge. 


\subsubsection{Verdaulichkeit}

Eine hohe Grundfutterqualität ist die Voraussetzung für eine hohe Milchleistung. Qualität und Energiegehalt des wirtschaftseigenen Grundfutters sind in hohem Maße vom Erntezeitpunkt, von der Witterung bei der Ernte, dem Ernteverfahren und dem Silierverlauf abhängig (Beimler und Eickmeyer, 2005). Die Verdaulichkeit des Grundfutters ist neben dem Zuckerund Proteingehalt der wichtigste Parameter des Futterwertes.

Das Blatt : Stängel- Verhältnis beeinflusst die Verdaulichkeit. Blätter und Stängel von Gräsern weisen durch ihre unterschiedliche morphologische und chemische Zusammensetzung eine unterschiedliche Verdaulichkeit auf (Wilman und Altimimi, 1982). Blätter enthalten mehr leicht verdauliche Zellinhaltsstoffe und geringere Anteile an Pflanzenzellwänden als Stängel. Somit sind Faser- und Ligningehalt niedriger als in Stängeln. Auch die chemische Struktur der Kohlenhydratpolymere in den verschiedenen Pflanzenteilen weist Unterschiede auf. Im Verlauf des Reifeprozesses verschiebt sich das Blatt : Stängel-Verhältnis zu einem stärkeren Anstieg des Stängelanteils. So bedingt die geringere Verdaulichkeit der Stängel die Verdaulichkeitssenkung mit der Reife (Meak, 2002). Dies stimmen mit den Ergebnissen in der vorliegenden Arbeit überein: die Verdaulichkeit (ELOS) war bei der FSV (65,1 \% i.d. TM) deutlich höher als in der SSV (62,0 \% i.d. TM). Die Ursache dafür ist, dass mit zunehmendem Alter der Futterpflanzen die Verdaulichkeit absinkt (Ernst und Rieder, 2000).

Die verschiedenen Grasarten unterschieden sich in ihren Verdaulichkeiten.

Die Verdaulichkeit nahm in der Reihenfolge Deutsches Weidelgras, Wiesenschwingelgras, Wiesenschweidelgras, Knaulgras und Wiesenlieschgras ab. Dies lag an ihrem Gehalt an WlK.

Die Sorten der späten Reifegruppe wiesen in der vorliegenden Arbeit einen höheren Gehalt an die Verdaulichkeit auf. Gleiches hat Kalzendorf (2008) erwähnt.

Tetraploide Sorten zeichnen sich zudem durch höhere Verdaulichkeit im Vergleich zu diploiden Sorten derselben Reifegruppe aus. Dies ist vor allem auf dem höheren Gehalt an WlK von den tetraploiden Sorten zurückzuführen.

Die Hoch-Zucker-Gräser (AberAvon und Aberdart) zeigten im Vergleich zu den diploiden Sorten derselben Reifegruppe deutlich höhere Verdaulichkeiten. Dies wird durch den höheren Gehalt an WlK erklärt. In der Literatur wird ebenfalls erwähnt, dass die Hoch-Zucker-Gräser durch ihren deutlich überdurchschnittlichen Zuckergehalt eine bessere Verdaulichkeit zeigten (Matthes, 1986; Anonym, 2007; Eckardt, 2007).

Im Verlauf der Vegetationsperiode bei der FSV und der SSV war die Verdaulichkeit im 1. Schnitt am niedrigsten danach stieg sie in den folgenden Schnitten bei der FSV leicht bzw. bei 
der SSV stark an. Der höhere Fasergehalt im 1. Schnitt, insbesondere bei der SSV, ist als Ursache für die niedrigen Verdaulichkeitswerte wahrscheinlich. Die folgenden Schnitte waren blattreicher deshalb war die Verdaulichkeit hoch.

\subsubsection{Rohproteingehalt}

Der Rohproteingehalt (RP) lag im 1HVJ bei der FSV und SSV über dem Rohproteingehalt des 2HVJ. Der RP lag im 1. Schnitt der FSV und der SSV am Versuchsstandort HS über dem RP des Versuchsstandorts BS. Dies ist auf klimatische Einflüsse und auf Standortmerkmale zurückzuführen.

Der Schnittzeitpunkt ist demnach für den Rohproteingehalt von Bedeutung. Nach Meister und Lehmann (1984) sinkt der Gehalt an Rohprotein, jener an Rohfaser steigt kontinuierlich mit zunehmendem Alter der Pflanzen. In der vorliegenden Arbeit war der RP im 1. Schnitt bei der FSV höher als bei der SSV.

Die geprüften Futtergräser unterschieden sich voneinander. Bei der FSV wies das Wiesenschwingelgras bzw. das Wiesenschweidelgras bei der SSV den höchsten RP auf, d.h. höher als Deutsches Weidelgras. Nach Meister und Lehmann (1984) enthalten auch die Raigräser (Englisch-Raigras (Deutsches Weidelgras), Hybrid- und Italiensch-Raigras) niedrigen Rohproteingehalt als die übrigen Gräser (Wiesenschwingelgras, Timothe und Knaulgras).

Die späten Sorten wiesen einen höheren RP auf als die frühen Sorten. Späte Sorten können nach Matthes (1986) höhere N-Gaben vor allem in den postgenerativen Aufwüchsen besser (d.h. ernährungsphysiologisch günstiger) verwerten als frühe Sorten.

In derselben Reifegruppe wiesen die Sorten Unterschiede auf. In derselben Reifegruppe im 1. Schnitt der FSV wiesen die Sorten Licarta für früh, EGB2 für mittel und EGB4 für spät den höchsten Rohproteingehalt auf. Im 1. Schnitt der SSV wiesen die Sorten EGB1 für früh, EGB2 für mittel und EGB3 für spät den höchsten Rohproteingehalt auf, d.h. höher als HochZucker-Sorten (Abersilo, Aberdart und AberAvon).

Die tetraploiden Sorten des Deutschen Weidelgrases wiesen durch ihre große Blattanlage einen höheren Rohproteingehalt als die diploiden Sorten auf.

Der Rohproteingehalt stieg im Verlauf der Vegetationsperiode vom 1. zum 5. Schnitt an. Dies lag an den blattreichen Gräsern in den Schnitten 2 bis 5. Nach Meak (2002) ist bei den durchschnittlichen Rohproteingehalten ein signifikanter Anstieg von 13,13 \% TS im Mai auf 21,27 \% TS im September zu verzeichnen, zum November sinken die Werte wieder. 


\subsubsection{Rohfasergehalt}

Der Rohfasergehalt (RF) war im 1HVJ bei der FSV und SSV höher als im 2HVJ. Der RF lag im 1. Schnitt der FSV und der SSV am Versuchsstandort HS über dem Wert des Versuchsstandorts BS. Als Ursache hierfür sind klimatische Einflüsse und Standortmerkmale zu nennen.

Der Zusammenhang zwischen dem Pflanzenalter und RF ist allgemein bekannt und wurde von verschiedenen Autoren (Meister und Lehmann, 1984; Wolf und Briemle, 1989; Kittmann und Dyckmans, 1990; Südekum et al., 1990; Taube et al., 1990; Neff, 1991; Spatz und Fricke, 1992; Käding et al., 1993; Hochberg et al., 1994; Ernst und Rieder, 2000) nachgewiesen. Mit zunehmendem Alter der Pflanzen steigt der RF an. In der vorliegenden Arbeit war der RF bei der SSV höher als bei der FSV.

Weiterhin konnte bestätigt werden, dass der RF stark vom Wuchsstadium beeinflusst wird (Sölter, 2007). Die Beobachtung bezüglich des Einflusses des Wuchsstadiums auf den RF machten auch Virkajärvi und Varis (1991) und Nõmmsalu (1994). Bei einer zu späten Nutzung des Bestandes wirkt sich die fortschreitende Lignifizierung und das zunehmende Stängel : Blatt Verhältnis negativ auf den Futterwert aus (Frame, 2005). Aus diesem Grunde kann ein hoher Futterwert nur dann erreicht werden, wenn die Futterpflanze ein bestimmtes Wuchsstadium nicht überschreitet, da sich dieses auf hohe Rohfasergehalte und eine geringere enzymlösliche organische Substanz auswirkt.

Sowohl bei der FSV als auch bei der SSV wies das Knaulgras den höchsten RF auf, das Deutsche Weidelgras enthielt dagegen den niedrigsten RF. Nach Meister und Lehmann (1984) haben die Raigräser (Englisch-Raigras (Deutsches Weidelgras), Hybrid- und Italiensch-Raigras) einen niedrigeren RF als die übrigen Gräser (Wiesenschwingelgras, Timothe und Knaulgras).

In der vorliegenden Arbeit hatten die untersuchten frühen Sorten höheren Rohfasergehalt als die späten Sorten. Auch Kalzendorf (2008) erwähnte, dass die Gräser aus der frühen Reifegruppe den höchsten RF aufwiesen. Späte Sorten wachsen im Frühjahr nicht so schnell wie frühe Sorten, das führt bei gleichem Schnitttermin (wie in der vorliegenden Arbeit) zu einem höheren RF bzw. niedrigen Rohproteingehalt bei den frühen Sorten im Vergleich zu den späten Sorten.

In derselben Reifegruppe im 1. Schnitt der FSV und der SSV wiesen die Sorten Abersilo für früh, Respect für mittel und Sponsor für spät die höchsten RF auf. Dies ist auf Sortenmerkma- 
le zurückzuführen. D. h., dass die Hoch-Zucker-Gräser (mit Ausnahme von Abersilo) einen geringeren RF enthielten.

Sowohl in der vorliegenden Arbeit als auch in der Literatur (Feuerstein und Jerebic, 2007) wiesen die diploiden Sorten des Deutschen Weidelgrases einen höheren RF auf als die tetraploiden Sorten.

Der 1. Schnitt wies einen höheren Rohfasergehalt auf als die folgende Schnitte (2. bis 5. Schnitt). Die Ursache dafür sind blattreiche Gräser im 2. bis 5. Schnitt. Auch Finkler-Schade (1997) stellte in ihrer Studie keine Zunahme sondern einen Rückgang des RF von 22,8 \% TS Ende Mai/Anfang Juni auf 19,9 \% TS Mitte August/Anfang September fest. Auch nach Dahlhoff (2003) wird bei den mittleren Rohfasergehalten der höchste Gehalt von 25,32 \% TS im Mai erzielt, während ein minimaler Gehalt von 17,73 \% TS im November beobachtet wird.

\subsubsection{Die Korrelationen zwischen wasserlöslichen Kohlenhydraten und TM-Ertrag, Verdaulichkeit, Rohproteingehalt und Rohfasergehalt.}

In der vorliegen Arbeit wurde ein negativer Zusammenhang zwischen dem wasserlöslichen Kohlenhydrate-Gehalt und dem TM-Ertrag festgestellt. Je höher der TM-Ertrag ist, desto niedriger waren die WlK.

In der vorliegenden Arbeit wurde ein hoch signifikanter Zusammenhang zwischen dem WlKGehalt und der Verdaulichkeit gefunden, wobei steigende Verdaulichkeit im Gras von steigendem WlK-Gehalt begleitet wurde. Die Verdaulichkeit hängt vom Anteil hochverdaulicher Zucker in der Gesamtpflanze ab. Dieser positive Zusammenhang zwischen dem WlKGehalt und der Verdaulichkeit ist allgemein bekannt und wurde von verschiedenen Autoren (Matthes, 1986; Anonym, 2007; Eckardt, 2007; Feuerstein und Jerebic 2007; Kalzendorf, 2008) nachgewiesen.

Der Rohproteingehalt korreliert negativ mit dem WlK-Gehalt. Der WlK-Gehalt ist umso niedriger, je höher der Rohproteingehalt der Gräser ist. Dies wurde durch die Studie von Matthes (1986) bestätigt. Auch der Zusammenhang zwischen dem Fruktangehalt und dem Rohproteingehalt ist negativ und wurde von zahlreichen Autoren festgestellt (Mackenzie und Wylam, 1957; Hehl und Mengel, 1972; Lang, 1972; Dahlhoff, 2003).

Der Zusammenhang zwischen dem wasserlöslichen Kohlenhydrate-Gehalt und dem Rohfasergehalt war in der vorliegenden Arbeit negativ. Von einem negativen Zusammenhang zwischen dem Fruktangehalt und dem Rohfasergehalt wurde in der Literatur berichtet (Hehl und Mengel, 1972; Kühbauch und Voigtländer, 1975). 


\subsubsection{Schlussfolgerung Sortenversuch}

Die Hoch-Zucker-Gräser wiesen gleiche TM-Erträge wie die tetraploiden Sorten auf. Im Mittel hatten die diploiden Sorten einen höheren TM-Ertrag als die tetraploiden Sorten.

Die Sorte Lidaglo (Knaulgras) hatte die höchsten TM-Erträge.

Der 1. Schnitt lieferte den Hauptbeitrag zum gesamten TM-Ertrag.

Mit zunehmender Aufwuchsdauer und fortschreitender phänologischer Entwicklung, vor allem im ersten Schnitt, nahm der TM-Ertrag zu und die Futterpflanze bildet mehr Rohfaser aus. Der Rohproteingehalt sank deutlich ab. Mit zunehmendem Rohfasergehalt sank die Verdaulichkeit des Futters und hierdurch bedingt nahm auch der Energiegehalt deutlich ab. Aus diesen Zusammenhängen ergibt sich ein Schnittoptimum. Der optimale Schnitttermin, wie in zahlreicher Literatur beschrieben, liegt zwischen Beginn und Ende des Blütenstandschiebens (Schiebende Ähre bzw. Rispe, BBCH-Code 43 bis 59, abhängig von der Grasart). Der TM-Ertrag wurde durch die klimatischen Bedingungen beeinflusst. Die Wasserversorgung spielt für den Pflanzenbestand und für die Futterproduktion eine überragende Rolle.

Der höchste WlK-Gehalt fand sich im früheren Schnitt (FSV) bzw. beim Entwicklungsstadium „Beginn Ähren-/Rispenschieben“. Bei fortschreitender Pflanzenentwicklung bzw. beim späteren Schnitt nahm der Gehalt an WlK deutlich ab.

Das Deutsche Weidelgras wies einen höheren Gehalt an WlK und Fruktan auf als andere Gräserarten, wie z. B. Knaulgras, Wiesenschwingel und Wiesenlieschgras.

Der WlK-Gehalt war bei den späten Sorten höher als bei den frühen Sorten.

Die tetraploiden Sorten des Deutschen Weidelgrases enthielten quantitativ mehr WlK als die diploiden Sorten in derselben Reifegruppe.

Die Hoch-Zucker-Gräser (AberAvon, Aberdart und Abersilo) zeigten im Vergleich zu den diploiden Sorten der gleichen Reifegruppe deutlich höhere Gehalt an WlK.

Im Verlauf der Vegetationsperiode waren der Fruktangehalt und der WlK-Gehalt im Frühjahr (1. Schnitt) am höchsten. Im Verlauf des Sommers nahmen sowohl der Fruktangehalt als auch der WlK-Gehalt deutlich ab; beide stiegen im Herbst wieder an.

Die Verdaulichkeit war beim früheren Schnitt deutlich höher als beim späteren Schnitt.

Die Verdaulichkeit nahm in der Reihenfolge Deutsches Weidelgras, Wiesenschwingelgras, Wiesenschweidelgras, Knaulgras und Wiesenlieschgras ab.

Die Verdaulichkeit war bei den späten Sorten höher als bei den frühen Sorten. 
Tetraploide Sorten zeichnen sich zudem durch eine höhere Verdaulichkeit aus als diploide Sorten in derselben Reifegruppe.

Die Hoch-Zucker-Gräser (AberAvon und Aberdart) zeigten im Vergleich zu den diploiden Sorten derselben Reifegruppe eine deutlich höhere Verdaulichkeit.

Im Verlauf der Vegetationsperiode war die Verdaulichkeit im 1. Schnitt am niedrigsten danach stieg sie in den folgenden Schnitten an.

Der Rohproteingehalt war im 1. Schnitt beim früheren Schnitt deutlich höher als beim späteren Schnitt.

Das Deutsche Weidelgras enthielt einen niedrigen RP als die übrigen Futtergräser (insbesondereim Vergleich zum Wiesenschwingelgras bzw. zum Wiesenschweidelgras).

Die späten Sorten enthielten einen höheren RP als die frühen Sorten.

Die Hoch-Zucker-Gräser (Abersilo, Aberdart und AberAvon) enthielten geringeren RP.

Die tetraploiden Sorten des Deutschen Weidelgrases wiesen einen höheren RP als die diploiden Sorten auf.

Der RP stieg im Verlauf der Vegetationsperiode vom 1. zum 5. Schnitt an.

Der Rohfasergehalt war beim späteren Schnitt deutlich höher als beim früheren Schnitt.

Das Knaulgras wies den höchsten RF, das Deutsche Weidelgras den niedrigsten RF auf.

Die frühen Sorten enthielten einen höheren Rohfasergehalt als die späten Sorten.

Die diploiden Sorten des Deutschen Weidelgrases wiesen einen höheren RF als die tetraploiden Sorten auf.

Die Hoch-Zucker-Gräser (Aberdart und AberAvon) enthielten einen geringeren RF.

Der 1. Schnitt wies einen höheren RF als die folgenden Schnitte (2. bis 5. Schnitt) auf.

Die Zusammenhänge zwischen dem WlK-Gehalt und dem TM-Ertrag, dem RP und dem RF waren negativ. Je höher der TM-Ertrag, der RP und der RF lagen, desto niedriger war der Gehalt an WlK. Der WlK-Gehalt und die Verdaulichkeit befanden sich in einem hoch signifikanten Zusammenhang, wobei steigende Verdaulichkeit im Gras mit steigendem WlKGehalt einhergeht. 


\subsection{Zeitreihenversuch}

\subsubsection{Wasserlösliche Kohlenhydrate und Fruktangehalt}

Die Futterwertbestimmenden Eigenschaften der Futterpflanzen, aber auch die Reservestoffgehalte verändern sich mit dem Entwicklungsstand der Pflanzen. Der Beginn des Ährenschiebens ist nach den Ergebnissen der vorliegenden Arbeit für den WlK-Gehalt und für den Fruktangehalt der Gräser ein markanter Einschnitt: die WlK und der Fruktangehalt nahmen zunächst bis kurz vor Beginn des Ährenschiebens zu, fielen danach aber wieder ab. Zu ähnlichen Ergebnissen kamen auch Kühbauch und Voigtländer (1974), Hopf (1982), Meister und Lehmann (1984), Matthes (1986), Ballard et al. (1990), Schubiger et al. (1998), Huntington und Pollitt (2002). Der Energiebedarf der Pflanze ist demnach von entscheidender Bedeutung (Dahlhoff, 2003). Es ist bekannt, dass mit zunehmender Pflanzenentwicklung, vor allem im ersten Schnitt, WlK-Gehalt absinkt, die Biomasse zunimmt und die Pflanze mehr Rohfaser bildet.

In der vorliegenden Arbeit wies die Hoch-Zucker-Sorte AberAvon die höchsten WlK- und Fruktangehalte auf. Dies wurde in zahlreichen Untersuchungen und von verschieden Autoren ebenfalls (Beimler und Eickmeyer, 2005; Jänicke, 2005; Anonym, 2007) festgestellt.

Hinsichtlich des Gehaltes und der Verteilung der Kohlenhydrate bestehen zwischen den einzelnen Pflanzenorganen Unterschiede. Blätter haben niedrigere Gehalte als Blattscheiden und diese wiederum niedrigere als Stängel (Matthes, 1986). In der vorliegenden Arbeit nahmen die WlK und der Fruktangehalt in der Reihenfolge Stängel, Blätter und Ähren ab. Das Hauptspeicherorgan der Graspflanzen für WlK, und damit auch Fruktan, ist der Stängel (Fulkerson und Donaghy, 2001).

Gehalte an WlK, und damit auch Fruktan, sind je nach Tageszeit unterschiedlich. Die WlK wiesen jedoch in der vorliegenden Arbeit keine signifikanten Unterschiede zwischen den Tageszeiten (8 und 13 Uhr) auf. Dagegen war der Fruktangehalt in der Tageszeit 8 Uhr höher als in der Tageszeit 13 Uhr. Dies widerspricht der Studie von Longland und Cairns (2000), die den niedrigsten Gehalt in den frühen Morgenstunden und den höchsten in den Nachmittags- und frühen Abendstunden fanden. Als Ursache für tageszeitliche Fruktanschwankungen können unterschiedliche Temperaturen, Lichtintensitäten sowie Fruktanreserven vom Vortag angesehen werden (Eagles, 1967a; Longland et al., 1999; Longland und Cairns, 2000). 
Der Einfluss der Temperatur beruht auf ihrer Wirkung auf Wachstum, Photosynthese und Respiration der Pflanze und somit auf ihren Kohlenhydrathaushalt (Matthes, 1986; Fulkerson und Donahgy, 2001). Die Photosyntheseaktivität steigt bei den Arten der gemäßigten Klimazone nur bis zu einem artspezifischen Optimalbereich an (bei Deutschem Weidelgras zwischen 15 und $20^{\circ} \mathrm{C}$ ) und stagniert dann mit zunehmender Temperatur. Im Gegensatz dazu nimmt die Respiration mit steigender Temperatur exponentiell zu (de Vries et al., 1979, Robson, 1981).

Der in der Literatur beschriebene negative Zusammenhag zwischen dem WlK-Gehalt und der Temperatur (Jelmini und Nösberger, 1978a; Voigtländer und Jacob, 1987; Mcgrath, 1988; Baron, 1994; Schubiger et al., 1998; Dahlhoff, 2003) wurde in der vorliegenden Arbeit nicht gefunden. Nach Beobachtungen von Vervuert et al. (2004) sind niedrige Temperaturen mit höheren Fruktangehalten verbunden. Sie erwähnten auch, dass die Beziehung zwischen der Temperatur und der Fruktankonzentration im Gras nicht so eng ist. Eine sichere Prognose der Höhe der zu erwartenden Fruktangehalte in Abhängigkeit von der Temperatur kann nicht getroffen werden, da zahlreiche andere Faktoren die Fruktangehalte maßgeblich beeinflussen. So ist die Temperaturwirkung auf den Fruktangehalt von der Pflanzenart und der Entwicklungsperiode abhängig (Jelmini und Nösberger, 1978a).

Der Gehalt und die Verteilung der Kohlenhydrate werden im Stoffwechsel der Pflanze maßgeblich vom Nährstoffangebot, insbesondere Stickstoff $(\mathrm{N})$ und Kalium (K) beeinflusst (Hehl und Mengel, 1972).

In der vorliegenden Arbeit führte im Verlauf des 1. Aufwuchses des 1HVJ (2006) die höhere N-Düngungsstufe im Mittel der Sorten und der Ernten zum absinkenden Gehalt an Fruktan und an WlK. Zu ähnlichen Ergebnissen kamen auch Auda et al. (1966), Smith (1968), Hehl und Mengel (1972), Lampeter et al. (1973), Matthes (1986), Vervuert et al. (2004).

Steigende Stickstoffgaben führen zur Vergrößerung der Blattfläche und höheren Chlorophyllgehalten, was wiederum die Photosynthese begünstigt (Murata, 1969). Dies führt zu einem verstärkten WlK-Bedarf für die Proteinsynthese sowie für das Wachstum und die Triebbildung, was wiederum einen Abfall der WlK-Gehalte zur Folge hat (Hehl und Mengel, 1972). 


\subsubsection{Verteilung der Fruktangehaltes nach dem Polymerisationsgrad}

Den Fruktanen werden vielfältige Funktionen zugeordnet. Besonders als Schutz bei verschiedensten Stressbedingungen werden Fruktane in spezifischer Rolle aufgeführt. Eine ausführliche Darstellung ist in der Arbeit von Muschick (1998) gegeben. Fruktane als Speicherkohlenhydrate sind vielfältig beschrieben worden (Pavis et al., 2001; Pollock and Cairns, 1991). In Gräsern scheinen aufgrund einer mangelnden oder evolutionistisch ausgebliebenen Stärkesynthese die Fruktane als Speichermedien aufgebaut zu werden (Cairns, 2003; Cairns et al, 2002). In Gräsern variiert die Kettenlänge von Fruktanen. Während beispielsweise in Knaulgras oder Wiesenlieschgras höher-molekulare Fruktane bis zu einem Polymerisationsgrad von bis zu 200 auftreten, finden sich in Weidelgräsern niedermolekulare Oligo-Fruktane bis zu einem Polymerisationsgrad von 60 (Longland und Cairns, 2000).

In dem Versuchsansatz der ,Zeitreihe“ lag das Augenmerk für die Analyse der Fruktane auf ihrer Rolle im Kohlenhydratspeicher zu untersuchen. Die Funktion der Fruktane besteht darin, einen Zwischenspeicher für die Assimilate zu bilden. Über die Blätter werden die Assimilate gebildet, die als Fruktane in Blatt und Stängel zwischengespeichert werden. Besonders der Stängel wird in dieser Funktion als ein temporärer Pool angesehen. Aus diesem Zwischenspeicher kann der Kohlenhydratbedarf besonders während der Kornfüllung gespeist werden. Auf diesen Zwischenspeicher wird umso mehr zurückgegriffen, je weniger aus der aktuellen Assimilation über das Blatt nicht nachgeliefert werden kann. Diese Situation tritt z.B. unter abiotischem Stress oder unter Krankheitsbefall ein (Armiard et al., 2003; Morcuende et al., 2004). Die Fruktane sind wie auch die Stärke physiologisch inaktiv, womit sie sich als Speicherkohlenhydrate eignen. Mit zunehmendem Entwicklungsablauf werden grundsätzlich höherkettige Polymerisationsgrade aufgebaut.

Die untersuchten Sorten aus dem ,Zeitreihenversuch' unterschieden sich eindeutig in ihrer Dynamik, spezifische Fruktanmuster auszubilden. Dieses war besonders in den Blättern und Stängeln deutlich. Zunächst verhielten sich alle Sorten und besonders die beiden divergierenden Sorten Respect und AberAvon so, dass im Entwicklungsverlauf der Polymerisationsgrad von kurz- zu langkettigen Fraktionen aufgebaut wurde. Der wesentliche Unterschied zwischen beiden Sorten scheint darin zu liegen, dass dieser Prozess bei der Sorte Respect nach diesem vorgegebenen Muster abläuft. Bei dem Hochzuckergras AberAvon deutet sich aber an, dass der kurzkettige Polymerisationsgrad verstärkt ausgebildet wird und die nachfolgenden Fraktionen nicht in dem Maße aufgebaut werden, wie es bei der Sorte Respect der Fall ist. Damit steht diese 1. Fraktion vermutlich in einem direkteren bzw. unmittelbareren Austausch 
mit dem Assimilatpool der metabolischen Kohlenhydrate. Möglicherweise kann dieser Pool direkter gespeist werden, was zu den erhöhten Gehalten an WlK führen kann. Untersuchungen und Ausführungen von Cairns (2003) deuten in eine ähnliche Richtung der Frukatnbiosynthese in Gräsermaterial.

\subsubsection{Schlussfolgerung Zeitreihenversuch}

Die Gehalte an WlK und Fruktan nahmen zunächst bis kurz vor Beginn des Ährenschiebens zu, fielen danach aber wieder ab.

Die Sorte AberAvon wies die höchsten WlK- und Fruktangehalte auf.

Die WlK- und Fruktangehalte nahmen in der Reihenfolge Stängel, Blätter und Ähren ab.

Die Gehalte an WlK, und damit auch Fruktan, veränderten sich in den Tageszeiten.

Der in der Literatur beschriebene negative Zusammenhag zwischen WlK-Gehalt und Temperatur wurde in der vorliegenden Arbeit nicht gefunden.

Mit Erhöhung der N-Düngung nimmt der Gehalt an WlK und Fruktan ab.

Im Zeitreihenversuch zeigte sich, dass die Hoch-Zucker-Sorte AberAvon, im Gegensatz zu den anderen untersuchten Sorten, verstärkt kurzkettige Polymere bildet. Die langkettiges Fruktanen wurden in diese Sorte in geringerem Maße aufgebaut, als in den übrigen Sorten beobachtet. Diese sortenspezifischen Unterschied in der Kohlenhydrat-Biosynthese kann als Ursache für den hohen Gehalt an WlK in der Sorte AberAvon interpretiert worden.

\subsection{Fazit}

Aus den durchgeführten Versuchen kann abgeleitet werden, dass der Zusammenhang zwischen dem WlK-Gehalt und der Verdaulichkeit positiv war. Die späten Sorten, die tetraploiden Sorten und Hoch-Zucker-Sorten wiesen höheren WlK-Gehalte auf. Dies könnte die Verdaulichkeit der Futtergräser verbessern, wenn sie richtig eingesetzt werden.

Der optimale Schnittzeitpunkt der verwendeten Futtergräser sollte unbedingt beachtet werden. Beim früheren Schnittzeitpunkt bzw. beim Entwicklungsstadium „Beginn Ähren/Rispenschieben“ wiesen die Futtergräser einen höheren WlK-Gehalt, eine höhere Verdaulichkeit, einen höheren Rohproteingehalt und einen niedrigeren Rohfasergehalt auf als bei fortschreitender Pflanzenentwicklung bzw. beim späteren Schnittzeitpunkt. 


\section{Zusammenfassung}

Ziel der vorliegenden Arbeit war es, experimentell zu ermitteln inwieweit Hoch-ZuckerGräsersorten die Verdaulichkeit und die Grundfutterqualität durch Verschiebungen der wasserlöslichen Kohlenhydrate beeinflussen.

Die Untersuchungen in der vorliegenden Arbeit beinhalteten zwei Versuche: Ein Sortenversuch mit 20 Sorten verschiedener Futtergräserarten und ein Zeitreihenversuch mit fünf Sorten des Deutsches Weidelgrases. Der Sortenversuch wurde im Frühjahr 2005 als Feldanlage mit zwei Schnitterminen (Früh- und Spätschnitt) auf zwei Standorten (Braunschweig (BS) und Hof Steimke (HS)) angelegt und beinhaltete zwei Hauptversuchsjahre von 2006 bis 2007 mit jeweils fünf Schnitten in BS bzw. vier in HS. Die Trockenmasseerträge der jeweiligen Schnitte wurden ermittelt. Der Schnitttermin des 1. Schnittes der Frühschnittvariante richtete sich nach dem Reifegrad der Sorte Respect (Beginn des Ähren- bzw. Rispenschiebens). Der Schnitttermin des 1. Schnittes der Spätschnittvariante erfolgte ca. 14 Tage später. Die Folgeschnitte wurden im vier Wochen Rhythmus beerntet.

Anhand der gewonnenen Grasproben wurden folgende Parameter analysiert: Gesamtzuckerund Fruktangehalt, organische Substanz, Rohprotein- und Rohfasergehalt.

Der Zeitreihenversuch wurde im Herbst 2005 am Standort BS mit zwei N-Düngungsvarianten angelegt und beinhaltete zwei Hauptversuchsjahre von 2006 bis 2007. Die erste Beerntung fand drei Wochen nach Vegetationsbeginn statt. Im Versuchsjahr 2006 wurden einmal wöchentlich zwei Ernten jeweils morgens und mittags durchgeführt. Dies erfolgte bis zum Ende der Blüte, insgesamt wurden vier Ernten durchgeführt. Im Versuchsjahr 2007 erfolgte die Beerntung zweimal wöchentlich gegen mittags. Dies erfolgte bis zum Ende der Blüte plus 2 Ernten mehr, insgesamt wurden 11 Ernten durchgeführt.

Bei jeder Ernte wurden die Phänologischen Stadien (BBCH) bestimmt. Aus Teilproben wurde Gesamttrockenmasse ermittelt. Anschließend wurde in Blatt und Stängel getrennt. Es wurden die WlK- und Fruktan-Gehalte der Gesamtprobe und der in Stängel, Blatt und Ähre (Versuchsjahr 2007), separierten Proben mit HPLC bestimmt. Es wurde zusätzlich der Polymerisationsgrad des Fruktans mit HPLC bestimmt. 


\section{Folgende Ergebnisse wurden erzielt:}

\section{Sortenversuch}

- Die Hoch-Zucker-Gräser wiesen gleiche TM-Erträge wie die tetraploiden Sorten auf.

- Im Mittel hatten die diploiden Sorten einen höheren TM-Ertrag als die tetraploiden Sorten.

- Die Sorte Lidaglo (Knaulgras) hatte die höchsten TM-Erträge.

- Der 1. Schnitt lieferte den Hauptbeitrag zum gesamten TM-Ertrag.

- Der optimale Schnitttermin liegt zwischen dem Beginn und dem Ende des Blütenstandschiebens (Schiebenden Ähre bzw. Rispe, BBCH-Code 43 bis 59, abhängig von Grasart).

- Der TM-Ertrag wurde von klimatischen Bedingungen beeinflusst; die Wasserversorgung spielte für den Pflanzenbestand und für die Futterproduktion eine überragende Rolle.

- Der frühe Schnitt (FSV) bzw. zum Entwicklungsstadium „Beginn Ähren-/Rispenschieben“ wies einen höheren WlK-Gehalt, eine höhere Verdaulichkeit, einen höheren Rohproteingehalt und einen niedrigeren Rohfasergehalt auf als bei fortschreitender Pflanzenentwicklung bzw. bei späterem Schnitt.

- Das Deutsche Weidelgras wies einen höheren Gehalt an WIK und Fruktan, eine höhere Verdaulichkeit, einen niedrigeren Rohprotein- und Rohfasergehalt als andere Gräserarten wie Knaulgras, Wiesenschwingel und Wiesenlieschgras auf.

- Die späten Sorten des Deutschen Weidelgrases enthielten einen höheren WlK-Gehalt, eine höhere Verdaulichkeit, einen höheren Rohproteingehalt und einen niedrigeren Rohfasergehalt als die frühen Sorten.

- Die tetraploiden Sorten des Deutschen Weidelgrases wiesen einen höheren WlK-Gehalt, eine höhere Verdaulichkeit, einen höheren Rohproteingehalt und einen niedrigeren Rohfasergehalt als die diploiden Sorten auf.

- Die Hoch-Zucker-Gräser zeigten im Vergleich zu den diploiden Sorten derselben Reifegruppe einen deutlich höheren WlK-Gehalt, eine höhere Verdaulichkeit und geringeren Rohprotein- und Rohfasergehalt.

- Im Verlauf der Vegetationsperiode wies der 1. Schnitt einen höheren WlK-Gehalt, eine niedrigere Verdaulichkeit, einen niedrigeren Rohproteingehalt und einen höheren Rohfasergehalt als die folgenden Schnitte (2. bis 5. Schnitt) auf.

- Die Zusammenhänge zwischen dem WlK-Gehalt und dem TM-Ertrag, dem Rohproteingehalt und dem Rohfasergehalt waren negativ korreliert und zwischen dem WlK-Gehalt und der Verdaulichkeit positiv korreliert. 


\section{Zeitreihenversuch}

-Die höchsten WlK- und Fruktangehalte wurden im Entwicklungsstadium „Beginn Ähren/Rispenschieben“ gemessen; sie fielen danach aber wieder ab.

- Die Sorte AberAvon wies die höchsten WlK- und Fruktangehalte auf.

- Die WlK- und Fruktangehalte nahmen in der Reihenfolge Stängel, Blätter und Ähren ab.

- Die Gehalte an WlK, und damit auch Fruktan, veränderten sich in den Tageszeiten.

- Mit Erhöhung der N-Düngung nahm der Gehalt an WlK und Fruktan ab.

- Die Hoch-Zucker-Sorte AberAvon bildete im Gegensatz zu den anderen untersuchten Sorten, kurzkettige Polymere aus. Die langkettiges Fruktane wurden in dieser Sorte in geringerem Maße aufgebaut, als es in den übrigen Sorten beobachtet wurde. 


\section{Summary}

\section{Investigations on water soluble carbohydrates, yield and ingredients in forage grasses for improvement of digestibility}

The aim of this study was to investigate experimentally to what extent high-sugar grass varieties influence the digestibility and feed quality by changes in water soluble carbohydrates.

The appraisal in this study consists of two experiments: A variety trial using 20 different forage grass species and a time series experiment comparing five varieties of perennial ryegrass. The variety trial was established in spring 2005 as plot design with two harvest dates (early and late) at two sites, Braunschweig (BS), and Hof Steimke (HS) in two experimental years 2006 to 2007 having in total 5 harvests at BS and 4 in HS, respectively. Dry matter yield of the individual harvests was analyzed. The date of the first harvest in the early harvest variant was set in accordance to the stage of maturity of the variety "Respect" (beginning of spike or panicle elongation). The first harvest date of the late harvest variant was chosen 14 days later. The following harvests were carried out in 4 weeks intervals.

From these grass samples the following parameters were analyzed: total sugar and fructan content, organic matter, crude protein, and crude fiber content.

The time series experiment was established in autumn 2005 at the site BS comparing two Nfertilizer variants in the experimental years 2006 and 2007. The first harvest was carried out three weeks after begin of vegetation. In 2006, once in a week two harvests were done, in each case one in the morning and one at noon time. This was continued until end of flowering with four harvests in total. In 2007, harvest was carried out twice a week at noon time with 11 harvests in total ending two harvest dates after the stage "end of flowering".

At each harvest the phenological stage $(\mathrm{BBCH})$ was determined. Parts of the samples were used for dry matter analysis. Samples were separated in leaves and stems. The water soluble carbohydrates and fructan content was determined by HPLC in the total sample as well as separately in leaves, stems, and spikes (2007). Additionally, the degree of polymerization in the fructan fraction was analyzed. 
The following results were achieved:

\section{Variety experiment}

- The high-sugar grasses showed the same dry matter yields like the tetraploid varieties.

- In a mean, diploid varieties exceeded the dry matter yield of tetraploid varieties.

- The variety Lidalgo (orchard grass) achieved the highest dry matter yields.

- $\quad$ The first harvest had the biggest contribution to the total dry matter yield.

- The optimal harvest date is between begin and end of inflorescence elongation (spike or panicle elongation, BBCH-code 43 to 59, depending on the grass species).

- Dry matter yield was influenced by climatic conditions; water supply played a significant role for plant establishment and fodder production.

- The early harvest and accordingly the harvest at developmental stage "begin of spike/panicle elongation" showed a higher content of water soluble carbohydrates, a better digestibility, a higher crude protein content, and a lower crude fiber content than at later harvests, respectively.

- Perennial ryegrass exhibited a higher water soluble carbohydrates and fructan content, a better digestibility, and a lower crude protein and crude fiber content in comparison to other grass species like orchard grass, meadow fescue, and timothy.

- The late varieties of perennial ryegrass showed a higher content of water soluble carbohydrates, a better digestibility, a higher crude protein content, and a lower crude fiber content than early varieties.

- Tetraploid perennial ryegrass varieties exhibited a higher content of water soluble carbohydrates, a better digestibility, a higher crude protein content, and lower crude fiber content than diploid varieties.

- The high-sugar grasses showed a significant higher content of water soluble carbohydrates, a better digestibility, and a lower crude protein and crude fiber content in comparison to diploid varieties.

- During vegetation period, the first harvest exhibited a higher content of water soluble carbohydrates, a worse digestibility, a lower crude protein content, and higher crude fiber content than the following harvests (2. to 5. harvest).

- Content of water soluble carbohydrates and dry matter yield as well as crude protein content and crude fiber content were negatively, whereas the content of water soluble carbohydrates and digestibility were positively correlated. 


\section{Time series experiment}

- The highest contents of water soluble carbohydrates and fructan were found at the developmental stage of "begin of spike/panicle elongation"; later on, these parameters decreased.

- The variety AberAvon showed the highest contents of water soluble carbohydrates and fructan.

- Contents of water soluble carbohydrates and fructan decreased in the order stems, leaves, and spikes.

- The contents of water soluble carbohydrates and therefore also fructan change with time of the day.

- By increasing $\mathrm{N}$ fertilizer amount the contents of water soluble carbohydrates and fructan decreased.

- The high sugar variety AberAvon contained short chain fructans in contrast to the other investigated varieties. Long chain fructans were produced to a lower extent in this variety than in the other varieties. 


\section{$7 \quad$ Literaturverzeichnis}

Alberda, T., (1960): The effect of nitrate nutrition on carbohydrate content of Lolium perenne. Proc. 8th Int. Grassld. Congr. Reading, 612-615.

Alberda, T., (1965): The influence of temperature, light intensity and nitrat concentration on dry-matter production and chemical composition of Lolium perenne (L.). Neth. J. Agric. Sci., 13, Nr. 4, 335-360.

Amiard, V., Morvan-Bertrand, A., Billard, JP., Huault, C., Keller, F. und Prud'homme, MP., (2003): Fructans, but not the sucrosyl-galactosides, raffinose and loliose, are affected by drought stress in perennial ryegrass. Plant Physiol. 2003 Aug; 132(4):2218-29.

Anonym, (2007): Futterqualität ist mehr als nur Zucker.

http://www.dsv-saaten.de/data/pdf/13/01/00/Country-Futterqualitaet.pdf (13.05.2008).

Auda, H., Blaser, R.E. und Brown, R.H., (1966): Tillering and carbohydrate contents of orchardgrass as influenced by environmental factors. Crop. Sci. 6, 139-143.

Bailey, R.W., (1973): Structural carbohydrates. In: Chemistry and Biochemistry of Herbage. Vol. 1 (Eds. G.W. Butler and R.W. Bailey), Academic Press, London und New York, 157211.

Ballard, R. A., Simpson, R. J. und Pearce, G. R., (1990): Losses of the digestible components of annual ryegrass (Lolium rigidum Gaudin) during senescence.

Aust. J. Agric. Res., 41, 719-731.

Baron, J. P., (1994): Einfluß der Wachstumstemperatur auf die Entwicklung des Mahlwiderstandes von Lolium multiflorum Lam.

Das Wirtschaftseigene Futter, 40, 74-89.

Beimler, M. und Eickmeyer, F., (2005): Hoch Zucker Gräser - Eine neue Sortengeneration für höchste Ansprüche an die Futterqualität. Mitteilungen der Arbeitsgemeinschaft Grünland und Futterbau 2005, Band 7. 49 Jahrestagung, 25. bis 27. August 2005 in Bad Elster, 107-110. 
Berendonk, C., (2005): Anbau von Gras und Kleegras im Herbst.

Landwirtschaftskammer Nordrhein-Westfalen, http://www.landwirtschaftskammer-nrw.de/fachangebot/ackerbau/gruenland/kleegrasherbst.htm (11.05.2008).

Budras, K.-D., Scheibe, K., Patan, B., Streich, W. J., und Kim, K., (2001): Laminitis in Przewalski horses kept in a semireserve. J. Vet. Sci., 2, Nr. 1, 1-7.

Cairns A. J., (2003): Fructan biosynthesis in transgenic plants.

Journal of Experimental Botany, Volume 54, Special Issue 382, 3 January 2003, pp. 549-567 (19).

Cairns, A. J., Cookson, A., Thomas, B. J. und Turner, L. B., (2002): Starch metabolism in the fructan-grasses: Patterns of starch accumulation in excised leaves of Lolium temulentum (L.). J. Plant Physiol., 159, 293-305.

Chartterton, N. J., Thornley, W. R., Harrison, P. A. und Bennett, H., (1991): DP-3 and DP-4 oligosaccharides in temperate and tropical grass foliage grown under cool temperatures. Plant Physiol. Biochem., 29, Nr. 4, 367-372.

Coenen, M., Vervuert, I., (2004): Fructangehalt im Weidegras und Hufrehe bei Pferden. Tagungsband. 45. Fachtagung des DLG-Ausschusses „gräser, Klee und Zwischenfrüchte“, Vorträge der Fachtagung vom 30. November und 1. Dezember 2004 in Fulda, 110-114.

Daccord, R., (1992): Grenzen der Milchleistung. Landfreund 1992, 52, 11-13.

Dahlhoff, S., (2003): Fruktangehalt im Gras von Pferdeweiden während der Weidesaison 2002. Dissertation Tierärztliche Hochschule Hannover.

de Vries, F.W.T.P., Witlage, J.M. and Kremer, D., (1979): Rates of respiration and of increase in structural dry matter in young wheat, ryegrass and maize plants in relation to temperature, to water stress and to their sugar content. Ann. Bot. 44, 595-609. 
Deinum, B., (1966b): Influence of some climatological factors on chemical composition and feeding value of herbage. Proc. Xth Int. Grassl. Congr., Helsinki 1966, Section 2, Paper 15, 415-418.

Donaghy, D. J. und Fulkerson, W. J., (1998): Priority for allocation of water-soluble carbohydrate reserves during regrowth of Lolium perenne.

Grass and Forage Sci., 53, 211-218.

Eagles, C. F., (1967a): Diurnal variation of carbohydrates in cocksfoot (Dactylis glomerata). J. Sci. Food Agric., 18, 186-187.

Eckardt, T., (2007): Mehr Zucker im Gras.

BLW 10, 2007, http://www.saatzucht-steinach.de/presse/zucker2007.pdf (13.05.2008).

Eickmeyer, F., (2004): Hoch-Zucker-Gräser - Hintergrund, Entstehung und erste Ergebnisse deutscher Prüfansteller. Tagungsband. 45. Fachtagung des DLG-Ausschusses „gräser, Klee und Zwischenfrüchte“, Vorträge der Fachtagung vom 30. November und 1. Dezember 2004 in Fulda, 19-28.

Ernst, P. und Rieder, J., (2000): Dauergrünland. In: Lütke Entrup, N., J. Oehmichen (Hrsg) (2000): Lehrbuch des Pflanzenbaues: Bd. 2, Kulturpflanzen. Verlag Th. Mann, Gelsenkirchen, 692 - 754 .

Feuerstein, U. und Jerebic, W., (2007): An der Qualitätsschraube drehen! Gräserzüchter arbeiten mit Erfolg an der Verbesserung der Futterqualität. Innovation - DSV-Magazin 1/2007 S. 4-6.

Finkler-Schade, C., (1997): Felduntersuchung während der Weideperiode zur Ernährung von Fohlenstuten und Saugfohlen sowie zum Wachstumsverlauf der Fohlen. Bonn, Rheinische Friedrich-Wilhelms-Universität, Dissertation.

Frame J., (2005): Forage legume profiles. Forage Legumes for Temperate Grasslands. FAO; Italy and Enfield, NH, USA, Science Publishers Incorporation. 
Fulkerson, W. J., (1994): Effect of redefoliation on the regrowth and water soluble carbohydrate content of Loliumperenne. Aust. J. Agric. Res., 45, 1809-1815.

Fulkerson, W. J. und Donaghy, D. J., (2001): Plant-soluble carbohydrate reserves and senescence - key criteria for developing an effective grazing management system for ryegrass-based pastures: a review. Austr. J. Exp. Agric., 41, 261-275.

Gatford, K. L., Simpson, R. J., Siever-Kelly, C., Leury, B. J. und Dove, H., (1999): Spraytopping annual grass pasture with glyphosate to delay loss of feeding value during summer. I. Effects on pasture yield and nutritive value.

Aust. J. Agric. Res., 50, 453-464.

Gilliland, T.J., Barrett, P.D., Agnew, R.E., Fearon, A.M. und Wilson, F.E.A., (2003): Variation in herbage quality and grazing value traits in perennial ryegrass (Lolium perenne L.) varieties. Vortr. Pflanzenzüchtung, 59, 11-19.

Gräßler, J. und von Brostel, U., (2005): Bestimmung der Fruktangehalte verschiedener Gräserarten. Landwirtschaftskammer Hannover, Versuchsbericht Grünland 2002, 2003, 2004.

Greef, J.M., Pahlow, G. und Martens D. S., (2005): Eu-Gemeinschaftsprojekt SweetGrass: Silierung energiereicher Gräser für die nachhaltige Produktion in der Wiederkäuerernährung. mitteilungen der landwirtschftskammer aktuelles aus grünland und futterbau 578/2005: 7273, Kiel: Landwirtschaftskammer Schleswig-Holstein, 2005.

Gruber, L., Steinwender, R., Baumgarten, W., Schauer, A., Häusler, H., Guggenberger, Th., Uray, G., Wenzl, W., Steiner, B., Sobotik, M. und Krimberger, K., (1995): Einfuß von Grundfutterqualität und Kraftfutter auf Leistung, Stoffwechsel und Wirtschaftlichkeit von Kühen der Rasse Fleckvieh und Holstein Frisian. Bericht über die 22. Tierzuchttagung „Aktuelle Forschungsergebnisse und Versorgungsempfehlungen in der Rindermast und Milchviehfütterung“, BAL Gumpenstein, 9.-10. Mai 1995 1-49. 
Haas, G., (2003): Rotkleegras: Arten- und Sortenwahl der Gräser [Grass - red clover mixture: Choice of grass species and varieties], in Dokumentation 10 Jahre Leitbetriebe Ökologischer Landbau in Nordrhein-Westfalen.. Schriftenreihe des Lehr- und Forschungsschwerpunktes „Umweltverträgliche und Standortgerechte Landwirtschaft“ Nr. 105, Seite(n) pp. 149-158. Landwirtschaftliche Fakultät der Rheinischen Friedrich-Wilhelms-Universität Bonn.

Hasselmann, L., Fischer, A., Schalitz, G., (2003): Untersuchungen zur Entwicklung von Stoffgehalten im Aufwuchs von Niedermoorstandweiden in Abhängigkeit vom Vegetationsverlauf. - Archives of Agronomy and Soil Science.49 (6): 613-621.

Hasselmann, L., Fischer, A., Schalitz, G., (2004): Untersuchungen zur Verdaulichkeit und zum Energiegehalt von Futterpflanzen auf Niedermoorstandweiden in Abhängigkeit vom Vegetationsverlauf. - Archives of Agronomy and Soil Science.50 (2): 201-210.

Hehl, G. und Mengel, K., (1972): Der Einfluß einer variierten Kalium- und Stickstoffdüngung auf den Kohlenhydratgehalt verschiedener Futterpflanzen.

Landwirtsch. Forsch., Sonderheft 27, Nr. 2, 117-129.

Hertwig, F., (2004): Energetische Bewertung von Gras und Graskonservaten von Grünlandflächen unterschiedlicher Bewirtschaftungsintensität. . 45. Fachtagung des DLG-Ausschusses „gräser, Klee und Zwischenfrüchte“, Vorträge der Fachtagung vom 30. November und 1. Dezember 2004 in Fulda, 11-18.

Hochberg, H., Matthes, I., Richter, G. und Zopf, D., (1994 ): "Einfluß eines späten Schnittzeitpunktes auf Verdaulichkeit und Energiegehalt des Primäraufwuchses verschiedener Grünlandbestände." VDLUFA Schriftenreihe, 38 , 393-396.

Hoffman, R. M., Wilson, J. A., Kronfeld, D. S., Cooper, W. L., Lawrence, L. A., Sklan, D. und Harris, P. A., (2001): Hydrolyzable carbohydrates in pasture, hay, and horse feeds: Direct assay and seasonal variation. J. Anim. Sci., 79, 500-506.

Hopf, M., (1982): Gehalte, Molekulargewichte und mikrobieller Abbau von Fruktosan aus Dactylis glomerata, Phleum pratense und Lolium perenne.

Diss. Tu München, Freising-Weihenstephan. 
Humphreys, M. O., (1989): Assessment of perennial ryegrass for breeding:

II. Components of winter hardiness; Euphytica, v. 41, S. 99-106.

Huntington, P. und Pollitt, C. C., (2002): Nutrition and the equine foot.

Proc. 2002 equine Nutrition Conf. Kentucky equine Research, Lexington, 149-162.

Jänicke, H., (2005): Hoch-Zucker-Gras auf einem nordostdeutschen Niedermoorstandort erste Ergebnisse. Mitteilungen der Arbeitsgemeinschaft Grünland und Futterbau 2005, Band 7. 49 Jahrestagung, 25. bis 27. August 2005 in Bad Elster, 127-130.

Jelmini, G. und Nösbergger, J., (1978a): Einfluß der Temperatur auf die Ertragsbildung, den Gehalt an nichtstrukturbildenden Kohlenhydraten und Stickstoff von Festuca pratensis Huds., Lolium multiflorum Lam., Trifolium pratense (L.) und Trifolium repens (L.). Z. Acker- und Pflanzenbau, 146, 143-153.

Johnson I. R., Chapman, D. F., Snow, V. O., Eckard, R. J., Parsons, A. J., Lambert, M. G. und Cullen, B. R., (2008): DairyMod and EcoMod: biophysical pasture-simulation models for Australia and New Zealand.

Australian Journal of Experimental Agriculture. 200848 (5). p. 621.

Junk, J. und Fisch, R., (2008): Neue Mischungs- und Sortenempfehlung 2008-2009.

http://agrarinfo.aspdienste.de/Internet/global/themen.nsf/116a0a5933eb78e9c1256fe2003ef15

9/6d066b5789ba151ec12571700032f32b/\$FILE/Mischungs-

\%20und\%20Sortenempfehlung\%202008_2009.pdf (05.2008).

Käding, H. und Kreil, W., (1990): Optimale Nutzung und Bewirtschaftung von

Rohrglanzgrasbeständen. Arch. Acker- u. Pflanzenbau Bodenkd., 34, 489-495.

Käding, H., Schalitz, G. und Leipnttz, W., (1993): Veränderungen der Gehalte an pflanzlichen Inhaltsstoffen durch extensive Bewirtschaftung von Niedermoorgrünland. Das wirtschaftseigene Futter, 39, 157-167.

Kalzendorf, Ch., (2008): Sortenkenntnis für beste Grundfutterqualität wichtig. Innovation - DSV-Magazin 1/2008 S. 4-7. 
Kittmann, U. und Dyckmans, A., (1990): Extensive Grünlandbewirtschaftung - Auswir-kung auf den Futterwert und die Konservierungseignung, 1. Teil:Auswirkung auf den Futterwert. Jahrestagung der Arbeigemeischaft für Grünland und Futterbau in der Gesellschaft für Pflanzenbauwissenschaften, Tagungsbericht 102-109.

Kühbauch, W. und Voigtländer, G., (1974): Vegetationskegelentwicklung und Variabilität von Zuckergehalten im Knaulgras (Dactylis glomerata L.).

Z. Acker- und Pflanzenbau, 140, Nr. 84, 85-99.

Kühbauch, W., (1972a): Veränderungen der Gehalte an Glucose, Fructose, Saccharose und Fructosan sowie des Polymerisationsgrades von Fructosanmolekülen in Blättern und Stengeln einiger Knaulgrassorten während des Wachstums.

Landwirtsch. Forsch., 26, Nr. 2, 173-181.

Kühbauch, W., (1978): Die Nichtstrukturkohlenhydrate von Gräser des gemäßigten Klimabereiches, ihre Variationsmöglichkeiten und mikrobielle Verwertung. Landw. Forsch. 31, 251268.

Lampeter, W., Matthies, H. und Tchaptchet, A., (1973): Untersuchungen über die Verdaulichkeit, den Gehalt an löslichen Kohlenhydraten und Nitratstickstoff der Futtertrockenmasse einiger Gräsereinsaaten in Abhängigkeit von der NDüngung.

Arch. Acker- u. Pflanzenbau u. Bodenkd., 17, Nr. 5, 363-373.

Lang, V., (1972): Lösliche Kohlenhydrate und Spurenelemente im Mähweidefutter in Abhängigkeit von Witterung und Bewirtschaftung.

München, Institut für Grünlandlehre der Technischen Universität, Dissertation.

Lange, G., (2003): Gibt es die optimale Ansaatmischung?

Innovation - DSV-Magazin 1/2003 S. 4-7.

Larrsson, K. und Steen E., (1984): Nitrogen and carbohydrates in grass roots sampled with a mesh bag technique. Swedish J. Agric. Res., 14, 159-164. 
Lenuweit, U., Gharadjedaghi B., (2002): Biologische Basisdaten zu Lolium perenne, Lolium multiflorum, Festuca pratensis und Trifolium repens.

GFN - Gesellschaft für Freilandökologie und Naturschutzplanung mbH, Bayreuth, Im Auftrag des Umweltbundesamtes.

Herausgeber: Umweltbundesamt, Berlin, April 2002.

Longland, A. C., Cairns, A. J. und Humphreys, M. O., (1999): Seasonal and diurnal changes in fructan concentration in Lolium perenne: Implications for the grazing management of equines pre-disposed to laminitis.

16. Proc. Equine Nutr. Physiol. Soc., 258-259.

Longland, A. C. und Cairns A. J., (2000): Fructans and their implications in the aetiology of laminitis. Dodson \& Horell Ltd., 3rd International Conference on Feeding Horses, 52-55.

Mackenzie, D. J. und Wylam, C. B., (1957): Analytical studies on the carbohydrates of grasses and clovers. VIII. Changes in carbohydrate composition during the growth of perennial rye-grass. J. Sci. Food Agric., 8, 38-45.

Martens, D. S. und Greef, J.M., (2003): Sweetgrass - Der Anbau von zuckerreicher Deutschen Weidelgras (Q 12).

Mitteilungen Arbeitsgemeinschaft Grünland und Futterbau 2003, Band 5, 229-230

47. Jahrestagung, 28. bis 30. August 2003 in Braunschweig.

Matthes, K., (1986): Beziehungen zwischen Sortencharakter und den Gehalten wasserlöslicher Kohlenhydrate sowie verschiedener Strukturbestandteile bei der Art Lolium perenne L.; Dissertation, Universität Hohenheim.

Mcgrath, D., (1988): Seasonal variation in the water-soluble carbohydrates of perennial and Italian ryegrass under cutting conditions.

Irish J. Agric. Res., 27, 131-139.

Meak, P., (2002): Biochemische Charakterisierung von Pflanzen unterschiedlicher Nutzungsintensität zur Ableitung von Parametern für die Ermittlung des energetischen Futterwertes. Dissertation Landwirtschaftlich-Gärtnerische Fakultät, Humboldt-Univ., Berlin. 
Meister, E. und Lehmann, J., (1984): Art- und Sortenunterschiede der wichtigsten Futterleguminosen und Gräser in bezug auf den Gehalt an Rohprotein, Rohfaser und leicht vergärbaren Kohlehydraten. Mitteilungen für die Schweizerische Landwirtschaft, 32, Nr. 11, 210-224.

Menke, K.H., (1984): Bedeutung der Grundfutterqualität für die Futteraufnahme und Grundfutterverdrängung. Vortrag, Hülsenberger Gespräche, Travemünde.

Meyer, H. und Coenen, M., (2002): Pferdefütterung. 4. Aufl., Verlag Paul Parey, Berlin.

Miller, L.A., Baker, D.H., Theodorou, M.K., Macrae, J.C., Humphreys, M.O. und Scollan, N.D., (2001): efficiency of nitrogen use in dairy cows grazing ryegrasses with different water soluble carbohydrate concentrations in gassland ecosystems. In: Grassland Ecosystems: An Outlook into the $21^{\text {st }}$ Century, Proceedings of the XIX International Grassland Congres (Eds J.A. Gomide and W.R.S. Mattos), Sao Paolo, Brazil.

Miller, L.A., Moorby, J.M., Davies, D.R., Humphreys, M.O., Scollan, N.D., Macrae, J.C. und Theodorou, M.K., (2001): Increased concentration of water-soluble carbohydrate in perennial ryegrass (Loluim perenne L.): milk production from latelactation cows. Grass and Forage Science, 56, 383-394.

Mitterer, C., Rattler, S., Lux-Endrich, A., Grimoldi, A. and Gebbing, T., (2002): Einfluss der Mykorrhizierung auf die Blattbildung und Kohlenhydratgehalte von Deutsch WeidelgrasPflanzen. 46. Jahrestag. AG Grünland und Futterbau 4, 260-263.

Morcuende, R., Kostadinova, S. Pérez, P., Martín del Molino, I. M., Martínez-Carrasco, R., (2004): Nitrate is a negative signal for fructan synthesis, and the fructosyltransferase-inducing trehalose inhibits nitrogen and carbon assimilation in excised barley leaves. New Phytologist, Volume 161, Number 3, March 2004, pp. 749-759 (11).

Murata, Y., (1969): Physiological responses to nitrogen in Plants.

In: Physiological aspects of crop yield. (Eds. J.D Eastin: F.A. Haskins, C.Y. Sullivan and C.H.M. van Bavel), Amer. Soc. Agron., Madison, Wisconsin, 235-259. 
Muschick, M., (1998): Fruktanbiosynthese in Gramineae: Identifikation, Reinigung und Charakterisierung von Fruktan: Fruktan Fruktosyltransferasen zum Aufbau von Phleinen und Graminanen. Dissertation Naturwiss. ETH Zürich, Nr. 12571.

Neff, R., (1991): Zum Problem der Futterbewertung bei sehr später Nutzung. Jahrestagung der Arbeitgemeinschaft für Grünland und Futterbau in der Gesellschaft für Pflanzenbauwissenschaften, Tagungsbericht, 13-21.

Nõmmsalu H., (1994): The nutritive value of fodder galega (Galega orientalis Lam.). In: H. Nõmmsalu (Hrsg.), Fodder Galega (Galega orientalis Lam.) Research in Estonia, the Estonia Research Institute of Agriculture, Saku, 1994, 25-31.

Ojima, K. und Isawa, T., (1968): The variation of carbohydrates in various species of grasses and legumes. Can. J. Bot., 46, 1507-1511.

Ombabi, A., Südekum, K.-H. und Taube, F., (2001): Untersuchungen am Primäraufwuchs zweier Weidelgräser zur Dynamik der Veränderungen im Futterwert und der Futteraufnahme durch Schafe. J. Anim. Physiol. Anim. Nutr. 85, 385-405.

Paul, Ch., Alex, M., und Sommer, M., (2006): Fruktan in Gräsern: Auslöser einer Stoffwechselerkrankung beim Pferd - Bedeutung für die Weidewirtschaft und Heubereitung. 47. Fachtagung des DLG-Ausschusses „Gräser, Klee und Zwischenfrüchte“ Züchtungsperspektiven und Saatgutproduktion bei Gräsern, Klee und Zwischenfrüchten Vorträge der Fachtagung vom 5 und 6. Dezember 2006 in Fulda, 85-92.

Pavis, N., Boucaud, J. und Prud'homme, M. P., (2001): Fructans and fructan-metabolizing enzymes in leaves of Lolium perenne.

New Phytol. 150 (2001), pp. 97-109.

Pavis, N., Chatterton, N. J., Harrison, P. A., Baumgartner, S., Praznik, W., Boucaud, J. und Prud'homme, M. P., (2001): Structure of fructans in roots and leaf tissues of Lolium perenne. New Phytol. 150 (2001), pp. 83-95. 
Peacock, J.M., (1975): Temperature and leaf growth in Lolium perenne. III. Factors effecting seasonal differences. J. appl. Ecol. 12, 685-697.

Pfingstner, H., (1993): Produktionskosten und Wettbewerb in der Rinderhaltung. Schriftenreihe der Bundesanstalt für Agrarwirtschaft Wien, Nr. 72, 160 S.

Pollock, C. J. und Cairns, A. J., (1991): Fructan metabolism in grasses and cereals. Annu. Rev. Plant Physiol. Plant Mol. Biol., 42, 77-101.

Pötsch, E.M. und Resch, R., (2005): Einfluss unterschiedlicher Bewirtschaftungsmaßnahmen auf den Nährstoffgehalt von Grünlandfutter. Bericht 32. Viehwirtschaftliche Fachtagung. HBLFA Raumberg-Gumpenstein, 13.-14.04.2005, 1-14.

Robowsky, K.D., Kaltofen, H. und Knabe, O., (1977): Yield formation and carbohydrate content of Lolium perenne L. depending on nitrogen fertilization and cutting height. Proc. XIII. Int. Grassld. Congr, Leibzig, Sect. 1, 98-103.

Robson, M.J., (1980): A physiologist's approach to raising the potential yield of grass crop through breeding. In: Opportunities for increasing Crop Yield. (Eds. R.G. Hurd, P.V. Biscoe und C. Dennis), Pitman Publ. Ltd., London, 33-49.

Robson, M.J., (1981): Respiratory efflux in relation to Temperature of simulated swards of perennial ryegrass with contrasting soluble carbohydrate contents. Ann. Bot. 48, 269-273.

Rooke, J.A., Lee, N.H. und Armstrong, D.G., (1987): The effects of intraruminal infusions of urea, casein, glucose syrup and a mixture of casein and glucose syrup on nitrogen digestion in the rumen of cattle receiving grass-silage diets. British Journal of Nutrition 57; 89-98.

Schubiger, F. X., Bosshard, H.-R. und Lehmann, J., (1998): Nicht-strukturbildende Kohlenhydrate im Wiesenfutter. Agrarforschung 5, Nr. 2, 65-68.

Smith, D., (1968a): Carbohydrates in grasses. IV. Influence of temperature on the sugar and fructosan composition of Timothy plant parts at anthesis.

Crop Science, 8, 331-334. 
Smouter, H. und Simpson, R. J., (1989): Occurence of fructans in the Gramineae (Poaceae). New Phytol., 111, 359-368.

Smouter, H., Simpson, R. J. und Pearce, G. R., (1995): Water-soluble carbohydrates and in vitro digestibility of annual ryegrass (Lolium rigidum Gaudin) sown at varying densities. Aust. J. Agric. Res., 46, 611-625.

Sölter, U., (2007): Ackerfutterleguminosen und leguminosenbasierte Weiden in extensiver Bewirtschaftung für die Wiederkäuerfütterung, Dissertation, Universität Göttingen.

Spatz, G. und Fricke, T., (1992): Quantitatives und qualitatives Ertragsverhalten unterschiedlich spät geschnittener Grünlandbestände. Agribiol. Res. 45, 121-130.

Stauss, R., Bleiholder, H., Van Den Boom, T., Buhr, L., Hack, H., Hess, M., Klose, R., Meier, U. und Weber, E., (1994): Einheitliche Codierung der phänologischen Entwicklungsstadien mono- und dikotyler Pflanzen. Erweiterte BBCH-Skala: Allgemein. Ciba-Geigy AG, Basel, $58 \mathrm{~S}$.

Südekum, K.-H., Taube, F. und Wörner, M., (1990): Einfluß der botanischen Zusammensetzung (Weißkleeanteil) auf die Veränderung des energetischen Futterwertes im Zuwachsverlauf von Dauergrünlandbeständen. Kongreßband 102. VDLUFA -Schriftenreihe, 32, 469-474.

Taube, F., Wörner, M. und Südekum, K.-H., (1990): Veränderungen ausgewählter Qualitätsparameter im Zuwachsverlauf von Dauergrünlandbeständen in Abhängigkeit von der botanischen Zusammensetzung (Weißkleeanteil). Kongreßband 102. VDLUFA-Schriftenreihe, 32, 463-468, Berlin.

Techow, A., Herrmann, A., Taube, F., (2007): Leistungspotential von di- und tetraploiden Sorten des Deutschen Weidelgras - Was ist aus der Beschreibenden Sortenliste abzuleiten? Mitteilungen Arbeitsgemeinschaft Grünland und Futterbau 2007, Band 8, 60-63 51. Jahrestagung, 28. bis 30. August 2007 in Göttingen. 
Theimer, M., (2000): Literaturrecherche zur Winterhärte des Deutschen Weidelgrases.

Diplomarbeit 29. Oktober 2000.

http://www.lfl-neu.bayern.de/ipz/gruenland/16172/diplom2.pdf (14.03.07).

Trockels, F., (2004): Sortenzüchtung, Grundfutterqualität und Grundfutterleistung des Grünlandes (Teil 2: Qualität und Resistenzen). Deutsche Saatveredelung AG, Lippstadt, Veranstaltungsprogramm 31. August 2004 in Overath - Brombach.

http://www.versuchsgut-merklingsen.de/Publikationen/TagungenGruenlandbewirt-schaftung 2004/Much31.08.04/VortragTrockelsTeil2.pdf (28.06.07).

Ullrich, E., und Golze, M., (2004): Liebe und Leid gehen durch den Magen - Hufrehe. Infodienst 08/2004 Tierische Erzeugung.

http://www.landwirtschaft.sachsen.de/lfl/publikationen/download/787_1.pdf (5.7.2007).

Ulvund, M. J. und Pestalozzi, M., (1990): The possible significance of fructan in pasture grass for the development of ovine white-liverdisease (OWLD).

Acta vet. scand., 31, Nr. 3, 373-375.

VDLUFA Methodenbuch Band III (1997): Die chemische Untersuchung von Futtermitteln.

Verband Deutscher Landwirtschaftlicher Untersuchungs- Und Forschungsanstalten (Hrsg.)

VDLUFA-Verlag, Darmstadt.

Vermorel, M., (1989): Energy: The feed unit system. In Ruminant Nutrition. Recommended Allowances and Feed Tables. Ed. R. Jarrige, Inra, John Libbey Eurotext. ParisLondonpRome, 23-32.

Vervuert, I., Coenen, M., Dahlhoff, S., Sommer, W., (2004): Fruktane im Gras richtig einschätzen. Pferde Zucht und Haltung 12: 134-138.

Virkajärvi, P. und Varis, E., (1991): The effect of cutting times on goat's rue (Galega orientalis Lam.) leys. Journal of Agricultural Science in Finland, 63, 391-402.

Voigtländer, G. und Jacob, H., (1987): Lehrbuch: Grünlandwirtschaft und Futterbau. Verlag Ulmer, Stuttgart, 39-113. 
von Brostel, U. und Gräßler, J., (2003): Untersuchungen zur Kennzeichnung der Fructangehalte verschiedener Gräserarten. Mitteilungen Arbeitsgemeinschaft Grünland und Futterbau 2003, Band 5, 209-212, 47. Jahrestagung, 28. bis 30. August 2003 in Braunschweig.

Wilman, D. und Altimimi, M. A. K., (1982): "The diggestibility and chemical composition of plant parts in italian and perennial ryegrass during primary growth".

J. Sci. Food Agric., 33, 595-60.

Wolf, R. und Briemle, G., (1989): Landwirtschaftliche Verwertungsmöglichkeiten von Pflanzenaufwüchsen aus extensiviertem Grünland und aus der Biotoppflege. Das Wirtschaftseigene Futter, 35, 108-125. 


\section{Anhang}

Tab. A1: Der prozentuale Anteil des 1. Schnittes am Jahres-TM-Ertrag auf beiden Versuchsstandorten (BS und HS), bei beiden Schnittzeitpunktsvarianten (FSV und SSV) und im beiden Hauptsversuchsjahren (2006 und 2007).

\begin{tabular}{|c|c|c|c|c|c|c|c|c|}
\hline \multirow{2}{*}{ Sorten } & \multicolumn{4}{|c|}{ BS } & \multicolumn{3}{c|}{ HS } \\
\cline { 2 - 9 } & \multicolumn{2}{|c|}{ FSV } & \multicolumn{2}{c|}{ SSV } & \multicolumn{2}{c|}{ FSV } & \multicolumn{2}{c|}{ SSV } \\
\cline { 2 - 9 } & 2006 & 2007 & 2006 & 2007 & 2006 & 2007 & 2006 & 2007 \\
\hline Licarta & 48 & 35 & 61 & 54 & 45 & 45 & 45 & 48 \\
\hline Abersilo & 49 & 41 & 59 & 52 & 43 & 36 & 45 & 47 \\
\hline EGB1 & 43 & 35 & 59 & 51 & 42 & 39 & 45 & 45 \\
\hline Respect & 45 & 34 & 57 & 53 & 41 & 37 & 42 & 46 \\
\hline Betty & 47 & 33 & 59 & 45 & 44 & 42 & 46 & 46 \\
\hline Aberdart & 45 & 33 & 57 & 50 & 41 & 38 & 43 & 45 \\
\hline Rodrigo & 42 & 37 & 58 & 49 & 39 & 35 & 42 & 44 \\
\hline EGB2 & 37 & 25 & 52 & 44 & 34 & 38 & 40 & 40 \\
\hline Aubisque & 47 & 33 & 58 & 51 & 39 & 37 & 43 & 46 \\
\hline Sponsor & 38 & 27 & 53 & 46 & 35 & 37 & 40 & 41 \\
\hline AberAvon & 40 & 30 & 54 & 44 & 37 & 37 & 41 & 41 \\
\hline EGB3 & 35 & 26 & 48 & 42 & 37 & 35 & 40 & 40 \\
\hline EGB4 & 36 & 23 & 52 & 44 & 33 & 34 & 39 & 38 \\
\hline EGB5 & 31 & 24 & 50 & 36 & 31 & 30 & 39 & 35 \\
\hline Bocage & 37 & 26 & 53 & 41 & 35 & 32 & 39 & 37 \\
\hline Liherold & 54 & 31 & 61 & 50 & 45 & 41 & 48 & 45 \\
\hline Litornado & 49 & 27 & 61 & 49 & 40 & 41 & 48 & 42 \\
\hline Lifema & 52 & 33 & 63 & 51 & 45 & 46 & 48 & 49 \\
\hline Lidaglo & 42 & 25 & 51 & 43 & 35 & 33 & 40 & 38 \\
\hline Lischka & 54 & 36 & 62 & 58 & 39 & 45 & 46 & 43 \\
\hline
\end{tabular}


Tab. A2: Verteilung des Fruktangehaltes (\%) nach dem Polymerisationsgrad für Sorte Respect in Gemischprobe, Stängel und Blätter im 2HVJ (2007) in allen Erntenterminen.

\begin{tabular}{|c|c|c|c|c|c|c|c|c|c|c|c|}
\hline \multicolumn{12}{|c|}{ Gemischprobe } \\
\hline Gfn & 1. Ernte & 2. Ernte & 3. Ernte & 4. Ernte & 5. Ernte & 6. Ernte & 7. Ernte & 8. Ernte & 9. Ernte & 10. Ernte & 11. Ernte \\
\hline 3-12 & 65,27 & 53,47 & 50,85 & 51,59 & 45,78 & 46,52 & 40,76 & 38,60 & 44,24 & 33,92 & 34,58 \\
\hline $13-22$ & 19,10 & 26,03 & 24,64 & 21,89 & 24,66 & 22,39 & 28,30 & 26,42 & 18,90 & 21,61 & 27,80 \\
\hline $23-32$ & 9,91 & 10,40 & 10,93 & 13,51 & 15,03 & 14,67 & 12,39 & 13,45 & 14,25 & 17,01 & 15,89 \\
\hline $33-42$ & 4,48 & 5,92 & 6,90 & 6,58 & 7,90 & 7,76 & 9,18 & 11,46 & 11,72 & 13,73 & 13,39 \\
\hline $43-52$ & 0,65 & 3,74 & 3,58 & 5,43 & 5,89 & 6,59 & 7,74 & 8,52 & 8,83 & 11,34 & 8,34 \\
\hline$>52$ & 0,58 & 0,45 & 3,10 & 0,99 & 0,75 & 2,08 & 1,63 & 1,54 & 2,05 & 2,39 & \\
\hline \multicolumn{12}{|c|}{ Stängel } \\
\hline \multicolumn{12}{|l|}{ Gfn } \\
\hline $3-12$ & & & & 53,32 & 48,96 & 47,56 & 44,24 & 38,40 & 41,66 & 30,82 & 26,19 \\
\hline $13-22$ & & & & 16,88 & 20,41 & 21,80 & 21,78 & 24,13 & 17,35 & 21,27 & 27,63 \\
\hline $23-32$ & & & & 13,06 & 13,38 & 14,73 & 13,33 & 14,26 & 14,23 & 16,91 & 17,36 \\
\hline $33-42$ & & & & 8,32 & 9,44 & 7,97 & 9,78 & 11,89 & 13,60 & 15,33 & 15,43 \\
\hline $43-52$ & & & & 6,85 & 6,26 & 6,76 & 8,66 & 9,24 & 10,45 & 12,28 & 11,90 \\
\hline$>52$ & & & & 1,57 & 1,56 & 1,18 & 2,20 & 2,08 & 2,72 & 3,38 & 1,49 \\
\hline \multicolumn{12}{|c|}{ Blätter } \\
\hline \multicolumn{12}{|l|}{ Gfn } \\
\hline 3-12 & & & & 46,07 & 40,42 & 45,51 & 34,30 & 34,17 & 34,01 & 33,11 & 51,83 \\
\hline $13-22$ & & & & 23,89 & 26,19 & 29,07 & 29,83 & 31,46 & 26,54 & 27,61 & 23,14 \\
\hline 23-32 & & & & 13,87 & 15,95 & 12,65 & 12,77 & 13,33 & 15,98 & 17,18 & 13,37 \\
\hline $33-42$ & & & & 9,09 & 9,87 & 6,47 & 11,51 & 10,89 & 12,16 & 11,00 & 6,25 \\
\hline $43-52$ & & & & 5,86 & 6,37 & 5,41 & 9,79 & 7,22 & 8,33 & 8,14 & 0,66 \\
\hline$>52$ & & & & 1,21 & 1,20 & 0,90 & 1,80 & 2,93 & 2,98 & 2,96 & 4,75 \\
\hline
\end{tabular}

Tab. A3: Verteilung des Fruktangehaltes (\%) nach dem Polymerisationsgrad für Sorte Licarta in Gemischprobe, Stängel und Blätter im 2HVJ (2007) in allen Erntenterminen.

\begin{tabular}{|c|c|c|c|c|c|c|c|c|c|c|c|}
\hline \multicolumn{12}{|c|}{ Gemischprobe } \\
\hline Gfn & 1. Ernte & 2. Ernte & 3. Ernte & 4. Ernte & 5. Ernte & 6. Ernte & 7. Ernte & 8. Ernte & 9. Ernte & 10. Ernte & 11. Ernte \\
\hline $3-12$ & 67,32 & 55,90 & 60,36 & 45,99 & 41,71 & 44,63 & 40,09 & 42,56 & 40,61 & 34,31 & 39,05 \\
\hline $13-22$ & 19,37 & 25,19 & 16,61 & 23,55 & 23,96 & 30,17 & 27,69 & 19,69 & 21,15 & 20,84 & 22,80 \\
\hline $23-32$ & 7,65 & 10,01 & 10,31 & 15,06 & 16,45 & 12,66 & 12,87 & 14,39 & 13,85 & 16,99 & 15,48 \\
\hline $33-42$ & 3,42 & 5,39 & 7,08 & 8,73 & 9,07 & 6,55 & 9,31 & 11,88 & 11,08 & 13,73 & 11,59 \\
\hline $43-52$ & 0,17 & 3,24 & 4,26 & 5,61 & 7,09 & 5,31 & 7,98 & 8,55 & 10,08 & 11,20 & 9,14 \\
\hline$>52$ & 1,00 & 0,27 & 1,39 & 1,06 & 1,72 & 0,69 & 2,05 & 2,94 & 3,22 & 2,93 & 1,93 \\
\hline \multicolumn{12}{|c|}{ Stängel } \\
\hline \multicolumn{12}{|l|}{ Gfn } \\
\hline $3-12$ & & & & & & 42,41 & 39,31 & 39,69 & 35,84 & 30,25 & 29,48 \\
\hline $13-22$ & & & & & & 24,72 & 26,08 & 19,80 & 23,93 & 20,70 & 24,78 \\
\hline $23-32$ & & & & & & 13,09 & 13,45 & 14,19 & 12,44 & 17,43 & 15,21 \\
\hline $33-42$ & & & & & & 9,79 & 11,32 & 13,45 & 13,07 & 16,29 & 15,46 \\
\hline 43-52 & & & & & & 7,90 & 7,81 & 9,87 & 11,71 & 12,51 & 11,89 \\
\hline$>52$ & & & & & & 2,10 & 2,03 & 3,02 & 3,01 & 2,81 & 3,18 \\
\hline \multicolumn{12}{|c|}{ Blätter } \\
\hline \multicolumn{12}{|l|}{ Gfn } \\
\hline $3-12$ & & & & & & 39,39 & 40,71 & 32,12 & 37,07 & 35,15 & 50,64 \\
\hline 13-22 & & & & & & 24,21 & 22,71 & 32,82 & 26,43 & 26,76 & 27,55 \\
\hline $23-32$ & & & & & & 15,84 & 14,23 & 13,28 & 14,62 & 15,36 & 12,81 \\
\hline $33-42$ & & & & & & 9,83 & 12,27 & 11,88 & 11,58 & 11,71 & 7,62 \\
\hline $43-52$ & & & & & & 8,67 & 8,33 & 7,84 & 8,18 & 9,12 & 1,38 \\
\hline$>52$ & & & & & & 2,05 & 1,75 & 2,06 & 2,12 & 1,89 & \\
\hline
\end{tabular}


Tab. A4: Verteilung des Fruktangehaltes (\%) nach dem Polymerisationsgrad für Sorte EGB1 in Gemischprobe, Stängel und Blätter im 2HVJ (2007) in allen Erntenterminen.

\begin{tabular}{|c|c|c|c|c|c|c|c|c|c|c|c|}
\hline \multicolumn{12}{|c|}{ Gemischprobe } \\
\hline Gfn & 1. Ernte & 2. Ernte & 3. Ernte & 4. Ernte & 5. Ernte & 6. Ernte & 7. Ernte & 8. Ernte & 9. Ernte & 10. Ernte & 11. Ernte \\
\hline $3-12$ & 70,33 & 55,49 & 55,43 & 48,20 & 45,67 & 51,38 & \multirow{6}{*}{ 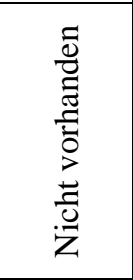 } & 42,59 & 41,18 & 38,53 & 38,93 \\
\hline $13-22$ & 16,99 & 24,65 & 21,55 & 24,07 & 24,25 & 22,11 & & 20,95 & 19,91 & 21,55 & 26,87 \\
\hline 23-32 & 7,60 & 9,66 & 11,48 & 14,63 & 15,13 & 13,34 & & 14,48 & 15,87 & 16,48 & 13,84 \\
\hline 33-42 & 3,35 & 5,24 & 7,23 & 6,99 & 7,53 & 6,08 & & 10,48 & 11,46 & 11,98 & 9,74 \\
\hline $43-52$ & 1,50 & 3,41 & 3,47 & 5,57 & 6,63 & 5,94 & & 8,58 & 9,25 & 9,39 & 7,55 \\
\hline$>52$ & 0,23 & 1,56 & 0,84 & 0,54 & 0,78 & 1,16 & & 2,91 & 2,34 & 2,07 & 3,07 \\
\hline \multicolumn{12}{|c|}{ Stängel } \\
\hline \multicolumn{12}{|l|}{ Gfn } \\
\hline $3-12$ & & & & & & 46,47 & 54,32 & 43,95 & 41,42 & 35,29 & 34,22 \\
\hline $13-22$ & & & & & & 24,90 & 23,81 & 20,50 & 19,80 & 16,78 & 24,11 \\
\hline 23-32 & & & & & & 11,95 & 9,84 & 14,34 & 15,30 & 18,10 & 15,49 \\
\hline 33-42 & & & & & & 8,71 & 5,77 & 9,81 & 11,21 & 14,82 & 12,99 \\
\hline $43-52$ & & & & & & 6,71 & 5,23 & 9,32 & 10,07 & 11,96 & 10,84 \\
\hline$>52$ & & & & & & 1,24 & 1,02 & 2,08 & 2,20 & 3,05 & 2,35 \\
\hline \multicolumn{12}{|c|}{ Blätter } \\
\hline \multicolumn{12}{|l|}{ Gfn } \\
\hline $3-12$ & & & & & & 45,73 & 38,76 & 39,64 & 38,09 & 44,17 & 41,96 \\
\hline $13-22$ & & & & & & 23,81 & 29,50 & 25,59 & 28,09 & 20,14 & 25,10 \\
\hline $23-32$ & & & & & & 14,46 & 11,81 & 14,77 & 14,36 & 15,52 & 16,06 \\
\hline $33-42$ & & & & & & 7,65 & 10,17 & 11,27 & 10,35 & 9,66 & 7,92 \\
\hline $43-52$ & & & & & & 7,11 & 8,48 & 7,45 & 7,65 & 8,06 & 8,95 \\
\hline$>52$ & & & & & & 1,25 & 1,28 & 1,29 & 1,46 & 2,46 & \\
\hline
\end{tabular}

Tab. A5: Verteilung des Fruktangehaltes (\%) nach dem Polymerisationsgrad für Sorte Rodrigo in Gemischprobe, Stängel und Blätter im 2HVJ (2007) in allen Erntenterminen.

\begin{tabular}{|c|c|c|c|c|c|c|c|c|c|c|c|}
\hline \multicolumn{12}{|c|}{ Gemischprobe } \\
\hline Gfn & 1. Ernte & 2. Ernte & 3. Ernte & 4. Ernte & 5. Ernte & 6. Ernte & 7. Ernte & 8. Ernte & 9. Ernte & 10. Ernte & 11. Ernte \\
\hline $3-12$ & 67,27 & 51,42 & 55,57 & 49,04 & 42,06 & 41,75 & 41,77 & 47,76 & 42,33 & 42,85 & 40,08 \\
\hline $13-22$ & 18,47 & 23,16 & 18,55 & 22,71 & 24,71 & 24,60 & 27,79 & 18,66 & 17,04 & 17,72 & 22,14 \\
\hline $23-32$ & 9,24 & 11,86 & 13,65 & 14,49 & 15,47 & 15,66 & 13,36 & 13,78 & 15,69 & 15,80 & 15,93 \\
\hline $33-42$ & 3,85 & 7,04 & 7,34 & 7,05 & 8,47 & 8,86 & 8,89 & 9,08 & 11,83 & 10,99 & 10,90 \\
\hline $43-52$ & 0,33 & 5,43 & 4,46 & 5,86 & 7,99 & 7,41 & 6,42 & 8,46 & 10,13 & 9,42 & 9,29 \\
\hline$>52$ & 0,83 & 1,09 & 0,43 & 0,85 & 1,30 & 1,72 & 1,77 & 2,26 & 2,98 & 3,23 & 1,66 \\
\hline \multicolumn{12}{|c|}{ Stängel } \\
\hline \multicolumn{12}{|l|}{ Gfn } \\
\hline $3-12$ & & & & & & 43,43 & 47,21 & 43,95 & 38,04 & 32,75 & 32,48 \\
\hline $13-22$ & & & & & & 20,90 & 18,30 & 17,51 & 17,96 & 23,27 & 24,26 \\
\hline 23-32 & & & & & & 16,10 & 13,63 & 14,66 & 16,98 & 15,34 & 16,25 \\
\hline $33-42$ & & & & & & 9,73 & 9,67 & 11,98 & 12,96 & 14,81 & 13,25 \\
\hline 43-52 & & & & & & 7,98 & 8,93 & 9,50 & 11,17 & 11,10 & 11,06 \\
\hline$>52$ & & & & & & 1,86 & 2,26 & 2,40 & 2,90 & 2,73 & 2,70 \\
\hline \multicolumn{12}{|c|}{ Blätter } \\
\hline \multicolumn{12}{|l|}{ Gfn } \\
\hline 3-12 & & & & & & 40,97 & 39,67 & 40,29 & 36,21 & 39,37 & 45,38 \\
\hline $13-22$ & & & & & & 21,03 & 25,72 & 19,68 & 23,08 & 29,13 & 27,73 \\
\hline $23-32$ & & & & & & 18,11 & 12,73 & 15,61 & 17,05 & 17,29 & 14,31 \\
\hline $33-42$ & & & & & & 8,95 & 10,71 & 12,69 & 11,31 & 9,11 & 7,50 \\
\hline $43-52$ & & & & & & 8,53 & 8,75 & 9,37 & 9,44 & 5,10 & 3,89 \\
\hline$>52$ & & & & & & 2,42 & 2,41 & 2,36 & 2,91 & & 1,19 \\
\hline
\end{tabular}


Tab. A6: Verteilung des Fruktangehaltes (\%) nach dem Polymerisationsgrad für Sorte AberAvon in Gemischprobe, Stängel und Blätter im 2HVJ (2007) in allen Erntenterminen.

\begin{tabular}{|c|c|c|c|c|c|c|c|c|c|c|c|}
\hline \multicolumn{12}{|c|}{ Gemischprobe } \\
\hline Gfn & 1. Ernte & 2. Ernte & 3. Ernte & 4. Ernte & 5. Ernte & 6. Ernte & 7. Ernte & 8. Ernte & 9. Ernte & 10. Ernte & 11. Ernte \\
\hline $3-12$ & 74,85 & 61,94 & 57,19 & 51,04 & 44,14 & 45,03 & 39,18 & 48,72 & 40,07 & 36,55 & 47,69 \\
\hline $13-22$ & 13,60 & 20,69 & 18,74 & 21,61 & 23,05 & 22,20 & 26,87 & 18,21 & 19,38 & 22,23 & 20,05 \\
\hline $23-32$ & 7,70 & 9,61 & 12,19 & 13,78 & 16,26 & 15,67 & 13,79 & 14,84 & 17,06 & 16,99 & 13,95 \\
\hline $33-42$ & 2,85 & 4,08 & 7,33 & 7,20 & 7,77 & 8,03 & 10,18 & 8,95 & 11,19 & 12,98 & 9,30 \\
\hline $43-52$ & 0,84 & 2,58 & 3,85 & 5,36 & 7,37 & 7,28 & 7,83 & 7,15 & 9,87 & 10,44 & 7,35 \\
\hline$>52$ & 0,16 & 1,09 & 0,70 & 1,01 & 1,40 & 1,80 & 2,16 & 2,12 & 2,43 & 0,80 & 1,66 \\
\hline \multicolumn{12}{|c|}{ Stängel } \\
\hline \multicolumn{12}{|l|}{ Gfn } \\
\hline 3-12 & & & & & & 61,42 & 54,20 & 51,71 & 46,35 & 34,74 & 36,79 \\
\hline $13-22$ & & & & & & 16,79 & 19,26 & 14,27 & 12,75 & 23,28 & 21,77 \\
\hline $23-32$ & & & & & & 9,34 & 9,84 & 14,31 & 16,86 & 15,17 & 17,13 \\
\hline $33-42$ & & & & & & 5,72 & 8,41 & 9,67 & 11,42 & 14,54 & 11,82 \\
\hline $43-52$ & & & & & & 5,09 & 6,06 & 7,59 & 9,81 & 10,22 & 10,21 \\
\hline$>52$ & & & & & & 1,64 & 2,22 & 2,45 & 2,80 & 2,05 & 2,28 \\
\hline \multicolumn{12}{|l|}{ Blätter } \\
\hline \multicolumn{12}{|l|}{ Gfn } \\
\hline $3-12$ & & & & & & 44,27 & 40,44 & 43,50 & 40,62 & 20,86 & 45,68 \\
\hline $13-22$ & & & & & & 23,54 & 21,39 & 19,65 & 20,23 & 31,06 & 24,74 \\
\hline $23-32$ & & & & & & 15,27 & 14,62 & 15,22 & 15,67 & 23,38 & 13,11 \\
\hline $33-42$ & & & & & & 8,05 & 11,58 & 11,13 & 11,19 & 13,66 & 7,69 \\
\hline $43-52$ & & & & & & 7,27 & 9,09 & 8,57 & 9,96 & 10,32 & 6,11 \\
\hline$>52$ & & & & & & 1,59 & 2,89 & 1,93 & 2,34 & 0,70 & 2,67 \\
\hline
\end{tabular}




\section{Danksagung}

Mein herzlicher Dank für die freundliche Unterstützung gilt all jenen, die zum Gelingen der vorliegenden Arbeit beigetragen haben:

- Herrn Prof. Dr. J. M. Greef für die Überlassung des Themas, seine liebevolle Betreuung, die vielfältigen konstruktiven Hinweise und die gewährte Unterstützung bei der Fertigstellung der Arbeit.

- Herrn Prof. Dr. J. Isselstein für die Übernahme der Begutachtung meiner Arbeit.

- Herrn Prof. Dr. Hansjörg Abel danke ich für die bereitwillige Übernahme des Koreferates.

- Herrn Dr. U. Feuerstein und seinen Mitarbeiterinnen und Mitarbeitern von Euro Grass Breeding Hof Steimke für die Durchführung dieser Arbeit auf Versuchsstandort Hof Steimke.

- Frau Dr. Ulrike Sölter und Frau Dr. Maren Langhof für das Korrekturlesen dieser Arbeit.

- Herrn D. D. Strauß möchte ich für die Durchführung der statistischen Analyse mit SAS® danken.

Ein herzlicher Dank gilt allen Mitarbeiterinnen und Mitarbeitern unseres Institutes für die große Hilfsbereitschaft auf dem Feld, den Laboranalysen und Freundlichkeit. Besonders danke ich Melanie Schütt für ihre freundliche Unterstützung bei der Arbeit auf dem Feld und bei der Bearbeitung der Proben.

Ein herzlicher Dank gilt Al-Baath Universität in Syrien für großzügiges Stipendium.

Meiner Frau für ihre Geduld und das Mehr an Kinderziehung und Haushalt in der Zeit des Schreibens herzlich danken.

Von ganzem Herzen danke ich auch meinen Eltern.

Ebenso sei allen denen ein Dankeschön ausgesprochen, die nicht namentlich Erwähnung fanden, aber zum Gelingen dieser Arbeit beigetragen haben. 


\section{Lebenslauf}

Name

Vorname

Geburtsdatum

Geburtsort

Familienstand

Ausbildung

1981 - 1994

1994 - 1999

$1999-2000$

2002

$2002-2003$

$2004-2005$

Seit 2005

\section{Berufstätigkeit}

1999 - 2002

Seit 2005
Soufan

Walid

04.08.1975

Talbise- Syrien

Verheiratet, zwei Kinder

Grundschule und Oberschule in Homs - Syrien

Abschluss: Abitur

Fakultät für Agrarwissenschaften der Universität Al-Baath in Syrien Abschluss: Agraringenieur

Hochschuldiplom an der Universität Aleppo in Syrien

Promotionsstipendiat von Al-Baath Universität

Deutsche Sprachkurs

Anerkennung von Studienabschlüssen für die Zulassung zur Promotionsprüfung an Fakultät für Agrarwissenschaften der Georg-August-Universität zu Göttingen

Promotionsstudent an der Fakultät für Agrarwissenschaften der

Georg-August-Universität zu Göttingen

Wissenschaftlicher Mitarbeiter an der Fakultät für Agrarwissenschaften der

AL-Baath Universität

Wissenschaftlicher Mitarbeiter

Institut für Pflanzenbau und Grünlandwirtschaft der FAL in

Braunschweig (Fachgebiet „, Futterbau und Grünlandwirtschaft“ 